\section{Oral Session: Solid Tumors}

\section{O418}

\section{Combined Anti-Inflammatory and Angiostatic Treatment of Hormone Refractory Prostate Cancer}

\section{Reichle A. ${ }^{1}$, Rogenhofer $\mathrm{S}^{2}$, Wieland $W^{2}{ }^{2}$, Andreesen . $^{1}$ Department of Hematology and Oncology, Department of Urology, University Regensburg, Germany}

Purpose: Peroxisome proliferator-activated receptor gamma (PPARg) and cyclooxygenase-2 (COX-2) are frequently over-expressed in both prostate cancer cells and in the adjacent stroma cells, and could be targets for therapy of hormone refractory prostate cancer (HRPC). Methods: The current study for symptomatic patients with HRPC was designed to evaluate the efficacy and toxicity of repetitively administered low-dose capecitabine (twice daily $\mathrm{g} / \mathrm{m}^{2}$, day 14 to 28 , every 3 weeks) combined with an anti-inflammatory/ angiostatic treatment including rofecoxib (selective COX-2 inhibitor) $25 \mathrm{mg}$ po day $1+$, pioglitazone PPARg agonist, $60 \mathrm{mg}$ po day $1+$, and dexamethasone $1 \mathrm{mg}$ daily, day 14 to 28 , every 3 weeks during continuation of hormone ablation. Results: Eighteen men (median age 68 years), 10 of whom had received previous radiation and/ or chemotherapy (56\%), were enrolled onto study protocol. Using clinical response guidelines set forth by the Prostate Specific Antigen Working Group, 33\% of patients were found to have a $>$ or $=80 \%$ reduction of PSA, $11 \%$ of patients were found to have a reduction $<$ $80 \%$ and $>50 \%, 38 \%$ were found to have a $<50 \%$ decrease in PSA, and $17 \%$ experienced disease progression while receiving treatment. Up to date study medication was discontinued due to PSA progression in 4 patients after 3 to 6 months, 14 patients are still on treatment (median 6 months, range 2 to 9 months +). Two of 3 patients with measurable disease achieved partial remission. In $33 \%$ of patients Karnofsky performance status improved. 56\% of patients could reduce analgetic medication due to positive pain response Morphin medication could be terminated in two patients. Major toxicity (grade 3) was observed in one patient (edema). Conclusions: In contrast to the recently reported low efficacy of standard dose capecitabine in HRPC, the present all-oral therapy approach combining stroma cell- and tumor celltargeted therapy including repetitively administered low-dose capecitabine (metronomic chemotherapy) shows for the first time efficacy and low toxicity, which compares to standard therapy with docetaxel iv concerning PSA response and improvement of clinical response parameters.

\section{O419}

\section{Thalidomide in Refractory Germ-Cell Tumors}

Rick O. ${ }^{1}$, Beyer.J. ${ }^{2}$, Braun T. ${ }^{1}$, Marquart U. ${ }^{1}$, Siegert $W .{ }^{1}$

${ }^{1}$ Medizinische Klinik II m.S. Onkologie/Hämatologie,

Universitätsklinikum Charité, Campus Mitte, Humboldt Universität,

Berlin, Germany, ${ }^{2}$ Klinik für Innere Medizin m.S. Hämatologie/

Onkologie, Universitätsklinikum Marburg, Germany

Purpose: To assess the activity of thalidomide in patients (pts.) with germcell tumors (GCT) and progressive inoperable disease after salvage chemotherapy. Patients and Methods: Between April 2002 and January 2003, 15 pts were included in a phase I/II study and treated with escalated daily doses of 200 to $600 \mathrm{mg}$ of thalidomide. All pts had relapsed or progressed after salvage treatment and were considered inoperable. Pts has received a median of six (range, 4 to 12) cisplatin-based treatment cycles. Before study treatment 13/15 (87\%) pts had received high-dose chemotherapy (HDCT), 3/15 pts (20\%) were considered refractory and 5/15 (33\%) pts absolute refractory to cisplatin. Oral etoposide, gemcitabine plus oxaliplatin, combinations of paclitaxel, ifosfamide and cisplatin or other palliative treatments had been tried in $8 / 15(53 \%)$ pts prior to thalidomide administration. Results: Overall, 5/15 (33\%) pts achieved a marker-positive partial remission $(33 \%)$ and one additional patient achieved disease stabilization. The median duration of remission was 3 months (range of 2 to 12 months). Responses to thalidomide were independent from tumor mass, platinum-sensitivity or the degree of tumor marker elevations. However, responses were almost exclusively seen in pts with slowly increasing alphafetoprotein (AFP). Toxicity was mild, with lethargy and constipation in the majority of pts. Skin rash CTC grade II developed in two pts and neurotoxicity CTC grade II/III developed in four pts. One responding patient died suddenly from an unknown cause. Conclusions: Thalidomide shows singleagent activity in pts with heavily pretreated GCT, AFP elevations and slowly progressive disease.
O420

Low risk of secondary Leukemia following First-Line High-Dose Chemotherapy for Patients With Advanced Germ Cell Cancer

Wierecky J. ${ }^{1}$, Kollmannsberger C. ${ }^{1}$, Boehlke I. ${ }^{1}$, Metzner B. ${ }^{2}$, Kanz L. ${ }^{1}$, Hartmann J.T. ${ }^{1}$, Bokemeyer C. ${ }^{1}$

${ }^{1}$ Medizinische Klinik II, Universitätsklinikum Tübingen, Germany, ${ }^{2}$ Abteilung für Hämatologie und Onkologie, Klinikum Oldenburg, Germany

Purpose: We investigated the incidence of secondary leukemia (s-AML) in patients (pts) treated with first-line high-dose chemotherapy (HDCT) plus autologous peripheral stem cell transplantation (PBSCT) for advanced germ cell tumor (GCT). Methods: Three hundred twenty-three pts with median age of 30 (range, 15 - 47) from two consecutive prospective Phase-II studies of the German Testicular Cancer Study Group were analyzed. Of these 221 pts had received HD-VIP containing cisplatin, ifosfamide and etoposide and 102 pts were treated with Tax-HD-VIP containing cisplatin, ifosfamide, etoposide plus paclitaxel. All pts had received 3-4 HDCT cycles each supported by autologous PBSCT resulting in a median cumulative dose of etoposide of 4.9 $\mathrm{g} / \mathrm{m}^{2}$ (range, 2.2 to $9.4 \mathrm{~g} / \mathrm{m}^{2}$ ). Results: The median follow-up of pts alive at least one year after therapy and thus being at risk for the development of s-AML was 50 months (mo) (range, $12-128 \mathrm{mo}$ ). Two hundred thirty eight (74\%) pts were alive at a median follow-up of 46 mo (range, 3 to 118). One pat developed a secondary acute myeloid leukemia (s-AML) involving a chromosomal translocation $\mathrm{t}(11 ; 19)(\mathrm{q} 23 ; \mathrm{p} 13.3) 24$ mo after the start of chemotherapy and 16 mo after HD salvage chemotherapy (cumulative etoposide dose of $8 \mathrm{~g} / \mathrm{m}^{2}$ ) which he had subsequently received for relapsed diesease. This resulted in a cumulative incidence for secondary leukemia of $0.48 \%$ [95 \%-CI: 0-1.42]. Two additional pts with primary mediastinal GCT developed a myelodysplastic syndrome (MDS) 23 and 11 mo after chemotherapy considered to be non-therapy induced but biologically related to the GCT. No secondary solid tumors were observed. Conclusions: HDCT as first-line therapy for advanced testicular cancer was associated with an acceptably low risk of developing secondary malignancies including s-AML.

\section{O421 \\ Recombinant Adeno-associated Virus 2 Suicide Vectors for the Treatment of Human Sarcomas and Mesotheliomas}

Veldwijk M.R. ${ }^{1,2}$, Berlinghoff S. ${ }^{2}$, Laufs S. ${ }^{2}$, Herskind C. ${ }^{1}$, Zeller W.J. ${ }^{2}$, Wenz F. ${ }^{1}$, Fruehauf $S^{3}{ }^{3}$

${ }^{1}$ Department of Radiation Oncology, University of Heidelberg, Mannheim, Germany, ${ }^{2}$ German Cancer Research Center (E120), Heidelberg, Germany, ${ }^{3}$ Department of Internal Medicine V,

University of Heidelberg, Heidelberg, Germany

Although great efforts have been made to improve conventional therapy for sarcoma and malignant mesothelioma, the median survival time of these entities after appearance of clinical symptoms stays poor. Effective locoregional therapy using viral vectors that contain a suicide gene may be an alternative treatment strategy. For sarcoma, we previously reported the highest susceptibility for recombinant adeno-associated virus 2 (rAAV-2) vectors in human connective tissue sarcoma cells. Now we confirm our findings in five further human sarcoma cell lines: fibrosarcoma, Ewing's sarcoma, Askin's tumor, rhabdomyosarcoma and soft tissue sarcoma. Furthermore, we found that rAAV-2 also achieved both high transduction rates and GFP expression levels in three human mesothelioma cell lines. Among rAAV-2 constructs containing different promotors, after transduction, the vector with the elongation factor 1-alpha (EF1 $\alpha$ ) promotor showed the highest expression rates in both the sarcoma and mesothelioma cell lines. To ensure the use of constant vector particles number, all stocks were titrated using both the functional and our real-time PCR-based titration assay.

Several new thymidine kinase $(T K)$ gene-containing vectors under control of either the Cytomegalovirus or the EF1 $\alpha$ promoter were cloned and tested. A higher expression level of the transgene was observed in the sarcoma and mesothelioma lines when using the EF1 $\alpha$-suicide gene-containing vectors. For the sarcomas, we were able to show a complete eradication of all rAAV EF1 $\alpha-T K / e G F P$ (fusion gene) transduced tumor cells following exposure to ganciclovir $(2.5 \mu \mathrm{g} / \mathrm{ml})$ in vitro, while at this dose level $>90 \%$ of mock-transduced tumor cells survived. Using mesotheliomas, a nearly complete eradication (>2 log) of transduced and GCV-treated cells could be obtained using this vector. 
Xenotransplantation tumor models for both the human sarcoma (i.p., s.c.) and mesothelioma cell lines (i.p.) were established in NOD/SCID mice. In proofof-principle experiments, mice transplanted with rAAV-TK/eGFP-transduced and ganciclovir-exposed sarcoma or mesothelioma tumor cells survived $>5$ months while in the non-transduced group all mice had died approximately 1 month after inoculation. Currently, further animal experiments using this suicide system are ongoing.

The data shown here hold promise for further development of AAV-2-based suicide gene therapy of both soft tissue sarcoma and mesothelioma towards a future clinical application.

\section{$\mathrm{O} 422$}

Ifosfamide and Liposomal Daunorubicin (IDx) is a Well Tolerated and Active First Line Chemotherapy Regimen in Advanced Soft Tissue Sarcoma - Results of a Phase II Study

Siehl J.M., Keilholz U., Schmittel A., Hütter G., Deckert P.M., Szelényi H., Thiel E.

Medizinische Klinik III (Haematology, Oncology and Transfusion

Medicine), Charité, Campus Benjamin Franklin, Berlin, Germany

Purpose: Anthracycline/ifosfamide combination is the most effective chemotherapy in soft tissue sarcoma. To improve tolerability and potentially efficacy of this combination we combined high dose continuous infusion ifosfamide with liposomal daunorubicin (L-Dauno). Patients and Methods: In a single-arm phase II study 40 patients were enrolled with a median age of 57 years (19 to 78 years). Treatment regimen was ifosfamide $5 \mathrm{~g} / \mathrm{m}^{2}$ over $24 \mathrm{~h}$ and L-Dauno $100 \mathrm{mg} / \mathrm{m}^{2}$ every 4 weeks with G-CSF support. Initially, 5 patients were included after failure of a conventional doxorubicin/ifosfamide regimen, but none of them responded. Subsequently, 35 patients were treated first line. A total of 113 cycles (range 1 to 6) were given. Results: Eleven (31 $\%$ ) out of 35 anthracycline/ifosfamide naïve patients achieved a PR/CR, all after 2 treatment cycles, 6 patients $(17 \%)$ had stable disease and 13 patients (37 \%) progressed; 5 patients were not evaluable. PR with respect to histology was: 4/7 PNET, 1/6 leiomyosarcoma, 2/4 liposarcoma, 1/2 synovial sarcoma, 1/3 pleomorphic sarcoma, 1/1 malignant histiocytoma and 1/2 carcinosarcoma. Median time to progression was 6 months, median overal survival 14 months and median time to treatment failure 15 months. Toxicity was tolerable with 5 episodes of ifosfamide related CNS toxicity grade II, one ifosfamide related acute renal failure and 11 episodes of neutropenic fever. One patient died due to a neutropenic sepsis. No other non-hematological toxicity exceeding grade II was observed. Conclusion: IDx is a well tolerated and highly active chemotherapy regimen for first line treatment of soft tissue sarcoma, even in elderly patients. Randomized studies with this regimen are warranted.

\section{O423}

\section{An Open Label, Non-Comparative Phase II Study of Gemcitabine as Salvage Treatment for Patients with Pretreated Advanced Soft Tissue Sarcoma}

\section{Hartmann J.T. ${ }^{1}$, Oechsle K. ${ }^{1}$, Huober J. ${ }^{1}$, Jakob A. ${ }^{2}$, Azemar M. ${ }^{3}$,} Kanz L. ', Bokemeyer C.

${ }^{1}$ Universitätsklinikum Tuebingen, Germany, ${ }^{2}$ Klinikum Offenburg, Germany, ${ }^{3}$ Tumorbiologie Freiburg, Germany

Purpose: The number of effective cytotoxic agents for the treatment of patients (pts) with metastatic soft tissue sarcoma (STS) is limited, especially when pts have failed anthracyline-based chemotherapy. The aim of this trial was to evaluate the efficacy of gemcitabine in pretreated pts with STS Methods: Between 8/01 and 3/03 19 pts were eligible to enter this openlabel, non-comparative study. Gemcitabine was administered as a 30-minutes infusion at a dosage of $1000 \mathrm{mg} / \mathrm{m}^{2}$ on day 1,8 and 15 every 4 weeks. All patients had progressive disease during or shortly after an anthracycline-/ifosfamide- based regimen. The primary objective of the study was to determine the overall response rate (CR/PR). A two-stage study design was used. Results: Four of 19 pts did not start study treatment because of fulminant progression. Fifteen pts, median age 47 years (32 - 72), with various histological subtypes of STS were assessable. All pts had received at least one prior treatment regimen $(1-6)$ for metastatic disease containing anthracyclines $(14$ $\mathrm{pts} / 93 \%)$ and ifosfamide (12 pts/80\%). Extent of disease at study entry included local recurrence $(n=12)$, lung mets $(n=12)$, lymph node involvement $(n=5)$ and liver as well as bone mets $(n=3$ each). To date, a total of $62+$ cycles have been applied (median: $3 ; 1-18+$ ). Seven pts $(47 \%)$ had progressive disease at the first response assessment. One pt $(6 \%)$ attained a PR, and 7 pts $(47 \%)$ achieved disease stabilisations (SD). The reason for treatment stop was tumor progression in 13 pts $(87 \%)$. Two pts are still on treatment $(6$. respective 18. cycle). The median progression-free rate (PFR) was 3 mos (1-18+) and the median overall survival 6 mos (3-18+). Toxicity profile was favourable. $87 \%$ of the cycles have been applied without dose modification or delay. Grade 3 toxicities consisted of thrombocytopenia in 5 pts (33\%), leucopenia ( $2 \mathrm{pts} / 13 \%)$ as well as anemia, neutropenic infection, alopecia and flu-like symptoms (1 pts/7\% each). Conclusions: This series confirms that a considerable number of disease stabilisations in pretreated adult STS patients can be achieved with gemcitabine. The calculated PFR at 3 and 6 mos were $46.7 \%\left(\mathrm{CI}_{95 \%}, 21.4-71.9\right)$ and $13.3 \%\left(\mathrm{CI}_{95 \%}, 0-30.5\right)$. Considering response and progression-free rate as primary endpoints for phase II trials in STS, gemcitabine has moderate but defined efficacy in pretreated adult STS.

\section{O424}

\section{Treatment of Malignant Peritoneal Mesothelioma with Pemetrexed}

Karthaus M., Plahl A., Frieler F., Wert N., Maher G., Metzner D. Med. Klinik II, Ev. Joh. KH Bielefeld, Germany

Malignant pleural mesothelioma (MPM) is a rare and aggressive neoplasm of the lining of the lung. Rare reports are available for treatment and outcome of peritoneal mesothelioma only (AbM). Pemetrexed (ALIMTA), is a novel antifolate targeting key enzymes in purine and pyrimidine synthesis. Recently data from a randomized phase III have shown superior efficacy of pemetrexed/DDP vs DDP alone in MPM. No data of pemetrexed in AbM are available. In an open label trial efficacy and safety data of pemetrexed $\left(500 \mathrm{mg} / \mathrm{m}^{2}\right)$ $+/$-CDDP $\left(75 \mathrm{mg} / \mathrm{m}^{2}\right.$ ) or carboplatin (AUC 5) were studied in malignant peritoneal mesothelioma.

A total of 49 pts with mesothelioma with stage III/IV were observed between 12/02 and 04/04. Pts received pemetrexed based therapy including dexamethasone prophylaxis for skin rash on day -1 to +2 additionally. Folic acid 400 $\mu \mathrm{g}$ po daily, prior to and during study, and vitamin $B_{12} 1000 \mu \mathrm{g}$ i.m. q 9 wks in addition was administered to prevent adverse events. Study endpoints were time to progression (TTP), best response and safety. Results: Four pts (1 AbM, 1 MPM, 2 MPM+AbM) were excluded due to renal impairment $(n=1)$ or death prior to CTX $(n=3) .45$ mesothelioma pts $(34 \mathrm{~m} / 11 \mathrm{f})$ were treated from 12/2002 until 11/2003. Staging revealed AbM in 10 pts, MPM in 30 pts, while 5 pts had malignant mesothelioma on both sites of the diaphragma Initial combination was with DDP in 34 pts and carboplatin in 11 pts. Pemetrexed was administered in a median of 6 cycles (range $1-13$ ). Major toxicity $\left(\mathrm{WHO}>^{\circ} \mathrm{III} / \mathrm{IV}\right)$ was nausea and neutropenia. Response are presented in the following table.

\begin{tabular}{|l|c|c|c|}
\hline & $\begin{array}{c}\text { AbM } \\
(\mathrm{n}=10)\end{array}$ & $\begin{array}{c}\text { MPM + AbM } \\
(\mathrm{n}=5)\end{array}$ & $\begin{array}{c}\text { MPM } \\
(\mathrm{n}=30)\end{array}$ \\
\hline Male/Female & $8 / 2$ & $4 / 1$ & $22 / 8$ \\
\hline Mean Age & 64,2 & 60,8 & 69,4 \\
\hline $\begin{array}{l}\text { Pretreated } \\
\text { Patients }\end{array}$ & 3 & 3 & 13 \\
\hline $\begin{array}{l}\text { Pemetrexed } \\
\text { cycles/median }\end{array}$ & $70 / 6$ & $39 / 8$ & $156 / 6$ \\
\hline $\begin{array}{l}\text { RR } \\
(\mathrm{CR}+P R / S D)\end{array}$ & $3(30 \%) /$ & $3(60 \%) /$ & $10(33 \%) /$ \\
$9(40 \%)$ & $2(40 \%)$ & $9(27 \%)$ \\
\hline
\end{tabular}

Data regarding TTP, best response and safety will be presented at this meeting. Conclusion: Pemetrexed in combination with platinum is a well tolerable and active regimen for advanced peritoneal malignant mesothelioma. 
O425

Phase-II Study with Capecitabine and Celecoxib (CC) in Patients with Advanced Solid Tumors

Medinger M., Steinbild S., Baas F., Wiessner S., Frost A., Drevs J., Arends J., Strecker R., Unger C., Mross K. Tumor Biology Center at the Albert-Ludwigs-University Freiburg, Germany

Purpose: Cytotoxic agents are effecting tumor cells as well endothelial cells. The application of low dose continuous (metronomic) chemotherapy may optimize the effect on the tumor endothelial cells, acting like an unspecific anti-angiogenic regimen. Capecitabine is a prodrug of 5-FU. Because of high concentrations of thymidilatsynthase in proliferating endothelial cells, metronomic application of capecitabine might inhibit angiogenesis. Celecoxib, a selective cyclooxygenase-2 (COX-2) inhibitor, is known to have an apoptotic effect on tumor cells as well as an anti-angiogenic effect on tumor endothelia cells. The objectives of the trial was to evaluate the antitumor activity and toxicity of continuous treatment with the combination of CC in patients (pts) with advanced cancer. Methods: Pts were treated with capecitabine $500 \mathrm{mg}$ bid and celecoxib $400 \mathrm{mg}$ bid continuously until progressive disease or toxicity occurred. Pts with advanced or metastatic cancer were included in the trial. 36 patients ( 16 female, 20 male) were treated with CC. Mean age was 58.3 years. 1 cycle was defined as a period of 4 weeks. Assessment of tumor size was performed before, after 1 and 3 cycles and then every $2^{\text {nd }}$ cycle. Dynamic contrast-enhanced MRI (DCE-MRI) was used for two reasons: 1. to evaluate tumor vessel permeability and thus the anti-angiogenic effect of $\mathrm{CC}$; 2. to assess the tumor size. Pts were evaluable for response when they were treated at least 3 cycles. Results: 2 pts had stable disease (SD) for 6 cycles, 1 pt had SD for 4 cycles, and $1 \mathrm{pt} \mathrm{had} \mathrm{SD} \mathrm{for} 3$ cycles, then progressive disease (PD). 8 pts had PD after 3 cycles. 13 pts did not complete 3 cycles because of $\mathrm{PD}$ and therefore were not evaluable for response. 10 pts are ongoing in cycle 1-3. Evaluation of response in these pts will be performed after completion of 3 cycles. Regarding toxicity, 2 pts had an increase in creatinine levels, possibly due to celecoxib treatment, and were excluded from the study. 2 pts developed a mild hand-foot-syndrome. All toxicities were reversible Conclusions: In general, therapy with CC was well tolerated and showed potential antitumor activity. Cancer control (no change, stable disease) is the main goal of a successful anti-angiogenic therapy. Although CC is able to reach cancer control in some patients, patients with aggressive tumor progression doesn't seem to profit from this drug combination. The DCE-MRI was a valuable tool to track antiangiogenic effects of the $\mathrm{CC}$ treatment.

\section{Scientific Session: Aggressive Non-Hodgkin's Lymphoma}

\section{3}

\section{Chemotherapy of DLBCL: New standards?}

\section{Hartmann F, Pfreundschuh M für die Deutsche Studiengruppe hoch-maligne Non-Hodgkin-Lymphome (DSHNHL)}

Medizinische Klinik I, Universitätskliniken des Saarlandes, Homburg, Germany

While CHOP given every 3 weeks remained the standard treatment of aggressive lymphomas for more than 25 years, it was recognized that the prognosi of a given patient is dependent on pretherapeutically identifiable prognostic factors, which led many cooperative groups to distinguish three populations of patients with respect to therapy.

- In elderly patients ( $>60 \mathrm{yrs}$.) the DSHNHL succeeded in improving treatment results (CR-rates $+16 \%$, EFS and OS after 5 years $+11 \%$ and $+13 \%$, respectively) by shortening time intervals between chemotherapy cycles from 3 to 2 weeks (CHOP-14). Similar improvements after only 3 years (EFS + 18 $\%$, OS $+11 \%$ ) were achieved by the GELA by adding the monoclonal antiCD20 antibody rituximab to the classical 3-weekly CHOP (R-CHOP-21). While the ECOG 4494 trial for elderly patients confirmed to some degree the efficacy of rituximab in induction therapy, it disproved the value of rituximab maintenance treatment thereafter. The high-priority question in elderly patients with aggressive lymphomas is the question if the combination of CHOP-14 and rituximab can further improve treatment results and will be answered by the DSHNHL 1999-1 (Ricover 60) trial, expected to close enrolment of $>1200$ patients at the end of this year.

- In young low-risk patients (IPI 0,1), DSHNHL achieved a significan improvement of treatment results (CR-rate, EFS) by the addition of etoposide to CHOP (CHOEP) and for CHOEP-14 also an increased overall survival (85 vs. $75 \%$ after 5 years). Further strategies to improve outcome were moderate escalation of cytostatic drugs (1.3-1.8 fold: "HighCHOEP") or the addition of rituximab. While interim analyses showed no advantage for dose escalated HighCHOEP compared to CHOEP-21 in the 1999-2 trial, the addition of rituximab to a CHOP-like regimen in young low-risk patients led to significant improvements in CR-rate, EFS and survival after a median follow-up of barely two years (MINT-trial).

- In young high-risk patients (IPI $\geq 2$ ) the situation is problematic: since highdose strategies failed to prove their superiority in terms of overall survival prospectively within 10 randomized trials (however, in two trials there was an advantage in a retrospective subgroup analysis of high-risk patients) and the strategies mentioned above which resulted in improvement of treatment results in other patient subpopulations have not been tested in this group, it is hardly justifiable to treat these patients outside of a prospective clinical trial. Within the trial 2002-1, DSHNHL compares 8 cycles of CHOEP-14 + rituximab as a maximal dose dense conventional treatment with "MegaCHOEP" + rituximab, an innovative high-dose regimen employing triple-transplant upfront and the most active cytostatic drugs in a considerably escalated dose as conditioning regimens.

\section{Poster Session: Angiogenesis}

\section{P454 \\ Small Rho GTP Binding Proteins Regulate Vascular Endothelial Growth Factor-2 Expresssion IN Endothelial CelLS}

\section{Meissner $M^{1}$, Stein $M .{ }^{1}$, Kaufmann $R^{1}{ }^{1}$, Gille $J^{1,2}$}

${ }^{1}$ Dept. of Dermatology, J.W. Goethe-Universität, Frankfurt/M. Germany, ${ }^{2}$ Dept. of Molecular Biology, Max-Planck-Institut, Bad Nauheim, Germany

Recent evidence suggests, that rho-family small GTPases play a significant role in the regulation of different endothelial cell functions, implicated in capillary network formation. Whereas Rho-family small GTPases are primarily known to control actin-based motility processes, RhoA, Rac1 and Cdc42 have been shown to regulate other cellular activities as well, such as membrane transport and gene transcription. As vascular endothelial growth factor receptor-2 (VEGFR2) expression is necessary for angiogenic responses to occur, we hypothesized that Rho-family small GTPases may affect VEGFR2 expression by cultured endothelial cells (ECs). Overexpression of dominant negative mutants N17Rac1 and N19RhoA were seen to significantly inhibit VEGFR2 protein expression by HUVEC, whereas transfection of mutant N17Cdc42 failed to affect VEGFR2 levels. As N17Rac1 and N19RhoA also supressed VEGFR2 mRNA accumulation, we subsequently examined their effects on VEGFR2 transcriptional activation. Analyses of a different $5^{\prime}$-deletional VEGFR2 promoter-based reporter gene constructs revealed that inhibition by N17Rac1 and N19RhoA is conveyed by distinct gene regulatory elements. Whereas N17 Rac1-mediated supression is confined to a GC-rich region between bp -77 and -60, N19RhoA-mediated inhibition appears to be conferred by an element located between bp -225 and -160 , harboring a consensus E-box binding site. We were able to show by EMSA analysis, that constitutive Sp1-dependent DNA binding to the GG-rich region is decreased by N17Rac1 transfection, indicating that inhibition of rac1 may interfere with Sp1-dependent VEGFR2 transcription. Therefore, different members of the Rho-family small GTPases exert diverse effects on VEGFR2 expression. Significantly, inhibition of VEGFR2 transcription by N17Rac1 and N19RhoA involves distinct molecular mechanisms as differen elements seem to be engaged. In conclusion, control of capillary network formation by Rho-family small GTPases may likely be mediated in part via modulation of VEGFR2 expression.

P455

\section{Imatinib and Microvascular Endothelial Cell Function}

Raida M., Kleeberg S., Günther C., Niederwieser D.

Div. of Hematology and Oncology, University of Leipzig, Germany

Purpose: Imatinib is a tyrosine kinase inhibitor with c-kit, c-Abl, and PDGF beta-receptor activity that has been shown to reduce increased bone marrow vascularity and Bcr-Abl mediated VEGF secretion in patients with chronic myeloid leukaemia (CML). Microvascular endothelium is involved in the 
regulation of trafficking, differentiation, and homing of stem cells within the haematopoietic microenvironment. It has been suggested that imatinib influences angiogenesis through an anti-migratory and anti-proliferative effect on vascular smooth muscle cells via the inhibition of the PDGF beta-receptor phosphorylation. In our study we analysed the effect of imatinib on proliferation and function of microvascular endothelial cells. Methods: The effects of imatinib at concentrations varying between 0.1 and $50 \mathrm{mM}$ on human microvascular endothelial cells (HMVEC) were observed in 4 in vitro assays. Endothelial proliferation was detected with a cell viability assay based on the ability of living cells to convert a redox dye (resazurin) into a fluorescent end product (resorufin). Endothelial function was assessed by the ability of HMVEC to form tube networks in growth factor reduced matrigel. Tube formation was detected by phase contrast microscopy and recorded with a digital camera. Endothelial cell migration was analysed using the scratch wound healing assay and the Boyden chamber chemotaxis assay. Endothelia cell basal medium was supplemented with the growth factors FGF-B, VEGF, IGF-1, EGF. Endothelial cells that migrated through the pores of the membrane to the bottom chamber were stained and counted in 20 random fields. Tube formation and scratch wound healing were quantified with image analysis software. Results: Imatinib treatment showed no significant effect on HMVEC proliferation in therapeutic concentrations $(0.1-10 \mathrm{mM})$. Interestingly, we could observe that imatinib increased the endothelial tube and network formation on matrigel. However, the migration of HMVEC was clearly suppressed in the wound healing migration as well as in the Boyden chamber chemotaxis assay. Conclusions: These data suggest that the angiostatic property of imatinib also involves anti-migratory effects on endothelia cells without affecting their ability to proliferate and to form tube networks. Therefore, imatinib in addition to its efficacy in the antineoplastic treatment of CML and certain cancers might also prove to be clinically useful in diseases characterized by unregulated angiogenesis.

\section{Poster Session: Molecular Genetics}

\section{P456}

\section{Localization of BCR-ABL in Philadelphia-Chromosom Positive Blasts is Dependent on its Aberrant Kinase Activity}

\section{Güller S., Beissert T., Hoelzer D., Ruthardt M., Ottmann O.G. University Hospital Frankfurt, Germany}

Purpose: The chromosomal translocation $\mathrm{t}(9 ; 22)$ giving rise to the Philadelphia $(\mathrm{Ph})$ chromosome aberrantly fuses BCR sequences to the non-recepto tyrosine kinase $\mathrm{c}-\mathrm{ABL}$. The presence of the BCR oligomerisation interface in the fusion protein leads to its tetramerisation and to a constitutive activation of the ABL tyrosine kinase. Multiple signalling pathways such as the RAS pathway or JAK-STAT kinases activated by BCR-ABL transform haematopoietic cells and lead to the malignant phenotype of $\mathrm{Ph}$-chromosome positive CMLs and ALLs. BCR-ABL is expressed in about $95 \%$ of CML and $20-25 \%$ of adult ALL cases. The specific ABL kinase inhibitor STI571 induces apoptosis in BCR-ABL transformed cells, leads to complete remission in chronic phase CML-patients and has a remarkable but not durable effect in CML-blast crisis or in Ph-chromosome positive ALL patients. In this study we investigated the cellular localization of BCR-ABL and its dependency on the kinase activity and the colocalization with the BCR-ABL adapto proteins GRB-2 and SHC. Methods: We treated cell lines endogenously expressing BCR-ABL (BV173, Sup-B15) and cell lines infected with BCR ABL constructs (BaF3) as well as primary cells from a patient with CML blast crisis with $0,5 \mu \mathrm{M}$ STI571. As a control we investigated cells infected with a kinase deficient BCR-ABL mutant. At different time points the cell were fixed on cover slides. The subcellular localization of BCR-ABL was analysed by immunofluorescence with an ABL specific antibody. Colocalization of ABL with GRB-2 or SHC were studied by co-immunofluorescen stainings with ABL and GRB-2 or SHC specific antibodies. Pictures of the cells were taken by confocal laser scan microscope. Results: We found that $i$ $\mathrm{BCR}-\mathrm{ABL}$ is localized in dots whereas wildtype $\mathrm{ABL}$ is distributed diffusely among cytoplasma ii) the typical BCR-ABL staining pattern is detected in endogenously BCR-ABL expressing cell lines, in cells infected with BCR $\mathrm{ABL}$ constructs and in primary $\mathrm{Ph}$-chromosome positive cells iii) the localization of $\mathrm{BCR}-\mathrm{ABL}$ is perinuclear iv) treatment of $\mathrm{Ph}$-chromosome positive cells with STI571 leads to redistribution of ABL from BCR-ABL dots to microspeckled pattern v) the same effect can be achieved in transfected cells with a BCR-ABL construct lacking the tyrosine kinase domain vi) there is no colocalization of BCR-ABL with the adaptor proteins GRB-2 or SHC before or after treatment with STI571 and vii) the effect of STI571 is irreversible. Conclusions: The results of the localization studies indicate that the staining pattern of $\mathrm{ABL}$ and BCR-ABL differ completely and that the typical BCRABL pattern strictly depends on the aberrant tyrosine kinase activity of the fusion protein. The responsible mechanism is unknown but further investigations may elucidate the formation of BCR-ABL dots and involvement of the kinase activity and its downstream targets.

\section{P457}

\section{The MYST Domain Histone Acetyltransferase TIP60 Acts a Transcriptional Corepressor for the Putative Tumor Suppressor Gene ETV6(TEL)}

\section{Zhang C.D., Bohlander S.K.}

Department of Medicine III, Klinikum Großhadern and Clinical Cooperative Group Leukemia, GSF, Munich, Germany

ETV6 (TEL) is a member of the Ets family of trancription factors and is involved in many translocations forming different fusion proteins (e.g ETV6/AML1) in both myeloid and lymphoid leukemias as well as in some solid tumors. Because the non-rearranged allele of ETV6 is frequently deleted in ALL, ETV6 might be a tumor suppressor gene. ETV6 acts as a transcriptional repressor and interacts with several transcriptional co-repressors (mSin3, Smrt, N-CoR, L(3)MBT). We previously reported that ETV6 interacts with the MYST domain histone acetyltransferase (HAT) TIP60. The highly conserved MYST domain of TIP60 includes a zinc finger and an acetyl co-enzyme A binding site, which acetylates histone $\mathrm{H} 4 / \mathrm{H} 3 / \mathrm{H} 2 \mathrm{~A}$ in vitro. TIP60 functions as a transcriptional co-activator when binding to HIV1 Tat, class I nuclear hormone receptors and App (B-Amyloid Precursor Protein) or as a transcriptional co-repressor when interacting with STAT3, CREB and ZEB (Zinc finger E.Box-binding protein). TIP60 also plays a role in DNA repair and apoptosis. Other members of the MYST family (MOZ and MORF) form fusion genes with CBP, P300 or TIF2 in acute myeloid leukemia.

We were able to show that the interaction between ETV6 and TIP60 is critically dependent on the $\mathrm{C} 2 \mathrm{HC}$ zinc finger and on the acetyltransferase activity of TIP60 and that complicated acetylation-dependent conformational changes might be necessary for this interaction. When tagged with cyan or yellow fluorescent proteins, ETV6 and TIP60 could be shown to interact in the nucleus of transiently transfected cells. However, the functional significance of this interaction was not evident. Nordentoft and Jorgensen (Biochem J $374: 165-173,2003$ ) reported that TIP60 acts as a corepressor of a GAL4 ETV6 fusion protein. We were able to repeat these results using the GAL4ETV6 fusion protein and a luciferase reporter gene that was under the contro of GAL4-DNA binding sites. Furthermore, using a luciferase reporter under the control of the ETV6-responsive stromelysin-1 promoter (pTR334) we could show that transcriptional repression meditated by ETV6 is enhanced by the co-expression of TIP60. The stromelysin-1 promoter system is more physiologically relevant because here ETV6 uses its own DNA binding domain. An acetyltransferase deficient mutant of TIP60 had reduced corepressor activity. These results suggest that the MYST domain HAT TIP60 is an important player in leukemogensis.

\section{P458}

\section{Checkpoint Deficiency Increases Telomere Dysfunction and Genetic Instability in Tumor Cells Following Telomeras Inhibition}

Pantic M. ${ }^{1}$, Zimmermann S. ${ }^{1}$, EIDaly $H^{1} .{ }^{\text {, Popp }}$ S. $^{2}$, Boukamp $P .{ }^{2}$, Waller C. ${ }^{1}$, Martens U.M.

${ }^{1}$ University Hospital Freiburg, Germany, ${ }^{2}$ German Cancer Research Center, Heidelberg, Germany

Purpose: Telomerase is the ribonucleoprotein enzyme that maintains telomeres of eukaryotic chromosomes. Activation of telomerase is a common feature of the majority of human cancers and inhibition of this enzyme has been proposed as novel target for cancer therapeutics. Here, we wanted to evaluate whether growth arrest and genetic stability is affected following telomerase inhibition in tumor cells with different checkpoint status. Methods: Telomerase activity was completely abolished in the $\mathrm{p} 53^{+/+}$colon cancer cell line HCT-116 using ectopic expression of a dominant-negative mutant of hTERT (DN-hTERT). In addition, telomerase activity was suppressed in two derivative cell lines which were $\mathrm{p} 53^{-/-}$and $\mathrm{p} 21^{-/}$, respec- 
tively. Results: Telomere erosion was observed leading to a delayed proliferation arrest in a all selected subclones $(n=10)$. Interestingly, the lag period varied based on the genetic background: whereas $\mathrm{p} 53^{+/+}$cells $(n=3)$ stopped proliferation uniformly after 40 population doublings (PDs), growth arrest occurred between 38 and 59 PDs in p53 ${ }^{-1-}(\mathrm{n}=4)$ and between 40 to 59 PDs in $\mathrm{p} 21^{-1}$ cells $(\mathrm{n}=3)$. Although Q-FISH and M-FISH analysis revealed that all subclones showed increased rates of telomere dysfunction such as signal free ends and end-to-end fusions, the level was strikingly pronounced in $\mathrm{p} 53^{-/}$and p $21^{-1-}$ cells, respectively. Furthermore, we observed a higher rate of hyperdiploidy in checkpoint deficient cells without telomerase activity. Conclusions: These data suggest that the p53 and p 21 checkpoint status is critical for the genetic stability in tumor cells following telomere dysfunction. Importantly, the resulting genetic crisis appears to be incompatible with cell proliferation which justifies the concept of telomerase inhibition for cancer therapy.

\section{P459}

\section{Enhancers of the RUNX2 Gene Active in Human Prostate Cancer}

Stock M., Huber I., Enger S., Otto F.

University of Freiburg, Medical Centre, Dept. of Internal Medicine I,

Freiburg, Germany

Purpose: The transcription factor RUNX2 has been accepted to be the key regulator of osteoblastic development. Recently however, several reports have been published suggesting a role for RUNX2 in different types of tumours, in particular in those that can form bone metastases. Prostate cance is such a type of tumour. We focused our studies on the signalling pathways that lead to ectopic RUNX2 expression. Our knowledge of the control of RUNX2 gene expression is mainly confined to external signals and to trans acting factors. Cis acting genetic elements however, are poorly understood. Thus far studies on the two promoters of the RUNX2 gene explain only a fraction of its observed physiological expression pattern. Hence, we conclude that there are additional regulatory elements required for expression control of RUNX2 at least in some tissues. Methods: For sequence comparison NCBI's Blast programmes were used. Mouse counterparts of the conserved genetic elements CR3, CR4, and CR5 were amplified by PCR from genomic mouse DNA and cloned into pGL-3 promoter (Promega). RUNX2 expression was assayed by RT-PCR. Mesenchymal cells were transfected with Fugene 6 transfection reagent (Roche), prostate cancer cells were transfected with Transfast (Promega). Enhancer activity was measured applying Dua Luciferase Assay System from Promega according to the manufacturer's protocol. Results: We previously screened the RUNX2 loci of human and Fugu rubripes for highly conserved non-coding sequences that might represent regulatory elements. This screen revealed the two promoter sequences (here termed CR1 and CR2) and further three conserved sequences: CR3, CR4 and CR5. In the present study we functionally analysed CR3-5 by generating reporter constructs as enhancers and a firefly luciferase gene driven by an SV40 minimal promoter. With these reporter constructs the enhance potential of the respective elements were analysed in mesenchymal and prostate cancer cells. This study provides first data supporting the assumption that CR3 and CR5 in contrast to CR4 are functional enhancer elements. While CR3 displays its enhancer activity in a constitutional way, CR5 enhance activity is linked to RUNX2 expression. Conclusions: We conclude, that CR5 is a good candidate for a RUNX2-specific enhancer active in prostate cancer cells. CR3 seems to be a constitutional enhancer of transcription.

\section{P460}

\section{Analysis CALM/AF10 Fusion Gene Positive Leukemias: HOX Gene Deregulation and High Incidence of CALM/AF10 Fusions in T-ALL with TCR $\gamma / \delta$ Rearrangement}

Krause $A^{1,2}$, Kohlmann A. ${ }^{1}$, Schnittger $S .{ }^{1}$, Schoch $C .{ }^{1}$, Haferlach T. ${ }^{1}$, Mecucci C. ${ }^{3}$, Ludwig W.D. ${ }^{4}$, Bohlander S.K. ${ }^{1,2}$ ${ }^{1}$ Med III, ${ }^{2}$ GSF, Clinical Cooperative Group „Leukemia“, Klinikum Großhadern, München, ${ }^{3}$ Ematologia, Policlinico Monteluce, Perugia, Italy, ${ }^{4}$ Immunological Marker Laboratory, Charite University Hosptial, Berlin, Germany

The $\mathrm{t}(10 ; 11)(\mathrm{p} 13 ; \mathrm{q} 14)$ resulting in the $C A L M / A F 10$ fusion gene is found in undifferentiated leukemia, acute myeloid leukemia, acute lymphoblastic leukemia and malignant lymphoma. The CALM/AF10 fusion protein was reported to be the most common fusion protein in T-ALL with TCR $\gamma / \delta$ rearrangement. $C A L M / A F 10$ is associated with a poor prognosis.

We have analyzed samples from ten patients with different types of leukemia: case 1 (AMLM2), case 2 (AML M0), case 3 (Pre T-ALL), case 4 (AL, unclear), case 5 and case 6 (ALL), case 7 and 8 (ProT-ALL) ), with a t(10;11) which did not show involvement of the MLL gene. In addition, we analysed a series ten patients with T-ALL with TCR g/d rearrangement. The samples were analysed for the presence of the CALM/AF10 and AF10/CALM mRNA by RT-PCR and sequence analysis. cDNA of five patients was analysed using oligolnucleotide microarrays representing 33000 different genes.

All ten patients with reported $\mathrm{t}(10 ; 11)$ were positive for the $C A L M / A F 10$ fusion. We found two different breakpoints in CALM at nucleotide 1926 and 2091. In addition, a new exon, with 106 bases after nt 2091 was found. In AF10 four breakpoints were identified: at position 424, 589, 883 and 979. Of the ten T-ALL patients with TCR $\gamma / \delta$ rearrangement from which the karyotype was unknown, two were positive for the CALM/AF10 transcript, confirming previous results (Asnafi et al, 2003). In these two patients the breakpoint in CALM was at nucleotide 1926 and 2091. In AF10 the breakpoint was at nucleotide 883 . In five patients, it was also possible to amplify the reciprocal $A F 10 / C A L M$ fusion transcript (case 1, 3, 4, 5 and 6). There was no corrrelation between disease phenotype and breakpoint location. Preliminary analysis of microarray expression profiling of five of these cases revealed high expression levels of the HOX genes. The overexpression of HOX genes seen in these CALM/AF10 positive leukemias is reminiscent of the pattern seen in leukemias with rearrangements of the $M L L$ gene, norma karyotypes and komplex aberrant karyotypes suggesting a common effector pathway (i.e. HOX gene deregulation) for these diverse leukemias. It is known that alhambra, the Drosophila homologue of AF10, can act on polycomb group responsive elements. It is thus conceivable that the CALM/AF10 fusion proteins acts in a dominant negative fashion on wildtype AF10 function, relieving the repression that is presumably normally exerted by AF10 on the expression of the HOX genes.

P461

\section{The Multidomain Bcl-2 Homolog Bax but not Bak Mediates Synergistic Induction of Apoptosis by TRAIL and 5-FU through the Mitochondrial Apoptosis Pathway}

von Haefen C. ${ }^{1}$, Gillissen B. ${ }^{1}$, Hemmati P. G. ${ }^{1}$, Wendt J. ${ }^{1}$, Güner $D .{ }^{2}$ Mrozek A. ${ }^{1}$, Belka C. ${ }^{3}$, Dörken B. ${ }^{1}$, Daniel P. ${ }^{1,4}$

${ }^{1}$ Department of Hematology, Oncology and Tumor Immunology, University Medical Center Charité, Humboldt University, Berlin, Germany, ${ }^{2}$ Department of Radiation Oncology, University Medical Center Charité, Campus Mitte, Humboldt University, Berlin, Germany, ${ }^{3}$ Department of Radiation Oncology, Medical Faculty, EberhardKarls-University, Tübingen, Germany, ${ }^{4}$ corresponding author

The death ligand TRAIL has been suggested as a suitable biological agent for the selective induction of cell death in cancer cells. Moreover, TRAIL synergizes with DNA damaging therapies such as chemotherapeutic drugs or ionising irradiation. Here, we show that synergy of TRAIL and 5-fluorouracil (5-FU), i.e. cross-sensitization between TRAIL and 5-FU for induction of apoptosis, entirely depends on Bax proficiency in human DU145 and HCT116_carcinoma cells. DU145 prostate carcinoma cells that have lost Bax protein expression due to mutation fail to release cytochrome $\mathrm{c}$ and to activate caspase-3 and -9 when exposed to TRAIL and 5-FU. In contrast, TRAII sensitized for 5-FU-induced apoptosis and vice versa upon reconstitution of Bax expression. Sensitization occurred through a caspase- 8 dependent pathway and was associated with Bid cleavage. Isobolographic analyses of ED50 doses for 5-FU at increasing TRAIL concentrations showed a clear synergism of TRAIL and 5-FU in Bax-expressing cells. In contrast, the effect was merely additive in DU145 cells lacking Bax. Notably, DU145 and other cells having lost Bax such as the frequently employed HCT116 Bax k.o. cell line still express Bak. This indicated that Bak is not sufficient to mediate cross-sensitization and synergism between 5-FU and TRAIL. Stable overexpression of Bak in DU145 sensitized for epirubicin-induced apoptosis but failed to confer synergy between TRAIL and 5-FU. Moreover, we show by the use of EGFP-tagged Bax and Bak that TRAIL and 5-FU synergistically trigger oligomerization and clustering of Bax but not Bak. These data clearly establish distinct roles for Bax and Bak in linking the TRAIL death receptor pathway to the mitochondrial apoptosis signaling cascade and delineate a higher degree of specificity in signaling for cell death by multidomain Bcl-2 homologs. 
P462

No Influence of NOD2/CARD 15 Mutations with Transplant-Related Mortality and Acute GVHD in Patients who Received an Intestinal Bacterial Decontamination using Metronidazole and Ciprofloxacin after allogeneic Stem Cell Transplantation

\section{Elmaagacli A.H., Steckel N., Koldehoff M.}

Department of Bone Marrow Transplantation, University Hospital of Essen, Germany

Mutations of the NOD2/CARD14 gene have been associated with an increased incidence of Crohns disease due to a diminished NF-kB response to bacterial cell wall products. Moreover, it was reported that mutations of this gene loci might be associated also with an increased risk for TRM and severe GVHD in patients who underwent allogeneic blood stem cell transplantation. Here we analyzed 201 patients and their respective donors for NOD2/CARD15 mutations and correlated the results with the incidence of acute GVHD. Moreover, we evaluated if the occurrence of NOD2/CARD15 gene mutations are accompanied with an increased risk for transplant-related mortality. Mutated alleles were observed in $17 \%$ of the patients and $16 \%$ of the patients. The estimates for one-year transplant-related mortality did not differ significantly between patient/donor pairs without mutations $(27 \%)$, patients with mutated alleles $(13 \%)$, donors with mutated alleles $(22 \%)$, or donor/patient with mutated alleles (18\%). Also no significant differences in the incidence of acute GVHD (grade 1-4) and intestinal GVHD were seen in the four groups. But patient/donor pairs with mutated alleles seemed to have a slightly higher incidence of severe acute GVHD (grade 3-4) compared to patient/ donor pairs without had mutated alleles $(n=6$ of $11,55 \%)$ versus patient/donor pairs without mutations $(35 / 145,24 \%)$. Multivariate analyses including all potential factors, which might have influence on the outcome, revealed that mutations of NOD2/CARD15 had no influence either on the occurrence of acute GVHD, nor on the occurrence of transplant-related mortality. Since all patients had received an intestinal bacterial decontamination using Metronidazole and Ciprofloxacin after allogeneic stem cell transplantation it might be speculative if the reduction of concentrations of anaerobic bacteria in the intestinum might have protected patients from the occurrence of increased TRM or incidence of GVHD. Higher concentrations of anaerobic bacteria are associated with an increased risk for severe GVHD as reported earlier.

\section{P463}

The 638 G>A Polymorphism of the Sulfotransferase 1 A1 Gene and Breast Cancer Risk

Langsenlehner U. ${ }^{1}$, Krippl P. ${ }^{1}$, Eder $T^{2}{ }^{2}$, Weitzer $W .{ }^{1}$, Samonigg $H .{ }^{1}$ ${ }^{1}$ Department of Internal Medicine, Division of Oncology, Medical University Graz, Austria, ${ }^{2}$ Department of Radiooncology, Medical University Graz, Austria

Purpose: Sulfotransferase 1A1 (SULT1A1) is involved in the bioactivation and detoxification of a variety of xenobiotic compounds. A common arginine (R) to histidine $(\mathrm{H})$ variation at amino acid position 213 influences SULT1A1 activity and has been suggested as a risk factor for a variety of cancers Methods: To investigate the role of this polymorphism for breast cancer risk, SULT1A1 genotype was determined in 500 women with clinically verified breast cancer and 500 female age-matched healthy control subjects. Genotyping was done by a 5'-nuclease assay $\left(\mathrm{TaqMan}^{\mathrm{TM}}\right)$. Primer and probe sets were designed and manufactured using Applied Biosystems 'Assay-byDesign' custom service. P-values were calculated by $\chi^{2}$ test using SPSS 10.0 All tests were two-sided, threshold for significance was $\mathrm{P}<0.05$. Results: Frequencies of heterozygous (controls: $42.5 \%$; patients: $50.2 \%$ ) or homozygous (controls: $12.6 \%$; patients: $9.4 \%$ ) carriers of the $213 \mathrm{H}$ variant were not significantly different between groups. The odds ratio of the SULT1A1 $\mathrm{R} 213 \mathrm{H}$ variant was 1.20 (95\% confidence interval $0.94-1.55)$ in a dominant model ( $\mathrm{RH}+\mathrm{HH}$ versus $\mathrm{RR})$ and $0.72(95 \%$ confidence interval $0.48-1.08)$ in a recessive model ( $\mathrm{HH}$ versus $\mathrm{RH}+\mathrm{RR}$ ), respectively. The SULT1A1 genotype was furthermore not associated with tumor size, histological grading, estrogen or progesterone receptor status and age at diagnosis. The SULT1A1 $213 \mathrm{H}$ variant was associated with the presence of lymph node metastases $(\mathrm{p}=$ 0.002). Conclusion: We conclude that the SULT1A1 R213H polymorphism is not a general risk factor for breast cancer, but may be involved in lymph node metastazing in breast cancer patients. Further prospective studies should be performed to analyze this potential role of the SULTA1 polymorphism for lymph node metastasis development.

\section{P464}

\section{In Multiple Myeloma, Chromosomal Abnormalities Involving the DKK1 Gene Locus (10q11) are Rarely Identified by FISH}

\author{
Wendl C., Döhner H., Liebisch P. \\ University Hospital UIm, Germany
}

Introduction: In multiple myeloma (MM), a number of secreted factors, such as RANKL and osteoprotegerin, are involved in the pathogenesis of bone destruction. In a recent gene expression profiling study, overexpression of DKK1, an inhibitor of osteoblast differentiation, has been demonstrated to be associated with the presence of lytic bone lesions in patient (pts.) with MM (Tian et al., 2003). The mechanisms leading to DKK1 overexpression in this disease remain unclear. The DKK1 gene resides to chromosome band 10q11. In chromosome banding or comparative genomic hybridization studies, chromosome 10 has not been reported to be a frequent site of genomic changes, such as genomic imbalances or reciprocal translocations. However, both techniques have limitations, especially with respect to their spatial resolution. To our knowledge, chromosome band 10q11 has not been studied yet for chromosomal aberrations using interphase FISH. Aim: To determine whether or not interphase FISH can reveal chromosomal abnormalities, e.g. high-level amplifications or chromosomal translocations, involving the DKK1 locus in clonal plasma cell disorders. Material and methods: Bone marrow specimen from 47 pts. (MM n=40, MGUS $n=7$ ) obtained during routine diagnostic procedures have been analysed in this study so far. Among the MM pts., 18 had advanced focal bone lesions as determined by conventional radiographs, while 22 pts. had no or only one osteolysis. Tri-color FISH using BAC probe RP11-346D6 (181 kbp) containing the DKK1 gene was applied as previously described. Results: No genomic change involving chromosome band 10q11 was identified among the 7 pts. with MGUS. Among the 40 pts. with MM, no high-level amplifications were found, while 5 pts. (12.5\%) exhibited extra copies involving chromosome band $10 \mathrm{q} 11$ (trisomy $\mathrm{n}=4$, tetrasomy $\mathrm{n}=1$ ). Additional FISH data indicated tetraploidy in 2 of these 5 pts. In 2 of $40 \mathrm{MM}$ pts. (5\%), a monoallelic deletion of chromosome band $10 \mathrm{q} 11$ was found There was no evidence for translocations breakpoints involving the DKK1 locus, i.e. disruption of BAC probe RP11-346D6. Conclusion: In MM, chromosomal aberrations detectable by FISH rarely involve the DKK1 gene locus at chromosome band $10 \mathrm{q} 11$.

P465

Phenotype Analysis of Mice Deficient in TLE3 Expression

Gasperowicz M., Johner C., Joos Y., Klemm N., Otto F. Department of Internal Medicine, Division of Hematology/Oncology, Albert-Ludwigs University Freiburg, Germany

AML/RUNX transcription factors are involved in many developmental processes in the organism. Three members of AML/RUNX family have been described. One of them, RUNX1/AML1, takes part in hematopoietic development. Haploinsufficiency of this transcription factor leads to the autosomal dominant heritable "familial platelet disorder with a predisposition to acute myelogenous leukemia" (FPD/AML). It is also involved in chromosomal translocations in acute leucemias (e.g. t8;21). The second member of the family, RUNX2, is a key factor of osteogenesis, and the third one, RUNX3, is important for neuronal development. All RUNX proteins are considered to be transcriptional activators, however they can be converted to transcriptional repressors by interaction with corepressor proteins, e.g. members of TLE family. It was shown that TLE family members interact with RUNX transcription factors in vitro and downregulate the expression of their target genes. They are also coexpressed in certain tissues. The restricted expression pattern of one member of TLE family, TLE3, points to a defined, specific interactions of this protein with RUNX transcription factors in vivo. To investigate the physiological relevance of TLE3 with respect to RUNX function we have generated a mouse strain deficient of TLE3 protein. Preliminary studies have shown that TLE-3 knock-out embryos are underdeveloped and die between e14.5 and e15.5. Additionally, for the disruption of the Tle3 gene we have used the targeting cassette containing a beta-galactosidase reporter gene. This strategy allowed us to map the expression pattern of Tle3 gene during embryogenesis and compare it with known expression domains of RUNX transcription factors. 


\section{P466}

\section{Gene Expression Profiling of Granulocytes of CML Patients Using 30k-Arrays}

\section{Marschon R. ${ }^{1}$, Amberger $A .{ }^{2}$, Margreiter $R^{2}{ }^{2}$, Webersinke G. ${ }^{1}$ Labor für Molekularbiologie und Tumorzytogenetik; Krankenhaus der Barmherzigen Schwestern, Linz, Austria, ${ }^{2}$ Department of Metabolism and Bioenergetics of Cancer, Tiroler Krebsforschungsinstitut, Innsbruck, Austria}

Purpose: Approximately $95 \%$ of patients with chronic myeloid leukemia (CML), a clonal hematopoietic stem cell disorder, show the reciproca translocation $\mathrm{t}(9 ; 22)(\mathrm{q} 34 ; \mathrm{q} 11)$. This so called Philadelphia chromosome leads to a constitutive activation of the abnormal tyrosine kinase resulting from the bcr-abl fusion-gene. Course of this disease is divided in three phases: chronic phase, accelerated phase and finally blast crisis. Although increase of bcr-abl genes on the transition to blast crisis can be well detected by real-time PCR molecular events leading to the progression of CML are still poorly understood. Therefore gene expression studies using array technology could be a powerful method for finding further prognostic and therapeutic targets. Methods: Granulocytes of peripheral blood from five untreated patients with primary diagnosis of CML and the molecular marker bcr-abl, as well as from control patients, were separated by CD 33/CD15 positive antibody conjugated magnetically beads. Following RNA isolation using a guanidinium isothiocyanate method and amplification according to Eberwein, cRNA was fragmented, labeled and hybridized to 30k oligo glass arrays. Fluorescence was detected with a GenePix ${ }^{\circledR}$ array reader and the results were analyzed using GenePix pro ${ }^{\circledast}$ and Acuity ${ }^{\circledast}$ software. Results: The expression profile of transcriptional active genes shows a fivefold over or under expression of 78 genes on all arrays. Highly over expressed genes are involved in a variety of immune defense reactions like degrading connective tissue, chemotactic activity on monocytes and inflammatory response. Regulatory genes for cell growth, proliferation, differentiation and apoptosis are more than tenfold increased in all CML patients. Calcium regulating genes, adipose metabolism relevant genes as well as genes with unknown function or hypothetical genes are also regulated. Canditate genes which might play a role in development and progress of CML will be investigated on 800 samples from 160 patients by qPCR. Conclusions: By DNA microarray technology, 78 specific differentially expressed genes could be detected in CML patients. Candidate gene of prognostic interest should be found by further screening of clinical samples of patients treated with various therapies by real-time PCR.

\section{P467}

\section{ALIMTA (Pemetrexed): Correlations between Chemosensitivity pattern and Genexpression}

Eismann U. ${ }^{1}$, Oberschmidt O. ${ }^{1}$, Ehnert M. ${ }^{1}$, Struck S. ${ }^{1}$, Blatter J. ${ }^{2}$, Lahn M. ${ }^{2}$, Ma D. ${ }^{2}$, Niyikiza $C^{2}{ }^{2}$, Paoletti . $^{2}$, Hanauske A.

${ }^{1}$ Allgemeines Krankenhaus St. Georg, Hamburg, Germany,

${ }^{2}$ Eli Lilly \& Company, Indianapolis, USA

Background: The antifolate drug ALIMTA (Pemetrexed, P) is clinically active in several tumor types and has recently been approved for the treatment of pleural mesothelioma. Its structural similarity to folate results in the use of folate pathways for entering the cell such as reduced folate carrier (RFC) and folate receptor- $\alpha$ (FR- $\alpha$ ). Subsequently, folyl polyglutamate synthetase (FPGS) modifies ALIMTA by adding polyglutamate residues. This modification results in decreased drug efflux and increased potency to inhibit the drug's primary target enzymes. $\mathrm{P}$ inhibits enzymes for both purine and pyrimidine neosynthesis including thymidilate synthase (TS), dihydrofolate reductase (DHFR), and glycinamide ribonucleotide formyltransferase (GARFT). The purpose of the present study was to correlate expression of transporter genes of $\mathrm{P}$ with in vitro chemosensitivity of freshly explanted human tumor specimens. Such correlations may help to develop a more rational design for future clinical studies. Methods: Soft-agar cell cloning experiments were performed using freshly biopsied tumor cells (solid tumors, pleural effusions, or ascites). Cells were exposed to several concentrations of $\mathrm{P}$ and clonogenic tumor growth was evaluated after 3 weeks. An aliquot of the same tumo specimens was shock-frozen immediately after removal from the patient Subsequent isolation of total RNA and generation of the respective cDNAs were followed by real-time multiplex PCR experiments using probes for RFC, FR, and GARFT. Results from these gene expression experiments were normalized against $\beta$-actin transcripts. Results: 29 tumor samples were collected. No correlation was observed between colony survival and expression levels of RFC $(\mathrm{R}=0.04 ; \mathrm{n}=18)$ or FR- $\alpha(\mathrm{R}=0.02 ; \mathrm{n}=18)$. However, correlation between GARFT expression and clonogenic survival was noted $(\mathrm{R}=0,7810 ; \mathrm{n}=13$ ). Also, no clear difference was observed in RFC or FR- $\alpha$ gene expression between P-sensitive and P-resistant specimens ( average \pm SD: RFC: $44 \pm 47$ versus $48 \pm 54$; FR- $\alpha$ : $3724 \pm 6596$ versus $2068 \pm 4043$ ) However, a difference in GARFT expression was noted with sensitive specimens presenting lower expression levels compared to resistant specimens $(117 \pm 52$ versus $318 \pm 235)$. These results may indicate a clinical linkage between low GARFT but not RFC or FR- $\alpha$ expression and antitumor activity of P. Supported in part by Eli Lilly \& Company

\section{Poster Session: Proteomics \\ P468 \\ Proteomic Pathway Discovery Reveals that C/EBPalpha \\ Disperses PML Nuclear Bodies to Recruit its own Coactivatorsin a MAPK-Dependent Manner}

Meisel A. *, Kashirin R. *, Christopeit M. *, Hiddemann W., Behre G. Medizinische Klinik III des Klinikums der Universität München Großhadern, Munich, Germany

The tumor suppressor protein $\mathrm{C} / \mathrm{EBPalpha}$ plays a major role in the terminal differentiation of various tissue types, while inactivation of $\mathrm{C} / \mathrm{EBPalpha}$ through different mechanisms is a critical event during tumorigenesis. Downregulation of $\mathrm{C} / \mathrm{EBPalph}$ turned out to be the case in nearly $50 \%$ of all primary lung cancer specimen. In lung cancer cell lines, a total loss of C/EBPalpha expression was reported in even $80 \%$ of the cases. In contrast, restoration of its expression leads to differentiation and ultimately apoptosis. To investigate the mechanisms mediating C/EBPalpha's antiproliferative and differentiation-inducing activity in lung cancer, we performed a proteomewide screen for C/EBPalpha target proteins using 2-D-gel electrophoresis and MALDI-TOF mass spectrometry. Besides 80 other target proteins, we identified PML as a protein being regulated by C/EBPalpha. In fluorescence microscopy studies we could show that the induction of C/EBPalpha leads to a dramatic decrease in the number of PML nuclear bodies. We found out that these changes occur due to a reduction in the slower migrating sumoylated isoforms of PML. In reporter assay, overexpression of PML was increasing the transactivation capacity of C/EBPalpha. CBP could further augment this coactivating effect. Interestingly, Cadmium, which is like C/EBPalpha desumoylating the PML protein and dispersing the PML nuclear bodies, was having the same effect. In earlier studies the MAP-kinases ERK 1/2 and p38 were suspected to be involved in the desumoylation of PML. Consistent with these results C/EBPalpha is also activating ERK and p38. The p38 inhibitor SB203580 could partially abrogate the C/EBPalpha-induced effects on PML nuclear bodies. Taken together, our investigations could identify a mechanism, how C/EBPalpha is targeting PML and CBP to sites of active transcription.

*authors contributed equally

\section{P469 \\ Characterization of Prototypic Lymphoma Cell Lines Reveals Distinct Protein Expression Patterns for Specific Lymphoma Subtypes}

Winkler D., Schneider C., Nitsch D., Döhner H., Stilgenbauer S. Department of Internal Medicine III, University of UIm, Germany

Cell lines are frequently used as model systems to study pathogenic events in malignancies. We analyzed the protein expression of 7 cell lines representative for human lymphoma subtypes by Western blotting: EHEB (B-CLL), JVM-2 (B-PLL), GRANTA-519 (MCL), JURKAT (T-ALL), BL60, NAMALWA, BJAB (all Burkitt lymphoma). Due to their function in apoptosis, cell cycle control and B-cell receptor signaling we focused on the following 24 proteins: p53, RB-1, ATM, GLI, BCL-2, BAX, p21, p27, CDK4, E2F1, CYCLIN-D1/2/3, CIAP2, XIAP, SURVIVIN, SMAC, ARF3, AID, APAF1, AKT1, PI3-K. When comparing the cell lines for the expression of RB-1, ATM, p27, AKT1, APAF1, ARF3, PI3-K, GLI, XIAP, CIAP2, SMAC no significant differences were detected. Compared to the other cell lines, EHEB and the Burkitt lines showed high p53 protein expression. P21 
expression was low in the Burkitt lines. Expression of BCL-2 and BAX, was weak or undetectable in the 3 Burkitt cell lines. Protein levels of the cell cycle regulators CDK4, CYCLIN-D1, CYCLIN-D2 were generally low in the Burkitt cell lines, while high levels of CYCLIN-D3 were observed. The MCL line GRANTA was characterized by higher protein levels of CYCLIN-D1, CDK4 and E2F and lower expression of CYCLIN-D3 when compared to the B-CLL line EHEB. SURVIVIN expression was higher in the Burkitt lines, GRANTA and JURKAT in relation to EHEB and JVM. Strong expression of AID was seen in the Burkitt cell lines BL60 and NAMALWA whereas the other cell lines showed no or weak signals. In conclusion, characteristic protein expression patterns in the cell lines were identified. EHEB (B-CLL) and GRANTA-519 (MCL) could be differentiated by the cell cycle regulators CDK4, CYCLIN-D1/2/3, SURVIVIN and E2F1 indicating enhanced proliferation in GRANTA. No remarkable differences in protein expression were seen between EHEB (B-CLL) and JVM-2 (B-PLL) confirming the pathogenic similarity of both diseases. Remarkably, the characteristic findings in the Burkitt cell lines were reduced levels of the proliferation associated proteins CDK4, CYCLIN-D1, CYCLIN-D2, but a high level of CYCLIN-D3 and of AID in line with a germinal center origin of Burkitt lymphoma. In summary, characteristic protein expression patterns were observed in cell lines derived from distinct lymphoma entities. Therefore, the cell lines appear to reflect the biological behavior of the lymphomas they were obtained from and may provide a basis to elucidate the pathogenesis of different lymphomas.

\section{P470}

\section{Identification of BCR-ABL Dependent Gene Regulation By Using A Combined Proteomics/Phosphoproteomics Approach}

\section{Ziegler P. ${ }^{1}$, Balabanov S. ${ }^{1}$, Hartmann U. ${ }^{1}$, Kammer $W^{2}$,} Nordheim A. ${ }^{2}$, Brümmendorf T.H. ${ }^{1}$

${ }^{1}$ Abteilung Hämatologie/Onkologie, Medizinische Universitätsklinik, Tübingen, Germany, ${ }^{2}$ Abteilung für Molekularbiologie, Interfakultäres Institut für Zellbiologie, Universität Tübingen, Germany

Introduction: The selective tyrosine kinase inhibitor imatinib (formerly STI571, Glivec,) has been shown to block phosphorylation of tyrosine residues by occupying the ATP binding site of the Abl tyrosine kinases as well as platelet-derived growth factor receptor (PDGF) alpha and beta and of the receptor for human stem cell factor (SCF) c-kit. We chose a combined proteomics/phosphoproteomics approach to identify novel downstream targets of imatinib. Material and Methods: Protein expression analysis of K562 leukemic cells was performed using 2-dimensional gelelectrophoresis after treatment with imatinib $(4 \mu \mathrm{M})$ or DMSO for 24 hours and 48 hours. Phosphoproteomics was performed by comparison of large scale phosphotyrosine-immunoprecipitation of imatinib (10 $\mathrm{mM} / 2$ hours) versus DMSO treated K562 cells with subsequent one-dimensional polyacrylamide gelelectrophoresis. Resulting differentially expressed or differentially phosphorylated proteins were analyzed using MALDI-TOF and ESI-MS/MS. Protein identification was performed using Mascot search tool and NCBI nr database. Differential expression or phosphorylation was confirmed by western blot or combined immunoprecipitation and western blot analysis of selected candidate proteins. Results: Unique changes in protein expression levels were observed over a 24 - and 48-hour time course and most of them could be classified based on their known biological function in cell cycle regulation and proliferation control ( e.g. nucleophosmin), focal adhesion and cytoskeletal organization (e.g. vinculin). Two proteins play a role in nuclear import/export (eg. RanBP1), two proteins are involved in amino acid/purine metabolism and the function of two other proteins is still unknown. Phosphoproteomics revealed 8 differentially phosphorylated proteins after two hour treatment of BCR-ABL positive K562 cells, including the recently identified c-cbl an BCR-ABL itself, the latter confirming autophosphorylation of BCR-ABL. The remaining candidates were involved in signal transduction, protein folding or were associated with diverse cellular activities. Discussion: We could detect significant differences in protein expression levels as well as in the phosphorylation pattern of BCR-ABL-dependent cell line K562 upon treatment with imatinib. Ongoing studies are aimed at the analysis of the role of the identified proteins for BCR-ABL induced signal transduction as well as for the development of resistance to imatinib.
P471

Identification and Characterisation of 2-D Protein Spots in Highly Enriched CD34+ Stem Cells From Cord Blood

\author{
Lannert H. ${ }^{1}$, Franz T. ${ }^{1}$, Li X. ${ }^{2}$, Eckstein V. ${ }^{2}$, Lenze A. ${ }^{1}$, Ho A.D. \\ ${ }^{1}$ Department of Internal Medicine $V$, University of Heidelberg, \\ Heidelberg, Germany, ${ }^{2}$ Proteomics Core Facility, EMBL Heidelberg, \\ Heidelberg, Germany
}

Purpose: Proteome analysis is a direct measurement of proteins in terms of their presence and relative abundance in a defined system. The overall aim of a proteomic study is characterization of the complex network of cell regulation. Different states of a cell can be compared and specific qualitative and quantitative protein changes can be identified. We focused our first proteominvestigations on a subset of highly enriched CD34+ stem cells from cord blood (CB). Methods: Mononuclear cells were isolated by a standard FicollHypaque gradient separation method from CB. An Auto-MACS (Miltenyi) and FACS Vantage SE cell sorter (Becton Dickinson) was used to highly enrich ( $>99 \%$ ) CD34+ cells fractions. Sample preparation, determination of protein concentrations, 2D-gel-electrophoresis with a 1D-separation isoelectric focusing (IEF) with Immobilised pH gradient (IPG) strips $(17 \mathrm{~cm}), 2 \mathrm{D}$ separation with SDS-PAGE were performed and described in Proteome Works System (BioRad). Sypro ruby stained gels were used for protein identification by peptide mass fingerprint analysis with matrix assisted laser desorption ionization time of flight mass spectrometry (MALDI-TOF-MS). Results: 1.) The pattern of the CD34+ fraction from CB cells is complex and shows $>1000$ protein spots (dependent on protein concentration) in silver stain using PDQuest.

2.) The most dominant protein spots were characterized by MALDI-TOF analyses. GRP78 and HS7C (working as chaperones) and peptidyl-prolyl cistrans-isomerase (Pin1; involved in cell cycle, transcription) were identified. Conclusions: Proteomics is a highly effective method to describe the genexpression and the cell biology of stem cells on real protein transcription. Highly purified CD34+ cells from CB demonstrate a complex proteom. Our preliminary results show that cell cycle determining chaperones (folding of other protein and responsible for their functional activation or deactivation) and cell cycle controlling proteins like peptidyl-prolyl cis-trans-isomerase are dominantly expressed. Further Proteom-comparison with other subsets of stem cells from different origins (e.g. fetal liver, bone marrow) are currently performed.

\section{P472 \\ Optimization and Standardization of SELDI-TOF MS- Derived Protein Profiles from Hydrophobic ProteinChip ${ }^{\mathrm{TM}}$ Arrays}

Aivado M. ${ }^{1,2,3}$, Spentzos D. ${ }^{1,2,4}$, Alterovitz G. ${ }^{5}$, Grall F. ${ }^{1,2}$, Out H.H. ${ }^{1,4}$, Prall W. ${ }^{1}$, Czibere A. ${ }^{1}$, Cho J.-Y. ${ }^{6}$, Ramoni M.F. ${ }^{5}$, Haas R. $^{3}$, Libermann T.A. ${ }^{1,2,4}$

${ }^{1}$ BIDMC Genomics Center, Beth Israel Deaconess Medical Center and Harvard Medical School, Harvard Institutes of Medicine, Boston, MA, USA, '2Dana-Farber/ Harvard Cancer Center Proteomics Core, Harvard Medical School, Boston, MA, USA ${ }^{3}$ Dept. of Hematology, Oncology and Clinical Immunology, Heinrich Heine-University of Duesseldorf, Germany, ${ }^{4}$ Bioinformatics Core of BIDMC Genomics Center, Harvard Medical School, Boston, MA, USA, ${ }^{5}$ Informatics Program, Children's Hospital and Harvard Medical School, Boston, MA, USA, ${ }^{6}$ Department of Biochemistry, School of Dentistry, Kyungpook National University, Daegu, South Korea

Purpose: Surface-enhanced laser desorption/ionization (SELDI) time-offlight mass spectrometry with ProteinChip ${ }^{\mathrm{TM}}$ arrays (Ciphergen) has facilitated the discovery of disease-specific protein profiles in serum. Such results raise hopes that protein profiles may become a powerful diagnostic tool. To this end, reliable and reproducible protein profiles need to be generated from large sample numbers, accurate mass peak heights are necessary, and the experimental intra- and inter-assay variation of the profiles must be determined. Methods and Results: Therefore, we adapted the entire processing of protein arrays to a fully automated liquid handling system in 192-well format and observed intra-assay coefficients of variation $(\mathrm{CV})$ decreasing from $45.1 \%$ to $27.8 \%$ ( $\mathrm{P}<0.001$ ). Hence, automation not only facilitates highthroughput studies, i.e. up to 24 protein arrays with 16 spots each per day, but also substantially improves the reproducibility of SELDI-TOF-derived profiles. We performed hierarchical cluster analyses on spectra from two 
blinded sample sets ( $\mathrm{n}=14$ each), and investigated the potential usefulness of up to 16 technical replicates. Both analyses demonstrate that 2-4 replicates significantly increase the reliability of the protein profiles. A recent report (Rogers et al., Cancer Research, 2003) on limited long-term reproducibility seemed to concord with our initial inter-assay CVs that varied widely and reached up to $56.7 \%$. However, we discovered that the inter-assay $\mathrm{CV}$ is strongly dependent on the drying time before application of matrix molecule. Therefore, we devised a standardized drying process, and we demonstrate that our optimized SELDI-procedure generates reliable and long-term reproducible protein profiles with CVs ranging from $25.7 \%$ to 32.6 , depending on the SNR-threshold used. Conclusions: In sum, reliable and reproducible serum protein profiles from large-scale studies rely on a high degree of standardization. As we show, this can be accomplished by using an automated liquid handling system, at least 2 replicates, and a uniform drying procedure.

\section{P473}

\section{Proteomics in the Clinical Follow Up of Patients after Allogeneic Hematopoietic Stem Cell Transplantation}

Weissinger E.M. ${ }^{1}$, Kaiser $T^{2}$, Rank $A^{3}{ }^{3}$, Kamal $H{ }^{1}$, Mischak $H^{2.3}$, Kolb H.-J. ${ }^{3}$, Holler $E^{4}{ }^{4}$, Ganser A. ${ }^{1}$, Hertenstein B.

${ }^{1}$ Hämatologie/Hämostaseologie/Onkologie Med. Hochschule

Hannover, Germany, ${ }^{2}$ mosaiques diagnostics $\mathrm{GmbH}$, Hannover,

Germany, ${ }^{3}$ Klinikum Großhadern, Jose-Carreras-Einheit, München,

Germany, ${ }^{4}$ Universitätsklinik Regensburg, Abt. Hämatologie,

Regensburg, Germany, ${ }^{5}$ Abt. Nephroligie, MHH, Hannover, Germany

We have developed a method based on capillary electrophoresis (CE) and mass spectrometry (MS); which allows us to depict and identify all polypeptides in the urine and serum of patients and to compare these to healthy controls. Here we report the application of this method in the screening of 34 patients (25 AML, 3 ALL, 2 CML high risk, 4 other diseases) after alloHSCT. Nineteen patients were transplanted from unrelated donors (18 MUD 1 mismatch), 12 received stem cells from family donors, 3 received haploHSCT and 1 received an autologous transplantation.

GvHD prophylaxis was methotrexat or mycophenolate (MMF) and cyclosporin A in 20 patients and T-cell depletion in 3. After transplantation urine and serum samples were collected twice a week, starting before conditioning until discharge from the ward. Screening of the patients' urine with CE-MS yielded between 700 and 2500 polypeptides defined via their mass, charge, and migration time in the CE-MS. These polypeptides were depicted as a three dimensional picture (contour plot, "Diapat", Fig. 1) and the data for each individual patient were stored in a database, allowing comparison of the different samples of one individual patient as well as comparison within patient groups and controls. Patients with no major complications during the observation time $(n=16)$ gave DiaPats similar to the controls (autologous HSCT, healthy individuals). Eleven patients developing GvHD between day +14 and day +60 showed significant differences with completely new polypeptides appearing and significant changes in concentration of the proteins excreted. Comparison of the DiaPats of all patients with GvHD yielded about 25 polypeptides only present in these patients, while they were never seen in any of the other patient groups. Polypeptides "significant" for GvHD could be detected at least 6 days prior to clinical parameters like skin rash or increase of liver enzymes. Comparison of the "GvHD pattern" to patients with sepsis $(n=5)$, acute renal failure $(n=2)$ or other complications will be discussed. Acute renal failure patients excrete significantly different polypeptides than patients with GvHD. Proteomics may serve as a useful tool for the identification and early diagnosis of complications associated with HSCT

Figure 1: Data generation:

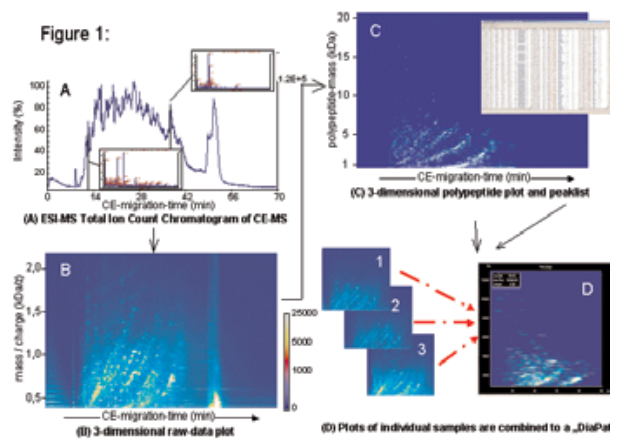

\section{Poster Session: Cytokines}

\section{P474 \\ Functional Activity of in Vivo Primed Granulocytes: A Comparative Study}

\section{Ribeiro D. ${ }^{1}$, Laufs S. ${ }^{1}$, Buss E.C. ${ }^{2}$, Zeller W.J. ${ }^{1}$, Ho A.D. ${ }^{2}$,} Fruehauf $S^{2}$

${ }^{1}$ German Cancer Research Center, Department E0120, Heidelberg, Germany, ${ }^{2}$ University of Heidelberg, Department of Internal Medicine $\checkmark$, Heidelberg, Germany

Recombinant granulocyte colony-stimulating factor (G-CSF) has been widely used in the treatment of chemotherapy-induced neutropenia as well as in mobilisation of peripheral blood stem cells in context with autologous bone marrow transplantation. Beside recombinant G-CSF expressed in E.coli (Filgrastim) and G-CSF expressed in CHO-cells (Lenograstim), pegylated filgrastim (Pegfilgrastim) has been introduced for single-administration into clinical use. Here we study the effects of different G-CSF on functional activity of granulocytes including chemotaxis, oxidative burst and antigen expression as well as potential correlation to G-CSF serum level.

Granulocytes were obtained from patients with hematological malignancies before and after the administration of one of the three G-CSFs and isolated using a polymorphprep density gradient. Chemotactic properties were assessed using a Boyden chamber assay in combination with an under agarose assay, both using fMLP as chemotactic stimulus. Release of superoxide anions served as measure of the oxidative burst after stimulation with PMA using a chemiluminescence assay. The viability and surface antigen expression were assessed by FACS. In addition serum samples have been stored at $-80^{\circ} \mathrm{C}$ for ELISA tests.

FACS analysis showed a decrease in CD10, CD11b and CD62L of the selectin family contrary to an increased expression of the VLA-5 alpha chain CD49, the LPS-receptor CD14 and the IgG receptor Fc $\gamma R I$ (CD64). A stronger effect of lenograstim on $\mathrm{CD} 11 \mathrm{~b}$ and $\mathrm{CD} 14$ could be assessed contrary to filgrastim showing a stronger effect on CD62 and CD64. Further data on pegfilgrastim is to be evaluated.

We observed a decrease of chemotactic activity and non-directed random migration in patients receiving filgrastim, as opposed to the results in patients receiving lenograstim. No obvious differences were found in production of superoxide anions.

Present data shows differences between the commonly used G-CSFs Filgrastim and Lenograstim in granulocyte antigen expression and chemotaxis, further data on patients receiving Pegfilgrastim has to be evaluated.

\section{P475 \\ Differential Effects of Three Tumor Necrosis Factor Antagonists on the Development of LPS Resistance in Human Monocytes}

Kirchner S., Holler E., Haffner S., Andreesen R., Eißner G. Department of Hematology and Oncology, University of Regensburg, Germany

Purpose: Tumor necrosis factor (TNF) is a pleiotropic cytokine critically involved in a number of inflammatory diseases, such as graft-versus-hos disease (GvHD) following allogeneic stem cell transplantation (SCT). However, the versatility of TNF is still not fully understood. Previously, we have shown that the membrane integrated form of TNF (mTNF) can act as a receptor providing resistance of monocytic cells against the proinflammatory stimulus of bacterial endotoxin (LPS), as could be seen by the suppression of LPS-mediated release of soluble apoptosis factors and soluble cytokines, such as TNF and interleukin 1 (IL-1). Reverse signaling of mTNF can be induced by anti-TNF antibodies and soluble TNF receptors that normally antagonize this cytokine in the classical signal direction. In the present report we compared three different TNF reactive agents that are or have been in use in clinical trials for the treatment/prophylaxis of GvHD, i.e. the murine (Fab) fragment $195 \mathrm{~F}$, the humanized monoclonal antibody infliximab and the humanized soluble TNF receptor construct etanercept, respectively. Methods: Apoptosis was assessed by incubation of the micovascular endothelial cell line HMEC with supernatants of LPS-treated monocytes in the presence or absence of the TNF reactive agents for 48 hours. Subsequently, cells were stained with the DNA dye DAPI (4,6-Diamidino-2phenylindole) and subjected to computer-guided fluorescence microscopic 
analysis for nuclear disintegration as typical signs of apoptosis. The detection of soluble TNF and IL-1 was performed by ELISA according to the manufacturers' instructions. Results: Whereas $195 \mathrm{~F}$ and infliximab suppress both, the release of the LPS-induced endothelial cell apoptotic factor and proinflammatory cytokines, etanercept only protected against the LPS-triggered apoptosis activity, but left the LPS-induced cytokine release unchanged. Conclusions: Though it remains to be elucidated whether reverse signaling also occurs in vivo, the data provided could explain a number of clinical observations, including the increased occurrence of fungal infections after infliximab therapy as compared to ethanercept treatment of GvHD. Based on our results we would hypothesize that infliximab completely anergizes monocytes and even blocks desirable anti-inflammatory monocytic responses against secondary infections. Our study strongly suggests to individually re-evaluate cytokine antagonist-based therapeutic approaches in various inflammatory disorders.

\section{P476}

Overexpression of BMP-2 (Bone Morphogenetic Protein 2) in Breast Carcinoma Cells Supports Tumor Formation and Ossification

Clement J.H. ${ }^{1}$, Raida M. ${ }^{2}$, Sänger J. ${ }^{3}$, Bicknell R. ${ }^{4}$, Hortschansky P. ${ }^{5}$, Schmidt A. ${ }^{3}$, Wölfl S. ${ }^{1}$, Harris A.L. ${ }^{4}$, Höffken $K{ }^{1}$

${ }^{1}$ Department of Internal Medicine II and Branch Molecular Biology, Friedrich Schiller University Jena, Germany, ${ }^{2}$ Department of Internal Medicine II, University Clinic Leipzig, Germany, ${ }^{3}$ Institute of Pathology Bad Berka, Germany, ${ }^{4}$ Molecular Oncology Laboratory, The Weatherhall Institute of Molecular Medicine, John Radcliffe Hospital, Imperial Cancer Research Fund, Oxford, UK, ${ }^{5}$ Department for Applied Microbiology, Hans-Knoell-Institute for Natural Product Research, Jena, Germany

Purpose: Bone morphogenetic proteins (BMPs) are expressed in various normal tissues as well as in tumor cells and are involved in the regulation of intracellular processes that support cell survival such as cell cycle control Therefore, we analysed how BMP-2 modulates the behaviour of tumor cells Materials and Methods: An BMP-2 overexpressing cell line (MCF7/BMP2 was created by transfecting MCF-7 with a pcDNA3.1(-) vector containing full-length BMP-2. Cell lines were cultivated with DMEM $+10 \%$ fetal calf serum. BMP-2 mRNA levels were determined by RT-PCR. Protein levels were estimated by western blot and immunohistochemistry. Tumorigenicity assays were performed with MCF7/BMP2 and MCF7/3.1 (for control) using athymic $\mathrm{NuNu}$ mice. Results: Overexpression of BMP-2 resulting in a continuous high level of BMP-2 in the breast cancer cell line MCF-7 (MCF7/BMP2) enhanced the in vitro migratory properties of these cells in comparison to controls (MCF7/BMP2: 15,75 \pm 4,09 SD; MCF7/3.1: 8,42 \pm 4,03 - p=0,004; MCF7: 7,58 $\pm 3,09-\mathrm{p}<0,001 ; \mathrm{n}=12$ ). In a xenograft model without estrogen supplementation MCF7/BMP2 cells formed tumors (9/10), whereas no tumors were found with MCF7/3.1 cells (0/10). MCF7/BMP2 tumors were characterised by the human-specific epithelial membrane antigen (EMA), an increased level of phospho-SMAD1, indicating an elevated activity of the BMP/Smad pathway and a slightly enhanced expression of Ki67. The MCF7/BMP2 cells formed nests surrounded by connective tissue, which contained chondroid and osseous structures. Conclusion: We could demonstrate that elevated levels of BMP-2 enhance the tumorigenic properties of breast carcinoma cells and drive the cells towards a more aggressive phenotype.

This work was supported in part by the Dr. Rainald-Stromeyer-Foundation and the Deutsche Krebshilfe e.V

\section{P477}

\section{Rapid Succession of Stem Cell Mobilisation Cycles in Patients with Chronic Heart Failure - Effects on Haematopoietic and Non-Haematopoietic Organs}

\section{Hüttmann A. ${ }^{1}$, Gutersohn $A .{ }^{2}$, Noppeney $R .{ }^{1}$, Neumann $T^{2}$,} Erbel R. ${ }^{2}$, Dührsen U.'

1Clinics of Hematology and 2Cardiology, Center for Internal Medicine, University Hospital Essen, Germany

Purpose: Circulating (bone marrow deriv ed) stem cells may contribute to the regeneration of non-haematopoietic organs. We subjected patients with chronic heart failure to granulocyte colony-stimulating factor (G-CSF) induced stem cell mobilisation cycles. Herein we report the effects on haematopoietic and non-haematopoietic organs. The impact on cardiac function has been described elsewhere. Methods: 14 male patients with chronic heart failure of New York Heart Association (NYHA) functional class III or IV due to dilated or ischemic cardiomyopathy were subjected to four G-CSF treatment periods of 10 days' duration separated by three 10-day treatmentfree intervals followed by monthly control visits until day 180 after the start of therapy. The dose of G-CSF (starting dose: $480 \mu \mathrm{g} \mathrm{sc}$ bid) was adjusted on a daily basis to reach a leukocyte count of 45.000 per $\mu$ l by day 4 and maintain a level of $45.000-50.000$ per $\mu 1$ until day 10 . Analyses included physical examination, ultrasonography of the spleen and laboratory investigations Results: In the haematopoietic system, G-CSF induced a rapid increase in cells of all leukocyte lineages with return to levels equal to (granulocytes) or lower than those before treatment (monocytes, lymphocytes) during the treatment-free intervals. Red cells remained unchanged, and platelets decreased followed by rebound thrombocytosis. Increase in $\mathrm{CD} 34^{+}$cells was highly variable with low values in patients receiving amiodarone. For each patient, the changes induced were identical through all cycles, but the G-CSF dose in the first cycle was significantly higher than in subsequent cycles. Between patients, an inverse correlation was observed between the leukocyte level reached and the dose of G-CSF administered. Serum levels of bilirubin decreased, while creatinine and urea nitrogen increased. Conclusions: Sequential stem cell mobilisation is feasible and well-tolerated, inducing identical alterations in all treatment cycles. G-CSF responsiveness varies among patients and is increased by pre-treatment with G-CSF. Notably, repetitive $\mathrm{CD}_{3} 4^{+}$cell mobilisation in 10-day intervals is feasible in the absence of leukapheresis, indicating that the size of the stem cell pool is maintained despite excessive G-CSF stimulation.

\section{Poster Session: Gene Therapy}

\section{P478}

\section{The Formation of Hybrid TCRs can be Averted by the} Single Chain TCR Concept in Adoptive Immunotherapy

\section{Voss R.-H. ${ }^{1}$, Thomas S. ${ }^{1}$, Kuball J. ${ }^{1}$, Engel R. ${ }^{1}$, Guillaume P. ${ }^{2}$, Romero . $^{2}$, Huber $C .{ }^{1}$, Theobald $M$.}

${ }^{1}$ Department of Hematology and Oncology, Johannes GutenbergUniversity, Mainz, Germany, ${ }^{2}$ Ludwig Institute for Cancer Research, University of Lausanne, Lausanne / Epalinges, Switzerland

Purpose: The T-cell receptor (TCR) is able to recognize human tumour associated antigens (TAA) and subsequently triggers a complex signaling cascade that results in the initiation of cytotoxic effector functions in $\mathrm{CD}^{+} \mathrm{T}$-cells. In human gene therapy, high-affinity TCRs have been retrovirally introduced into T cells to eradicate tumour cells in vivo. In order to circumvent self-tolerance to human TAA, high-avidity $\mathrm{T}$ cells were generated in various HLAA2.1 transgenic mouse models to induce HLA-A2.1-restricted immune responses directed against human TAA, such as peptides originating from p53 and MDM2. A2.1-transgenic mice served as a tool to create CD8-independent p53-specific high-affinity TCRs as compared to HuCD8xA2 $\mathrm{K}^{\mathrm{b}}$ transgenic mice that resulted in CD8-dependent MDM2-specific TCRs These TCRs were ideally suited for studying the contribution of the coreceptor CD8 on cytolytic efficacy. Methods: A major concern was to determine as to whether or not murine TCRs are able to form hybrid TCRs with the endogenous ones potentially raising the issue of autoimmunity. Amino acid substitutions that affect interchain affinity will have an impact on surface expression, the formation of hybrid TCRs and functionality in terms of cytokine secretion and cytolysis in chromium release assays. A multitude of TCR constructs, designed as either murine and partially humanized double chain TCRs (dcTCR) or as single chain TCRs (scTCR), have been assayed on their functional outcomes and consequences for the endogenous TCRs. Single chain TCR constructs have been designed by covalently hooking up the variable $\mathrm{V} \alpha$ and $\mathrm{V} \beta$ domains as major determinants for antigen recognition via a linker to the invariant $C \beta$ domain for membrane anchoring. This strategy enables the irreversible linkage of the variable domains in order to abrogate potential pairing with endogenous TCR chains. Results: The expression of single murine TCR chains documents their capability to form hybrid TCRs a tendency that can be further increased by the humanization of the constant domains. Point mutations that impair pairing as deduced from protein structure reduce their surface expression and inversly accumulate in the cytoplasma. Although scTCRs were expressed on the surface of T-cells, they were non-functional in terms of cytotoxicity against $\mathrm{T} 2$ cells pulsed with either p53.264-272 or MDM2.81-88 peptides. To supplement the missing Co 
domain, a truncated TCR $\alpha$ was generated comprising the $\mathrm{C} \alpha$ domain preceded by the TCR $\alpha$ signal peptide. This construct was coexpressed with either the p53- or the MDM2-specific scTCR to provide them with the missing domain that is believed to harbor a binding site for CD8, the $\alpha$-CPM consensus motif, FETDxNLN. We found that both scTCRs of different CD8-dependency require the $\mathrm{C} \alpha$ domain for function at all while only the $\mathrm{CD} 8$-independent p53-specific scTCR gave rise to substantial cytotoxicity comparable to that of wildtypic double chain TCR even for an $\alpha$-CPM-silenced $\mathrm{C} \alpha$-construct. Furthermore exclusively the CD8 independent scTCR/C $\alpha$ construct provided sufficient structural avidity to stain both $\mathrm{CD}^{+}$as well as $\mathrm{CD}^{+}{ }^{+} \mathrm{T}$ cells in tetramer analysis. Formation of the mandatory disulfide bridge linkage has been proven for them by western blotting. Conclusions: We do have clear evidences that the scTCR concept is comparable to wildtypic TCRs in terms of functional avidity despite some impairment in structural avidity making them an amenable tool in adoptive cancer therapy.

\section{P479}

\section{Function of HSV-TK Suicide Gene Modified Canine T Lymphocytes}

Weber M. ${ }^{1}$, Günther W. ${ }^{1}$, Weissinger E. ${ }^{2}$, Kolb H.J. ${ }^{1,3}$

${ }^{1}$ Clin. Coop. Group Hematopoietic Cell Transplantation, GSF -

National Research Center, Munich, Germany, ${ }^{2}$ Dept. of

Hematology/Oncology, Medical School Hannover, Germany,

${ }^{3}$ Dept. Med III, Klinikum Großhadern, University of Munich, Germany

Purpose: Adoptive immunotherapy with donor lymphocyte infusion after stem cell transplantation is the treatment of choice for a number of haematological disorders. The transfer of donor T cells is limited by the occurrence of graft versus host disease (GvHD). A promising way to control GvHD is the use of a suicide gene. Here we describe to production, analysis and transfusion of canine T cells transduced with the HSV-Tk suicide gene. Methods: Transduction was performed using virus supernatant generated with the PG13 packaging cell line. After $72 \mathrm{~h}$ stimulation with $5 \mu \mathrm{g} / \mathrm{ml}$ PHA-M and 100 $\mathrm{U} / \mathrm{ml}$ IL-2 transduction of $\mathrm{T}$ cells was performed by spin-inoculation on fibronectin coated-plates. On day 8 the transduced $\mathrm{T}$ cells were purified with immunomagnetic beads against the LNGFR marker gene. More than $95 \%$ of the cells were positive for LNGFR expression. Cells were susceptible to the in vitro treatment with ganciclovir (GCV) in therapeutic concentrations. Alloreactivity of gene modified cells was comparable to non-transduced $T$ cells. Results: In vivo results were obtained in a DLA-identical setting. The animals were transplanted with T-cell depleted bone marrow from a DLAidentical littermate. 60-80 days after BMT gene modified T cells were infused into the host. The functionality of these cells was assayed by the conversion of haematological chimerism. Gene modified cells were also capable of transferring immunity to a foreign antigen from the donor to the host. The persistence of gene modified cells in the blood was shown by FACS and PCR. The in vivo ablation of gene modified cells by 4 doses of GCV $(10 \mathrm{mg} / \mathrm{kg} / \mathrm{d})$ was shown by FACS and PCR.

We generated gene modified CTLs against DLA and tested the cells in vitro. The capacity of gene modified CTLs to suppress the growth of PHA-blasts was analyzed in a Delta-Assay. The results showed a highly specific suppression of the growth of PHA-blasts incubated with gene modified CTLs. At an $\mathrm{E} / \mathrm{T}$ ratio of 1.25 to 1 we measured a growth suppression of $96.7 \%( \pm 2.88 \%)$ compared to the control. CTLs not transduced with the HSV-TK suicide gene but otherwise treated in the same way as transduced cells resulted in a growth suppression of $97.3 \%( \pm 0.48 \%)$. Conclusions: We have shown the transduction and adoptive transfer of canine $\mathrm{T}$ cells and antigen-specific $\mathrm{T}$ cells and showed their function in vitro and in vivo. On the basis of these results will be develop an experimental canine GvHD model with gene modified T cells.

\section{P480}

\section{Unmodified Oligodeoxynucleotides Require Single- Strandedness to Induce Targeted Repair of a Chromosomal EGFP Gene}

\section{Radecke F., Radecke S., Schwarz K.}

Institute for Clinical Transfusion Medicine and Immunogenetics UIm $\mathrm{GmbH}$; Department of Transfusion Medicine, University Hospital UIm, Germany

Purpose: A number of genetic defects in humans are due to point mutations in a single, often tightly regulated gene. Genetic treatment of such defects is preferably done by correcting only the altered base pair at the endogenous locus rather than by a gene replacement strategy involving viral vectors. Promisingly high repair rates have been achieved in some systems with the non-viral approach of transfecting chimeric RNA/DNA oligonucleotides (chimeraplasts). However, since this technique does not yet perform robustly, several parameters thought to be important in oligonucleotide-mediated gene repair were examined. Methods: A series of transgenic HEK-293 cell clones has been established harbouring high or low copy numbers of a point-mutated 'Enhanced Green Fluorescent Protein' (EGFP) gene as the target. At the level of single living cells, repair efficiencies were measured by FACS regarding topology (single-stranded, double-stranded), exonuclease protection (four phosphorothioate linkages at both ends), polarity (sense, antisense), and length (13mer, 19mer, 35mer, 69mer) of the oligonucleotide. Results: When targeting chromosomal loci, up to $0.2 \%$ corrected cells were obtained with single-stranded unmodified oligodeoxynucleotides, whereas a chimeraplast, its DNA analogue, and double-stranded DNA fragments were practically non-functional. Correction efficiencies correlated with target gene copy numbers. Modifying exonuclease resistance, polarity or length of singlestranded oligodeoxynucleotides did not enhance repair efficacy above the sub-percentage range. Conclusions: Successful chromosomal reporter gene repair in HEK-293 cells required an oligodeoxynucleotide to be singlestranded. In concert with the gene copy number correlation, functional interaction between the repair molecule and the target site seems to be one bottleneck in targeted gene repair.

\section{P481 \\ Tumor Cells Escape Suicide Gene Therapy by Genetic and Epigenetic Instability}

Frank O. ${ }^{1,2}$, Rudolph C. ${ }^{3}$, Heberlein $C^{4}{ }^{4}$, von Neuhoff $N^{3}$, Schröck E. ${ }^{5}$, Schlegelberger $B{ }^{3}{ }^{3}$, Fehse $B{ }^{6}{ }^{6}$, Ostertag $W .{ }^{1}$, Stocking C. ${ }^{1}$, Baum C. ${ }^{1,7,8}$

${ }^{1}$ Department of Cell \& Virus Genetics, Heinrich-Pette-Institute, Hamburg, Germany, ${ }^{2}$ current address: Medical Clinic III, Faculty of Clinical Medicine Mannheim, University of Heidelberg, Mannheim, Germany, ${ }^{3}$ Institute for Cell and Molecular Pathology, Hannover Medical School, Hannover, Germany, ${ }^{4}$ Celltec Biotechnology $\mathrm{GmbH}$, Hamburg, Germany, ${ }^{5}$ Institute of Clinical Genetics, Medical Faculty Carl Gustav Carus, Dresden, Germany, ${ }^{6}$ Bone Marrow Transplantation, University Hospital Eppendorf, Hamburg, Germany,

${ }^{7}$ Division of Experimental Hematology, Cincinnati Children's Hospital Medical Center, Cincinnati, Ohio, USA, ${ }^{8}$ Department of Hematology and Oncology, Hannover Medical School, Hannover, Germany

Purpose: Transfer and expression of suicide genes is one cornerstone of cancer gene therapy and is also considered as a proactive tool to enhance the safety of somatic transgenesis. Using viral or physicochemical gene transfer procedures, numerous preclinical and some clinical trials have been conducted, identifying insufficient gene delivery as a major limitation. We addressed whether retrovirus-mediated suicide gene therapy would result in a predictable anti-tumor efficiency, given that problems related to gene transfer are solved or that the suicide gene is used in a proactive approach. Methods: We induced an experimental tumor by transplanting retrovirally engineered murine EL-4 lymphoma cells in congeneic C57B1/6 mice. The vectors encoded the thymidine kinase (TK) gene of herpes simplex virus and the neomycin resistance gene. Results: Systemic administration of the prodrug ganciclovir (GCV) resulted in reversal of transduced clonal and polyclonal tumors in vivo. However, both uncloned and cloned EL-4 tumors gave rise to GCV-resistant subclones, eventually causing tumor relapse. GCV-resistant tumors showed postinsertional alterations of transgene structure, or loss of the entire transgene. A complete loss of a fusion chromosome that contained the retroviral suicide gene was confirmed by spectral karyotyping (SKY analysis). Transgene silencing occurred in another EL-4 tumor clone. Conclusions: We conclude that genetic as well as epigenetic instability related to biological features of the tumor, the insertion site and the gene transfer system limit the efficiency of retroviral suicide gene therapy. 
P482

\section{Additive Effects in the Induction of Apoptosis in Leukemic Cells by Wt1 and Bcr-Abl Specific siRNAs}

\author{
Elmaagacli A.H. ${ }^{1}$, Koldehoff M. ${ }^{1}$, Peceny R. ${ }^{1}$, Klein-Hitpass $L .{ }^{2}$, \\ Ottinger H. ${ }^{1}$, Beelen D.W. ${ }^{1}$ \\ ${ }^{1}$ Department of Bone Marrow Transplantation, University Hospital of \\ Essen, Germany, ${ }^{2}$ Institute for Cell Biology, University Hospital \\ Essen, Germany
}

We studied the effect of siRNA targeting wt1 in leukemic cells and normal CD34- positive cells with regard to proliferation, induction of apoptosis, and cell differentiation. Furthermore, we evaluated if the additional use of bcr-abl siRNA can augment the antileukemic effects of wt 1 siRNA in CML cells. A reduction of wt1 gene expression measured by real-time RT-PCR was observed in all studied cell lines: K-562, Kasumi-1, MV 4-11 and NB-4, as well as in cells of AML- and CML patients. We found a two-fold increase of induced apoptosis in MV4-11 cells, NB-4 cells and Kasumi- 1 cells $(\mathrm{p}<0.01)$ and a moderate increase in K-562 after transfection with wt1 siRNA versus controls $(\mathrm{p}<0.02)$. Proliferation was strongly inhibited in all studied leukemic cell lines and leukemic cells of patients by wt1 siRNA. In normal CD34-positive cells, the proliferation was only slightly inhibited by about $20 \%$ and no induction of apoptosis was found.

Transfection with both siRNAs together inhibited the proliferation rate additionally compared to transfection with bcr-abl siRNA or wt1 siRNA alone $(\mathrm{p}<0.01)$ in the K-562 cell line and CML cells. The rate of induced apoptosis was more announced than transfection with each siRNA alone $(\mathrm{p}<0.01)$.

We performed a microarray analysis and found that mostly genes involved with DNA synthesis or metabolism, transcriptional regulation, cell signaling and small molecule metabolism, were regulated by the silencing of wt 1 gene in K562 cells

WT1 specific siRNAs had no effect on expression of surface differentiation markers. Wt1 seems to be an interesting target in leukemic cells for new therapeutic strategies.

\section{P483}

\section{Efficient Stimulation of Virus-Specific CD4 ${ }^{+}$and $C D 8^{+}$ T-Cells by T-Cells Modified to Express a Viral Candidate Antigen}

\author{
Adamopoulou E.C. ${ }^{1}$, Hauschulz K. ${ }^{1}$, Kuntz G. ${ }^{1}$, Neff C.1, \\ Einsele H. ${ }^{1}$, Rammensee H.G. ${ }^{2}$, Topp M.S. \\ ${ }^{1}$ Department of Hematology and Oncology and, ${ }^{2}$ Department of \\ Immunologie, Universitätsklinikum Tübingen, Germany
}

Adoptive transfer of T-cells expressing the herpes simplex thymidine kinase induces an strong immune response against the transgene in HIV patients and in allogeneic SCT recipients resulting in eradication of transferred cells. The elimination is mediated by a vigorous T-cell response against the viral thymidine kinase protein. Therefore we reasoned that human T-cells if presenting a viral antigen might represent a novel class of antigen presenting cells. Human T-cells were retrovirally modified with the viral model antigen CMVpp65 creating T- $\mathrm{APC}_{\mathrm{CMV} \text { pp } 55}$ that in turn were used to stimulated a CMV-specific Tcell response. In the first set of experiments $\mathrm{T}-\mathrm{APC}_{\mathrm{CMVpp} 65}$ could activated CMVpp65 T-cell clones as efficiently as CD40L-activated B-cells modified to express $\mathrm{CMVpp65}$ (B-APC ${ }_{\mathrm{CMVpp65}}$ ). In addition if autologous fresh PBMC were used as effectors population both $\mathrm{T}-\mathrm{APC}_{\mathrm{CMVpp} 65}$ and $\mathrm{B}-\mathrm{APC}_{\mathrm{CMVpp} 65}$ could stimulated a combined $\mathrm{CD} 4^{+}$and $\mathrm{CD} 8^{+} \mathrm{CMV}$-specific T-cell responses from CMV-seropositive donors. In vitro stimulation for 8 days with both $\mathrm{T}$ $\mathrm{APC}_{\mathrm{CMV} p \text { p } 65}$ and $\mathrm{B}-\mathrm{APC}_{\mathrm{CMV} p 65}$ resulted on average in a 80 -fold expansion of virus-specific T-cells, which in turn could kill efficiently both $\mathrm{CMV}_{\mathrm{pp} 65}$ modified LCL's as well as CMV-infected fibroblasts. To address the question why $\mathrm{T}-\mathrm{APC}_{\mathrm{CMV} p 65}$ can stimulated such a vigorous T-cell response, the costimulatory ligands $\mathrm{CD} 70, \mathrm{CD} 80, \mathrm{CD} 86,4 \mathrm{BB}-\mathrm{L}$ and $\mathrm{CD} 40 \mathrm{~L}$ on T- $\mathrm{APC}_{\mathrm{CMVpp} 65}$ were evaluated and compared to $\mathrm{CD} 40 \mathrm{~L}$-activated B-cells. All costimulatory ligands were expressed by $\mathrm{T}_{-} \mathrm{APC}_{\mathrm{CMVpp} 65}$ but a lower level than $\mathrm{B}$ $\mathrm{APC}_{\mathrm{CMVpp} 65}$. To determine, if lower expression of costimulatory ligands in $\mathrm{T}$ $\mathrm{APC}_{\mathrm{CMVpp65}}$ resulted in a different phenotype of generated CMV-specific Tcell lines, the expression of corresponding costimulatory receptors and homing molecules were compared. Both generated T-cell lines displayed a similar mixed population of peripheral memory and effectors antigen-specific T-cells with same percentage of central memory T-cells $(<25 \%)$ based on expression of the chemokine receptor CCR-7.
In summary, T-APC modified to express viral antigen can induce a strong combined $\mathrm{CD}^{+}$and $\mathrm{CD}^{+}$viral $\mathrm{T}$-cell response. T-cell lines do not differ in expansion and phenotype from virus-specific T-cells generated by professional antigen presenting such as CD40L-B-cells. Adoptive transfer of TAPC modified to express viral antigens may therefore present a new potential vaccination strategy for inducing viral immunity in the immunocomprimised host.

\section{P484}

\section{Oncolytic Adenoviruses for Acute Myeloid Leukemia}

\section{Brandts C., Gallan M., Berdel W.E., Serve H.}

Department of Medicine, Hematology/Oncology and Institute of Pathology, University of Münster, Germany

Tumor-selective replicating viruses are a promising new approach for the treatment of human cancer, as they may offer appealing advantages over conventional cancer therapy. While chemotherapeutic drugs distribute more or less evenly in the human body and follow log kinetics of cell killing, local replication of administered oncolytic viruses amplifies the input dose and creates a high concentration of a therapeutic agent at the target site. This may provide increased potency, while limiting side effects. Although intravenous virus delivery for metastatic or systemic disease has been found to be safe, several major hurdles remain unsolved.

Among the adenovirus serotypes, group C viruses (particularly serotypes 2 and 5) have been extensively studied. Their attachment to the cell is mediated by the coxsackie-adenovirus-receptor (CAR), while virus endocytosis is dependent on $\mathrm{a}_{\mathrm{v}}$ integrins. However, many advanced tumors loose CAR expression, preventing efficient adenovirus attachment and infection. On the contrary, group B adenoviruses have recently been shown to attach and infect cells in a CD46-dependent manner, independently of CAR. We chose to examine the natural tropism of group B adenoviruses for CAR-negative malignancies. Here, we demonstrated that myeloid leukemia cell lines as well as primary AML blasts expressed little or no CAR on their cell surface. However, we observed strong expression of CD46 (and $a_{v} b_{3}$ integrins) on the surface of myeloid cell lines and primary AML blasts. Furthermore, myeloid leukemia cells were readily infected by adenovirus serotype 35 co-expressing GFP (a group B adenovirus) at multiplicities of infection of 10 and 50, while serotype 5 (group C) was not. The susceptibility of myeloid leukemia cells to group B adenoviruses were confirmed by immunofluorescence for a panel of wildtype group B serotypes. We studied CD46-expression in myeloid differentiation, and observed marked changes in overall expression, as well as splice variants, in the majority of cell systems examined. Importantly, myeloid leukemia cells supported efficient viral DNA synthesis of group B adenoviruses and efficient viral replication was observed in the majority of cell lines examined.

We conclude that the use of group B adenoviruses may be useful for designing oncolytic adenoviruses for CAR-negative tumors, such as myeloid leukemia. Furthermore, primary AML blasts may be a useful model to test viral infection and replication in primary tumor cells, circumventing the limitations of xenograft animal models for human viruses. Current efforts concentrate on achieving tumor-selective replication and several strategies will be discussed.

\section{P485 \\ Enhanced Tumor Cell-Specific Expression of Nbk/Bik by Combining the CEA Promoter and the Tet off System in An Adenoviral Vector}

\section{Gillissen B., Forro G., Hemmati P., Dörken B., Daniel P.T.}

Dept. of Hematology, Oncology and Tumor Immunology, University Medical Center Charité, Humboldt University, Berlin.

Adenovirus vectors are considered as good gene delivery vectors because they achieve transient high-level transgene expression and high gene transfer efficiency. Recombinant adenovirus vectors carrying proapoptotic genes are a promising approach in the therapy of human malignant tumors. To investigate the antitumor activity of the proapoptotic BH3-only protein Nbk/Bik, we constructed a single recombinant adenoviral vector for the regulated $\mathrm{Nbk} / \mathrm{Bik}$ expression based on the Tet off system. The E1 and E3 region of the adenovirus were replaced by expression cassettes for $\mathrm{Nbk} / \mathrm{Bik}$ and for the reverse tetracycline regulated transactivator (tTA), respectively. Whereas Nbk/Bik was under control of the tetracycline responsive element, a constitutive CMV 
promoter drove the tetracycline-regulated transactivator. Thus, addition or withdrawal of tetracycline can regulate Ad-Nbk expression. Ad-Nbk expression induced cell death in a variety of human cancer cell lines examined including breast cancer, colon cancer, liver cancer, neuroblastoma and osteosacroma cells, indicating a possible role of $\mathrm{Nbk} / \mathrm{Bik}$ as a therapeutic gene in cancer gene therapy. Nevertheless, in this CMV driven expression system expression of $\mathrm{Nbk} / \mathrm{Bik}$ is not limited to cancer cells. To restrict expression of suicide genes to cancer cells, tissue and tumor-specific promoters have been widely used. However the expression levels of these promoters are generally low.

Carcinoembryonic antigen (CEA) is expressed by many tumors of the colorectal origin, and it is a clinically useful a parameter for colorectal carcinoma recurrence. To achieve tumor specific and regulated expression of $\mathrm{Nbk} / \mathrm{Bik}$ and to enhance CEA driven expression of Nbk/Bik we replaced the $\mathrm{CMV}$ promoter in the recombinant adenovirus by the CEA promoter. In this vector system the CEA promoter is driving the expression of the tTA which then can activate transcription of $\mathrm{Nbk} / \mathrm{Bik}$ from the tetracycline responsive element. In contrast to the CMV vector system, transduction of cells with the CEA adenovirus vector induces Nbk/Bik expression and cell death only in CEA positive cells. Thus, this vector is a model for targeting suicide gene expression to tumor cells and can be adapted to other human tumors in conjugation with different cell type and tumor-specific promoters.

\section{P486}

\section{Real-Time Quantitative PCR-Based Method for Rapid Titration of Adeno-Associated Virus Serotypes 1 - 6}

Stiefelhagen M. ${ }^{1}$, Veldwijk M.R. ${ }^{1,2}$, Laufs S. ${ }^{1}$, Kleinschmidt J.A. ${ }^{3}$, Wenz F. ${ }^{2}$, Zeller W.J. ${ }^{1}$, Fruehauf $S{ }^{4}{ }^{4}$

${ }^{1}$ Dept. E120, German Cancer Research Center, INF 280, Heidelberg, Germany, ${ }^{2}$ Department of Radiation Oncology, Universitätsklinikum Mannheim, University of Heidelberg, Mannheim, Germany,

${ }^{3}$ Dept. F010, German Cancer Research Center, Heidelberg, Germany, ${ }^{4}$ Department of Internal Medicine V, University of Heidelberg,

Heidelberg, Germany

Adeno-associated virus (AAV) vectors are a promising model for future approaches in gene therapies. Recombinant AAV-2 (rAAV-2) vectors are currently used in clinical gene therapy studies for hemophilia, cystic fibrosis and preclinical studies. Still, some cell types and tissues do not seem to be susceptible to rAAV-2 vectors. Here, recombinant vectors based on other AAV serotypes could help to overcome this limitation.

Using a two plasmid production system established by Grimm and colleagues (Mol. Ther.,7, 2003), recombinant vector stocks of the AAV serotypes 1- 6 can now readily be produced. Since the differences in susceptibility of the reference cell lines to the serotypes used for titration do not allow comparison between titers, a cell-free assay is imperative.

Previously, we established a highly standardized cell-free and high throughput titration assay with low variability $(\mathrm{C} . \mathrm{V} .<0.10)$, based on the quantitative PCR (Veldwijk et al, Mol .Ther., 6, 2002). Now, the assay has been modified to allow titration of rAAV stocks based on serotypes 1-6. For this purpose, the primers and probe were placed between the poly-A and 3 ITR present in all our rAAV vectors. The rAAV-2 plasmid pTR-UF5 was used as standard, thereby allowing absolute quantification.

The highly standardized titration by RQ-PCR described here is our application of choice for rAAV serotype titration, as it allows the determination of the amount of vector particles for any serotype, where differences in susceptibility of the reference cell lines used in the functional titration assay formerly set the limit.

\section{P487}

\section{Small Interfering RNAs Directed against Growth Factor Independence 1B Gene (GFI-1B) Inhibit Proliferation and Induce Apoptosis in Leukemic Cell Lines}

\section{Koldehoff M. ${ }^{1}$, Steckel N.K. ${ }^{1}$, Trenschel R. ${ }^{1}$, Beelen D.W. ${ }^{1}$ Opalka B. ${ }^{2}$, Elmaagacli A.H. ${ }^{1}$ \\ ${ }^{1}$ Department of Bone Marrow Transplantation, University Hospital of Essen, Germany, ${ }^{2}$ Department of Internal Medicine (Tumor Research), University Hospital of Essen, Germany}

Gfi-1B is a zinc finger protein which is expressed exclusively in hematopoietic cells. Gfi-1B regulates transcription during erythropoiesis and is also involved in regulating the process of hematopoietic cell differentiation and megakaryopoiesis. We studied the effect of transfection with small interfering RNA (siRNA) targeting Gfi-1B in leukemic cells and normal CD34- positive cells in regard of proliferation, inducing apoptosis, and cell differentiation. Further, we evaluated if the post-transcriptional gene silencing of Gfi-1B mRNA can be augmented by use of two further siRNAs targeting the bcr-abl hybridgene or targeting the wt-1 gene expression in the cell line K-562. A reduction of Gfi-1B gene expression measured by real-time RT-PCR to amounts between $12 \%$ and $45.4 \%$ (mean) was observed in the K562 and Hel cell lines compared to controls ( controls were set up to $100 \%$ ). We found a two-fold increase of induced apoptosis in MV4-11 cells, NB4 cells and a four-fold increase of apoptosis in Hel, Kasumi-1, K-562 cell lines 24 hours after transfection with Gfi-1B siRNA versus controls. Proliferation was strongly inhibited (of about $80 \%$ ) in cell lines: Hel, Kasumi-1, MV4-11 by Gfi-1B siRNA and moderate decreased (of about $\geq 50 \%$ ) in NB4 and K-562 leukemic cells. In normal CD34-positive cells, the proliferation was inhibited too (by about $70 \%$ ). In contrast to leukemic cells, no induced apoptosis was found in CD34+ cells after Gfi-1B siRNA transfection. Cotransfection with Gfi-1B siRNA, wt-1 siRNA, and bcr-abl siRNA did not inhibited the proliferation rate more effectively than transfection with Gfi-1B siRNA alone. The rate of induced apoptosis was constant to the transfection with each siRNA. No synergistic effects of Gfi-1B siRNA with bcr-abl siRNA or wt1 siRNA was measured. The transfection of Gfi-1B siRNA in CD34+ cells had no influence on differentiation markers CD13, CD14, CD33, CD34, CD45, CD64 and glycophorin A. Glycophorin A expression increased after transfection with Gfi-1B siRNA in the Hel cell line, whereas CD64 positive monocytes increased in the K-562 cell line after the transfection with Gfi-1B siRNA. These findings suggest that Gfi-1B seems to be a promising target for new therapeutic strategies with siRNAs in the treatment of erythroleukemic cells.

\section{P488 \\ Efficient Transfer of siRNAs and Plasmids in CD34 ${ }^{+}$ Hematopoietic Stem and Progenitor Cells using Nucleofection}

\section{Graef $T^{1}$, Baer A. ${ }^{1}$, Neumann F. ${ }^{1}$, Steidl U. ${ }^{2}$, Kobbe $G .{ }^{1}$,} Rohr U.-P. ${ }^{1}$, Haas R. ${ }^{1}$, Kronenwett R.

${ }^{1}$ Dept. of Hematology, Oncology and Clinical Immunology, University of Düsseldorf, Germany, ${ }^{2}$ Harvard Institutes of Medicine,

Hematology/Oncology Division, Boston, MA, USA

Gene silencing using small interfering RNAs (siRNAs) has become a powerful method for studying gene function. The use of siRNAs in human primary cells continues to accelerate applications such as target validation and therapeutic approaches that could lead to new gene-specific siRNA-based therapeutics. However, previously leukaemic cells and CD $34^{+}$haematopoietic stem and progenitor cells have been demonstrated to be resistant to most of non-viral gene transfer methods, which has limited their use for siRNA experiments. The aim of this study was to evaluate a new method for transfection of primary CD34 ${ }^{+}$cells and leukaemia cell lines and to improve the efficiency of siRNA delivery. We used a novel electroporation based technique called nucleofection. This novel technique uses a combination of special electrical parameters and specific solutions to deliver siRNA directly to the cell nucleus under mild conditions

$1-2 \times 10^{6} \mathrm{CD}^{2} 4^{+}$haematopoietic stem and progenitor cells immunomagnetically enriched from peripheral blood mononuclear cells and the human chronic myeloid leukemia cell line K562 were nucleofected using the human $\mathrm{CD}^{+} 4^{+}$cell Nucleofector ${ }^{\mathrm{TM}} \mathrm{Kit}$ and the cell line Nucleofector ${ }^{\mathrm{TM}} \mathrm{KitV}$. The cells were nucleofected with $10 \mu \mathrm{g}$ non-silencing rhodamine-labelled control siRNA or with $2.5 \mu \mathrm{g}$ plasmid pmaxGFP encoding for the green fluorescent protein (GFP). At different time points post nucleofection the cells were analyzed by flow cytometry and fluorescence microscopy. Dead cells and CD34 cells were excluded by propidium iodide (PI) staining and gating. We obtained a transfection efficiency in $\mathrm{CD} 4^{+}$cells of $84,4 \%$ for rhodaminelabelled siRNAs and of $32 \%$ for pmaxGFP as assessed $4 \mathrm{~h}$ following nucleofection. PI staining $48 \mathrm{~h}$ post nucleofection showed that $19 \%$ of cells were dead. In K562 transfection efficiency was 58\% for rhodamine-labelled siRNAs and $86 \%$ for pmaxGFP as assessed $3 \mathrm{~h}$ and $12 \mathrm{~h}$ following nucleofection. In K562 a greater toxicity was found in comparison with $\mathrm{CD} 34^{+}$cells as $26.9 \%+/-11.3 \%$ of cells were dead $48 \mathrm{~h}$ post nucleofection.

In conclusion, nucleofection is a useful method for delivering of siRNAs and plasmids into primary $\mathrm{CD}^{+} 4^{+}$hematopoietic stem and progenitor cells. The study provides the basis for knock-down experiments for target validation using siRNAs. 
P489

\section{Genetic Modification Of Human Cord Blood (UCB) Cells Repopulating Non-Obese Diabetic/Severe Combined Immuno Deficiency (NOD/SCID) Mice}

\section{Ebeling P. ${ }^{1}$, Bach P. ${ }^{1}$, Sorg U.R. ${ }^{1}$, Trarbach $T .{ }^{1}$, Hanenberg $H^{2}{ }^{2}$} Seeber S. ${ }^{1}$, Flasshove M. ${ }^{1}$, Moritz T.

'Dept. of Internal Medicine (Cancer Research). University of

Duisburg-Essen Medical School. Essen. Germany, ${ }^{2}$ Clinic for

Paediatric Haematology and Oncology. University of Duesseldorf

University of Duesseldorf Medical School. Duesseldorf. Germany

In order to optimize a retroviral transduction protocol for the genetic modification of NOD/SCID repopulating human umbilical cord blood (UCB) cells we initially compared four different cytokine cocktails for their ability to enable efficient transduction. CD34+ enriched UCB cells $(n=4)$ were prestimulated with different cytokine cocktails followed by a quadruple transduction procedure within 48 hours in fibronectin coated dishes, all in presence of the same cytokine cocktail used for prestimulation. A gibbon ape leukemia virus (GALV) pseudotyped murine stem cell virus (MSCV) based retroviral vector encoding the enhanced green fluorescence protein (EGFP) marker was used for transduction. FACS analysis of CD45+/EGFP+ cells revealed transduction rates of $72.7 \pm 2.9 \%$ for cytokine combination I (SCF, IL3, IL6), $49.6 \pm 21.8 \%$ for combination II (SCF, TPO, Flt-3), $60.7 \pm 16.6 \%$ for combination III (SCF, TPO, G-CSF), and $64.9 \pm 12.8 \%$ for combination IV (SCF, TPO G-CSF, Flt-3). Mean transduction rates of CD34+ ranged in between 29.3 and $38.0 \%$, revealing no statistical significance between the combinations tested. In further experiments duration of incubation period as well as number of transduction rounds were evaluated for their influence on engraftment of EGFP+ UCB cells in NOD/SCID mice. Best results were obtained with a 24 hour prestimulation period and a triple transduction procedure within 72 hours, followed by 24 hours of maintenance.

Therefore, in subsequent experiments $(\mathrm{n}=8)$ cytokine combination IV as well as the modified transduction protocol was applied in combination with the high titer GALV pseudotyped EGFP containing MESV/SFFV hybrid vector SFbeta11-EGFP. These experiments resulted in a mean transduction rate for CD45+ of $40.9+17.8 \%$ and $40.4+3.7 \%$ for CD34+ cells. Transplantation of $0.9-3.8 \times 10^{6}$ transduced cells into NOD/SCID mice resulted in a percentage of $17.2 \pm 14.6 \%$ human CD $45+$ cells in engrafted animals when analyzed by FACS four weeks after transplantation. $33.5 \pm 10.5 \%$ of engrafted human CD45+ cells were EGFP positive. Subpopulation analysis of the engrafted EGFP+ cells revealed $22.8 \pm 8.0 \% \mathrm{CD} 19+/ \mathrm{CD} 45+$ cells and $22.6 \pm 4.4 \%$ CD34+/CD45+ cells, the predominant subtype being CD33+/CD45+ with $45.7 \pm 12.3 \%$. In conclusion, our gene transfer protocol results in sufficient transduction of both, lymphoid and myeloid differentiated cells.

\section{Poster Session: Coagulation}

P490

\section{Thrombophilia and Spontaneous Abortion}

\section{'Zimmermann A. ${ }^{1}$, Kühnel G. ${ }^{1}$, Bohlmann M.K. ${ }^{2}$,} Kemkes-Matthes $B$.

'Zentrum Innere Medizin, Justus-Liebig-Universität Gießen, Germany ${ }^{2}$ Klinik für Frauenheilkunde und Geburtshilfe, Justus-Liebig Universität Gießen, Germany

Introduction: Antiphospholipid (APA)- syndrome is well known to be a risk factor for abortion. Moreover, other thrombophilic risk factors like Factor V Leiden mutation are discussed to enhance the risk of spontaneous abortion, too. Nevertheless, data concerning thrombophilia and spontaneous abortion still are poor. Thus, the aim of our study was to search for association between thrombophilic risk factors and spontaneous abortion. Patients and methods: 136 women who had experienced abortion(s) were examined for thrombophilic risk factors. Median age at time point of first abortion was 29,9 years $\pm 5,8$ (range: $16-42$ years). In all patients, in addition to routine laboratory tests, the following parameters were determined: PT, aPTT, fibrinogen, antithrombin (AT), aPC ratio, protein $\mathrm{C}$, protein $\mathrm{S}$, protein $\mathrm{Z}$, plasminogen, factor XII, factor VIII:c, lipoprotein (a), antiphospholipid-antibodies, cardiolipin-antbodies (ELISA IgM and IgG) and $\beta 2$-glycoprotein-antibodies. Moreover, patients were tested for Factor V Leiden (FVL)-mutation, MTHFR- (677T) and prothrombin-polymorphisms (G20210A). Results: 1 136 women experienced 314 miscarriages in total. 47/136 patients hadnon- recurrent, 89/136 had recurrent abortions.75\% of abortions occurred during the first trimester of pregnancy. 2. In 96/136 (70,6\%) patients, thrombophilic risk factors were determined: In 49/136 patients MTHFR-Polymorphisms (10 homozygous, 39 heterozygous) were detected, 16/49 MTHFR-Polymorphism patients additionally presented with FVL-Mutation. In 35/136 patients APCresistance (FVL-mutation) were detected (1 homozygous, 34 heterozygous), 16/35 FVL-patients additionally presented with MTHFR-Mutation. 18/136 patients had APA-syndrome, 2/136 patients presented with ProthrombinPolymorphisms (2 heterozygous), 2/136 patients had Protein-S-defiency, 2/136 patients presented with Antithrombin (AT3)-defiency, 2/136 patients presented with Thrombocytopenia, 1/136 patients presented with Factor XII defiency, 1/136 patients presented with Plasminogen defiency. Conclusion: In women who had experienced (recurrent) abortion(s), thrombophilic risk factors were detected very frequently - even more frequently than usually detected in venous thromboembolism. Our data show, that especially Factor $\mathrm{V}$ Leiden mutation and MTHFR polymorphism seem to play an importan role in this context. Incidence of APA syndrome (13\%) was comparable to data from earlier studies.

\section{P491 \\ Economic Evaluation of Anti-D Immunoglobulin for the Treatment of Immune Thrombocytopenic Purpura in German Adults}

Sclar D.A. ${ }^{1}$, Matzdorff A. , Skaer T.L. ${ }^{1}$, Robison L.M. ${ }^{1}$, Evans M.A. ${ }^{1}$, Li-McLeod J.M., Poulios N.S.

${ }^{1}$ College of Pharmacy, Washington State Univ., Pullman, USA,

${ }^{2}$ Dpt. of Hematology/Oncology, Caritasklinik Saarbruecken, Germany

Purpose: Immune thrombocytopenic purpura (ITP) is an autoimmune disorder characterized by a low platelet count and mucocutaneous bleeding. The estimated incidence is 100 cases per 1,000,000 persons per year. Anti-D immunoglobulin (anti-D) is an alternative to intravenous immunoglobulin (IVIG) for the treatment of ITP in Rh+ patients. The objective of this economic evaluation was to compare the total mean cost of care for treating $\mathrm{Rh}+$ ITP patients, by way of a treatment algorithm that initiates therapy with steroid, and moves to either anti-D, or IVIG. Methods: This economic analysis was conducted from a payer's perspective. Modeling (Monte Carlo Simulation) was undertaken to examine the mean total cost of care treating $\mathrm{Rh}+$ ITP patients between the ages of 15-80 years ( 5 year increments). The model structure followed the ITP treatment pathway as advanced by Cines and Blanchette (NEJM, 2002), and was adapted to German practice patterns via in-depth interviews with local medical experts. Health resources consumed included pharmacotherapy (steroids; IVIG; anti-D), splenectomy, hospitalization, and physician care. Results: When comparing two simulated cohorts (100,000 iterations), anti-D therapy after steroid failure was associated with 48,647 fewer hospitalization days (reduced administration time) and 373 fewer splenectomies, as compared to IVIG therapy after steroid failure. Use of anti-D was associated with a $25 \%$ decrease in overall treatmen costs, across all age groups. Conclusions: Anti-D immune globulin offers an efficacious and fiscally prudent alternative to IVIG for the treatment of ITP among $\mathrm{Rh}+$ patients

P492

\section{Proteinase-Activated Receptor-1 Expression in Primary Human Carcinoma Cells}

\section{Xie W.-Z. ${ }^{1}$, Leibl M. ${ }^{1}$, Clark M. ${ }^{1}$, Kunze $T^{2}{ }^{2}$, Steiner $M^{3}$} Dohrmann P. , Gieseler F. ${ }^{1 *}$

${ }^{1}$ Department of Internal Medicine, University of Kiel, Germany, ${ }^{2}$ Department of Pharmacy, University of Kiel, Germany, ${ }^{3}$ Institute of Clinical Chemistry and Pathobiochemistry, University of Rostock, Germany, ${ }^{4}$ Department of Thoracic and General Surgery, University of Kiel, Germany

Cancer patients, especially with lung cancer and gastrointestinal tumors, have an increased incidence of thromboembolic complications. Clinical evidence has shown that activation of thrombin occurs regularly in the natural course of cancer. Thrombin-mediated activation of cellular receptor(s), such as proteinase-activated receptor-1(PAR-1), has important functions in tumor growth, local progression, and distant metastasis.

During tumor progression, many patients develop malignant effusions. Treatment is complicated by increased resistance of tumor cells to chemotherapy. 
Our hypothesis is that activated thrombin within malignant effusions results in creased proliferation of tumor cells mediated through cleavage of PAR-1. We suggest that these cells might have a survival benefit.

We harvested primary cancer cells from the pleural and peritoneal effusions of patients with pancreatic carcinoma, colon carcinoma, lung cancer (nonsmall cell lung cancer as well as small cell lung cancer), carcinoid and pleura mesothelioma. We found that $55.6 \%$ of primary cancer cells were PAR-1 positive using RT-PCR assays. Correspondingly, these cells were positive using the FCM (flow cytometry) assay.

In this paper, we show for the first time that half of the primary cancer cells exhibit a positive PAR-1 expression. Also, thrombin is activated in most malignant effusions as shown by prothrombin fragment 1,2 elevation. These cell-biological mechanisms might aggravate cellular resistance in primary tumor cells found in malignant effusion in end-stage cancer.

\section{Poster Session: Stem Cell Transplantation I}

P493

\section{Functional and Phenotypic Characterization of NK Cells in AML Patients after Allogeneic Stem Cell Transplantation (SCT)}

\section{Multhoff G., Gross C., Holler E., Dickinson A., Andreesen R.} University Hospital Regensburg, Germany

Purpose: Hsp70 is frequently found on the plasma membrane of bone marrow-derived leukemic blasts but not on normal bone marrow cells. In vitro Hsp70-peptide activated NK cells have been found to lyse autologous Hsp70 membrane-positive leukemic blasts (Gehrmann et al 2003). Granzyme $B$ release provides a surrogate marker for estimating the cytolytic response of NK cells against Hsp70 membrane-positive tumor target cells (Gross et al 2003). Here, we studied the phenotype and cytolytic capacity of NK cells derived from AML patients at different time points (days 14-20, 45, 90, 6 months, 12 months) after allogeneic stem cell transplantation (SCT). Methods: HLA class I , HLA-E and Hsp70 surface expression was determined on patient-derived leukemic blasts by flow cytometry. The amount of $\mathrm{NK}$ and $\mathrm{T}$ cells and their phenotype was investigated by multicolor flow cytometry using the following antibody-combinations detecting classical NK cell specific markers and activatory killer cell receptors: CD3/CD16/CD56, CD56/CD94, CD161/CD69, NKp30, NKp44 and NKp46. Concomitantly, the cytolytic activity was analyzed in unstimulated and Hsp70-peptide activated, patient-derived PBL, against leukemic blasts and K562 cells by granzyme B ELISPOT technique. Results: Leukemic blasts were positive for HLA class I HLA-E and Hsp70. A significant amount of CD3-negative, CD56/CD94positive NK cells, but hardly any CD3-positive $\mathrm{T}$ cells, were detectable between days 14 and 45 after allogeneic SCT. Although only few patients were tested so far, in all cases the lytic capacity, after in vitro stimulation with Hsp70-peptide, correlated with the expression of CD56/CD94 on allogeneic NK cells. Conclusions: In addition to autologous NK cells, also patientderived, allogeneic Hsp70-peptide activated NK cells, obtained between days 14 to 45 days post transplant, efficiently kill Hsp70 membrane-positive leukemic blasts. Further kinetic analysis should elucidate the graft-versusleukemia effect of NK cells at later time points after allogeneic stem cell transplantation. Project funded by EU-TRANS-EUROPE grant QLK3-CT2002-01936.

References: Gehrmann M, Schmetzer H, Eissner G, Haferlach T, Hiddemann W, Multhoff G. Membrane-bound Hsp70 in AML: a tumor specific recognition structure for the cytolytic activity of autologous NK cells. Haematologica 88:473-475, 2003

Gross C, Koelch W, Arispe N, DeMaio A, Multhoff G: Cell surface-bound Hsp70 mediates perforin-independent apoptosis by specific binding and uptake of granzyme B. JBC 17: 41173-41181, 2003
P494

Veno-Occlusive Disease of the Liver is Uncommon after Conditioning with Low-Dose Total Body Irradiation

Grommisch L., Hegenbart U., Al-Ali H.-K., Lange T., Pönisch W., Raida M., Leiblein S., Gentilini C., Schönfelder U., Heyn S., Schwittay M., Niederwieser D.

${ }^{1}$ Division of Hematology/Oncology, Department of Internal Medicine II, University of Leipzig, Germany

Purpose: Hepatic veno-occlusive disease (VOD) of the liver is a life-threatening complication after allogeneic haemopoietic cell transplantation (HCT). It occurs in 10 to $60 \%$ of patients undergoing high-dose chemotherapy and HCT. Severity ranges from a mild reversible to a severe disease cumulating in multiorgan failure (MOF) and death. Reduced-intensity conditioning (RIC) regimens for allogeneic HCT were introduced to reduce the morbidity and mortality of conventional HCT. VOD was until now uncommonly reported after RIC. Methods: In a series of 215 patients transplanted after conditioning with low-dose total body irradiation (TBI) and fludarabine (FLU) we observed 2 patients (pt.) who developed VOD. Patient 1, 51 years, and pt. 2 , 59 years, received RIC because of Ph-neg CML, advanced phase, and highrisk-MDS-relapse after first conventional HCT respectively. The donors were unrelated in pt. 1 and related in pt. 2. Conditioning regimen consisted of fludarabine $30 \mathrm{mg} / \mathrm{m}^{2}$ from day -4 to -3 and 2 Gy TBI (pt. 1) or 3 Gy TBI (pt. 2) on day 0. Immunosuppressive therapy included cyclosporine and mycophenolate-mofetil. Results: Pt. 1 and pt. 2 developed the classical symptoms of VOD [painful hepatomegaly, jaundice (bilirubin $838.3 \mu \mathrm{mol} / \mathrm{l}$ in pt. 1 and $119.9 \mu \mathrm{mol} / 1$ in pt. 2 , normal range $2.0-20.5 \mu \mathrm{mol} / \mathrm{l})$, and weight gain] 6 and 40 days after HCT respectively. Pt. 1 died because of MOF on day 13. Pt. 2 was successfully treated with defibrotide. He recovered from moderate VOD after 34 days of treatment. Conclusions: We conclude that VOD is an uncommon complication after RIC-HCT. In our series of 215 patients, the incidence was $<1 \%$. This is much lower than that reported after conventional HCT. Although rare, VOD must be considered in patients with typical symptoms after non-myeloablative HCT.

\section{P495 \\ Quantitative Assessment of Hematopoietic Chimerism afterAllogeneic Stem Cell Transplantation via SNP Real- Time PCR}

Zach O., Krieger O., Kessler B., Kasparu H., Machherndl-Spandl S., Girschikofsky M., König J., Hauser H., Lutz D.

Elisabethinen Hospital, 1st Dept. of Inernal Medicine, Linz, Austria

Purpose: After allogeneic stem cell transplantation (SCT), distinguishing between host- and donor-hematopoiesis (chimerism) is critical to observe the engraftment of donor stem cells and to ensure the appropriate use of donor lymphocyte infusions. Unitl now various techniques have been used to document chimerism, such as FISH with XY chromosome-specific probes or PCR-based amplification of VNTRs. However, XY-FISH is restricted to sexmismatched allografts and VNTR analysis has low sensitivity as a consequence of PCR competition biases. Single nucleotide polymorphisms (SNPs) represent a source of human genetic diversity, therefore it is possible to distinguish individuals from one another through SNP analysis. Methods: PCR conditions of selected SNP-loci allow the amplification of one specific DNA-sequence, all other possible SNP-sequences lead to negative results. In the first step, specific SNP-loci for host and donor DNAs are selected and used as markers for quantification via TaqMan probes and LightCycler technology. Results: Until now 27 SCT recipients and their HLA-identical donors were screened for specific SNP-markers using a panel of 30 SNP-loci. For all pairs at least one SNP-locus specific for donor and host DNA, respectively, was identified and used in the follow up of the patients after Tx. Sixteen patients received standard condition (8 AML, $2 \mathrm{Ph}+\mathrm{ALL}, 2 \mathrm{Ph}+$ CML, 1 ALL, 1 CLL, 1 MDS, 1 SAA) and 11 patients reduced intensity (4 solid tumors, 3 AML, 2 NHL, 1 MM, 1 Ph+ ALL). Peripheral blood samples from 7 patients were examined by VNTR- and SNP-analysis in parallel. The detection limit of host-cells was $5 \%$ for VNTR PCR and $0,005 \%$ for quantitative SNP real-time PCR. In addition dynamic changes in the proportion of recipient cells were observed in patients with VNTR donor genotypes, indicating the possibility of early detection of relapses via SNP-quantification through an increased sensitivity. From $2 \mathrm{Ph}+\mathrm{ALL}$ patients results from bcr/abl quantification and SNP real-time PCR after Tx were available. Both markers had a parallel course during follow up, whereby bcr/abl had a sensi- 
tivity of $0,0003 \%$ and is a specific marker for tumor cells whereas SNPs are specific for host-cells independent of their origin. Conclusions: The SNP real-time PCR is a fast and reliable method for quantitative assessment of hematopoietic chimerism after allogeneic SCT. The sensitivity is 3 logs higher compared to VNTR PCR and offers the possibility of early detection of relapses.

\section{P496}

\section{Effects of Prior Irradiation and Chemotherapy on the Mobilisation of PBPCs in Patients with Multiple Myeloma}

Rinn J. ${ }^{1}$, Schwella N. ${ }^{1}$, Wollmer E. ${ }^{1}$, Neubauer A. ${ }^{1}$, Heinzel-Gutenbrunner $M^{2}{ }^{2}$, Straßmann G. $^{3}$, Ritter $M$.

${ }^{1}$ Klinik für Innere Medizin mit Schwerpunkt Hämatologie, Onkologie und Immunologie, Philipps-Universität Marburg, Germany, ${ }^{2}$ Institut für Biometrie und Medizinische Epidemiologie, Philipps-Universität, Marburg, Germany, ${ }^{3}$ Klinik für Strahlentherapie und Radioonkologie, Philipps-Universität Marburg, Germany

Purpose: Irradition of bone marrow prior to mobilisation of autologous peripheral blood progenitor cells (PBPC) may lead to reduced yield of mobilized CD34+ cells in patients with multiple myeloma. Furthermore, clinical parameters like previous chemotherapy regimens and patient's characteristics were taken into account. Patients and Methods: 122 patients (76 male, 46 female) were analysed retrospectively. The median age was 52 years (range 33-68) at the time of PBPC mobilisation. 60\% disease at stage 3 (Salmon and Durie) by the time of diagnosis. 53 patients had been irradiated prior to mobilisation chemotherapy. Most patients $(87 \%)$ were mobilised with highdose cyclophosphamide followed by G-CSF. Conventional-dose chemotherapy prior to mobilisation consisted of vincristin, adriamycin and dexamethason (VAD) in 51 patients, VAD + cyclophosphamide (VACD) in 38 patients, melphalan (17 patients) or other chemotherapy regimens ( 8 patients). According to hematological toxicity, the previous cytotoxic treatment was divided into 3 groups: moderately myelotoxic $(<5$ cycles, no melphalan) intermediate ( $>5$ cycles, no melphalan) and highly myelotoxic chemotherapy (prior treatment with melphalan). Dose and fractionation of irradiation, volume of the irradiated bone marrow, effective biological dose and time between radiation therapy and mobilisation were taken into account Results: 114 patients were analysed, 8 had to be excluded due to partially missing data files. The median volume of irradiated bone marrow was $8,5 \%$ (range 1-30\%) of the hematopoetic bone marrow and the median dose was 36 Gy (19-60 Gy). Correlation of irradiated bone marrow volume and number of CD34+ cells in peripheral blood $(\mathrm{p}=0.78)$, dose of bone marrow irradiation and CD34+ cells $(\mathrm{p}=0.4)$ showed no significant result. Multivariate analysis showed significant differences when comparing the different chemotherapygroups $(\mathrm{p}=0,001)$ and the patient's age $(\mathrm{p}=0,031)$. Conclusions: In this study local irradiation of bone marrow prior to PBPC mobilisation did not lead to a reduced level of circulating CD34+ cells in the peripheral blood on the firstday of leukapheresis. However, there seems to be a trend for low CD34+ cell counts in patients with a higher proportion of irradiated bone marrow volume. The dose of irradiation had no impact on CD34+ cell count. Thus, we could not show any significant negative impact induced by prior radiotherapy on the mobilisation of PBPCs in patients with multiple myeloma.

\section{P497}

\section{CNS Involvement Of Tropheryma Whipple: The First Description of a Late Complication after Allogeneic Bone Marrow Transplantation}

\section{Yaman A., Burlakova I., Schwerdtfeger R., Schleuning M.} KMT-Zentrum, Deutsche Klinik für Diagnostik Wiesbaden, Germany

Background: Whipple's disease (WD) is an extremely rare systemic disease of infectious etiology. About 120 cases of WD with nervous system involvement are reported in literature. We describe the first case of a cerebral manifestation after allogeneic bone marrow transplantation (BMT). Case Report: A 36 year old man undergoing matched related allogeneic BMT for CML developed 42 months after transplantation signs of motorical and sensorica deficit on the right hand side (Nervus ulnaris). MRI showed three intracerebral mass lesions in the left hemisphere with typical signs suggestive of toxoplasmosis. Despite immediately starting toxoplasmosis therapy with Daraprime, Sulfadiazine and Clindamycine the neurologic symptoms worsend and lesions increased. Therefore a stereotactic biopsy was performed. Histology showed PAS-positiv macrophages as typical signs of a Tropheryma whippeli infection. The diagnosis was confirmed by electron microscopy. The PCR diagnosis is pending. No signs of other infectious diseases or malignancy were observed. Immediately after starting a combination therapy of ceftazidime and streptomycine intracerebral lesions regressed and the patient resolved of all neurological symptoms. Summary: Neurologic presentation of WD is very rare. This is the first case of a cerebral WD after BMT. To avoid a delay of therapy the stereotactic biopsy is indispensable. Antibiotic treatment for at least 1 year is recommended.

\section{P498}

NKT cells are Significantly Increased up to one Year after Allogeneic as Compared to Autologous Stem Cell Transplanta tion and thus May Contribute to Graft-versusLeukemia Activity

Uharek L., Bonitz A., Hilbers U., Gentilini C., Niederwieser D. Charité Berlin-Campus Benjamin Franklin, University Hospital Leipzig, Germany

Purpose: NKT cells are distinct cell populations sharing characteristics of both T cells and natural killer (NK) cells. Since experimental and clinical data indicate that they are involved in tumor surveillance, we compared the number of different types of NKT cells $(\gamma \delta$ NKT, CD3+CD56+ NKT, and $\mathrm{V} \alpha 24 \mathrm{~V} \beta 11 \mathrm{NKT})$ at defined time points after autologous and allogeneic HSCT. Methods: In a prospective study, the absolute and relative number of NKT subsets from pts with AML, ALL ( $\mathrm{n}=11)$, NHL, HD, MM ( $=15)$, and CML $(n=5)$ were determined by three colour flow cytometry and compared with classical T- and NK-cell subsets immediately prior to and at day +30 , day +100 , and day +300 following autologous $(n=15)$ or allogeneic $(n=16)$ HSCT. Results: The median number of $\gamma \delta \mathrm{NKT}, \mathrm{CD} 3+\mathrm{CD} 56+\mathrm{NKT}$, and V $\alpha 24$ V $\beta 11$ NKT was significantly higher in stem cell donors than in pts prior to HSCT. After autologous transplantation, CD4+ and CD3+ cells improved rapidly, whereas all types of NKT cells remained at extremely low levels until day +300 . In contrast, recipients of allografts demonstrated a steady increase in the number of all NKT cell subtypes. At day 30, $\gamma \delta$ NKT, CD3+CD56+ NKT, and V $\alpha 24 \mathrm{~V} \beta 11$ NKT were significantly higher in the allografted vs autografted patients $(2,8$ vs. $17,8 / \mathrm{nl}, \mathrm{p}<0.05 ; 3,5$ vs. $17,2 / \mathrm{nl}, \mathrm{p}<0.05 ; 50$ vs $110 / \mathrm{pl}, \mathrm{p}<0.05)$. The difference was even more pronounced at day +300 . No significant differences were detected in the number of CD56 cells and in the percentage of NK cells expressing the activation markers NKp30, NKp44 and NKp46. Conclusion: NKT cell subsets are decreased in pts with different types of hematological malignancies. Whereas their number remains extremely low after autologous transplantation, allografting resulted in increasing numbers of all NKT subsets. Whereas this difference may contribute to GvL activity and wheather the number of NKT cells is related to GvHD or other clinical parameters will be determined in a more homogenous population of AML patients after allogeneic HSCT.

P499

Cyclosporine A and Mycophenolate Mofetil Versus Cyclosporine A and Methotrexate for Graft Versus Host Prophylaxis after Stem Cell Transplantation from HLA-Identical Siblings

Neumann F., Gräf T., Tapprich C., Vaupel M., Steidl U., Pape H., Germing U., Haas R., Kobbe G.

Dept. of Hematology, Oncology and Clinical Immunology, Heinrich Heine University Duesseldorf, Germany

Purpose: The combination of Cyclosporin A (CSA) and Methotrexate (MTX) is an effective and well established combination for the prevention of graft versus host disease (GVHD) after allogeneic stem cell transplantation (SCT). Mycophenolate Mofetil (MMF) is an inhibitor of the purine nucleotide synthesis and inhibits proliferation of activated lymphocytes. We retrospectively compared the combination of CSA and MTX versus CSA and MMF in patients with acute and chronic leukaemia after allogeneic SCT from HLA identical siblings (HIS). Methods: One hundred eleven patients (median age 34 , male 58, female 53) with acute myeloid $(\mathrm{n}=48)$ and acute lymphoblastic $(n=21)$ as well as chronic myeloid leukaemia $(n=42)$ received either CSA/MMF $(n=30)$ or CSA/MTX $(n=81)$ as GVHD prophylaxis following high dose chemotherapy alone $(n=40)$ or in combination wit TBI $(n=71)$ and consecutive allogeneic SCT from HIS. Eighty-eight patients were 
in complete remission (CR) or chronic phase (CP) while 23 patients had a progressive disease. Median follow up time after transplantation was 12 months (range 1-67 months) in the CSA/MMF group and 42 months (range 0,5-166) in the CSA/MTX group. Results: Although results showed a trend in favour of the CSA/MMF combination, no statistically significant difference was found in the overall survival of the two treatment groups with 2 year survival rates between $65 \%$ (CSA/MTX) and 70\% (CSA/MMF) in patient with $\mathrm{CR}$ or $\mathrm{CP}$, and $20 \%$ in the group of patients with advanced disease respectively. As well no differences in relapse rate, treatment related mortality or death from disease were evident. Moreover, the incidence of acute GVHD and chronic GVHD was the same among the two regimens. Interestingly, the time until leukocyte reconstitution differed significantly within the two treatment groups with 11 days (median, range 3-17) in patients receiving CSA/MMF in $\mathrm{CR}$ or $\mathrm{CP}$ and 18 days (median, range 12-62) in the CSA/MTX group. This difference was also found in patients with advanced disease (CSA/MMF median 14 days, range 1-102, CSA/MTX median 18,5 days, range 17-27). Conclusion: results from this retrospective single centre analyses suggest that the combination CSA/MMF is at least equivalent to CSA/MTX for prophylaxis of GVHD for patients receiving SCT from HIS with no change in overall survival, relapse rate or treatment related mortality. The time to leukocyte recovery is reduced by the use of MMF in stead of MTX in combination with CSA

\section{P500}

\section{Second Line Treatment of Acute GrHD with Cytokine Blocking Agents May Improve Short Term Outcome - A Retrospective Analysis in 169 Consecutive Patients}

\section{Holler E. ${ }^{1}$, Hahn J. ${ }^{1}$, Pihusch M. ${ }^{1}$, Finger F. ${ }^{1}$, Wolff D. ${ }^{2}$,} Andreesen $R^{1}{ }^{1}$

${ }^{1}$ University Hospital Regensburg, Germany, 2University Hospital Rostock, Germany

Purpose: Whereas corticosteroids can be considered as standard for first line treatment of acute GvHD following allogeneic stem cell transplantation, there is no clearly defined and recommended second line treatment. Therefore, we now analysed treatment related mortality and outcome in relation to first and second line treatment of GvHD. Methods: Records of a consecutive cohort of 169 patients receiving an allogeneic stem cell transplant at the university of Regensburg between 6/1998 and 10/2003 were analysed. Due to a high rate of mainly infectious toxicity of second line treatment using $\mathrm{T}$ cell depleting agents like ATG or complete TNF blocking agents like infliximab (group I) we switched our strategy to the combined use of cytokine blocking agent daclizumab and etanercept (group II) in 2002. Results: First line treatmen with corticosteroids was given in 125 of 166 evaluable patients $(75 \%)$ whereas a total of $44(27 \%)$ patients required second line treatment due to progression of GvHD. TRM at 6 months was $19 \%$ in pts not receiving corticosteroids and $18 \%$ in those receiving corticosteroids alone but increased to $57 \%$ in patients receving $2^{\text {nd }}$ line treatment. Within this group, $180 \mathrm{~d}$ TRM was lower in group I patients treated with etanercept and daclizumab $(32 \%)$ or daclizumab alone $(40 \%)$ than in patients receiving depleting antibodies (57\%) (p 0.07) However, due to an increased incidence of late complications and relapse overall actuarial survival after a median follow up of 19 months was $27 \%$ in group I and $22 \%$ in group II. Conclusions: Our data suggest that cytokine blocking agents might be less toxic but clearly support the need of randomised and standardized comparisons of $2^{\text {nd }}$ line strategies. Therefore, a multicenter trial comparing both approaches in steroid-resistant GvHD has been initiated.

\section{P501}

\section{Acute Graft-versus-host Disease Prophylaxis Using a Combination of Alemtuzumab and Ciclosporin: Comparison Between Two Dose Levels of Alemtzumab}

Biersack H., Peceny R., Elmaagacli A., Trenschel R., Beelen D.W. Department of Bone Marrow Transplantation, University Hospital Essen, Germany

Alemtuzumab (Campath-1H), a humanized monoclonal antibody directed against the CD52 antigen, has demonstrated particular high efficacy in the prevention of acute graft-versus-host disease (GvHD). It is generally thought that its major mechanism is in-vivo donor T-cell depletion (TCD). In addition, Alemtuzumab can act on host antigen-presenting cells (APCs) and may thereby inhibit the induction phase of acute GvHD. At the commonly applied total dose level of $100 \mathrm{mg}$ Alemtuzumab, both mechanisms appear to be involved, while after low-dose Alemtuzumab (i.e. $50 \mathrm{mg}$ total dose) its effect on host APCs appears to prevail. To evaluate the clinical efficacy of the two dose levels, two cohorts of patients (pts) received either standard dose $(\mathrm{n}=36)$ or low dose $(n=34)$ Alemtuzumab in combination with ciclosporin (3 $\mathrm{mg} / \mathrm{kg}$ /day IV). Both cohorts were comparable in terms of underlying diseases as well as patient, donor and treatment characteristics (advanced stages of hematologic malignancies $n=60$, median age $46 \mathrm{yrs}$, median donor age 38 yrs, female to male transplants $n=13$, matched unrelated donors $n=60$, sibling donors $\mathrm{n}=10$, myeloablative conditioning regimen $\mathrm{n}=70$ ). Alemtuzumab was applied as a two-hour infusion on days -6 to -2 . No serious acute adverse effects were noted during a total of 350 antibody infusions. The clinical grades of severity of acute GvHD were nearly identical between the two Alemtuzumab dose levels (50 mg/100 mg): grades 0: 85\%/80\%, I: $9 \% / 14 \%$ II: 3\%/3\%, III-IV: 3\%/3\%. Consequently, transplant-related mortality (TRM) at day 100 was comparatively low with $8 \% \pm 5 \%(32 / 34$ survivors) after 50 $\mathrm{mg}$ and $11 \% \pm 5 \%$ (32/36 survivors) after $100 \mathrm{mg}$. The 1 -yrs survival estimate for pts in early disease stages is $83 \% \pm 15 \%$ (9/10 survivors [90\%]). This estimate is $50 \% \pm 13 \%$ (44/60 survivors [73\%]) in advanced disease stages. Causes of TRM were bacterial/mykotic infections $(n=6[9 \%])$, multiorgan failure $(n=3[4 \%]), E B V-L P D$, acute cardic arrest, and leukencephalopathy (each $n=1[1.4 \%]$ ), while 5 pts have died from disease recurrence $(n=5[7 \%])$. In conclusion, both investigated total dose levels of Alemtuzumab are comparatively effective in preventing acute GvHD and are associated with a reasonable short-term outcome in a predominantly unfavorable patient population. Further, our experience indirectly supports that the inactivation of host APCs by Alemtzumab is the dominant mechanism in preventing acute GvHD.

\section{P502}

\section{T-cell Chimerism after Transplantation of Allogeneic Highly Purified Peripheral Blood CD34+ Cells from HLA- identical Sibling Donors in Chronic Myeloproliferative Syndromes Compared to Acute Leukemias}

\section{Peceny R., Elmaagacli A.H., Trenschel R., Ottinger H., Beelen D.W. \\ University Hospital Essen, Germany}

Purpose: In the setting of transplantation using highly purified peripheral blood CD34+ cells from HLA-identical sibling donors without any other prophylactic immunosuppression, we studied the development of chimerism using fluorescence in situ hybridization for the $\mathrm{X}$ and $\mathrm{Y}$ chromosomes. Methods: Seventy one adult patients were transplanted for malignant hematologic diseases, 56 suffering from chronic myeloproliferative syndromes (MPS) mainly CML, 8 acute leukemias (AL), and 7 lymphomas or multiple myelomas. Severe acute graft-versus-host disease did not occur post transplant. Fifty eight patients at least 3 months after the first donor lymphocyte infusion (DLI) received a median of 3 DLI starting on day $93(44-986)$ in a median maximal dose of $3.3 \times 10^{\wedge} 6 \mathrm{CD} 3+$ per $\mathrm{kg}$ body weight. In the last 36 CML patients, DLI were started as a programmed T-cell-addback (TCA) on days +90 and +135 in doses of $0.33 \times 10^{\wedge} 6$ and $1 \times 10^{\wedge} 6 \mathrm{CD} 3+$ per $\mathrm{kg}$ Results: NK-cells recovered within the first month. Numbers of B-cells and CD8+ cytotoxic T-cells normalized in the 2nd month post transplant whereas T-helper cell numbers stayed low beyond the first year (Beelen et al., 2000). Twenty nine patients had a donor patient gender disparity and a follow up of at least 3 months, 16 of these received donor lymphocytes as TCA. In contrast to a rapid complete chimerism of the NK- and B-cells in all patients, overall and T-cell chimerism developed differently in patients suffering from MPS compared to AL. Overall chimerism stayed in the MPS group at $90 \%$ in the first year after transplant, whereas overall chimerism in the AL group was > $99 \%$ at all time points analyzed $(\mathrm{p}<0.05$ at 3,6 and 9 months, $\mathrm{p}=0.065$ at 12 months). T-cell chimerism in the MPS group was low with $5 \%$ at 3 months, increasing to $40 \%$ at 6 months, $48 \%$ at 9 months, $62 \%$ at 12 months, reaching $87 \%$ at 18 months. AL patients were completely chimeric in the T-cell population shortly after transplant ( $\mathrm{p}=0.006$ at 3 months). Early T-cell addback did not make a difference. The T-cell chimerism in the groups with TCA vs. late DLI was $38 \%$ vs. $41 \%(\mathrm{p}=0.52)$ at 6 months, $40 \%$ vs. $71 \%(\mathrm{p}=0.60)$ at 9 months and $45 \%$ vs. $87 \%(\mathrm{p}=0.51)$ at 12 months. Conclusions: Expansion of patient clones is responsible for the early recovery of CD8+ T-cells in patients suffering from chronic myeloproliferative syndromes in contrast to acute leukemia patients. This might explain the known increased risk of secondary graft failure in this patient group. 
P503

\section{No Predictive Value of Cytotoxic or Helper Precursor T Cell Frequencies for Outcome when Analyzed from the Graft after Stem Cell Transplantation}

Kircher B. ${ }^{1}$, Niederwieser $D .{ }^{2}$, Gächter A. ${ }^{1}$, Lätzer K. ${ }^{1}$, Eibl G. ${ }^{3}$, Gastl G. ${ }^{1}$, Nachbaur D. ${ }^{1}$

${ }^{1}$ Laboratory For Tumor And Immunobiology And Bone Marrow Transplantation Unit, Division Of Hematology \& Oncology, Innsbruck Medical University, Innsbruck, Austria, ${ }^{2}$ Div. Hematology \& Oncology Dept. Of Internal Medicine II, Leipzig University, Leipzig, Germany, ${ }^{3}$ Dept. Of Biostatistics And Documentation, Innsbruck Medical University, Innsbruck, Austria

The predictive value of limiting dilution analyses (LDA) measuring cytotoxic and helper T lymphocyte precursor (CTLp and HTLp) frequencies for outcome after stem cell transplantation (SCT) is still a matter of debate. One reason may be that CTLp and HTLp frequencies are determined in periphera blood mononuclear cells (PBMC) and this responder cell population does not reflect the cell type composition of the graft.

We assessed whether CTLp and HTLp LDA can predict complications after human leukocyte antigen (HLA)-identical SCT when CTLp and HTLp frequencies are analyzed in PBMC of the respective stem cell graft (bone marrow (BMMC) or granulocyte colony-stimulating factor (G-CSF)-mobilized PBMC) and compared to PBMC of PB. Host-specific CTLp frequencies measured in 25 patients and HTLp frequencies analyzed in six patients were low in all responder cell sources. CTLp and HTLp frequencies seen agains HLA-mismatched unrelated third-party cells were high, but third-partyspecific CTLp and HTLp frequencies were lower in G-CSF-PBMC than in PBMC ( $p=0.047$ for CTLp frequencies). Host-specific CTLp frequencies analyzed in all responder cell sources did not predict acute or chronic GvHD. Lower CTLp frequencies were detected in all responder cell sources from patients who relapsed after SCT than in patients without relapse, but the differences between both groups were statistically significant only in PBMC. In conclusion, a significant correlation was detected only between relapse and CTLp frequencies measured in PBMC. The lower frequency of third-partyspecific cells in G-CSF-PBMC indicates that the mobilization procedure with G-CSF itself may influence results.

\section{P504}

\section{Granzyme A and B Production Levels for the Selection of Stem Cell Donors}

Kircher B. ${ }^{1}$, Hack C.E. ${ }^{2}$, Dickinson A.M. ${ }^{3}$, Wang X.N. ${ }^{3}$, Oudshoorn M. ${ }^{4}$, Goulmy E. ${ }^{4}$

'Laboratory for Tumor and Immunobiology, Division of Hematology \& Oncology, Innsbruck Medical University, Innsbruck, Austria, ${ }^{2}$ Central Laboratory of the Netherlands Red Cross Blood Transfusion Service, Amsterdam,

The Netherlands, ${ }^{3}$ University Department of Hematology, School of Clinical and Laboratory Sciences, University of Newcastle upon Tyne, United Kingdom, ${ }^{4}$ Department of Immunohematology and Blood Transfusion, Leiden University Medical Center, Leiden, The Netherlands

Graft-versus-host disease (GvHD) due to host-reactive antigenic differences remains an important cause of morbidity and mortality after allogeneic stem cell transplantation (SCT). Currently, there is no reliable test system available to predict the development of this serious complication.

Activated donor cytotoxic $\mathrm{T}$ lymphocytes and natural killer cells produce granzymes (Gr) which are involved in the pathogenesis of GvHD. Therefore, we investigated a potential role of GrA and GrB production levels both for the selection of the best stem cell donor and the prediction of the development of acute GvHD prior to SCT.

We measured the GrA and GrB production levels in the supernatants of 96 hours pre-transplant mixed lymphocyte cultures (MLC) by enzyme linked immunosorbant assay (ELISA). GrA and GrB production levels from a selected cohort of 37 potential patient/donor pairs were correlated with relative responses (RR) of MLC and with HLA class II mismatches. In a second, consecutive cohort of 20 sibling SCT recipients an association of enhanced GrA and GrB production levels with the development of acute GvHD was investigated.

In vitro measurement of GrA and $\mathrm{GrB}$ production levels significantly correlated with the RR of pre-transplant MLC $(r=0.492, p \leq 0.01$ and $r=0.853$, $\mathrm{p} \leq 0.01$, respectively) and increased with the number of HLA class II mismatches between patient and donor. Pre-transplant GrA production levels were significantly associated with the in vivo development of acute GvHD grades II-IV in patients transplanted with an HLA-identical sibling donor $(\mathrm{p} \leq 0.001)$.

In conclusion, in vitro GrA and GrB production levels can be measured by a quantitative and sensitive ELISA. This novel and simple method may be used for functional selection of unrelated stem cell donors and for the identification of patients who are at risk for acute GvHD grades II-IV.

\section{P505}

\section{HA-1 as Target of the Graft-versus-Leukemia Effect}

Kircher B. ${ }^{1}$, Wolf M. ${ }^{2}$, Stevanovic S. ${ }^{3}$, Rammensee H.G. ${ }^{3}$, Grubeck-Loebenstein B. ${ }^{2}$, Gastl G. ${ }^{1}$, Nachbaur D. ${ }^{1}$

${ }^{1}$ Laboratory for Tumor- and Immunobiology and Bone Marrow Transplant Unit, Div. Hematology \& Oncology, Innsbruck Medical University, Innsbruck, Austria, ${ }^{2}$ Institute for Biomedical Aging Research, Austrian Academy of Sciences, Innsbruck, Austria, ${ }^{3}$ Institute for Cellular Biology, Dept. of Immunology, University of Tuebingen, Germany

Immunocompetent allo-reactive donor $\mathrm{T}$ lymphocytes mediate potent antileukemic activity, the graft-versus-leukemia (GvL) effect, during allogeneic stem cell transplantation (SCT) and/or donor lymphocyte infusion (DLI) in relapse after SCT. Target antigens of the GvL effect that are being discussed include leukemia-specific antigens such as bcr/abl, leukemia-associated antigens such as PR-1 and/or minor histocompatibility antigens (mHag). However, in the case of $\mathrm{mHag}$, their association with graft-versus-host disease (GvHD) makes it difficult to discriminate between $\mathrm{GvH}$ and $\mathrm{GvL}$ activity.

We have analyzed a GvL effect in a patient with Philadelphia chromosome positive acute lymphoblastic leukemia (ALL) who showed complete cytogenetic and molecular remission three months (during GvHD) and thirty months (without any signs of active GvHD) after DLI. Similar amounts of P190 bcr/abl- and HA-1-positive cells were detected in the patient at both time points by dimer staining and flow cytometry. HA-1-specific T cell clones were generated by incubating the patient remission cells during GvHD with dendritic cells of the patient before SCT and a CD8-positive, cytotoxic T cell line was established by sorting HA-1 dimer-staining remission cells (without GvHD) and further culture with patient pretransplant cells as stimulators. All HA-1-specific T cells lysed ALL, acute and chronic myeloid leukemia blasts and did not recognize HA-1-negative target cells. No P190 bcr/abl peptidespecific $\mathrm{T}$ cells could be expanded by repeated restimulations with patient pretransplant cells probably due to the weak immunogenicity of the P190 bcr/abl peptides.

These data clearly indicate that HA-1 is a target of GvL activity during GvHD as well as without GvHD. The immunodominant mHAg HA-1 is exclusively expressed on cells of hematopoietic origin including leukemia and tumor cells and does not induce GvHD in a skin explant model. HA-1 may be used as peptide vaccine or as immunotherapy with HA-1-loaded dendritic cells or HA-1-specific T cells.

\section{P506 \\ Pre-Emptive Therapie with Defibrotide to Prevent Severe Veno-Occlusive Disease of the Liver after Stem Cell Transplantation}

v. Hinüber $R .{ }^{1}$, Kröger $N^{2}{ }^{2}$, Zabelina $T^{2}{ }^{2}$, Hassenpflug $W^{3}$, Panse J. ${ }^{4}$, Born O. ${ }^{5}$, Görtitz I. ${ }^{3}$, Ayuk F. ${ }^{2}$, Schieder . $^{2}$, Fehse N. ${ }^{2}$, Kabisch $H^{2}{ }^{2}$, Schneppenheim . $^{2}$, Zander $A{ }^{1}$

${ }^{1}$ Children's University Hospital Luebeck - Schleswig-Holstein, Germany, ${ }^{2}$ Bone Marrow Transplantation, University Hospital, Hamburg, Germany, ${ }^{3}$ Pediatric Hematology \& Oncology, Children's University Hospital, Hamburg-Eppendorf, Germany, ${ }^{4}$ Internal Medicine, University Hospital, Hamburg, Germany, ${ }^{5}$ General Hospital Hamburg-Wandsbek, Surgery, Germany

Purpose: Hepatic veno-occlusive disease (VOD) occurs in 0-70\% of the patients, undergoing haematopoietic stem cell transplantation and carries a mortality rate of up to $67 \%$. Defibrotide (DF) is an effective treatment of established VOD. We explored if early intervention with DF might prevent severe VOD after stem cell transplantation. Methods: We report a non- 
randomised prospective analysis of defibrotide as "pre-emptive therapy" in 100 treatment cases and 90 patients with signs of liver toxicity after stem cell transplantation between October 1999 and January 2004. Pre-emptive DF was initiated with a bilirubin-increase above $3,0 \mathrm{mg} / \mathrm{dl}$, independent of other criteria. Bleeding was correlated to the bleeding score according to Nevo (Blood 91: 1469-1477, 1998). Median age was 31 years (range 0-65 y), 25 were younger than 18 years and 12 of them even younger then 10 years. Diagnosis were haematological malignancies $(\mathrm{CML}=27, \mathrm{AML}=20, \mathrm{ALL}=16$, $\mathrm{MM}=7, \mathrm{MDS}=7$ and others $\mathrm{n}=23$ ). $42 \%$ received sibling and $58 \%$ unrelated stem cells. Defibrotide was given intravenously, doses ranged from 30 to 60 $\mathrm{mg} / \mathrm{kg} / \mathrm{d}$. Clinical diagnosis of VOD was defined by the Jones Criteria (bilirubin $>2,0 \mathrm{mg} / \mathrm{dl}$ and two of the following criteria: hepatomegaly (usually painful), ascites or weight gain greater than $5 \%$ above admission weight). Results: $55 \%$ had a complete response of bilirubin, $3 \%$ developed severe VOD under DF-therapy. In $45 \%$ we had to discontinue treatment because of increasing bilirubin or for attributable adverse events. In $43 \%$ bleeding episodes occurred (score II 26\%, score III 12\%, score IV 5\%) and in 4 case they stopped after discontinuation. The most important kind of severe bleedings were pulmonary (score III+IV 5\%) and cerebral haemorrhages (score IV $1 \%$ ). $57 \%$ of the 100 cases had bleeding events score $0+\mathrm{I}$. Mortality rate 100 days after SCT was $20 \%$. Conclusions: DF is an effective pre-emptive treatment to prevent severe VOD. Despite this positive outcome careful monitoring for bleeding is necessary.

\section{P507}

Results and Safety of Open or Stereotactic Brain Biopsies In Patients With Intracerbral Lesions after Allogeneic Hematopoietic Stemm Cell Transplantation

Schleuning M., Burlakova I., Kaltenhäuser J., Judith D., Baurmann H., Lörcher U., Schwerdtfeger R.

Center for Hematopoietic Cell Transplantation and Dept. of Radiology, German Diagnostic Clinic Foundation, Wiesbaden, Germany

Purpose: To date there is no standardized diagnostic approach in patient (pts) with intracerebral lesions who have undergone allogeneic hematopoietic stem cell transplantation. This study was undertaken to evaluate the safety and the diagnostic potential of invasive methods, i.e. open or stereotactic brain biopsies in comparison to less invasive methods, like CSF-analysis and MRI. Methods: The records and neuro-imaging studies of 6 pts with posttransplant CNS lesions in which diagnostic tissue has been obtained by open or stereotactic brain biopsy between 2002 and 2004 were reviewed retrospectively. Results: Five pts received an allotransplant from an unrelated dono and one from a HLA-identical sibling. The underlying diseases were AML $(n=4)$, OMF $(n=1)$ and CML $(n=1)$. At the time of biopsy the median patien age was $48.5 y$ (36-61y) and 2 of the 6 pts suffered from limited chronic GVHD requiring corticosteroid treatment. The median time from transplan to the onset of neurological symptoms was $247 \mathrm{~d}(120-1017 \mathrm{~d})$ and the interva between the onset of symptoms and biopsy was $27.5 \mathrm{~d}$ (9-36d). Four pts underwent open and 2 pts stereotactic biopsy. Besides the occurrence of postoperative seizures in 2 pts the procedure was well tolerated and there were no bleeding complications. The biopsy results are compared to CSF and MRI findings in table 1 . Three pts (nocardiosis, whipple disease, cavernoma) were successfully treated and are alive and well at a median of $75 \mathrm{~d}(63-835 \mathrm{~d})$ after biopsy. The pt with JC-PML died 49d after biopsy due to progressive PML, the pt with AML relapse 25d later from Aspergillosis after systemic chemotherapy and the pt with EBV lymphoma 4d after biopsy from sepsis. Conclusions: Open or stereotactic brain biopsies can safely be performed after allogeneic stem cell transplantation in pts with brain lesions of unknown origin and may uncover rather unexpected and possibly treatable conditions.

Table 1:

\begin{tabular}{|l|l|l|l|l|l|l|}
\hline & \multicolumn{1}{|c|}{$\# 1$ 1 } & \multicolumn{1}{|c|}{$\# 2$} & \multicolumn{1}{|c|}{$\# 3$} & \multicolumn{1}{|c|}{$\# 4$} & \multicolumn{1}{|c|}{ 5 } & n \\
\hline CSF & normal & normal & normal & n.d. & $\begin{array}{l}\text { normal } \\
\text { pleocytosis, } \\
\text { EBV-PCR pos }\end{array}$ \\
\hline $\begin{array}{l}\text { MRI } \\
\text { sugge- } \\
\text { stive of })\end{array}$ & $\begin{array}{l}\text { Asper- } \\
\text { gillosis }\end{array}$ & $\begin{array}{l}\text { Toxoplas- } \\
\text { mosis }\end{array}$ & $\begin{array}{l}\text { Toxoplas- } \\
\text { mosis }\end{array}$ & $\begin{array}{l}\text { Lym- } \\
\text { phoma }\end{array}$ & $\begin{array}{l}\text { Toxoplas- } \\
\text { mosis }\end{array}$ & $\begin{array}{l}\text { Aspergill- } \\
\text { osis }\end{array}$ \\
\hline $\begin{array}{l}\text { Biopsy } \\
\text { result }\end{array}$ & $\begin{array}{l}\text { Nocar- } \\
\text { dia }\end{array}$ & JC-PML & $\begin{array}{l}\text { Tropheryma } \\
\text { whippelii }\end{array}$ & $\begin{array}{l}\text { Localized } \\
\text { AML- } \\
\text { relapse }\end{array}$ & $\begin{array}{l}\text { Caver- } \\
\text { noma }\end{array}$ & $\begin{array}{l}\text { EBV- } \\
\text { Lymphoma }\end{array}$ \\
\hline
\end{tabular}

P508

The Impact Of Donor Type on the Survival of Patients with CLL, who Received Reduced-Intensity Conditioning and Allogeneic Stem Cell Transplantation

\author{
Schetelig J. ', Bornhäuser M., Geissler G., Thiede C., \\ Oelschlägel U., Mohr B., Schwerdtfeger R., Kiehl M., Kröger N., \\ Kienast J., Hänel M., Ehninger G., Siegert W. on behalf of the \\ Cooperative German Transplant Study Group \\ 'Uniklinikum Carl Gustav Carus, TU Dresden
}

Purpose: To study the impact of the donor type (related versus unrelated) for patients with chronic lymphocytic leukemia (CLL) who receive hematopoietic stem cell transplantation (HSCT) after reduced-intensity conditioning. Methods: 60 patients with advanced CLL were included. The median age was 52 years (range 12-68). A median number of 3 chemotherapy regimens had been given before HSCT. After conditioning with fludarabine, busulfan and ATG $(n=40)$ or Campath $(n=20)$ patients received HSCT from related $(23$ patients) or unrelated donors (37 patients). Graft-versus-host disease (GVHD) prophylaxis consisted of cyclosporine alone or a combination with methotrexate or mycophenolate mofetil. Results: After a median follow-up period of 32 months (range 2-70), 39 patients are alive. Acute GVHD Grade II-IV occurred in $26 \%$ of patients with related compared to $70 \%$ of patients with unrelated donors $(\mathrm{p}=0.007)$. Extensive chronic GVHD occurred in $9 \%$ and $25 \%$ of patients $(\mathrm{p}=0.063)$, respectively. At three years the probability of overall survival was $62 \%(95 \% \mathrm{CI}, 48 \%$ to $86 \%)$ for patients with related donors and $57 \%$ (95\% CI, $39 \%$ to $75 \%$ ) for patients with unrelated donors (log-rank, $\mathrm{p}=0.3267)$. The 3-year probability of progression-free survival was $52 \%(95 \%$ CI, $30 \%$ to $74 \%)$ for patients with related donors and $51 \%(95 \%$ CI, $33 \%$ to $69 \%$ ) for patients with unrelated donors (log-rank, $\mathrm{p}=0.8366$ ). Relapse mortality was $32 \%(95 \%$ CI, $8 \%$ to $56 \%$ ) for patients with related donors and $8 \%(95 \% \mathrm{CI}, 0 \%$ to $20 \%$ ) for patients with unrelated donors (logrank, $\mathrm{p}=0.2354)$, and the probability of non-relapse mortality was $9 \%(95 \%$ CI, $0 \%$ to $21 \%$ ) for patients with related donors and $37 \%$ (95\% CI, $19 \%$ to $55 \%$ ) for patients with unrelated donors (log-rank, $\mathrm{p}=0.0393$ ). Conclusions: Overall and progression-free survival of patients with advanced CLL after allogeneic stem cell transplantation did not differ with respect to the type of donor, although causes of death differed largely. Searching for unrelated donors is therefore suggested in patients with advanced CLL who do not have a family donor. Future transplant strategies should focus on improved disease control in patients with family donors and on further reduction of treatment related mortality in patients with unrelated donors.

\section{P509}

\section{Expression of HA-1 and HA-2 in Patients with Haematological Malignancies and in their HLA-Matched Stem Cell Donors: A Retrospective Analysis}

\section{Tschiedel S. ${ }^{1}$, von Rundstedt F.C. ${ }^{1}$, Reinhardt A. ${ }^{1}$, Pool J. ${ }^{2}$,} Goulmy E. ${ }^{2}$, Niederwieser D. ${ }^{1}$, Gentilini C.

${ }^{1}$ University of Leipzig, Division of Hematology and Oncology, Leipzig, Germany, ${ }^{2}$ Dept. of Immunohematology and Blood Transfusion, Leiden University, The Netherlands

Purpose: Minor histocompatibility antigens (mHAs) are peptides derived from polymorphic proteins, which can be responsible for graft versus host disease (GvHD) as well as for graft versus leukaemia $(\mathrm{GvL})$ reactions in patients after allogenic stem cell transplantation. Immune responses against mHAs with broad tissue expression can result in a high incidence of GvHD, whereas mHAs with restricted tissue distribution seem to be ideal candidates for effective GVL reactions. In order to estimate the applicability of mHAs specific immunotherapies after stem cell transplantation, we analyzed the frequencies of allelic mismatches between recipients and donors, with regard to the HLA-A2 restricted mHAs HA-1 and HA-2. Both mHAs are expressed by hematopoietic cells. HA-1 has also been described on several types of solid tumors, including breast and renal cancer. There are two allelic counterparts, HA-1H and HA-1R and HA-2V and HA-2M, respectively. The HA-1H and HA-2V alleles are the immunogenic variants, which can elicit $\mathrm{T}$ cel responses after stem cell transplantation. Methods: In our analysis 62 HLAA2+ patients with different hematological malignancies were included. These patients underwent allogenic stem cell transplantation with reduced conditioning. Using an allele specific PCR for HA-1 and HA-2 we performed a 
genomic typing of all donor/recipient pairs. Results: $56 \%$ of the analyzed subjects were positive for the HA-1H allele, therefrom were 35\% homozygote and $21 \%$ heterozygote. Furthermore, $44 \%$ of the population tested were homozygote for HA-1R. The ideal donor/recipient combination (donor RR, recipient $\mathrm{HH}$ or $\mathrm{HR}$ ) was found in 14 pairs $(23 \%)$. When the HA-2 allele was analyzed, $92 \%$ of this population expressed the V allele (52\% HA-2VV, $40 \%$ HA-2MV). The ideal combination (donor MM, recipient VV or MV) was seen in only 2 pairs (3\%). Conclusions: Since approximately $25 \%$ of the analyzed donor/recipient pairs show a mismatch in the HA-1 locus, this mHA could be a useful tool in the treatment of haematological malignancies relapsing after stem cell transplantation. In contrast, allelic mismatches in the HA-2 locus are present in less than $5 \%$ of the couples so that this antigen does not seem a useful tool for immunotherapy in most cases of transplantation between HLA-identical individuals.

\section{P510}

\section{Mycophenolate Mofetil AUC Targeting for GvHD Prophylaxis after Allogeneic Hematopoetic Stem Cell Transplantation}

Freiberg-Richter J., Jenke A., Pursche S., Bonin M., Schleyer E., Ehninger G., Bornhäuser M.

Universitätsklinikum Dresden, Germany

Purpose: Mycophenolate mofetil (MMF) is an effective immunosuppressive drug and well established to prevent graft rejection in solid organ transplantation. Studies in recipients of solid organ grafts have shown that MPA trough plasma levels are not sufficient to predict the effectiveness of therapy. Further investigations provided some evidence that a MPA-AUC0-12 (area under concentration-time curve) between $30-60 \mu \mathrm{g} / \mathrm{ml} * \mathrm{~h}$ are necessary for effective immunosuppression in renal transplantation. In contrast, studies by our group demonstrate low MPA plasma levels in the early phase after conditioning therapy and HCT. Most likely as a result of this a high rate of acute and hyperacute GvHD was observed. Methods: We launched a study for patients with MDS or AML receiving conditioning with fludarabine and busulfan and hematopoetic stem cells from HLA-compatible siblings or unrelated donors using a combination of tacrolimus and MPA-AUC targeted iv MMF as GvHD prophylaxis. The target range of MPA-AUC0-12 was $30-45 \mu \mathrm{g} / \mathrm{ml} * \mathrm{~h}$. $12 \mathrm{~h}$ AUC measurements were performed once a week on three occasions. For detection of MMF and MPA we used an isocratic RP-HPLC-system with Fluorescence-Spectroscopy. MMF doses were adjusted in steps of $500 \mathrm{mg} / \mathrm{d}$ calculated by taking into account the relative difference between measured and target MPA-AUC0-12. Results: Preliminary data in six patients indicate that targeted dose adjustment (up to $4.5 \mathrm{~g} / \mathrm{d}$ ) is feasible to achieve therapeutic MPA-AUC (median MPA-AUC0-12 after second dose adjustment = $\left.33 \mu \mathrm{g} / \mathrm{ml}{ }^{*} \mathrm{~h}\right)$ and is well tolerated by most of the pts $(2 / 6$ pts mild gastrointestinal pain). Acute extramedullary toxicity seems to be less compared to standard prophylaxis with CSA/MTX. In the evaluated patients the rate of acute GvHD is comparable to standard regimen (2 pts. with grade II GvHD). Conclusions: MMF adjusted according to MPA-AUC seems to be a feasible way to achieve therapeutic drug levels, which might result in effective contro of GvHD and are tolerated well by the patients. Further accrual and follow-up is necessary to confirm the efficacy of this approach.

\section{P511}

\section{Clobazame (CLB) for the Prevention of Seizures Associated with High-Dose Oral and Intraveneous Busulfan (HDBU) in Patients Receiving an Allogeneic Hematopoietic Stem Cell Graft (alloHSCT)}

\section{Schwerdtfeger R., Schleuning M., Judith D., Yaman A., Kaltenhäuser J., Baurmann $H$. \\ Zentrum für Blutstammzell- und Knochemarktransplantation,} Deutsche Klinik für Diagnostik, Wiesbaden, Germany

Background: Conditioning with HDBU requires prophylactic anticonvulsan therapy. Phenytoin (DPH) is an effective and widely used agent for this purpose. It is however associated with adverse reactions in $1 / 3$ of patient (pts), some of them severe. Daily monitoring of blood DPH concentration is mandatory to ensure therapeutic levels. DPH induces an increased clearance of BU and Cyclophosphamide (CY) from the blood and thereby may influence transplant results. Clobazame (CLB), a 1,5-benzodiazepine effective in treating and preventing seizures has no significant side effects or interaction with other drugs. After oral administration it is well absorbed and well tolerated. Here we review our experience using exclusively CLB for seizure prophylaxis in 177 adults receiving HDBU orally $(n=144)$ and intraveneously $(\mathrm{n}=33)$ as part of their preparative regimen for alloHSCT. Patients and methods: 177 pts $(\mathrm{m} / \mathrm{f}=103 / 74)$, median age $38 \mathrm{y}(16-65)$ were treated with $16 \mathrm{mg} / \mathrm{kg}$ bw BU po $(1 \mathrm{mg} / \mathrm{kg}$ q $6 \mathrm{hrs}$ for 4 days $)$ and $12.8 \mathrm{mg} / \mathrm{kg}(\mathrm{n}=27)$ or $9.6 \mathrm{mg} / \mathrm{kg}$ bw (n=6) BU iv (16 and 12 doses of $0.8 \mathrm{mg} / \mathrm{kg} \mathrm{q} 6 \mathrm{hrs}$ ) resp followed by CY $(120$ or $200 \mathrm{mg} / \mathrm{kg})(\mathrm{n}=166)$ or Cy plus VP16 $(\mathrm{n}=11) .83 \mathrm{pts}$ received a graft from a matched related and 94 from an unrelated donor Median body weight was $72 \mathrm{~kg}$ (49-110). Indication for HSCT was AML (50), ALL (2), MDS (12), CML (109), OMF (3), PNH (1). In all pts CLB was started the day before the first BU dose and continued for the follwing 9 days. The exact protocol was as follows: CLB 5, 5, 10mg (i.e. three doses each day) starting from day before the first $\mathrm{BU}$ dose through the $1^{\text {st }}$ day after the last BU dose, $5,5,10 \mathrm{mg} /$ day on the $2^{\text {nd }}, 0,0,10 \mathrm{mg} /$ day on the $3^{\text {rd }}$ and $0,0,5$ $\mathrm{mg} /$ day on the $4^{\text {th }}$ and $5^{\text {th }}$ day after the last BU dose. Antiemetic prophylaxis consisted of a 5 HT antagonist. Results: All 177 pts received the CLB dose prescribed. CLB was well tolerated. Somnolence was absent or minimal. No serious side effects like respiratory depression or sedation attributable to CLB were observed. No generalized seizure or any other sign of neurotoxicity were seen during or after HDBU. Conclusion: In pts receiving HDBU as conditioning for alloHSCT CLB safely prevents seizures. Using the described protocol clinically effective CLB plasma levels are acchieved with no need for expensive and time consuming drug monitoring like in DPH prophylaxis. Anticonvulsive prophylaxis with CLB is therefore easy to perform, well tolerated and cost effective.

\section{P512}

\section{Differential Sensitivity of Primary Leukemia Blasts to Perforin/Granzyme B}

\section{Grüllich C., Friske V., Finke J.}

University Hospital Freiburg, Germany

Purpose: CTL induced apoptosis in leukemic blasts is the molecular mechanism involved in the Graft versus leukemia $(\mathrm{GvL})$ reaction following allogenic bone marrow (BMT) and peripheral stem cell transplantation (PBSCT) This effect is being taken advantage of by using donor lymphocyte infusion (DLI) following post transplant relapse. Many groups have reported remission induction by DLI. However, many patients do not respond to DLI or become refractory. Thus, it is important to further identify mechanisms of resistance to CTL induced apoptosis. We have investigated the sensitivity of primary leukemic cells to the main molecular effectors of CTL for induction of apoptosis: the pore forming protein perforin and the serine protease granzyme B. Methods: Cytotoxic Granules rich in perforin/granzyme B were purified using the human NK cell line YT. Briefly, cells were lysed by hypotonic swelling and granules were isolated by sequential centrifugation to remove nuclei, heavy membrane and mitochondria fractions. Lysates were tested for apoptosis induction in HeLa cells. Granzyme B uptake was measured by western blot. Death receptor caspase pathway induced apoptosis was excluded using caspase inhibitors IETD and ZVAD. Cell lines and primary cells were incubated with granules and membrane permeabilization was measured after 4 hours by PI uptake using a FACS Calibur FACSscan Results: Primary cells from 21 patients were investigated with AML $(n=8)$, $\operatorname{ALL}(n=6)$ and CLL $(n=7)$. We found strong interindividual variation in sensitivity to perforin. Between different entities a trend could be shown towards weaker responses in AML cells, intermediate responses in ALL cells and the strongest responses in CLL cells. Conclusions: We could show that leukemic cells show differential sensitivities to purified cytotoxic granule proteins perforin/granzyme B. Thus, resistance mechanisms at this level of apoptosis induction by CTL must exist. It remains to be further investigated how sensitivity to perforin/granzyme B correlates to clinical outcome following allogenic BMT/PBSCT and DLI after relapse. 
P513

Pharmakokinetics of Oral Valganciclovir versus i.v. Ganciclovir for Allogeneic Stem Cell Transplantation

Einsele H. ${ }^{1}$, Reusser $P^{2}{ }^{2}$, Hebart $H^{1}{ }^{1}$, Hertenstein $B .{ }^{3}$, Bornhäuser $M_{.}{ }^{4}$, Ehninger $G .{ }^{4}$, Kröger ${ }^{5}$. , Kalhs $P .{ }^{6}$, Chalandon $Y^{7}$, Rohde $F^{8}$

${ }^{1}$ Univ. Tübingen, Germany, ${ }^{2}$ Univ. Basel, Switzerland, ${ }^{3}$ Univ. Hannover, Germany, ${ }^{4}$ Univ. Dresden, Germany, ${ }^{5}$ Univ. Hamburg,

Germany, ${ }^{6}$ Univ. Wien, Austria, ${ }^{7}$ Univ. Genève, Switzerland,

${ }^{8}$ Hoffmann-La Roche AG, Grenzach-Wyhlen, Germany

Background: After alloSCT, oral VALGCV is a promising alternative to IV GCV against CMV but its pharmacokinetics (PK) have not been studied. Methods: We investigated the PK of GCV after oral VALGCV in a randomized, crossover phase II-study of 48 patients (pts) after alloSCT. Pts who had $>400$ copies/ml of CMV DNA in plasma by quantitative PCR were randomized to receive oral VALGCV $900 \mathrm{mg}$ BID for 7 days, followed by IV GCV as 1 -h-infusion at $5 \mathrm{mg} / \mathrm{kg}$ every $12 \mathrm{~h}$ for another 7 days, or to the inverse sequence of study drug administration. The PK of GCV were assessed on days 4 and 11. Safety monitoring was done until day 84 after SCT. Results: 28 pts were fully assessable for PK analyses. Among the 22 pts without intestinal graft-versus-host disease (GVHD), the mean \pm SD AUC0-12 $(\mathrm{mg} / \mathrm{L} * \mathrm{~h})$ was $53.8 \pm 18.0$ on oral VALGCV vs $39.5 \pm 13.9$ on IV GCV (mean difference 14.3 [95\% CI 7.6 to 20.9 ]); $\mathrm{Cmax}(\mathrm{mg} / \mathrm{L}$ ) was $8.8 \pm 2.4$ vs $10.3 \pm 2.1$; tmax (h) was $2.7 \pm 0.8$ vs $1.1 \pm 0.6$; and $t 1 / 2$ (h) was $4.2 \pm 1.1$ vs $3.4 \pm 0.8$. Among the $6 \mathrm{pts}$ with gut GVHD, the mean \pm SD AUC0 $-12(\mathrm{mg} / \mathrm{L} * \mathrm{~h})$ was $46.6 \pm 24.9$ on oral VALGCV vs $35.3 \pm 12.8$ on IV GCV (mean difference 11.3 [95\% CI -13.4 to 35.9]); $\mathrm{Cmax}(\mathrm{mg} / \mathrm{L}$ ) was $7.1 \pm 3.6$ vs $11.1 \pm 3.1$; tmax (h) was $2.7 \pm 0.8$ vs $1.2 \pm 0.4$; and $\mathrm{t} 1 / 2(\mathrm{~h})$ was $5.6 \pm 2.0$ vs $3.3 \pm 0.7$. Clearance of viral DNA was equally effective in both arms. Nonfatal CMV pneumonia developed in $2 \mathrm{pts}$ during follow-up after antiviral therapy, and neutropenia $<500 / \mu 1$ occurred in 3 pts on IV GCV. Conclusions: After alloSCT, systemic exposure to GCV with oral VALGCV treatment is superior to that with IV GCV in pts withou gut GVHD, and is within the range seen with IV GCV in pts with gut GVHD. Oral VALGCV could replace preemptive IV GCV after alloSCT if shown similarly efficacious.

\section{P514}

\section{Immuncytological and -Histological Characterization of Donor Lymphocytes Infiltrating the Gastrointestinal Tract of Patients with GvHD}

Rieger K., Fietz T., Sommer D., Müßig A., Blau I.W., Knauf W., Thiel E., Uharek L.

Department of Hematology, Oncology and Transfusion medicine, Charité-Campus Benjamin Franklin, University School of Medicine Berlin, Germany

Purpose: Intestinal $\mathrm{T}$ cells have a unique state of activation and differentiation which might specifically affect or be affected by intestinal GvHD. While peripheral blood lymphocyte-subsets are well characerized after allogeneic stem cell transplantation (SCT), little is known about the infiltration of the gastrointestinal (GI) tract with donor-derived lymphocytes and about lymphocyte-subpopulations in GI-epithelia in GvHD. We therefore developed a method to isolate lamina propria and epithelium lymphocytes directly from GI-biopsies. Methods: Biopsies of small or large intestine were preincubated for 10 hours $\left(4^{\circ} \mathrm{C}\right)$ in collagenase-containing medium and then incubated for 4 hours $\left(37^{\circ} \mathrm{C}\right)$ in a humid chamber. GI-lymphocytes were then separated from epithelial cells by Ficoll-gradient centrifugation. Cells were stained for CD3, CD4, CD8, CD2, CD56, CD45R0, and CD25 and then analysed by three colour flow cytometry. Results: In 13 patients, lymphocytes were isolated from intestinal biopsies with a viability of $>90 \%$. In six patients without GvHD and in healthy controls, GI-lymphocyte-subsets correlated with proportions in peripheral blood, with a CD4+/CD8+-T cell-ratio of approximately 1:1. In contrast, GI-lymphocytes derived from patients with intestinal GvHD showed no correlation to peripheral blood subsets. GITderived lymphocytes of patients with histological confirmed intestinal GvHD were dominated by $\mathrm{CD} 3+/ \mathrm{CD} 8+/ \mathrm{CD} 45 \mathrm{R} 0+\mathrm{T}$-lymphocytes (median $77 \%$, range 62-87), while $\mathrm{CD} 3+/ \mathrm{CD} 4+$ lymphocytes were dramatically reduced to a median of $2 \%$ of $\mathrm{T}$ cells (range 1-30). The results were confirmed by immunhistochemical staining (CD4, CD8) of 18 paraffin-embedded sections derived from a different series of patients. The IL-2 receptor (CD25), which is the target of therapeutical anti-IL2 MoAbs, was expressed on a median of $14 \%$ (range 6-26) of the GI-lymphocytes in patients with GvHD, while samples of transplanted patients without GI-GvHD had a median of $8 \%$ CD25+ T cells (range 3-10). After treatment with the anti-CD25 monoclonal antibody Basiliximab, CD25 positive lamina propria lymphocytes disappeared completely, while $30 \%$ of peripheral blood lymphocytes still expressed CD25. Conclusion: The analysis of lymphocyte-subpopulations isolated from the GI tract improves our understanding concerning the repopulation of the GI tract after allogeneic stem cell transplantation and of the mechanisms leading to GI-GvHD.

\section{P515 \\ Haploidentical Peripheral Blood Stem Cell Transplantation: A Single Centre Experience of 24 Cases}

\section{Ringhoffer M. ${ }^{1}$, Wiesneth M. ${ }^{2}$, v. Harsdorf S. ${ }^{1}$, Zenz $T^{1}{ }^{1}$ Schlenk R. ${ }^{1}$, Duncker C. ${ }^{1}$, Grimminger W. ${ }^{1}$, Röttinger E. $^{3}$ Reske S.N. ${ }^{4}$, Döhner H. ${ }^{1}$, Bunjes D. ${ }^{1}$ \\ ${ }^{1}$ Dept. of Internal Medicine III, ${ }^{2}$ Dept. of Transfusion Medicine, ${ }^{3}$ Dept. of Radiation Therapy, ${ }^{4}$ Dept. of Nuclear Medicine, University Hospital Ulm, Germany}

Purpose: Over the past 8 years we have performed 24 haploidentical peripheral blood stem cell transplants in patients (pts)with incurable malignancies and no prospect of a matched unrelated donor within adequate time period. Patients and methods: We treated 12 females and 12 males with a median age of 37 years (range $20-57$ years). The diagnoses and remission status were as follows: AML not in CR $(n=9)$, AML in CR2 $(n=4), C M L>C P 1$ $(\mathrm{n}=7)$, CML in CP1 $(n=3)$ and ALL, Philadelphia chromosome positive in CR1 $(n=1)$. Conditioning consisted of ATG, TBI, thiotepa, cyclophosphamide in most patients and additional radioimmunotherapy with an Yttrium-90 or a Rhenium-188 labelled anti-CD66 antibody was given in 15 patients. All patients received G-CSF moblized peripheral blood stem cell grafts. GVHD prohylaxis consisted of T cell depletion by CD34+ selection in all but one pt, no posttransplant immunosuppression was given in 21 pts. Results: Stable engraftment was achieved in 23 pts. 1 case of acute graft rejection was observed. 11 patients developed grade I-II acute GVHD, we observed no cases of grade III-IV disease. 11 pts have developed chronic GVHD. 5 pts have suffered a relapse of their disease, 12 pts died of transplant related mortality. Infections were the most significant problem after transplant, in particular aspergillus infections. After a median follow-up of 39 month (2-59 months) 7 pts are surving in hematological remission, 1 pt with CML has minimal residual disease and is treated with STI-571. Conclusion: Haploidentical peripheral blood stem cell transplantation is a possible alternative to a MUD transplant. Maintenance of the infection prophylaxes for a prolonged period of time and frequent screening for infections are mandatory to reduce transplant-related mortality.

P516

\section{Rituximab-Monotherapy in Patients with Follicular Lymphoma Relapsing after ASCT}

Krieger O., Greinix H., Kasparu H., Mitterbauer M., Jäger, U., Lutz U. ${ }^{1}$ 1st.Int. Dept., Kh Elisabethinen, Linz, Austria, ${ }^{2}$ Int.1 Dept \& BMT, AKH, Vienna, Austria

The prognosis of patients with follicular lymphoma (FCL), relapsing after autologous stemcell-transplantation (ASCT) is poor. These patients have chemo-resistant disease and sometimes limited bone marrow capacity, therefore antibody treatment may afford an alternative option.

We initiated a multicenter phase IV trial with rituximab for patients with CD 20 pos. FCL, relapsing more than 6 months after ASCT. Initial post-transplant treatment-regimen consisted of rituximab $\left(375 \mathrm{mg} / \mathrm{m}^{\wedge} 2\right)$ weekly. After completion of fourth cycle patients entered an observation period. Results: 12 patients ( 9 male, 3 female ), 28-61 years old (median: 48,5) were treated. Median time from diagnosis to ASCT was 30 months (range: 9-65) and median time from ASCT to rituximab 18 months (range 6-52). Treatment was well tolerated, in 4/48 therapy cycles grade III-IV toxicity ( 2 FUO, 1 bronchopulmonary infection, 1 chills+pain ) was observed only. No delayed granulocytopenia occurred. Responses were achieved in $8 / 12(66 \%)$ patients - 5 $\mathrm{CR}, 3 \mathrm{PR}-, 4$ patients were refractory to rituximab (NC): $2 / 4 \mathrm{NC}$ died within 1 month, one patient was treated with chemotherapy and lives in PR for 23 months; 1 NC-patient was transplanted (RIC-alloSCT) and is alive in CCR 
for 54 months. 3/8 responders relapsed and died after 20, 43 and 44 months despite chemotherapy. 5 patients are alive ( 4 in CCR -2 after RIC-alloSCT, 1 in PR) 18, 24, 29 and 46 months after initiating rituximab. Conclusion: Rituximab is an effective and safe therapy for patients with FCL relapsing after ASCT with a $66 \%$ response rate. But to achieve a continuous complete remission additional chemotherapy or RIC-alloSCT was needed in one third of our patients.

\section{P517}

High Dose Chemotherapy And Autologous Stem Cell Transplantation For Relapsed Chemosensitive Hodgkin's Disease - A Single Center Experience

\section{Zingerle M., Goretzki M., Fischer N., Nerl C.}

1. Medizinische Abteilung, Städtisches Krankenhaus-Schwabing, München, Germany

Backround: High dose chemotherapy (HDC) followed by autologous stem cell transplantation represents an effective treatment modality in relapsed Hodgkin's disease (HD). We evaluated efficacy and toxicitiy of this therapy in patients (pts) with relapsed $\mathrm{HD}$, who were treated in our institution between 1997 and 2003. Patients and Methods: 18 pts. were treated within this period, median age at transplantation was 34 yrs. (range, 19-61). 16 pts. were treated in first relapse, median time to relapse was 33 months (range, 2 95). 2 pts. had a primarily resistant disease. As induction chemotherapy pts received different regimens including Dexa-BEAM, High-Dose (HD)-Etoposide, HD-Cyclophasphamide, HD-Methotrexat, DHAP, IEV. All patient achieving at least a PR proceeded to transplantation (100\%). The most frequently used high-dose regimen was BEAM. In 2 pts. with early relapse and 1 pts. with resistant disease during the first induction regimen a double transplantation with TCM and BEAM was performed. Median time from relapse to transplantation was 5 months (range, 2-20). Results: Median follow up was 42 months (range, 5-82). 17 of 18 (94\%) transplanted pts achieved CR, 1 pt. who was primarily resistant achieved PR. 2 (11\%) pts relapsed at 20 and 59 months. Disease free survival at 3 years was $89 \%$, overall survival at 3 years were $100 \%$. Out of 11 pts. with a follow up more than 3 yrs., only 1 pt. relapsed after 5,9 yrs. and died from second relapse. Transplantation related toxicity was as expected: $67 \%(\mathrm{n}=11)$ suffered $>$ grade II and $27 \%(n=5)>$ grade III toxcicity. 1 pt. developed aspergillus pneumonia and 1 pt. thrombosis of V. cephalica. There was no transplantrelated death. Conclusion: Our data confirm that High Dose Chemotherapy with autologous stem cell transplantation is associated with a high response rate resulting in durable disease free survival. Transplantation associated toxicity was acceptable

\section{P518}

\section{Autologous DC Transfected with in Vitro Transcribed mRNA for the Analysis of CMV-pp65-Specific T Cell} Reconstitution after Allogeneic Stem Cell Transplantation

\section{Meyer R.G., Britten C.M., Gstöttner A., Huber C., Wölfel T.,} Herr W.

3rd Departement of Internal Medicine, University Hospital Mainz, Germany

Purpose: CMV-specific T cell reconstitution after allogeneic stem cell transplantation has been examined by several investigators. Most analyses used known peptide epitopes derived from CMV-proteins (mainly of pp65) to examine the frequency of $\mathrm{CMV}$-specific $\mathrm{CD}^{+} \mathrm{T}$ cells with either HLA/peptide multimers in flow cytometry or with peptide loaded antigen presenting cells (APC) in cytokine ELISPOT assays. The use of peptides, however, is limited to known epitopes and to the presence of their restricting HLA-alleles. We have previously shown with peripheral blood lymphocytes (PBL) of healthy donors, that RNA-transfected autologous dendritic cells (DC) can be applied to detect CMVpp65-specific T cells in IFN-gamma ELISPOT assays. We have also demonstrated that the sensitivity of this approach is similar to the use of peptide loaded APC. Methods: Here we report on four patients who underwent allogeneic stem cell transplantation for haematological malignancies. All patients and their donors were CMV-positive and experienced at least two CMV-reactivations. PBL were analyzed prior to, and at least at two timepoints after transplantation. For two HLA-B7positive patients the immunodominant HLA-B7-restricted pp65-peptide
TPRVTGGGA (pp65 $404-412$ ) was used as a positive control. Results: Specific $\mathrm{CD}^{+} \mathrm{T}$-lymphocytes pre-transplant ranged between 0.15 and $1.0 \%$ of peripheral blood $\mathrm{CD}^{+}$cells. Using pp65-RNA transfected autologous DC increasing frequencies of pp65-specific $\mathrm{CD}^{+} \mathrm{T}$-cells were detected after transplantation. The course of CMV-specific reconstitution differed significantly between individual patients. In both HLA-B7-positive patients pp $65_{404}$

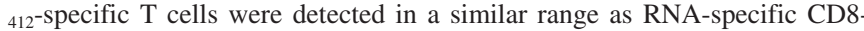
positive cells, underlining the comparable sensitivity of this approach. In three of four patients CD4-positive cells specifically recognizing pp65-RNA transfected autologous DC were also detected. The frequencies of specific $\mathrm{CD}^{+} \mathrm{T}$-cells ranged between 14 and $80 \%$ of those of specific CD8 $8^{+}$lymphocytes. Conclusions: Our data indicate the feasibility of using RNA-transfected autologous DC as antigen-presenting cells in IFN-gamma ELISPOT assays for the sensitive and HLA-independent monitoring of CMV-specific $\mathrm{CD}^{+}$and $\mathrm{CD}^{+}$immune reconstitution. RNA-transfected DC might be also used for other viruses, such as HSV, RSV and adenovirus, where immunodominant epitopes have been poorly described.

\section{P519 \\ Every-Other-Day HPC-Collection Improves CD34+ Cell Counts in Poor Mobilizers}

Reinhardt P., Schauwecker P., Maccari B., Schrezenmeier H., Wiesneth $M$.

Institute for Clinical Transfusion Medicine and Immunogenetics Ulm, Department of Transfusion Medicine, University of UIm, Germany

Purpose: To collect satisfying autologous transplants in poor mobilizing patients. Repeated aphereses will be required and patients will be exposed to excessive amounts of DMSO when receiving transplants with high nucleated cell but low hematopoetic progenitor cell (HPC) counts. To evaluate a different apheresis strategy, the CD34+ cell precounts of an every-day harves schedule were compared to those of an every-other-day collection regimen within the same patients. Methods: Thirteen patients suffering of advanced stages of hematologic malignancies had been treated with standard chemotherapy followed by G-CSF $5-10 \mathrm{mcg} / \mathrm{kg} / \mathrm{die}$ to allow for the collection of autologous transplants. For each patient the circulating HPC counts on every-day collections were compared to those following a one or two-day harvest free interval. For logistical reasons 5 out of these 13 patients had to return to an every-day apheresis rhythm. Peripheral HPC counts were evaluated by dual-platform analysis on a BD FACSort-analyser using the CELLQuest software and the analyzer Abbott CellDyn 3200. Results: Circulating CD34+ cell counts showed an average of $9.8+/-4.8 \mathrm{c} / \mathrm{mcl}$ before collection on day one and $9.5+/-5.3$ on day two before the following second collection. After a one or two day harvest free interval a statistically significant increase to $15.2+/-6.9$ circulating CD34+ $\mathrm{c} / \mathrm{mcl}$ was observed (Wilcoxon-rank $\mathrm{p}<0.01$ ). For logistical reasons 5 of these patients returned to an every-day apheresis schedule showing $6.5+/-2.9 \mathrm{CD} 34+\mathrm{c} / \mathrm{mcl}$ before collection interval, $13.5+/-6.7 \mathrm{CD} 34+\mathrm{c} / \mathrm{mcl}$ after the one-day collection interval (Wilcoxon-rank $\mathrm{p}<0.05$ ) and a significant drop to $8.5+/-4.0 \mathrm{CD} 34+$ $\mathrm{c} / \mathrm{mcl}$ on the following day after an every-day apheresis schedule was resumed (Wilcoxon-rank $\mathrm{p}<0.05$ ). Conclusions: There seems to be a slow release of CD34+ HPC in poorly mobilizing patients and an accumulation of HPC in the peripheral blood. An every-other-day harvest schedule leads to significantly higher CD34+ cell precounts and thus improved collection conditions. This benefit is not a consequence of prolonged mobilization since a return to an every-day apheresis strategy is paralleled by a drop in circulating $\mathrm{HPC}$.

\section{P520 \\ Donor Lymphocyte Infusions as a Successful Treatment of a Late Relapse of Chronic Idiopathic Myelofibrosis after Allogeneic Peripheral Blood Stem Cell Transplantation}

Schmalzin M., Faul C., Kanz L., Möhle R.

University Hospital Tübingen, Germany

Objectives: Donor lymphocyte infusions (DLI) constitute a well known therapeutic option for patients with relapsed CML following allogeneic stem cell transplantation. In contrast, only few patients with relapsed chronic idiopathic myelofibrosis (IMF) have been treated with DLI. Furthermore, there are no reports of IMF relapses receiving DLI later than 1 year after transplantation. 
A 45-year-old male patient with chronic idiopathic myelofibrosis and consecutive anemia, leukopenia and thrombocytosis received an allogeneic peripheral blood stem cell transplantation from an HLA-identical female sibling after conditioning with TBI/cyclophosphamide. He had a rapid take of donor hematopoesis and only moderate complications, including CMV reactivation one month posttransplant and grade I graft versus host disease of the skin as late as four months after transplant which responded well to steroids. Four and a half years later, relapse was diagnosed with leucocytosis of $11.260 / \mu 1$ an elevated hemoglobin level of 20,4 g/dl, thrombocytosis of 616.000/ $\mu 1$ and a marked reduction in donor chimerism, resembling prefibrotic phase of myelofibrosis. Methods: The patient received two DLI with increasing cell dose $\left(1 \times 10^{7} \mathrm{CD} 3\right.$ + cells per $\mathrm{kg}$ and $3 \times 10^{7} \mathrm{CD} 3$ + cells per $\mathrm{kg}$ bw., respectively). Results: The patient developed no significant graft versus host disease $(\mathrm{GvHD})$ of the skin or the gut. DLI were stopped due to suspected GvHD disease of the liver. However, elevated liver enzymes decreased without immunosuppression. Within 5 months after the last DLI, donor hematopoesis was fully restored and white blood count, hemoglobin and platelets returned to normal. At present, the patient is alive and well withou further relapse or GvHD almost one year after his last DLI. Conclusion: This case report indicates that DLI can be an effective treatment for patients with a late relapse of IMF after allogeneic stem cell transplantation. Interestingly, a late relapse may resemble early prefibrotic stage of this disease.

\section{P521}

\section{Targeting of Once Daily Intravenous Busulfan as Part of Conditioning Therapy}

Jenke A., Freiberg-Richter J., Schuler U., Pursche S., Haake A., Lorenz P., Wauer S., Schleyer E., Ehninger G., Bornhäuser M. University Hospital Carl Gustav Carus, Dresden University of Technology, Dresden, Germany

Purpose: Busulfan (BU) administered orally in a total dosage of $16 \mathrm{mg} / \mathrm{kg}$ over 4 days is widely used as a component of preparative regimens for allogeneic blood stem cell transplantation (BSCT). The individual bioavailability after oral BU administration differs extremely among the patients and depends on multiple factors. Several reports indicated an advantage for an intravenous BU solution reducing the intrapatient variation of BU pharmacokinetics, while interpatient variations were still high. Assuming an influence of the overall exposure to BU on the outcome after BSCT, a therapeutic drug monitoring (TDM) of BU seemed to reduce the incidence of relapse or regimen related toxicity (RRT). In a previous study we showed that a TMD of oral $\mathrm{BU}$ is a feasible procedure in patients receiving BSCT to minimize the risk of RRT. To improve this time and labor intensive procedure we evaluated a TDM of intravenous BU in patients with myelodysplastic syndrome or acute myeloid leukemia with high risk of RRT undergoing BSCT. Methods: BU dosages were adjusted to achieve concentrations at steady state (Css) of $900 \pm 100 \mathrm{ng} / \mathrm{ml}$. All patients $(\mathrm{n}=8)$ received a dose reduced conditioning regimen consisting of targeted BU and fludarabin (FLU) intravenously (4 x $30 \mathrm{mg} / \mathrm{m}^{2}$ ). BU was administered intravenously as single daily dose on 4 consecutive days. The first dose of BU was fitted using ideal body weight BU plasma levels were measured by a HPLC method. Consecutive BU doses were adjusted according to the measured Css levels. Results: The result showed a broad interpatient variation of BU plasma levels after first dose Dose corrections after first dose up to $25 \%$ were needed to achieve the target plasma levels. Largely, no significant further dose adjustments were necessary. The correlation coefficient between dose corrections and resulted changes in plasma levels was in an excellent range. The RRT was low in all patients receiving the described conditioning regimen. Conclusions: The conditioning therapy with targeted intravenous BU as single daily doses in combination with FLU is a feasible procedure to minimize the variation of BU pharmacokinetics. A single dose adjustment after the first dose of BU is sufficient to achieve stable target levels for the whole conditioning therapy. Compared to the targeting of oral BU the expenditure of the procedure could be reduced remarkably. Prospective and randomized clinical studies are warranted to investigate the impact of BU dose adjustment on the outcome after BSCT.

\section{P522}

Immune Reconstitution after Transplantation of Allogeneic Highly Purified Peripheral Blood CD34+ Cells compared to the use of Alemtuzumab for in vivo T-cell Depletion

\section{Peceny R., Elmaagacli A.H., Franke M., Trenschel R., Ottinger H., Beelen D.W.}

University Hospital Essen, Germany

Purpose: Immune reconstitution was studied in 154 patients transplanted from May 1999 to April 2004 using either highly purified peripheral blood CD34+ cells without any other prophylactic immunosuppression (72) or in vivo T-cell depletion with $100 \mathrm{mg}$ (47) or recently $50 \mathrm{mg}$ (35) Alemtuzumab and cyclosporine A (CsA) for 3 months post transplant. Methods: All patients having received $50 \mathrm{mg}$ Alemtuzumab are too early to analyze. In very short term, there is no significant difference of the two dosages in regard to the important clinical end points including acute GvHD and to immune reconstitution. Nineteen patients with a follow up of at least half a year have received $100 \mathrm{mg}$ Alemtuzumab (group A). Seventeen patients have been transplanted using CD34 enriched grafts including all 8 non-CML patients with a sufficient follow up period (group B). These are compared amongst each other and with a cohort of 20 successive patients transplanted after myeloablative conditioning with a standard immunosuppression (CsA and short course methotrexate) in the year 2002 (group C). Absolute numbers of $\mathrm{CD} 3+\mathrm{CD} 4+\mathrm{RO}+, \mathrm{CD} 3+\mathrm{CD} 4+\mathrm{RA}+, \mathrm{CD} 3+\mathrm{CD} 8+, \mathrm{CD} 16 / 56+\mathrm{CD} 3-$ and CD19+ cells were measured in the peripheral blood by flowcytometry at 3 and 6 months and one year post transplant. Results: At 3 months numbers of CD8+ T-cells reached a median of $228 / \mu 1$ in group B and $349 / \mu 1$ in group C vs. $50 / \mu 1$ in group A $(\mathrm{p}<0.01)$. The difference in CD8+ T-cell recovery persisted at one year with median numbers of $274 / \mu 1$ in Group B and 392/ $\mu 1$ in group $\mathrm{C}$ vs. $48 / \mu 1$ in group $\mathrm{A}(\mathrm{p}<0.03)$. The recovery of naïve $\mathrm{CD} 4+\mathrm{RA}+$ T-helper cells is delayed in groups $\mathrm{A}$ and $\mathrm{B}$ with $1 / \mu 1$ and $1 / \mu 1 \mathrm{vs} .24 / \mu 1$ in group $\mathrm{C}$ at 3 months $(\mathrm{p}<0.01)$ loosing significance thereafter $(\mathrm{p}=0.13$ at 1 year). CD19+ B-cells did recover up to 3 months in group A seldomly with a median of $3 / \mu 1$ and only slowly in group $C$ with a median of $21 / \mu 1$, but reached a median of $241 / \mu 1$ in group $B(p<0.001)$. This difference in B-cell recovery remained up to a year post transplant, group B showing higher Bcell numbers with a median of $309 / \mu 1$ compared to $121 / \mu 1$ in group A $(\mathrm{p}=0.23)$ and $94 / \mu 1$ in group $\mathrm{C}(\mathrm{p}<0.02)$. NK-cell recovery seemed somewhat more rapid in group $B(p<0.01)$ at 3 months only. Conclusions: These T-cell depletion regimens delay recovery of naïve T-helper cells. The use of Alemtuzumab plus CsA leads to a slow recovery of CD8+ T-cells also. After transplantation of highly purified peripheral blood CD34+ cells, B-cell recovery is exceptionally fast and durable.

\section{P523}

\section{Outcome of Allogeneic Hematopoetic Stem Cell Transplantation (HSCT) for Patients with Primary or Secondary Resistance to Imatinib in Chronic Myeloid Leukemia (CML)}

Koldehoff M., Elmaagacli A.H., Steckel N.K., Hlinka M.,
Ditschkowski M., Trenschel R., Peceny R., Beelen D.W.
Department of Bone Marrow Transplantation, University Hospita Department of Bone

Purpose: CML is a myeloproliferative disease that originates in an abnormal pluripotent bone marrow stem cell and is consistently associated with the Philadelphia $(\mathrm{Ph})$ chromosome and/or the BCR/ABL fusion gene. Imatinib, an orally administered 2-phenylaminopyrimidine derivative that inhibits BCR/ABL tyrosine kinase activity, has shown great promise in the treatment of CML. Resistance to imatinib therapy is clearly a barrier to successful treatment of CML patients (pts). Resistance to imatinib is multifaceted and is not easily defined. The only potenially curative therapy is an allogeneic HSCT. Methods and Results: Between December 2000 and Feburary 2004, 22 imatinib resistant pts (13 male, 9 female) with $\mathrm{Ph}$ positive CML underwent allogeneic HSCT. The median age at diagnosis was 36.5 (20-60) years and at transplant 39.9 (21-63) years. All pts received a prior induction chemotherapy $(n=10)$ or cytoreductive therapy $(n=12)$ followed by allogeneic HSCT. Conditioning therapy included myeloablative regimens (19 TBI/CY and 2 TBI/Fludarabine) in all pts. Stem cell source was 16 PBSC, 2 BM and 4 CD34 enriched PBSC. Five pts received stem cell graft from HLA-identical sibling donor and 17 pts from matched unrelated donor ( 9 mismatched). 
Transplants contained a median of 8.02 X10E6 CD34+ cells/kgBW. GvHD prophylaxis consisted $12 \mathrm{CSA} / \mathrm{MTX}, 4 \mathrm{CSA} / \mathrm{MTX} / \mathrm{ATG}$, one CSA only, and 4 ATG only. Twenty one pts engrafted for granulocytes (ANC $>0.5 / \mathrm{nl})$ at median of 16.8 days (9-26) and 16 pts for platelets at 20.7 days (13-33), respectively. One pt had a primary transplant failure with progressive disease and an other pt had a secondary transplant failure. The most non hematological side effect grade $>3+4$ was mucositis. Early complete donor chimerism ( $>95 \%)$ was observed in 20/22 pts. Acute GvHD of grade $\geq$ II occurred in eleven pts and chronic GvHD in seven pts (two limited and five extensive), respectivity. TRM was observed in 5 cases: MOF $(n=1)$, sepsis $(n=2)$, enterale GvHD $(n=1)$, and opportunistic infection $(n=1)$. Three pts died of relapse. 14 pts $(63,6 \%)$ are still alive at median survival time of 479 days. In conclusion, in our series of pts with imatinib resistant CML treated with myeloablative allogeneic HSCT using related or unrelated donor may be cure pts with this disease without severe TRM.

\section{Poster Session: Stem Cell Biology}

\section{P524}

\section{Resistance of Human Bone Marrow Mesenchymal Stem Cells (hMSC) for Cytotoxic Treatment Is Associated with Low Levels of Caspase Activation}

Mueller L.P., Mueller T., Luetzkendorf J., Reichelt K., Simon H., Schmoll H.-J.

Department of Medicine IV Hematology/Oncology and Center of Applied Medical Research (ZAMED), Martin-Luther-University Halle, Germany

Purpose: MSC participate in the bone marrow microenviroment which sustains hematopoiesis. Recent reports suggest, that autologous human MSC might be of interest for various therapeutic approaches. However the biology and ability of in vitro expansion of these cells still remains obscure. In the present study we investigated hMSC from healthy as well as affected human bone marrow regarding their sensitivity for cytotoxic treatment. Methods: MSC were isolated from BM aspirates of patients with proven malignant BM infiltration or with a history of chemotherapy as well as from healthy donors. Phenotypical analysis was performed by flow cytometry. Single cell cultures were analyzed for their in vitro osteogenic and adipogenic differentiation potential (cytochemistry, RT-PCR). Sensitivity against cisplatin, vincristin and etoposide was investigated by SRB cytotoxicity assays determining IC90 values. Caspase activation was analysed in cytosolic extracts by colorimetric assays using specific substrates. Presence of active caspase fragments was analysed by western blotting. Results: MSC with typical phenotype were isolated from all patients and showed mesodermal multipotent differentiation capacity in single cell derived cultures. IC90 values for vincristin, cisplatin and etoposide were comparable to those of established resistant tumor cell lines but considerably higher than in pluripotent sensitive embryonal carcinoma cells (ECC). Treatment for $24 \mathrm{~h}$ with IC90 cisplatin doses resulted in a high degree of morphologically apoptotic cells. However little activity of caspase 2 and 3 and almost no activity of caspase 8 and 9 - which are all typically activated by cisplatin - was observed. Western blot confirmed the lack of caspase 8 and 9 activation. Conclusions: Cultured hMSC show a resistance to cytotoxic treatment. However, this seems not to be the result of an in vitro artefact as we demonstrated previously that MSC are present in BM of patients after high dose chemotherapy. Thus, hMSC appear to have an intrinsically high apoptotic treshold. The reduced amount or entire lack of caspase activation after cisplatin treatment suggests that distinct apoptotic pathways are suppressed in hMSC. This not only offers an explanation for their persistence in BM after cytotoxic therapy but also gives new insights into the biology and degree of differentiation of these cells.
P525

\section{Analysis of Genexpression of Primitive Hematopoietic Progenitor Cells}

${ }^{*}$ Wagner W. ${ }^{1}$, Ansorge A. ${ }^{2}$, Saffrich R. ${ }^{1}$, Wirkner U. ${ }^{2}$, Eckstein V. ${ }^{1}$, Schwager C. ${ }^{2}$, Blake J. ${ }^{2}$, Miesala K. ${ }^{1}$, Wein F. ${ }^{1}$, Ansorge W. ${ }^{2}$, Ho A.D.

${ }^{1}$ Department of Medicine V, University of Heidelberg, Heidelberg, Germany, ${ }^{2}$ Biochemical Instrumentation Programme, European

Molecular Biology Laboratory, Heidelberg, Germany

Purpose: Stem cells are characterized by their dual ability to self-renew and to differentiate into multiple cell types. A genome wide gene expression analysis could shed light on the molecular mechanisms that regulate hematopoietic stem cells. We have provided evidence that divisional kinetics could be exploited to separate stem cells into two fractions: a slow dividing fraction (SDF) that is predominantly associated with primitive function and self-renewal, and a fast dividing fraction (FDF) that divides symmetrically and predominantly proceeds to differentiation. In continuation with this line of research we have analyzed the global gene expression profiles of these two populations. Methods: The CD34+/CD38- fraction and the CD34+/CD38+ fraction were isolated from human umbilical cord blood. We have then separated the $\mathrm{CD} 34+/ \mathrm{CD} 38$ - population into a slow dividing fraction (SDF) and a fast dividing fraction (FDF) as described in detail previously. Gene expression analysis was performed with a novel Human Transcriptome Microarray (Wirkner U., Ansorge W. et al., manuscript in preparation) that represents 51,145 UnigeneSet-RZPD3 cDNA clones. To obtain additional information on primitive versus committed progenitor cells, gene expression profile of CD34+/CD38- cells, which are associated with more primitive function was compared with that of the CD34+/CD38+ cells that are committed to differentiation. Flow cytometry and semi-quantitative RT-PCR confirmed the differential expression of several genes. Results: In the CD34+/CD38- fraction 96 sequences were found to be at least two-fold over-expressed and 119 were suppressed in comparison to CD34+/CD38+ cells. In contrast in the $\mathrm{SDF}$ versus the FDF a larger proportion of genes was differentially expressed (942 sequences up-regulated and 794 down-regulated). Among the genes showing the highest expression levels in the SDF were the following: CD133, erg, cyclin g2, MDR1, osteopontin, clqr1, ifi16, jak3, fzd6 and hoxa9, a pattern compatible with their primitive function and self-renewal capacity. In comparison to three other published microarray studies we identified several genes that were up-regulated in different fractions of murine and human HSC including: HoxA9, Jak3, fzd6, mdr1 and rbpms. Furthermore we observed more podia-formation in the SDF and CD133 is located on the tip of these podia. Time-laps analysis of the cells in co-cultivation with a stromal feederlayer showed that hematopoietic progenitor cells were often tightly attached to AFT024 cells. The impact of cell-cell contact on gene expression profiles of primitive HSC is concurrently analyzed. Conclusion: We have demonstrated that the quiescent population of $\mathrm{CD} 34+/ \mathrm{CD} 38$ - cells represents a highly enriched stem cell population on a molecular level. These results have provided the foundation for further analysis of genes that are responsible for self-renewal and will contribute to a detailed understanding of the asymmetric division of stem cells.

\section{P526}

\section{Acquisition of CD34 Correlates with Increased Hematopoietic and Self-Renewal Activity of CD34-CD133+ Cord Blood Cells}

\section{Götze K.S., Schiemann M., Marz S., Jacobs V.R., Debus G.,} Peschel C., Oostendorp R.A.

${ }^{1}$ Department of Medicine III, ${ }^{2}$ Department of Microbiology and Immunology, ${ }^{3}$ Department of Gynecology and Obstetrics, Technical University of Munich, Germany, ${ }^{4}$ Department of Gynecology and Obstetrics, NeuPerlach Hospital, Munich, Germany

CD34 is a sialomucin expressed on hematopoietic cells, endothelial cells, and muscle satellite cells. Within the hematopoietic system, CD34 expression has been associated with very immature progenitor cells as well as hematopoietic stem cells (HSC), and it is widely used to assess stem cell activity in clinical protocols. In the past, HSC activity was thought to be retained exclusively in the subset of cells expressing CD34. This view has been challenged by recent observations in mice, in which HSC activity was also found in the CD34negative cell fraction. These findings have since been reproduced using 
human marrow and cord blood cells. However, the exact relationship between CD34+ and CD34- stem cells remains unclear. We investigated the regulation of CD34 expression as dependent on cell division history. To follow cell division, human cord blood cells were labeled with the fluorescent dye CFSE. Lin-CD34-CD133+CFSE+ (CD34-) and CD34+ populations were almost indistinguishable in their ability to produce $\mathrm{CAFC}_{\text {week } 6}$ content. After 3 days of serum-free culture with stem cell factor, Flt3 ligand and thrombopoietin, almost all initially CD34- cells had acquired expression of CD34, including all undivided cells. In cultures initiated from CD34- cells, virtually all $\mathrm{CAFC}_{\text {week6 }}$ were produced from the divided CD34+ cells, indicating these cells had self-renewed. In contrast, similar cultures from initially CD34-positive cells demonstrated that hematopoietic activity associated with the undivided cell fraction. Analysis of mRNA expression showed that CD34- and CD34+ cells expressed almost equal levels of CD34, AC133, Flt1, Flk1, and Flt4, while CD34- cells expressed significantly lower levels of Tie1 and Tie2 than CD34+ cells. CD34 cells that did not express CD34 on their cell surface exhibited intracellular CD34, suggesting these cells were "primed" to express CD34. In conclusion, LinCD34-CD $133^{+}$cells acquire the expression of $\mathrm{CD} 34$, even in the absence of cell divisions. These CD34- cells self-renew more rapidly in vitro than cells initially expressing CD34, and self-renewal is preceded by acquisition of CD34 antigen.

\section{P527}

Expression and Regulation of the Cyclosporin A-sensitive Transcription Factor NFAT (Nuclear Factor of Activated T cells) in CD34+ Cells

Kiani A., Habermann I., Oelschlaegel U., Boxberger S., Feldmann S., Thiede C., Bornhäuser M., Ehninger G.

Department of Medicine I, Dresden University of Technology, Dresden, Germany

Transcription factors of the NFAT (Nuclear Factors of Activated T cells) family are established as one of the key elements of inducible gene expression in activated $\mathrm{T}$ cells and are considered to be the main molecular targets of the immunosuppressive drugs cyclosporin A and FK506. While regulation and function of NFAT proteins in lymphocytes has been extensively studied, little is known about their expression and potential biological relevance in myeloid cells. We have recently shown that NFATc2 (NFAT1) protein is expressed in $\mathrm{CD}_{3} 4^{+}$cells, but not in peripheral blood neutrophil granulocytes and monocytes. In the current study, we analyzed the expression of all five known NFAT family members on the mRNA level in $\mathrm{CD}_{3} 4^{+}$cells of cord blood and G-CSF-mobilized peripheral blood, in peripheral blood CD $34^{+}$cell subsets, and during the differentiation of $\mathrm{CD} 34^{+}$cells into neutrophil granulocytes. In $\mathrm{CD}_{3} 4^{+}$populations of both peripheral blood and cord blood NFATc3 was the NFAT family member found to be most prominently expressed by quantitative RT-PCR. Transcript levels for all NFAT family members were present at equal or higher levels in the immature $\mathrm{CD} 34^{+} \mathrm{CD} 38$ subset of peripheral blood $\mathrm{CD} 34^{+}$cells, as compared to $\mathrm{CD} 34^{+} \mathrm{CD} 38^{+}$or bulk $\mathrm{CD} 34^{+}$populations. Cytokine-induced in vitro differentiation of $\mathrm{CD} 34^{+}$cells into neutrophil granulocytes resulted in the rapid and almost complete suppression of NFATc2, but not NFATc3 transcripts. Dephosphorylation/ rephosphorylation as well as nuclear/ cytoplasmic translocation of NFAT in $\mathrm{CD}_{3} 4^{+}$cells followed the same calcineurin-dependent pattern as in T lymphocytes, suggesting that NFAT activation in these cells is equally sensitive to inhibition with cyclosporin A. Finally, in vitro proliferation of $\mathrm{CD} 34^{+}$cells cultured in the presence of FLT3-L, SCF, GM-CSF, IL-3 and G-CSF was profoundly inhibited by treatment with cyclosporin $\mathrm{A}$ in a dose-dependen manner; in contrast, their differentiation into neutrophil granulocytes remained unaffected. These results suggest a novel and unexpected role for members of the NFAT transcription factor family in the hematopoietic system.
P528

Homing and Long-Term Engraftment of Hematopoietic Stem Cells in Various In Vitro Niches - Expressionpattern of Adhesive Molecules and Ligands of the Notch-Pathway as Predictive Factor

Gottschling S. ${ }^{1}$, Eckstein ${ }^{1}{ }^{1}$, Jonas $A .{ }^{2}$, Krause U. ${ }^{1}$, Seckinger $A .{ }^{1}$, Saffrich R. ${ }^{1}$, Uhrig M. ${ }^{3}$, Wuchter $P^{1}{ }^{1}$, Ho A.D.

${ }^{1}$ University Hospital Heidelberg, Dept. of Internal Medicine $V$,

Heidelberg, Germany, ${ }^{2} \mathrm{EMBL}$, Heidelberg, Germany, ${ }^{3} \mathrm{ZMBH}$,

Heidelberg, Germany

Purpose: Several transplantation studies in animal and human models demonstrated preferential homing and long term engraftment of bone marrow-derived stem cells in hematopoietic environments and to a lesser extent in non-hematopoietic tissues like skin, gut, lung or brain. We have recently demonstrated the vital role of cell-cell contacts, especially between hematopoietic stem cells (HSC) and the stromal environment, not only for stem cell homing, but also on the long-term fate of HSC. In this study we have quantified and compared the homing and stem cell supporting properties of various in vitro microenvironments. Characterization of the environments for the expression of adhesive and cell-fate regulating molecules revealed a strong correlation between the expression pattern and their functional properties. Methods and Results: CD $34^{+} / 133^{+}$HSC were cocultured for up to $24 \mathrm{~h}$ with the murine stromal cell line AFT24, human mesenchymal stem cells (MSC), a murine neuronal (NFL) and a murine astrocyte feeder layer (AFL). Determination of adhesion kinetics revealed significant differences in the affinity of HSC towards the different feeder layers. A high and stable adhesion for at least $12 \mathrm{~h}$ occured only in coculture with AFT024 $(37.6 \pm 12.7 \%$ adherent cells) and MSC (31.7 6.1\% \pm adherent cells). Adhesion to nonhematopoietic environments was either low with $10.3 \pm 4.2 \%$ adherent cells for NFL or instable with initially $33.6 \pm 2.8 \%$ adherent cells and $15.3 \pm 3.5 \%$ adherent cells after $12 \mathrm{~h}$ for AFL. Nevertheless HSC adhered firmly ( $\geq 100$ $\mathrm{pN}$ ) to the various microenvironments as measured by an optical trap. To assess the support of the various feeder layers for repopulating HSC a LTCIC assay in limiting dilution was performed. Conservation of long-term culture initiating cells was $59.7 \%$ in coculture with AFT024 and $60.2 \%$ in coculture with MSC, whereas astrocytes and neurons did not support LTC-IC at all. Characterization of the feeder layers by means of immunophenotyping, western blotting and immunofluorescence microscopy for main adhesive ligands (CD29, CD54, fibronectin, heparan sulfate proteoglycan perlecan) and cell fate regulating molecules of the notch-pathway (jagged-1, delta) resulted in a distinct expression profile correlating highly with the different functional properties of AFT024, MSC, NFL and AFL. Conclusions: These data demonstrate superior homing and stem cell supporting properties of AFT024 and MSC compared with the neuronal and astrocyte feeder layer. Besides that they show that the different functional properties of the various in vitro environments correlate highly with the expression pattern of certain adhesive and cell fate regulating molecules.

\section{P529}

\section{Characterization of Cellular Contacts in Human Mesenchymal Stem Cells}

Wuchter P. ${ }^{1}$, Boda J. ${ }^{2}$, Grund $C .{ }^{2}$, Kuhn $C .{ }^{2}$, Krause U. ${ }^{1}$, Seckinger A. ${ }^{1}$, Gottschling S. ${ }^{1}$, Franke W.W. ${ }^{2}$, Ho A.D. ${ }^{1}$ ${ }^{1}$ Department of Medicine V, University of Heidelberg, Germany, ${ }^{2}$ Division of Cell Biology, German Cancer Research Center, Heidelberg, Germany

Purpose: Mesenchymal stem cells (MSC) are a population of adherent, fibroblast-like cells from human bone marrow, that are capable to differentiate into bone, cartilage and adipose tissues and are therefore of high interest for regenerative medicine. They can be grown to confluency on plastic surfaces and form multiple intercellular contacts. Thus far, neither the isolation and culturing methods of these cells have been standardized, nor have the intercellular junctional complexes been characterized. Methods: We have systematically analyzed the molecular composition of cellular contacts between human MSC obtained from bone marrow aspirates from healthy voluntary donors, using a panel of antibodies specific for various components of tight junctions, gap junctions, adherence junctions and desmosomes, by light and electron microscopy and by biochemical analysis, including immunoprecipitation and RT-PCR. Results: In these studies we have made two surprising observations of fundamental significance. The MSC are inter- 
connected by occasional gap junctions and two types of frequent adhering junctions: (i) Typical puncta adhaerentia (PA), often clustered or even fused, and (ii) a system of extended, slender, tentacle-like cell processes (processus adhaerentes) bearing one or several groups of PA, often in terminal or preterminal position. Some of these processus adhaerentes can be remarkably long (20 to $400 \mu \mathrm{m}$ ). The molecular composition of these adhering junctions is also unique, as they comprise the transmembrane glycoproteins cadherin-11, $\mathrm{N}$-cadherin and syndecan-1, together with the cytoplasmic plaque proteins $\alpha$ and $\beta$-catenin and $\mathrm{p}-120$. These junctions have not been described in human MSC before and bear similarity to those described by us in primary mesenchymal stem cells of day 7 to 8.5 mouse embryos (Franke et al., 1982 1983). Conclusions: Our data indicate that MSC communicate with each other through junctions and junctional complexes. We hypothesize that MSC can embark on alternative differentiation pathways with different junctional and cytoskeletal patterns. Characterization of and understanding the role of such intercellular contacts and their correlation to specific differentiation programs and stages are being elucidated.

\section{P530 \\ Impact of Stem Cell Factor (SCF) on the Engraftment and Differentiation Capacity of Human Hematopoietic Stem Cells Transplanted in NOD/SCID Mice}

Drewel D., Müller G., Pissler N., Andreesen R., Hennemann B. University Hospital Regensburg, Germany

Purpose: The transplantation into irradiated immunodeficient NOD/SCID mice is a valid assay to detect pluripotent human stem cells with the ability of multilineage hematopoietic reconstitution. In this study, we investigated the impact of SCF on the engraftment of lineage depleted (lin-) peripheral blood cells and cord blood-derived mononuclear cells, and analysed the organ distribution of the transplanted cord blood cells with or without the addition of SCF. Methods and Results: In two experiments, 16 NOD/SCID mice were injected with lin- peripheral blood cells \pm SCF. Six weeks later the bone marrow of the animals was analysed by flow cytometry and PCR. 8 out of 8 mice in the SCF group showed multilineage hematopoietic engraftment, while in the control group no human cells could be detected. Then, cord blood cells were injected \pm SCF into irradiated NOD/SCID mice. Mice that received SCF showed a 2.7 fold higher proportion of human CD45+71+ cells within the bone marrow six weeks after transplantation as compared to the control group. In addition, human cells were detected by immunohistochemistry in liver and kidneys, but not in neural tissue and testes of the transplanted animals. The percentage of human cells increased by the addition of SCF within the liver from $0.06 \%$ to $0.13 \%$ and within the kidneys from $0.02 \%$ to $0.26 \%$. Conclusions: Our data indicate a profound increase in the engraftment capacity of the transplanted cells. The organ specific differentiation of the human cells detected in liver and kidneys of the recipients has to be determined.

\section{P531}

\section{Mesenchymal Stem Cells of Patients with Chronic Myeloid Leukemia do Not Carry the Philadelphia Translocation}

Gentilini C., Al-Ali K.H., Reinhardt A., Bartsch K., Lange T., Niederwieser $D$.

Department of Internal Medicine II, University of Leipzig, Germany

Purpose: Mesenchymal stem cells (MSC) play a crucial role in the regeneration of mesenchymal tissues and in the microenvironment of bone marrow cells. The role of MSC in hematological malignancies, however, remains to be further elucidated. Moreover little is known about the influence of MSC in the development and maintenance of the malignant clone in chronic myeloid leukemia (CML) patients. This disease is characterized by a reciprocal translocation between chromosomes 9 and 24, which gives rise to the BCRABL fusion product, called Philadelphia $(\mathrm{Ph})$ chromosome. Early works showed that hepatocytes precursors, found in the liver of CML patients can be $\mathrm{Ph}$ positive. Our intent was to elucidate if MSC isolated from patients with CML in different stages of the disease carry the $\mathrm{Ph}$ translocation. Results: MSC were isolated from bone marrow aspirates of 11 patients with CML at different stages of disease. Five patients were analyzed at diagnosis, two after allogenic stem cell transplantation, three on treatment with the tyrosine kinase inhibitor imatinib and one on treatment with interferon alpha in combination with hydroxyurea. Cells were isolated by density gradient methods, resus- pended in RPMI1640 medium containing 10\% fetal bovine serum and plated in culture flasks to adhere. After 4-5 weeks of culture the expression of several surface markers were detected by fluorescence activated cell sorter (FACS). The presence of the Ph chromosome was assessed by fluorescence in situ hybridization (FISH) and polymerase chain reaction (PCR). Results were then compared with those obtained by analyzing the whole bone marrow. Isolated cells grew to confluence after a few weeks of culture, appearing as an adherent, spindle shaped cell layer. MSC were defined by expression of the typical surface markers $\mathrm{SH} 2$ and $\mathrm{SH} 2$, as well as by absence of CD34, CD45 and CD14. MSC were then analyzed by FISH using probes for BCR-ABL. We could not detect the $\mathrm{Ph}$ translocation in any of the analyzed patients, even if it was present in the remnant bone marrow cells. Moreover, results did not change, if expression of BCR-ABL was measured by high sensitivity RTPCR. Conclusions: MSC of patients with CML are Philadelphia negative irrespective of the stage of disease and the treatment given, suggesting that these cells are not involved in the development of the malignancy.

P532

Preclinical Production of Human Lineage-Negative Cells from Nonmobilized Blood for Regenerative Stem Cell Therapy

Strunk D., Rohde E., Lanzer G., Linkesch W.

Dept. of Internal Medicine, Div. of Hematology \& Dept. of Blood

Group Serology \& Transfusion Medicine, Medical University, Graz, Austria

Purpose: Regenerative stem cell therapy (SCT) is currently tested in clinical trials based on the hypothesis that adult SC can repair ischemic and metabolic organ injury. The ideal type and source of cells for regenerative SCT are not yet defined. Lineage (Lin)-depletion is an experimental procedure capable to enrich all recently recognized SC types with regenerative potency. This study was performed to define a practicable MoAb cocktail for Lin-depletion and to test whether clinical scale Lin-depletion is possible. Methods: MoAbs (CD2/14/15/19/41/56/ GlycophorinA) were selected to specifically mark seven mature hematopoietic lineages. Lin7-negative $\left(\operatorname{Lin} 7^{\mathrm{NEG}}\right.$ ) cells were analyzed in peripheral blood ( $P B, n=9)$, mobilized PB (MPB, $n=5)$, umbilica cord blood (UCB, $n=5)$ and bone marrow aspirates $(B M, n=4)$ by flow cytometry. The hematopoietic, mesenchymal and endothelial differentiation potential of sorted Lin $7^{\mathrm{NEG}}$ cells was evaluated under standardized in vitro culture conditions. Preclinical Lin-depletion was tested with leukapheresis products from PB following good manufacturing practice (GMP) principles. Results: Lin $7^{\mathrm{NEG}}$ cells comprised $0.23 \pm 0.04 \%, 0.27 \pm 0.03 \%, \quad 0.53 \pm 0.07 \%$ and $0.49 \pm 0.03 \%$ of PB, MPB, UCB and BM, respectively. Basophils, CD $34^{+}$and dendritic cells constituted the major Lin $7^{\mathrm{NEG}}$ subpopulations $(84 \pm 2 \%, 90 \pm 3 \%$, $40 \pm 3 \%, 80 \pm 3 \%$, in PB, MPB, UCB, BM). Minor populations included CD7 and CD45 cells. In vitro differentiation analysis revealed the propagation of endothelial and mesenchymal phenotypes from Lin $7^{\text {NEG }}$ SC products. Substractive analysis confirmed that hematopoitic colony formation was restricted to the CD34+ fraction of Lin $7^{\mathrm{NEG}}$ cells from nonmobilized $\mathrm{PB}$. Preclinical CD2/14/15/19/56 (Lin5)-depletion after automated RBC and platelet reduction resulted in an up to 20-fold enrichment of $\mathrm{CD}_{3} 4^{+}$and CD34\% Lin ${ }^{\mathrm{NEG}}$ cells. Conclusions: A seven MoAb cocktail is sufficient to label $>99 \%$ NC in PB, MPB, UCB and BM. Preclinical Lin-depletion can be performed under GMP conditions from nonmobilized PB aphereses. Extensive in vitro analysis are currently underway to determine the functional capacity of $\operatorname{Lin} 7^{\mathrm{NEG}} \mathrm{SC}$ products from nonmobilized PB.

P533

Functional Activity of Receptors of the Hypothalamic Peptides Corticotropin-Releasing Hormone (CRH) and Orexins A/B in Primary Human CD34+ Stem and Progenitor Cells

Bork S. ${ }^{1}$, Steidl U. ${ }^{1,2}$, Diaz Blanco E. ${ }^{1}$, Ludwig A. ${ }^{1}$, Gräf $T^{1}{ }^{1}$, Haas $R^{1}{ }^{1}$, Kronenwett $R .^{1}$

${ }^{1}$ Dept. of Hematology, Oncology and Clinical Immunology,

Duesseldorf, Germany, ${ }^{2}$ Harvard Institutes of Medicine, Boston, USA

Recently, we have shown that primary human CD34+ stem and progenitor cells express numerous neurobiological genes, among them $G$ proteincoupled receptors such as corticotropin-releasing hormone (CRH) 1 and 2 receptors, orexin/hypocretin 1 and 2 receptors and GABA B receptor. Most 
interestingly, we found that the expression of these receptors is restricted to immature CD34+ cells suggesting that they play a role in developmentally early hematopoietic stem and progenitor cells (Steidl et al., Blood 2004). Those findings prompted us to examine the functional activity of CRH 1 and 2 receptors as well as orexin 1 and 2 receptors and to elucidate their physiological role in human CD34+ stem and progenitor cells. Therefore, immunomagnetically enriched CD34+ cells from the peripheral blood (PB) of GCSF-mobilized healthy donors were incubated with specific agonists and antagonists of these receptors. Afterwards, intracellular concentration of the second messenger cyclic adenosine monophosphate (cAMP) was measured by a competitive enzyme immunoassay (EIA) and compared to untreated cells. We found a significant decrease of cAMP by $28 \%$ upon stimulation with $\mathrm{CRH}(1 \mu \mathrm{M})$, and by $35 \%$ and $46 \%$ in response to orexin $\mathrm{A}(1 \mu \mathrm{M})$ anc orexin $\mathrm{B}(0.1 \mu \mathrm{M})$ indicating that these receptors are functionally active in human $\mathrm{CD} 34+$ cells and coupled to inhibitory $\mathrm{G}$ proteins. In order to assess whether this affects growth or differentiation of hematopoietic progenitor cells we examined the clonogenic growth of human CD34+ cells using methylcellulose colony-forming assays. PB-CD34+ hematopoietic progenitors from healthy donors were seeded in semisolid methylcellulose growth medium at densities of $2 \times 10^{3}$ cells $/ \mathrm{mL}$ with or without orexin A or B Colony numbers (colony-forming units granulocyte/macrophage [CFU-GM] and burst-forming units erythrocyte [BFU-E]) were counted after two weeks. Incubation with $0.1 \mu \mathrm{M}$ orexin $\mathrm{B}$ or orexin $\mathrm{A}$ resulted in a significant inhibition of growth of CFU-GM by $33 \%$ and $18 \%$, and of BFU-E by $38 \%$ and $29 \%$. In conclusion, $\mathrm{CRH}$ and orexin receptors show functional activity in CD34+ stem and progenitor cells suggesting a molecular interrelation of neuronal and hematopoietic signaling mechanisms in humans.

\section{P534}

\section{Expression of a Human Endogenous Retrovirus, Herv-K in Hematopoietic Cells}

\section{Karlic H. ${ }^{1}$, Eder G. ${ }^{1}$, Fillitz M. ${ }^{2}$, Koskela S., Pittermann E. ${ }^{\text {, }}$ Pfeilstöcker M. \\ ${ }^{1}$ Ludwig Boltzmann Institute for Leukemia Research and Hematology,} ${ }^{2} 3$ rd Medical Department Hanusch Hospital, Vienna, Austria

Purpose: In contrast to other human endogenous retrovirus families which are highly defective, HERV-K proviruses possess all types of viral genes which are known in exogenous retroviruses. In malignancy (tumor cell lines, breast cancer, melanoma as well as leukemias and lymphomas) but also in many normal tissues, including placenta HERV-K mRNA expression has been detected. In hematopoietic stem cells, an interaction of HERV-K with elements from vectors which are used for gene therapy may lead to unforeseen consequences. Thus, the purpose of this study was to localize HERV-K mRNA expression in hematopoietic stem cells of different sources. Methods: Quantitative real time PCR (RTQPCR) and RNA- in situ hybridization (RISH) for HERV-K was applied for detecting HERV-K expression in cytospins and smears of blood and bone marrow samples of patients with lymphomas (9 blood samples and 3 lymph nodes), myeloproliferative diseases, MPD (16), myelodysplastic syndromes MDS (10) and acute leukemias AL. In addition, 8 blood samples and 2 bone marrow samples from healthy donors as well as 9 cord blood samples and 20 apheresates from lymphoma patients containing PBSC were analyzed. From cord blood, CD34 positive cells were enriched using the DYNAL system. Analysis of the same HERV-K gene (pol) in genomic DNA from above mentioned sources served as control. Results: Relative mRNA levels of HERV-K in apheresates as well as in blood and bone marrow samples of patients with MDS, MPD and AML from subtypes with less than $1 \%$ CD34 positive cells were in the range of less than $2 \%$ in relation to housekeeping genes. However, in bone marrow samples of two AML and a CML blast crisis (all of them with more than 50\% CD34+ cells) as well as in CD34+ cells from cord blood (but not from apheresates or normal bone marrow) as well as in three lymph nodes of patients with B-cell lymphomas, an overexpression of HERV-K was detected and confirmed by RISH. Interestingly, an enhanced expression of HERV-K was detected in cord blood derived apoptotic CD34+ cells, whereas the relative amount of HERV in genomic DNA did not change in samples from different sources. Conclusions: Our data suggest that retroviral genes may be activated in CD34+ cells from bone marrow of leukemia patients and in lymph nodes of lymphomas. In cord blood, it appears to be associated with apoptosis. This may support a critical stance regarding the use of cord blood derived stem cells as an alternative to allogenous bone marrow transplantation. Similar amounts of HERV-K in different samples of genomic DNA of the same cells and the absence of HERV-K activation in normal bone marrow and PBSC indicates that activation of HERV-K is the result of regulation of gene expression rather than amplification.

Supported by Jubiläumsfonds der Österreichischen Nationalbank

\section{P535 \\ Different Behaviour of Magnetic Nanoparticles for Labeling of Mesenchymal and Haematopoetic Stem Cells}

\section{Mailänder V. ${ }^{1}$, Kern M. ${ }^{2}$, Ermisch L. ${ }^{1}$, Holzapfel ${ }^{2}{ }^{2}$, Landfester $K{ }^{2}$,} Wiesneth M. ${ }^{1}$, Schrezenmeier $H$.

${ }^{1}$ Department of Transfusion Medicine, Institute for Clinical Transfusion

Medicine and Immunogenetics, University of Ulm, Germany,

${ }^{2}$ Department of Organic Chemistry III, University of Ulm, Germany

Purpose: Various types of stem cells have attracted a lot of interest as it has been shown that they can repair organ tissues and functions in different rodent models. Migration and homing of these cells has become an important clinical aspect. One possibility of cell tracing is the use of magnetic particles which can be detected by magnetic resonance tomography (MRT). Methods: Two commercially available magnetic nanoparticles (Feridex ${ }^{\circledR}$ and Resovist ${ }^{\circledR}$ ) were used for labeling mesenchymal stem cells (MSCs), HeLa and CD34+ peripheral stem cells. The cells were incubated with the nanoparticles $(25 \mu \mathrm{g}$ $\mathrm{Fe} / \mathrm{ml}$ ) with or without $1,25 \mu \mathrm{g}$ poly-L-lysine (PLL)/ml for $24 \mathrm{~h}$, then washed and histochemically stained by the Prussian blue reaction. Cells containing iron particles were further digested in a mixture of perchloric acid and nitric acid. Iron uptake was determined by a ferozine based method. Detection of cell suspensions in 5\% gelatin was performed in a standard MRT scanner. Results: MSCs coincubated with PLL and stained by Prussian blue showed an uptake of Feride $x^{\circledR}$ and Resovist ${ }^{\circledR}$. Quantitative measurement revealed 1520 pg Fe/cell. If Resovist ${ }^{\circledR}$ or Feridex ${ }^{\circledR}$ was used alone in MSCs, only with Resovist $^{\circledR}$ a detectable amount of iron was noted (2 pg Fe/cell). By increasing the concentration of Resovist ${ }^{\circledR}$ to $250 \mu \mathrm{g} \mathrm{Fe} / \mathrm{ml}$ MSCs were labeled with 10$12 \mathrm{pg} \mathrm{Fe} / \mathrm{cell}$. Electron microscopy showed that the iron particles were within cellular compartments. Similar results were obtained with HeLa cells. Uptake of magnetic nanoparticles in CD34+ cells was not detected by Prussian blue staining whereas other groups reported values of around $2 \mathrm{pg} \mathrm{Fe} / \mathrm{cell}$. For a reduction of the T2 signal to $50 \%$ to detect the labeled cells in MRT 250.000 cells/ml containing 10-20 pg Fe/cell were needed. Conclusions: In combination with a transfecting agent (PLL) Feridex ${ }^{\circledR}$ and Resovist ${ }^{\circledR}$ can be used to label cells for detection by MRT. Without PLL only Resovist ${ }^{\circledR}$ shows a significant uptake of iron particles in MSCs. Since the type of dextran (Feridex ${ }^{\circledR}$. dextran versus Resovist ${ }^{\oplus}$ : carboxydextran) is the most striking difference between these two magnetic nanoparticles we synthesized nanoparticles with different amounts of carboxylic side groups. Results are preliminary. Labeling of MSCs by magnetic particles seems feasible for clinical studies but is not yet sufficient in CD34+ stem cells.

\section{P536 \\ Fibrillar Collagen1 as Matrix for the ex Vivo Expansion of Haematopoietic Stems Cells}

\section{Steudel C. ${ }^{1}$, Oswald J. ${ }^{1,2}$, Jørgensen $B .{ }^{2}$, Salchert $K^{2}{ }^{2}$, Thiede $C^{1}{ }^{1}$, Ehninger G. ${ }^{1}$, Werner $\mathrm{C}^{1}{ }^{1}$, Bornhäuser M. ${ }^{1}$}

${ }^{1}$ Technical University Dresden, Medical Clinic I, Dresden, Germany, ${ }^{2}$ Institut of Polymer Research, Dresden, Dep. Biomaterials, Dresden, Germany

Purpose: The bone marrow is the natural environment of haematopoietic stem cells where they reside in close contact to stromal cells and extracellular matrix molecules. To mimic the native neighbourhood without additional feeder cells we used fibrillar collagen 1 as a matrix for ex vivo expansion of haematopoietic stem cells and analysed the effects on the cultured cells. Methods: Haematopoietic stem cells were enriched from umbilical cord blood by positive selection with magnetic beads coated with anti-CD34 antibodies. After one week of cultivation in the presence of reconstituted collagen 1 fibrils proliferation and surface markers were assessed and colony forming units assays were carried out. Additionally whole genome expression analysis was performed to identify differentially expressed genes and describe the impact of fibrillar collagen 1 on RNA level. For five selected genes chip data were verified by using real time PCR based on Taqman technology. Results: Presence of collagen1 in the culture medium slightly reduced the expansion of $\mathrm{CD} 34+$ cells but at the same time increased the number of colony forming units. Gene expression data indicated that several 
genes including inflammation related cytokines (i.e. IL1 and IL8) and cellcycle related proteins (i.e. BTG2, DUSP2) are up regulated in comparison to cultures without the fibrils. Conclusions: Contact with collagen seems to increase the potential of CD34+ cells to form colonies without increasing the number of cells. This could be correlated with the enhanced expression of cytokines and proteins with antiproliferative characteristics on RNA level. All together the results suggest that contact with collagen 1 result in a delay in cell cycle progression and reduced further differentiation into more committed cells.

\section{P537}

Short Term Repopulating Cells Obtained from Cultures of Human Cord Blood CD34+ Cells Retain Multi-Lineage Activity with a Low Output of Megakaryocytic and Erythroid Cells in Vivo

\author{
Fischer M. ${ }^{1}$, Schmidt M. ${ }^{1}$, Klingenberg S. ${ }^{1}$, Eaves $C .{ }^{2}$, \\ von Kalle C. ${ }^{3}$, Glimm $H^{1,4}$ \\ ${ }^{1}$ Dept. Of Internal Medicine 1, University, Freiburg, Germany, \\ ${ }^{2}$ Terry Fox Laboratory, BC Cancer Agency, Vancouver, Canada, \\ ${ }^{3}$ Exp. Hematol. Res. Foundation, $\mathrm{CCH}$, Cincinnati, USA, \\ ${ }^{4} \mathrm{IMMZ}$, University, Freiburg, Germany
}

Purpose: The clinical use of human hematopoietic cord blood (CB) transplants is hampered by a frequently associated delayed platelet recovery. We have previously shown that two types of human short term repopulating cells (STRC) can be enumerated by their ability to selectively engraft NOD/SCID$\beta 2 \mathrm{~m}-/-$ mice but not NOD/SCID mice and that these STRC may play an important role in the early neutrophil recovery seen in patients transplanted with cultured mobilized peripheral blood cells. Previous studies also showed that the delayed platelet reconstitution by $\mathrm{CB}$ transplants was likely due both to a low number of STRC in CB transplants and a reduced megakaryocytic and erythroid differentiation of individual CB STRC as compared to STRC in other types of transplants. Methods: To determine whether the numbers and in vivo differentiation activity of CB STRC could be modified in vitro, we have now analyzed the STRC present in suspensions of CD34+ CB cells before and after being cultured for up to 21 days in serum-free medium supplemented with EPO, flt-3 ligand, TPO, SCF, IL-6, IL-11, and IL-3. Results: Under these conditions, the number of CD41+ cells increased 40fold at day 14, total colony-forming cells increased 30-fold at day 8 and megakaryocytic colony-forming cells increased 14 -fold at day 8 . In contrast, serial bone marrow aspirates performed on a total of 172 NOD/SCID- $\beta 2 \mathrm{~m}-/$ mice transplanted with cells from the same experiments showed an initial 2 to 10 -fold decrease of STRC activity after 4 days in culture, followed by recovery of STRC-M and STRC-ML numbers to $25 \%$ and $80 \%$ of input values, respectively, by day 8 . Analysis of the size and lineage content of the STRC-M and STRC-ML clones generated in the mice showed no differences between the initial and culture-derived STRC. There was also no evidence of STRC that had become restricted to a particular myeloid lineage. Conclusions: Our findings demonstrate that the generation of STRC in cultures of CB CD34+ cells under conditions that support erythroid or megakaryocytic differentiation does not modify either their proliferative or their differentiation activity in vivo. This, in turn, suggests that such differences are developmentally regulated by mechanisms that cannot be readily activated by exposure to these conditions. The striking differences in the kinetics of in vitro defined progenitor populations and STRC also provide evidence that these assays detect distinct populations.

\section{P538}

\section{Microanatomy of Peripheral Collected Hemopoietic Stem} Cells: Mitochondria and C-Kit

Körper S., Wiesneth M., Schrezenmeier $H$.

Institute for Clinical Transfusionmedicine and Immunogenets and University Hospital Ulm, Germany

Purpose: Binding of stem cell factor (SCF) to c-kit can initiate intracellular $\mathrm{Ca} 2+$ release. Mitochondria are cellular $\mathrm{Ca} 2+$ buffer and play an important role in regulation of survival / apoptosis of haemopoietic cells. In excitable cells it has been shown that mitochondria are located on sides of $\mathrm{Ca} 2+$ release. In order to get more insight into mitochondrial location and activity and effects of progenitor cell mobilization in hemopoietic progenitor cells, we investigated the mitochondrial network of peripheral collected CD34+ hemo- poietic stem cells (HSC) and the distribution of c-kit on the cell surface of these cells. Methods: Laser scanning confocal microscopy in combination with different fluorescent probes was used to generate images from HSCs Mitochondria were stained with mitotracker green. CD117 (c-kit) was imaged with a PE marked monoclonal antibody. Image stacks were generated for individual HSCs. Independent mitochondrial or c-kit clusters were identified, the maxiumum size in the xy-direction (horizontal) and in the z-direction (vertical) was analysed. Results: 2 to 3 independent mitochondrial networks can be detected in HSCs. Normally there is a big network with a maximum extension in the xy-direction of $5.2 \pm 1.5 \mu \mathrm{m}$ and $7.5 \pm 2.0 \mu \mathrm{m}$ in the $\mathrm{z}$-direction. The smaller networks might contain only one mitochondrium and are $1.6 \pm 0.8 \mu \mathrm{m}$ (xy-direction) and $3.3 \pm 1.4 \mu \mathrm{m}$ (z-direction) in size. CD-117 is expressed in clusters on HSCs, an average of $4.2 \pm 2.4$ such c-kit clusters can be detected per cell and are not associated with the mitochondrial networks. The picture shows an (xy-direction) image of 3 mitochondrial networks of a HSC, the circle marks the location of c-kit

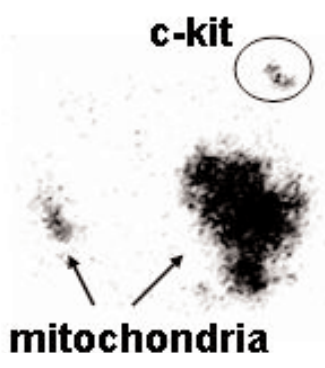

Conclusions: C-kit is expressed in clusters in HSCs and shows no colocalization with the mitochondrial networks of HSCs.

\section{P539 \\ Epithelial Chimeric Status of the Oral Mucosa after Human Hematopoietic Stem Cell Transplantation (HSCT)}

\section{Spyridonidis A., Zeiser R., Metaxas Y., Waterhouse M., Faber P.,} Finke $J$.

Dept. of Hematology / Oncology, University Medical Center Freiburg, Germany

Purpose: We recently reported that chimerism of the colon epithelium is a real phenomenon occurring constantly after human hematopoietic stem cell transplantation (HSCT). Moreover, we described the pitfalls of identification of epithelial chimerism and suggested stringent and unequivocal detection systems, like the use of combined stains and the use of laser scanning confocal microscopy. The purpose of the present study was to examine epithelial chimerism in the oral mucosa. Methods: Buccal scrapings obtained from 10 female patients (pts) who underwent a sex-mismatched allogeneic HSCT were used to prepare cytospins of buccal cells. The examination was performed 56 days to 1226 days after HSCT when the pts visited the BMT ambulance for regular examination. At this time point no patient had signs of mucositis or oral graft versus host disease (GvHD). Cytospin preparations were examined with a combination of fluorescence in situ hybridization (FISH) for the Y-chromosome, fluorescent stain for the epithelial specific marker cytokeratin (CK), CD45, and Drag cell nuclei stain; with APAAP immunocytochemistry using either CD45, CD15 or CD3 specific antibodies, and with hematoxylin/eosin (H/E) stain. Evaluation of epithelial chimerism was performed with laser scanning confocal microscopy from examiners who were not aware about the past medical history of the patients regarding mucositis or oral GvHD. Pts who received sex matched BM transplants were used as controls and showed hybridization specificity and $>95 \%$ FISH-Y detection sensitivity. Results: H/E stain of the cytospins revealed the presence of cells with an epithelial morphology. No CD15 positive cells were ever detected indicating the absence of macrophages having down-regulated CD45. CD45 or CD3 positive cells were found in 3 pts with a frequency of less than $1 \%$. In the FISH-Y combined stains we detected $\mathrm{Y}+/ \mathrm{CK}+/ \mathrm{CD} 45$ cells in $7 / 10$ pts $(70 \%)$ and with a median of $8.3 \mathrm{Y}+/ \mathrm{CK}+$ cells $/ 200$ total cells analysed (range 1-37). We retrospectively reviewed the transplantation documents of every pt and found a significant correlation ( $\mathrm{p}$-value $=0.0163$ ) between the severity of mucositis in the early post-transplant period (absent 3 ts, mild mucositis 3 pts, moderate 4 pts and severe 4 pts) and the degree of epithelial chimerism found at later time points $(d+56$ to $d+1226)$ where no signs of mucositis were present. Conclusions: Further clarification in the possible clinical implications of the epithelial chimerism could be given only 
by material examination of a great number of transplanted patients. Ora scrapings are easily and non-invasive obtained from pts and can be evaluated for degree of epithelial chimerism with the method described here. In order to further elucidate the mechanisms of this biological phenomenon we recently established PCR-based methods of laser-microdissected nuclei. Currently, we are evaluating microsatellite STR markers, DNA rearrangement, SNPs or germline DNA configuration in nuclei isolated from single or 5-10 pooled $\mathrm{Y}+/ \mathrm{CK}+/ \mathrm{CD} 45-$ cells

\section{P540}

Different Types of Transplantable Human Cord Blood Cells Display Different Aldehyde Dehydrogenase Activities

Christ O., Hamilton M., Leung K., Smith C., Eaves C.

Terry Fox Laboratory, BC Cancer Agency, Vancouver, BC, Canada

Cells expressing aldehyde dehydrogenase (ALDH+ cells) can be isolated by FACS based on their ability to convert freely diffusible BODIPY-aminoacetaldehyde (BAAA) into an intracellularly-retained, negatively-charged fluorescent derivative (BODIPY aminoacetate, BAA). We now report that $0.9 \pm 0.1 \%$ of low-density cord blood (CB) cells are ALDH+ (11 exp'ts) and removal of mature cells expressing lineage markers increases this proportion $\sim 10$-fold. $81+1 \%$ of ALDH+ CB cells with low light side scattering characteristics (SSClow) were found to be CD34+ and most of these cells did no express CD38. ALDH+ SSClow CB cells are also highly enriched in their content of primitive progenitors defined functionally; i.e., CFCs and LTC-ICs (enriched 80 -fold and 220-fold, respectively). Transplantable cells able to regenerate human lympho-myelopoiesis for at least 16 weeks after intravenous injection into sublethally irradiated immunodeficient NOD/SCID mice were found exclusively in the ALDH+ SSClow fraction of CB cells at a frequency of $1 / 4,700$. Additional removal from the ALDH+ SSClow population of cells expressing markers not characteristic of transplantable human hematopoietic cells allowed these to be purified a further 10-fold. In contrast, $\mathrm{CB}$ cells that produce a transient output of myeloid progeny for 3-6 weeks in the same mice appeared to be equally distributed between the ALDH+ and ALDH- subsets of SSClow CB cells. These results provide further support for the view that human $\mathrm{CB}$ contains biologically distinct and prospectively separable subpopulations of cells that regenerate mature blood cells in vivo at different rates and for different periods of time, and that these cell types can be distinguished by measureable differences in their ALDH activity.

\section{P541}

\section{Fetal Calf Serum (FCS) Free Isolation and Expansion of Human Mesenchymal Stem Cells (MSC) for Clinical Use (Osteogenic Differentiation)}

\section{Stute N. ${ }^{1}$, Holtz K. ${ }^{1}$, Bubenheim M., Lange C. ${ }^{1}$, Blake F. ${ }^{3}$, Zander A.R. ${ }^{1}$}

${ }^{1}$ Bone Marrow Transplant Center, ${ }^{2}$ Biostatistics, ${ }^{3}$ Oral and Maxillofacial Surgery, University Hospital Hamburg-Eppendorf, Germany

Introduction: Mesenchymal stem cells (MSC) according to Caplan or Prockop et al. are isolated and expanded in FCS-containing media and are multipotent. MSC have already been used in the clinic for children with osteogenesis imperfecta or metabolic diseases, and immunomodulation in the prevention and treatment of GvHD in allogeneic stem cell transplantation. Further applications in cell therapy are being developed in animal models and may enter the clinic soon. One major hurdle are problems related to FCS, such as viral infections, prions (BSE) and allergic reactions, which cannot be excluded by several washings after expansion. Therefore, considerable efforts are directed towards the development of FCS-free media for clinical use. In particular, isolation of MSC from bone marrow has so far proven elusive without the use of FCS. Methods: Nine donors gave $5 \mathrm{ml}$ of bone marrow aspirate each. Isolation of MSC was conducted according to Caplan et al., and expansion used a modified protocol with low-density seeding. Medium D consisted of DMEM low glucose with $10 \%$ of our selected FCS (gold standard), while media A, B and C were FCS-free. Isolation and expansion were performed on surface-treated 24-well plates and in flasks. Cells were counted under the microscope daily or every 3 days. Osteogenic differentiation was induced by a standard cocktail with $\beta$-glycerophosphate being added after 9 days. Adipogenic differentiation was likewise induced by a standard cocktail repeated twice. Differentiation was evaluated semiquantitatively with the use of the microscope and standardized scales. Results: Isolation of MSC-like cells was best in media $\mathrm{C}$ with $\mathrm{C}>\mathrm{D}>\mathrm{B}>\mathrm{A}$. Proliferation was exponential with $\mathrm{C}>\mathrm{D}>\mathrm{B}>\mathrm{A}$. Morphologically, MSC isolated and expanded in medium $\mathrm{C}$ were indistinguishable from those in medium D. Phenotypic markers of MSC grown in medium $\mathrm{C}$ and analyzed by flow cytometry were: CD34-, CD45-, MHC class I-, CD90+, CD105+, MHC class II+, similar to MSC isolated and grown in medium D. Moreover, MSC from medium C showed more osteogenic potential in the von Kossa assay than those from medium $\mathrm{D}$ in all cases: $\mathrm{C}+++, \mathrm{D}++, \mathrm{B}+, \mathrm{A} 0$. Cells retained their immaturity as shown by adipogenic differentiation: $\mathrm{D}+++, \mathrm{C}++, \mathrm{B}+, \mathrm{A} 0$. Conclusions: We were able to demonstrate that growth of MSC in a FCS-free medium is feasible without addition of FGF (fibroblast growth factor). Based on our data, we conclude that medium $\mathrm{C}$ is as good as our $10 \%$ FCS-containing medium D with regard to both isolation and expansion of MSC, and in particular for osteogenic differentiation, while media A and B are inferior. Seventy million MSC can be grown within 4 weeks. Details of media A, B and C will be provided at the meeting.

\section{Poster Session: Acute Lymphoblastic Leukemia}

\section{P542}

\section{Combination of Imatinib mesylate $\left(\right.$ Glivec $\left.^{\circledR}\right)$ and} Interferon- $\alpha$ (IFN- $\alpha$ ) maintains complete hematologic remission in minimal residual disease positive (MRD+) Philadelphia-positive acute lymphoblastic leukemia (Ph+ALL)

Wassmann B., Pfeifer H., Brück P., Bug G., Binckebanck A., Käbisch A., Lübbert M., Claus B., Giagounidis A., Planker M., Caspari R., Gschaidmeier H., Hoelzer D., Ottmann O.G. For the GMALL Study Group

Background: Remission duration in patients with advanced $\mathrm{Ph}+\mathrm{ALL}$ treated with single agent Imatinib is brief due to rapidly acquired resistance. IFN- $\alpha$ may enhance the antileukemic activity of Imatinib. We evaluated the impact of combined treatment with Imatinib and low-dose IFN- $\alpha$ in patients with $\mathrm{Ph}+\mathrm{ALL}$ who either were in complete hematologic but not molecular remission (CHR) during Imatinib monotherapy or were refractory to single-agent Imatinib. Patients: Median age of the 15 pts. in CHR who were MRD+ with Imatinib alone (group I) was 64 years. Imatinib was given for relapsed or refractory $\mathrm{Ph}+\mathrm{ALL}(\mathrm{n}=2)$, persisting bcr/abl positivity after chemotherapy $(n=4), 1^{\text {st }}$ line therapy in elderly patients $(n=5)$ or nonhematologic toxicity precluding further chemotherapy $(\mathrm{n}=2)$. Four pts. had previously undergone allogeneic $(n=3)$ or autologous $(n=1)$ SCT. Subcutaneous IFN- $\alpha$ was added a median of 4.0 months after starting Imatinib, with dose esacalation to a target dose of $3 \mathrm{MU} 3 \mathrm{x} /$ week if tolerated. Six pts. with Ph+ALL were enrolled because of refractoriness to single-agent Imatinib (group II) administered for relapse $(n=5)$ or bcr/abl positivity after a $2^{\text {nd }}$ alloSCT $(n=1)$. All pts. in this group had received previous SCT (allogeneic $n=5$, autologous $n=1$ ). Results: Nine of 15 pts. (60\%) enrolled in the setting of MRD positivity (Group I) remain in CHR a median of 10 months (range 1.4-29.3+ mo.) after adding IFN- $\alpha$, two pts. converted to MRD negativity. Three pts. developed BM relapse within 2.9 mo. after adding IFN- $\alpha, 2$ pts. without prior CNS-irradiation developed isolated CNS-relapse during combination or shortly after its discontinuation, one pt. died in CHR from septicaemia. Estimated progression free survival (PFS) at $24 \mathrm{mo}$. is $58.4 \pm 14.4 \%$ and OS at 12 and $24 \mathrm{mo}$. is $68.9 \pm 13.1 \%$ and $51.7 \pm 17.9 \%$. In contrast, 5 of 6 pts. with Imatinib refractory disease (group II) progressed within 2.4 mo. (range 0.2-5.0 mo.) after IFN- $\alpha$ addition. One pt. was censored after $1.5 \mathrm{mo}$. at the time of alloSCT. Estimated PFS at 5.0 months and OS at 13.9 months are 0\%. Conclusion: Combination treatment with Imatinib and low-dose IFN- $\alpha$ sustains prolonged hematologic remissions in patients with $\mathrm{Ph}+\mathrm{ALL}$ if started in the setting of minimal residual disease. Conversely, additional IFN- $\alpha$ has limited or no benefit in $\mathrm{Ph}+\mathrm{ALL}$ patients who are refractory to single agent Imatinib at the hematologic rather than the molecular level. 
P543

\section{Treatment of Childhood Acute Lymphatic Leukemia in Mongolia according to the BFM95 Protocol}

\author{
Schumm M. ${ }^{1}$, Chimgee G. ${ }^{2}$, Bjambaa $B .^{2}$, Purevsuren $A .{ }^{2}$, \\ Najmaa $P .{ }^{2}$, Bruchelt G. ${ }^{1}$, Feuchtinger T. ${ }^{1}$, Niethammer D. ${ }^{1}$, \\ Choijamts $\mathrm{G}^{2}$ \\ ${ }^{1}$ University Childrens Hospital Tübingen, Germany, ${ }^{2}$ Maternal and \\ Child Medical Research Center of Mongolia, Ulaan Baatar, Mongolia
}

Purpose: Acute lymphoblastic leukaemia is the most common leukaemia in children in Mongolia. The BFM95 protocol was introduced in 2002 to improve survival rate which was poor in the past. Methods: 17 patient were diagnosed in 2003 at the only treatment center for childhood leukaemia at the Maternal and Child Medical Research Center of Mongolia in Ulaan Baatar. Median age of 6 male and 11 female patients was 9.5 years (2-17 years) Results Patients suffer from bleedings (6), fatigue (7), bone pain (4) and had elevated white blood counts of median 13 x 10e9/1 (range 2-270). Diagnosis was confirmed by typical lymphoblasts in bone marrow (median 62 , range 11-93 percent). and peripheral blood ( median 35, range $1-77$ percent). 13 of 17 patients responded to induction therapy, 7 patients finished block I, 5 patients block M,. 2 patients finished block II and one patient entered maintenance therapy. To this day, 7 patients are alive and well, 4 patients left therapy, 5 patients died of sepsis and other infections, 1 patient died of bleedings. Conclusions: These results are encouraging by showing that the BFM95 has been introduced successfully in Mongolia. Main problems arose from severe infections and from the abortion of the therapy by the patients or their parents. In the future efforts are necessary to improve the management of infections and to improve the patients compliance.

\section{P544}

\section{Aleukemic AML Presenting as Nonimmune Hemolytic Anemia with Progressive Hepatosplenomegaly}

\section{Classen CF, Debatin K.-M.}

University Childrens Hospital UIm, Germany

Purpose: Presentation of an uncommon case of aleukemic ALL. Case Report: Aleukemic myeloid leukemia in childhood often leads to diagnostic difficulties. Both in the pediatric and the adult age group, localized infiltrate of AML are most typically found in the gingiva or the skin. Involvement of liver and spleen, but without overt leukemic disease, is rare. Hemolytic anemia as paraneoplastic condition in leukemia has more often been described in ALL, however, occasionally it is also seen in AML. In most cases it is immune-mediated. Here we describe a 3 years, 3 months old boy presenting with severe nonimmune hemolytic anemia, followed by massive hepatosplenomegaly. Sequential bone marrow examinations first showed reactive erythroid hyperplasia, then a progressively rigid cell infiltration nearly unaccessible to cytologic examination, with no specific histochemical markers. At no time, blast cells were visible in the peripheral blood. Liver biopsy was impossible due to refractory thrombopenia. Hepatosplenomegaly progressed to monstrous organ sizes, leading to life-threatening impairmen of respiration and digestion within four weeks. Probatory treatment with high-dose steroids had no beneficial effect. Since finally a very limited number of atypical blasts - most likely resembling an AML FAB M0 phenotype - was identified in the bone marrow, AML therapy according to the AML-BFM-1998-Interim protocol was initiatiated. After a transitory systemic inflammatory reaction with lung edema requiring artificial ventilation, this led to rapid clinical improvement; liver and spleen size returned to normal within two weeks, and the patient achieved a good clinical condition, with no signs of hemolysis or bone marrow insufficiency, and with normally reconstituted bone marrow after 4 weeks. Conclusion: We conclude that aleukemic acute myeloid leukemia must be considered as differential diagnosis in cases of nonimmune hemolytic anemia with massive hepatosplenomegaly.
P545

Treatment of Patients with ALL and Burkitt's Lymphoma According to the "GMALL Protocol 05/93" at the Department of Hematology \& Oncology, Innsbruck. A Unicenter Experience

Lackinger G., Gunsilius E., Nachbaur D., Wolf D., Rumpold H., Erdel M., Ulmer H., Gastl G., Petzer A.L.

University Hospital Innsbruck, Austria

Purpose: We performed a retrospective analysis on 43 newly diagnosed ALL-patients (pts) and 8 pts with Burkitt's lymphoma treated at our Department during 1996 - 2002. Methods: The latest update was performed in December 2002 with a median follow-up of 20.5 months. Results: Median age was 38 years (range:16 to 63 yrs). All pts received potentially curative treatment following GMALL protocol $05 / 93$. 76.5\% achieved a complete remission (CR) after first induction therapy cycle, $12 \%$ had a PR and $4 \%$ did not respond to first induction chemotherapy cycle. The early death rate was $7.8 \%$. After the $1^{\text {st }}$ year of therapy (including induction, consolidation and reinduction) $86 \%$ achieved a CR. The remission duration, however, was short (median 6 months) with 17/51 patients (33\%) even relapsing during ongoing therapy (both, on consolidation/reinduction or maintenance). The 5-years-OS of the ALL-pts was 33\% (median OS: 20 months) and 62.5\% in the Burkitt's lymphoma pts (24 months median OS). The OS of the 8 ALL-pts with a proven bcr-abl fusion gene in their initial cytogenetics was $18 \%$ at 4 years in comparison to $39 \%$ at 7 years in pts not harbouring the bcr-abl fusion gene. According to different therapy-arms patients within the B-ALL-therapy group (including pts with Burkitt's lymphoma; $\mathrm{n}=9$ ) had the best OS of 55.6 $\%$ at 5 years followed by pts that were treated according to the standard risk (SR) group with an OS of $45.5 \%(\mathrm{n}=25)$. High risk pts (B-precursor-ALL with $\mathrm{WBC}>30000 / \mu 1$, pre-pre-B-ALL or bcr-abl pos.; $\mathrm{n}=10$ ) and the T-ALLpts $(n=7)$ had a similar OS of $18.4 \%$ and $17.9 \%$ at 3 years. $19 / 51$ patients (37\%; incl. 2 pts with Burkitt's lymphoma) received an alloSCT (9 siblings, 10 MUD) due to relapse/refractoriness of the disease ( $8 \mathrm{pts}$ ) or due to a high risk constellation (10 pts), one was treated with an alloSCT from a sibling at $\mathrm{SR}$. The 3-years-OS of the pts transplanted due to high risk was 33\%, those of pts transplanted due to relapse/refractoriness was $18 \%$. Main causes of deaths (60\%) for all pts were fatal infections, $22 \%$ experienced disease progression, bleeding complications occurred in $3.7 \%(n=1)$, toxic drug effects occurred in $7.4 \%$ and other/unknown causes of death were found in $7.4 \%$. Conclusions: Our data are comparable with published data. An initial high CR rate in ALL pts is followed by frequent relapses. Future therapy protocols should therefore focus on improving maintenance strategies rather than on the induction phase. If possible, an alloSCT should be offered to all patients with high-risk ALL.

\section{Poster Session: Acute Myeloid Leukemia}

\section{P546}

High BAALC Expression Predicts Adverse Outcome in Acute Myeloid Leukemia with Normal Cytogenetics: Results of The SHG'96 Study

\section{Baldus C.D. ${ }^{1}$, Thiede C. ${ }^{2}$, Bloomfield C.D. ${ }^{3}$, Thiel E. ${ }^{1}$ Ehninger G. ${ }^{2}$}

${ }^{1}$ Charité, Universitätsmedizin Berlin, Campus Benjamin Franklin, Medizinische Klinik III, Berlin, Germany, ${ }^{2}$ Universitätsklinikum Carl Gustav Carus, Technische Universität Dresden, Medizinische Klinik I, Dresden, Germany, ${ }^{3}$ The Ohio State University, Comprehensive Cancer Center, Columbus, Ohio, USA

Purpose: High mRNA expression levels of the human gene BAALC (Brain And Acute Leukemia, Cytoplasmic) have been shown to be an adverse risk factor in newly diagnosed acute myeloid leukemia (AML) patients (pts) (Blood 2003;102:1613). The first study included 86 de novo AML pts under the age of 60 with normal cytogenetics and a more favorable FLT3 mutations status, which were uniformly treated on the Cancer And Leukemia Group B protocol 9621. An independent confirmation of these data is indicated to validate and expand the initial results, so that $B A A L C$ expression can be exploited for risk adapted treatment stratification of intermediate risk AML pts Methods: Here we present the results of a Süddeutschen Hämoblastosegruppe (SHG) study including 315 adult AML pts with normal cytogenetics, age $<60$ years and uniformly treated on the SHG' 96 protocol. Median followup for all patients was 14 months (range 0-86 months) and for the patients 
alive 27 months. BAALC expression was measured in pretreatment periphera blood blasts by comparative real-time RT-PCR. Pts having BAALC expression values within the lower $75 \%$ were classified as low BAALC and pts having $B A A L C$ expression values within the upper $25 \%$ were classified as high $B A A L C$. Results: There was no difference in the frequency of FLT3 internal tandem duplications between the high and the low BAALC group. Pts with high $B A A L C$ expression levels had an inferior overall survival (OS, median: 32 months vs. 45 months, $\mathrm{P}=0.03$ ), event-free survival (EFS, median: 9 months vs. 14 months, $\mathrm{P}=0.0026$ ), and disease-free survival (DFS, median: 9 months vs. 34 months, $\mathrm{P}=0.025)$. A multivariate analysis is in process to confirm that $B A A L C$ is an independent prognostic factor. Conclusions: This independent and larger study strongly supports the relevance of high $B A A L C$ expression as an adverse prognostic risk factor in intermediate risk AML pts with normal cytogenetics.

\section{P547}

Proteomic Pathway Discovery of C/EBP $\alpha-p 30$ Target Proteins in Acute Myeloid Leukemia: The C/EBP $\alpha-p 30$ AML Mutant Interferes with nuclear mRNA Processing

Geletu M., Christopeit M., Pulikkan J.A., Peer Zada A.A., Hiddemann W., Behre G.

Department of Medicine III, University Hospital Grosshadern, Ludwig - Maximilians-University, Munich, Germany

Recently, it has been reported that mutations in the transcription facto $\mathrm{C} / \mathrm{EBP} \alpha$ occur in $10 \%$ of patients with acute myeloid leukemia (AML), which often leads to the expression of an N-terminal, truncated, $30 \mathrm{kDa}$ isoform. The mutant protein blocks the wild type C/EBP $\alpha$ DNA binding and transactivation of granulocyte target genes in dominant negative manner and fails to induce granulocytic differentiation. In the present study, we applied a global proteomics approach (2D gel electrophoresis and MALDI-TOF mass spectrometry) to identify the target proteins of $\mathrm{C} / \mathrm{EBP} \alpha-\mathrm{p} 30$. Furthermore, we demonstrate protein pathway discovery on a global level using the ingenuity protein pathway finder software. We used K562 cells stably transfected with a $\mathrm{C} / \mathrm{EBP} \alpha-\mathrm{p} 30$ expression plasmid under the control of a beta estradiol inducible promoter as model system. Our preliminary results suggest that $\mathrm{C} / \mathrm{EBP} \alpha-\mathrm{p} 30$ modulates the expression of many proteins including signa transduction regulators, nucleic receptors, metabolic enzymes, oncogenic proteins, heat shock proteins, proteins involved in oxidation and reduction, structural proteins and nuclear mRNA processing and export proteins such as hnRNPA1, A2 and JKTBP1delta6, activated RNA polymeraseII transcriptional co-activator p15(TCP4) and translation elongation factor EF-TU(p43). These data together with data from primary patients with $\mathrm{N}$-terminal mutation of $\mathrm{C} / \mathrm{EBP} \alpha$ suggest that $\mathrm{C} / \mathrm{EBP} \alpha-\mathrm{p} 30$ target proteins could be novel biomarkers for the treatment of leukemia. Furthermore, it shows protein networks in human granulopoesis and how the balance between proliferation and differentiation can be disrupted in AML.

\section{P548}

\section{C-RAF Mutations in Therapy-Related Acute Myeloid Leukemia}

Zebisch A., Staber P.B., Fischereder K., Bodner C., Hiden K. Linkesch W., Auner H.W., Windpassinger C., Höfler G., Sill H. Division of Hematology, Department of Internal Medicine, Institute of Medical Biology and Human Genetics, and Department of Pathology, University Hospital of Graz, Austria

Purpose: Constitutive activation of the RAS-RAF-MEK-ERK signaling cascade plays an important role in the pathogenesis of several malignancies including acute myeloid leukemia (AML). We were interested whether abnormalities of the RAF proto-oncogenes occur in AML and whether they constitute potential targets for small molecule inhibitors and antisense strategies. Methods: cDNA expression of A-, B-, and C.RAF was assessed by realtime quantitative (rtq) PCR in 102 patient samples of AML, three AML cell lines and normal CD34+ cells of seven individuals. The results were correlated with the phosphorylation status of ERK, as measured by immunoblo analysis. Furthermore, we screened for gene amplifications by rtq PCR and mutations by PCR and sequence analysis. In samples with RAF mutations we additionally searched for N-RAS point mutations and FLT3 internal tandem repeats. Finally, frequency of RAF germline mutations was determined in the general population by performing a denaturing HPLC of 200 healthy individ- uals. Results: Forty-five of $82(54,9 \%)$ AML cases showed ERK phosphorylation indicating constitutive activation of the RAS-RAF-MEK-ERK pathway. A statistically significant correlation among RAF isoform expressions could be shown indicating their common upstream regulation. However, there was no correlation between ERK phosphorylation and the expression levels of either A-, B- or C-RAF. Deletions of the B-RAF gene were observed in 20/99 (20\%) cases and were in concordance with cytogenetic results. Interestingly, in the samples of therapy-related AML one ARAF gene duplication and two novel C-RAF missense mutations were detected: $\mathrm{S} 427 \mathrm{G}$ in one and $\mathrm{I} 448 \mathrm{~V}$ in the other patient. Both mutations are located within the protein kinase domain and are associated with strong ERK activation, however, no RAS or FLT3 abnormalities were detected in these samples. Screening constitutional and primary tumor DNA revealed these mutations of germline origin with a frequency in the healthy population of $<1 / 400$. Conclusions: We have confirmed the high prevalence of a constitutively activated RAS-RAF-MEK-ERK pathway in AML and furthermore identified two novel C-RAF germline missense mutations in therapy-related AML. Since both C-RAF mutations are associated with constitutive activation of the pathway, they may represent a novel target for specific therapeutic interventions in patients with therapy-related AML.

\section{P549 \\ Identification of Genomic Imbalances in AML with Complex Karyotype using Matrix-Based Comparative Genomic Hybridization}

Rücker F.G. ${ }^{1}$, Schwänen C. ${ }^{1}$, Lipka D. ${ }^{3}$, Wessendorf S. ${ }^{1}$, Fröhling S. ${ }^{1}$, Kestler H. ${ }^{4}$, Lichter $P^{2}{ }^{2}$, Bentz M. ${ }^{1}$, Döhner K. ${ }^{1}$, Döhner $H^{1}{ }^{1}$

${ }^{1}$ Department of Internal Medicine III, University Hospital of Ulm, Germany, ${ }^{2}$ Division of Molecular Genetics, German Cancer Research Center, Heidelberg, Germany, ${ }^{3}$ Department of Internal Medicine III, University Hospital of Mainz, Germany, ${ }^{4}$ Division of Neuroinformatics, University of Ulm, Germany

Approximately 10 to $15 \%$ of acute myeloid leukemia (AML) exhibit complex karyotypes, i.e. three or more chromosome abnormalities without presence of a specific fusion transcript. Using chromosome banding analysis, the majority of such cases cannot be completely described due to the low resolution of this method. Comparative genomic hybridization to microarrays (matrix-CGH) is a novel technique that allows genome-wide screening for genomic imbalances at high resolution and thus may facilitate the identification of new regions harboring potential disease-related genes. We designed a microarray consisting of 2799 different human genomic DNA fragments cloned in bacterial artificial chromosome (BAC) or P1-derived artificial chromosome (PAC) vectors. A set of 1502 of these clones covers the whole human genome with a physical distance of approximately $2 \mathrm{Mb}$. The remaining 1297 clones either contiguously span genomic regions known to be frequently involved in hematologic malignancies (e.g. 1p, 2p, 3q, 7q, 9p, 11q, $12 q, 13 q, 17 p, 18 q)(n=610)$ or contain oncogenes or tumor suppressor genes $(\mathrm{n}=687)$. In a first series we studied 30 AML cases with complex karyotypes. Genomic losses were more frequent than gains. The most frequent aberration was deletion of chromosome $5 \mathrm{q}$ ( 26 of 30 cases), followed by genomic losses affecting chromosomes 7q (53,3\%), 17p (50\%), 20q (26,7\%), 12q $(23,3 \%)$, $16 \mathrm{q}$, and $18 \mathrm{q}(26,7 \% \mathrm{each})$. Genomic amplifications were identified in 12 cases. These amplifications were located in chromosomal bands 8q24, 9p21p24, 10p13-p15, 11q13-q23.3, 12p13, 13q12-q14, 20q11.1, 21q22, and $22 q 11-q 12$. Our preliminary data demonstrate the potential of matrix-CGH with regard to spatial resolution and sensitive detection of genomic imbalances. Analysis of a large series of AML cases with complex karyotype may lead to the identification of novel candidate regions and pathogenetically relevant genes.

\section{P550}

\section{Activating Mutations of the SHP-2 Protein Tyrosine Phosphatase in Adult Patients with Acute Myeloid Leukemia}

Thiede C., Steudel C., IIImer T., Schaich M., Ehninger G.

Medizinische Klinik 1, Universitätsklinikum Carl Gustav Carus, Dresden, Germany

Purpose: Acute myeloid leukaemia (AML) represents a heterogeneous group of early stem cell malignancies characterized by an uncontrolled expansion of malignant cells blocked at certain stages of myeloid differentiation. Several 
abnormalities have been described, including translocations involving genes of the $\mathrm{CBF}$ family, as well as activating mutations of signal transduction proteins, e.g. N-RAS, K-RAS or the Flt3- receptor tyrosine kinase (RTK). More recently, mutations in the SHP-2 protein, a tyrosine phosphatase involved in RTK signal transduction and regulation, have been described in patients with juvenile myelomonocytic leukaemia (JMML) as well as paediatric patients with AML. In order to investigate the prevalence and the prognostic relevance of this gene in adults, we analyzed the mutational status of SHP-2 in a cohort of patients with AML. Patients and Methods: DNA prepared from bone marrow or peripheral blood samples taken at diagnosis from 426 patients treated in the AML96 protocol of the SHG Dresden was analyzed. Screening for mutations in the mutational hot spot in exon 3 of the PTPN11 gene coding for SHP-2 was performed on a dHPLC system (Transgenomic). Samples with aberrant dHPLC chromatogram were sequenced. Results: In the 426 patients investigated so far, 13 PTPN11 mutations were found $(3.1 \%)$. All mutations consisted of single base pair exchanges in codons 60-76, most mutations occurred in codons 69 (3) and 72 (3). No association with age and other clinical parameters was found. Seven patients had a normal karyotype, while in six patients cytogenetic aberrations were identified. Interestingly 3/13 patients with PTPN11 mutations had FAB M5b morphology compared to only 12/413 PTPN11 neg. cases $(\mathrm{P}=.008)$ The CR rate was comparable in SHP-2 mutation positive and negative cases, however, the overall outcome of patients carrying this alteration was poor, with only 2 of the 13 patients being currently alive. In patients $<60$ years, cases with SHP-2 mutations had a significantly shorter event free survival (median 10.5 vs. 4.4. months; $\mathrm{p}=0.035$ ). Conclusion: Taken together, SHP2 mutations can be found in a subgroup of adult patients with AML. Consistent with the association with JMML, this mutation seems to more prevalen in patients with FAB M5b. Although very preliminary, the data point to a worse prognosis of patients with SHP-2 alterations. We are currently extending the dataset to further investigate the potential role of this aberration

\section{P551 \\ Identification of Methylation-Silenced Genes in Acute Myeloid Leukemia}

Agrawal S. ${ }^{1}$, Westermann A. ${ }^{1}$, Unterberg M. ${ }^{1}$, Wichmann M. ${ }^{1}$ Steffen B. ${ }^{1}$, Diederichs S. ${ }^{1}$, Hofmann W.K. ${ }^{2}$, Berdel W.E. ${ }^{1}$, Müller-Tidow K. ${ }^{1}$, Serve $H^{1}{ }^{1}$

${ }^{1}$ Department of Medicine, Hematology/Oncology, University of Münster, Germany, ${ }^{2}$ Dept. Of Hematology/Oncology, FU Berlin, Germany

Alterations of chromatin structure play a predominant role in the pathogenesis of acute myeloid leukemia (AML). These conformational changes are mainly caused by mechanisms like promoter methylation and histone acetylation. For example, methylation of tumor supressor genes such as p15 ranks among the most common aberrations found in AML. In addition, the translocation product PML-RARa has been demonstrated to induce methylation of the differentiation-associated RARbeta2 gene. However, no systematic knowledge exists, which specific genes are silenced by methylationdependent mechanisms as a consequence of fusion protein expression. Here we applied a microarray-based approach to screen for such genes. As a cell line model, we used U937 cells inducibly transfected with AML1-ETO or PML-RARa. In the absence of the fusion proteins, cells were exposed to the demethylating agent 5-azacytidine for 6 days. Genome wide changes in gene expression levels were analyzed by high-density microarray analyses. In a first step, we analyzed changes in the expression profiles before and after 5-azacytidine exposure. Overall, 118 genes were consistently induced at leas 5 -fold by demethylation treatment in the abcense of translocation products Among these were genes encoding S100 proteins, transcription factors and chemokines. More than $90 \%$ of the induced genes identified in the microarray analyses were confirmed by real-time RT-PCR. Bisulfite analyses for some of the identified genes verified CpG-island methylation of the genes' promotors in vivo, establishing the overall validity of the approach. Subsequently, the cells were released from 5-azacytidin, and the effects of the fusion proteins AML1-ETO or PML-RARa were studied. This approach allowed us to identify genes that were repressed by DNA methylation in a fusion-proteindependent manner. Here, several unexpected findings were obtained. For example, a myeloid transcription factor and growth suppressive genes were repressed by PML-RARa. Taken together, these data indicate that genes involved in differentiation and growth suppression are silenced by methylation in leukemia cell lines. Some of these genes were shown to be specifically repressed by AML fusion proteins, presumably by mechanisms involving DNA methylation. Thus, the approach provides a novel link between balanced translocations and specific alterations in DNA methylation patterns.
P552

Proteomic Identification of the C/EBPalpha Multiprotein

Complex Reveals that Downregulation of c-Jun N-Terminal Kinase 1 (JNK1) Activity Leads to loss of C/EBPalpha Function in Patients with Acute Myeloid Leukemia

Trivedi A.K. ", Bararia D. *, Peerzada A.A., Hiddemann W., Behre G. Medicine III at University of Munich Hospital Grosshadern, Munich, Germany ( ${ }^{*}$ authors contributed equally)

Acute myeloid leukemia (AML) is characterized by prominent myeloid blast and genetic aberrations hampering protein function. Functional inactivation of transcription factor $\mathrm{C} / \mathrm{EBPalpha}$ leads to $\mathrm{AML}$, whereas activation of $\mathrm{C} / \mathrm{EBP}$ alpha restores normal myeloid stem cell differentiation. We and others have shown that protein-protein interactions of C/EBPalpha plays a pivotal role in myeloid differentiation and AML. Systematic identification and characterization of proteins at a global proteome-wide level using proteomics has emerged as a novel tool. We hypothesized that the identification and functional characterization of all C/EBPalpha interacting proteins will lead to novel insights into the systems biology of C/EBPalpha. Thus, the GST-DNABinding domain of C/EBP was incubated with nuclear extracts of U937 cells, interacting proteins were identified by two-dimensional gel electrophoresis and MALDI-TOF mass spectrometry, and functionally characterized by bioinformatic pathway discovery with subsequent biological and mechanistic confirmation of the discovered pathway. We were able to identify known as well as other novel interacting proteins of C/EBPalpha which include p21 activated kinase 6 , MADP-1, binding protein, transcriptional coactivator sp110, and c-Jun N-terminal kinase 1 (JNK1). JNK1 binds to the C/EBPalpha DNA binding domain in GST-pull-down and in co-immunoprecipitation assays in vitro and in vivo, respectively. JNK1 phosphorylates and enhances the half life of C/EBPalpha protein. Thereby, JNK1 enhances the transactivation and DNA binding activity of C/EBPalpha which in turn leads to G0-G1 arrest in cell cycle. JNK mRNA expression as well as its kinase activity is low in AML patients which supports the hypothesis that C/EBP alpha might be inactive in AML because of lack of JNK1 activity. Thus, we propose JNK1 phosphorylates C/EBPalpha and stabilizes the half life of C/EBPalpha and thereby activates it. In AML, however, JNK1 activity is downregulated leading to a loss of C/EBPalpha function.

P553

\section{Loss of the Short Arm of Chromosome 17 in Patients with de Novo Acute Myeloid Leukaemia is Indicating Inferior Prognosis even in the Absence of Additional Cytogenetic Aberrations}

Mohr B., Illmer Th., Schaich M., Soucek S., Thiede C., Bornhäuser M., Ehninger G.

for the German multi-centre treatment trial of the SHG AML96 study group

Purpose: Loss of the short arm of chromosome 17 is a rare cytogenetic abnormality in patients with AML. A deletion of $17 \mathrm{p}$ can be of variable exten but usually involves the tumour suppressor gene p53 (Soenen et al. 1998) by allelic loss. The tumour suppressor protein p53 plays an important role in the control of genetic stability and it is well described that loss of p53 function contributes to malignant progression. In AML p53 inactivation is accompanied by chromosomal instability leading to complex aberrant karyotypes, where the outcome is poor. Results: Out of the 1331 patients with de novo AML included into the multicentric SHG-AML96 study 111 patients had a complex aberrant karyotype. 38 (34\%) of these patients were shown to have a loss of p53. There were only $12(1 \%)$ patients with a loss of $17 \mathrm{p}$ as a single aberration or accompanied by only one other abnormality. We compared the clinical course of patients with singular loss of p53 with patients showing instable karyotypes as defined by additional (multiple) aberrations. Five out of the 12 patients $(41,7 \%$ ) achieved CR after the double induction therapy of the SHG96 study including intermediate dose Ara-C (MAV-MAMAC). However, outcome was poor with 2 early relapses after 224 and 282 days, respectively, the other 3 patients succumbed due to early death. Generally, overall survival and event free survival of the 12 patients were inferior as compared to patients with a normal karyotype $(\mathrm{p}<0,001)$ and not different from patients with a complex aberrant karyotype. After the period of $2-417$ days (median survival 109,5 days) no patients was alive. Conclusions: In conclusion, patients with a loss of the short arm of chromosome 17 are a high- 
risk subgroup which does not depend on further cytogenetic aberrations Clearly, intergroup studies in this rare patient population are needed to further clarify best treatment options for patients with loss of this critical cell cycle check point.

\section{P554}

Risk Adapted Therapy in Acute Promyelocytic Leukemia: AIDA 2000 - A DSIL Study Group (Deutsche Studieninitiative Leukämie) Treatment Protocol

Schäkel U. ${ }^{1}$, Schuler U. ${ }^{1}$, Wandt $H_{.}{ }^{2}$, Thiede $C .{ }^{1}$, Schaich M., Ehninger . $^{1}$, on behalf of the DSIL

${ }^{1}$ Universitätsklinikum Dresden, Germany, ${ }^{2}$ Klinikum Nord Nürnberg, Deutsche Studieninitiative Leukämie (DSIL), Germany

Purpose: Since November 2000 we registered 41 patients ( 23 pat. $\leq 60$ ys, 18 pat. > 60 ys) with newly diagnosed acute promyelocytic leukemia (M3 / M3v $=33 / 8$ pat.). Based on different international studies we established a risk adapted treatment protocol according to age $(\leq 60 \mathrm{ys} />60 \mathrm{ys})$ and initial leucocyte count $(\leq />10 \mathrm{GPT} / \mathrm{l})$. The main objectives of the treatment protocol are to investigate 1 . the efficiency and toxicity of the treatment plan, 2 . the patients outcome with the prognosis dependent reduction of the intensity of consolidation therapy and whether the high risk group of patients seems to benefit from more intensive therapy, 3. the detection of kinetics of the PML-RAR $\alpha$ transcript for further therapeutical decisions and 4. correlating other pretreatment factors with the risk of relapse. Therapy: In the induction therapy ATRA $45 \mathrm{mg} / \mathrm{m}^{2} / \mathrm{d}$ until CR but not more than 90 days and idarubicin $12 \mathrm{mg} / \mathrm{m}^{2} / \mathrm{d}$ for 4 days were administered. The 1 . consolidation therapy consists of daunorubicin $60 \mathrm{mg} / \mathrm{m}^{2} / \mathrm{d}$ for 3 days in patients $\leq 60 \mathrm{ys}$, $45 \mathrm{mg} / \mathrm{m}^{2} / \mathrm{d}$ for 3 days in patients $>60$ ys and additionally Ara-C in patients with initially leucocytes $>20 \mathrm{GPT} / 1200 \mathrm{mg} / \mathrm{m}^{2} / \mathrm{d}$ as continuous infusion for 7 days in patients $\leq 60 \mathrm{ys}$ and $100 \mathrm{mg} / \mathrm{m}^{2} / \mathrm{d}$ in patients $>60 \mathrm{ys}$ ). As 2 . consolidation course mitoxantrone $10 \mathrm{mg} / \mathrm{m}^{2} / \mathrm{d}$ for 3 days and Ara-C in patients with initially leucocytes > $20 \mathrm{GPT} / 1$ twice daily $3 \mathrm{~g} / \mathrm{m}^{2} / \mathrm{d}$ for 4 days in patients $\leq 60$ ys and $1 \mathrm{~g} / \mathrm{m}^{2} / \mathrm{d}$ in patients $>60$ ys were administered. As maintenance therapy over 2 years patients are treated with 6-mercaptopurine daily $90 \mathrm{mg} / \mathrm{m}^{2} / \mathrm{d}$, methotrexate weekly $15 \mathrm{mg} / \mathrm{m}^{2} / \mathrm{d}$ and intermittend ATRA 45 $\mathrm{mg} / \mathrm{m}^{2} / \mathrm{d}$ over 15 days every 3 months. Results: At time of evaluation $90 \%$ (35/39 pat.) of all patients and $100 \%$ of patients $\leq 60$ ys achieved complete remission. 6 patients died ( 1 patient $\leq 60$ ys, 5 patients $>60$ ys) ,1 patient during induction therapy due to tumor lysis syndrome and haemorrhagic disorder, 4 patients after induction therapy due to sepsis, pneumonia and multiorgan failure, another during consolidation therapy due to sepsis. The overall survival was $85 \%$ in all patients, there was a significant difference in overall survival due to age (pat. $\leq 60$ ys: $95 \%$, pat. $>60$ ys: $72 \%, p=0,04$ ) No significant difference in survival was shown in patients with M3 / M3v leucocyte count $\leq />10 \mathrm{GPT} / \mathrm{l}, \mathrm{t}(15 ; 17)$ with or without other chromosoma abnormalities. 2 patients relapsed. Conclusion: Comparable to other trials these results indicate a high effectiveness in the treatment of acute promyelocytic leukemia.

\section{P555}

\section{Hyperdiploidy in Acute Leukemias and MDS is Associated with Poor Prognosis}

Balleisen S., Hildebrandt B., Haas R., Gattermann N., Germing U., Royer-Pokora $B$.

University Hospital Düsseldorf, Germany

Purpose: In order to determine the frequency and prognostic impact of hyperdiploidy, we reviewed 332 cases of acute myeloid leukemia (AML), 718 cases of myelodysplastic syndrome (MDS) and 214 cases of acute lymphoblastic leukemia (ALL) that were karyotyped and followed up at our institutions.Results: Hyperdiploidy ( $>48$ chromosomes) was found in 14 of $214(6,5 \%)$ patients with ALL. Twelve patients had massive hyperdiploidy with $\geq 50$ chromosomes (median 52, range 49-82). Three of those patients were also Philadelphia-chromosome positive. All 14 patients received induction chemotherapy. Complete remission (CR) was achieved by 11 patients, 9 of whom relapsed. Median survival was 13 months. Hyperdiploidy was present in 4 of $332(1,2 \%)$ patients with AML. Three had a massive hyperdiploidy (50, 54, and 77 chromosomes, respectively). Two of them achieved $\mathrm{CR}$, but both relapsed within three months. None of the patients is alive, and median survival was only 5 months. A hyperdiploid karyotype was docu- mented in 12 of $718(1.7 \%)$ patients with MDS. The diagnosis was refractory anemia (RA) in 4, and RAEB or RAEB-T in 8 patients. Seven patients had a massive hyperdiploidy (median 52, range 50 to 93 chromosomes). In 4 patients, the disease showed progression to AML. Among 5 patients who received induction chemotherapy 3 achieved CR but had an early relapse. Median survival in MDS patients with hyperdiploidy was 10 months (5-18). While it is known that hyperdiploidy is a complex karyotype, which is an important adverse risk factor in MDS and AML, the prognostic impact of hyperdiploidy in hematological malignancies is not well established. In childhood ALL, hyperdiploidy is found in up to $27 \%$ of patients and is associated with a favourable prognosis, which appears to be different in adult ALL. In myelodysplastic syndromes, we are not aware of any previous investigations addressing this issue. For patients with AML, Iyer et al recently reported that hyperdiploidy is associated with a poor prognosis. Conclusions: Our results indicate that hyperdiploidy is not a rare event in adult ALL, where it is associated with an unfavorable prognosis. Only two patients, both with T-ALL, remain alive, after 43 and 46 months of follow-up, respectively. Hyperdiploidy was rare in our patients with AML and MDS, but clearly associated with a poor outcome. In summary, we found that hyperdiploidy is an unfavorable prognostic factor in adult patients with hematological malignancies such as ALL, AML, and MDS.

\section{P556}

Prognostic Significance of FLT3 mRNA Expression in the Bone Marrow of Patients with Acute Myeloid Leukemia (AML) and its use as a Marker for Minimal Residual Disease (MRD)

\section{Kainz B. ${ }^{1}$, Fonatsch C. ${ }^{2}$, Schwarzinger I. ${ }^{3}$, Sperr W.R. ${ }^{1}$,} Lechner $K^{1}$, Valent $P .{ }^{1}$, Jäger $U^{1}{ }^{1}$, Gaiger $A$.

${ }^{1}$ Univ.-Klinik für Innere Medizin I, Abteilung Hämatologie,

Medizinische Universität Wien, Austria, ${ }^{2}$ Institut für Medizinische Biologie, Medizinische Universität Wien, Austria, ${ }^{3}$ Institut für Medizinische und Chemische Labordiagnostik, Medizinische Universität Wien, Austria

Based on its role in leukemia, FLT3 is an attractive molecular target for leukemia therapy and several potent inhibitors are currently being tested in AML patients with mutant FLT3 receptors. Studies demonstrating that FLT3 is overexpressed in AML and that FLT3 overexpression renders leukemic cells more sensitive to the therapeutic effects of FLT3 inhibitors indicate that the target could be extended to AML cases overexpressing FLT3 even without mutations We studied the expression of wild-type FLT3 mRNA at diagnosis and assessed its utility as a marker for minimal residual disease in AML. FLT3 mRNA expression in diagnostic bone marrow (BM) samples of 89 patients was analyzed by Real Time PCR. Compared to BM of healthy donors FLT3 mRNA was overexpressed in 88/89 AML patients (mean 30,3fold, range 0,6 - to 214-fold). High expression of FLT3 ( $\geq 41,5$-fold) was associated with worse overall survival $(P=0,02)$, the percentage of BM blasts $(P=0,03)$ and high WT1 expression $(P=0,002)$. No association between levels of FLT3 expression and disease free survival, clinical characteristics, cytogenetics, complete remission (CR) rate or relapse rate was observed. FLT3 mRNA expression in the BM was monitored during remission and/or at the time of relapse in 35 patients. In CR FLT3 mRNA expression decreased to levels similar to those of healthy donors and remained low in continuous $C R$ (0,5- to 4,6-fold). In contrast, FLT3 levels increased at the time of relapse (0,4- to 11,07-fold). The drop in FLT3 expression from diagnosis to CR was not significantly different between patients who stayed in remission or who relapsed. We conclude that expression of FLT3 mRNA has prognostic significance but is of limited value for monitoring of MRD. In this study, we show an influence of FLT3 mRNA overexpression on OS in patients with AML. However, there was no difference in DSF, which leads to the conclusion that patients with high FLT3 mRNA expression could be more resistant to relapse therapy and therefore might benefit from FLT3 inhibitors in consolidation and/or relapse therapy. 
P557

\section{Correlation of FLT3 Transcript Levels in Acute Myeloid Leukaemia with Clinical Parameters}

Kuchenbauer F., Kern W., Kohlmann A., Schoch C., Hiddemann W., Haferlach T., Schnittger S.

Laboratory for Leukemia Diagnosis, University Hospital Grosshadern, Munich, Germany

During the last years FLT3 mutations became important prognostic factors in acute myeloid leukaemia (AML). Considering the frequent expression of wild type FLT3 in leukaemic and normal bone marrow and its function as regulator of differentiation, apoptosis and modulation of the immune system, the role of the wild type FLT3 transcript level in adult AML is not fully understood. In the presented work FLT3 transcript levels of 186 AML and 7 healthy donors were assessd by real time PCR patients and correlated to cytogenetics, FAB, mutation status, as well as other clinical relevant factors. We found that FLT3 expression significantly correlated with the number of blasts $(\mathrm{p}=.005)$ and leukocytes $(\mathrm{p}=.003)$. FLT3 expression levels were not distrib uted equally within different FAB subtypes (M3 $<\mathrm{M} 2<\mathrm{M} 6<\mathrm{M} 4<\mathrm{M} 0$, M $<$ M5). Thus, the lowest median levels were assessed in FAB M3/M3v ( $=22$ and the highest mean levels in FAB M5a ( $\mathrm{n}=7)$. According to cytogenetic the lowest levels were detected in $\mathrm{t}(15 ; 17)$ and $\mathrm{t}(8 ; 21)$ and the highest in $\mathrm{t}(11 \mathrm{q} 23)$ and normal karyotype. Differences between the lowest $\mathrm{t}(15 ; 17)$ and highest expressers $\mathrm{t}(11 \mathrm{q} 23)$ were significant $(\mathrm{p}=.001)$. No difference could be shown within the group of secondary AMLs and therapy associated AMLs compared to idiopathic AMLs. In contrast to previous publications no difference of FLT3 expression could be detected between AMLs with FLT3 length mutations (FLT3-LM) $(n=52)$, FLT3 point mutations $(n=11)$ and without any FLT3 mutation $(\mathrm{n}=114)$. In addition, FLT3 mRNA expression as assesses by real time PCR was correlated to expression meassured by microarray analysis (Affymetrix) and FLT3 protein expression (CD135) as assess by flow cytometry and was found to significantly correlate with both parameters $(\mathrm{p}<.001$ and $p=.003$, respectively). Using Cox-regression analysis a significant influence of expression level to overall and event free survival could not be shown yet. Further analysis are needed to evaluate the importance of FLT3 expression to identify new possible risk groups, which might have implication on therapy stratification.

\section{P558}

\section{Protein Expression Profiling in Acute Myeloid Leukemia to Identify Therapeutically Significant Pathways: central role of the AP-1/c-MYC pathway in $t(8 ; 21)-A M L$}

Balkhi M.Y., Trivedi A., Christopeit M., Schnittger S., Hiddemann W., Behre G.

Department of Internal Medicine III, University Hospital Grosshadern, Ludwig-Maximilians-University Munich, Germany

In the present study we used a proteomic approach for an unsupervised protein cluster analysis for pathway finding in AML subtypes. The AML subtypes, M1, M2 t(8; 21), FLT3LM, M3 t (15; 17), M4, C/EBP alpha mutants, and ALL $t(4 ; 11)$ were used for the initial proteome profiling. With 2-D gel electrophoresis using $\mathrm{pH} 3-10$ in IEF and subsequent MALDI-TOF mass spectrometry, we were able to identify 50 proteins in each AML subtype. The proteins identified in M2 $\mathrm{t}(8 ; 21)$ include transforming protein Fos B, gene P27KIP1 protein, Ras-GRF2, MYC, p21CDC42Hs kinase, prohibitin, FUSE binding protein, TANK binding kinase, TBK1, and others The unsupervised protein cluster analysis using Ingenuity pathway finde software program showed the involvement of C- MYC and FOS pathways in M2 $t(8 ; 21)$ patient samples. This is in support of our previous finding that AP-1 transcription factors like c-JUN play an important role in leukemogenesis. To further validate these findings we used siRNA against the core protein involved in this pathway. These results suggest novel insights into the pathogenesis of AML and might lead to comprehensive molecular therapy against acute myeloid leukemia.

\section{P559}

Outcome of Patients with AML after Potential Curative Treatment at the Department of Hematology \& Oncology, Innsbruck

Lackinger G., Gunsilius E., Nachbaur D., Wolf D., Rumpold H., Oexle H., Erdel M., Ulmer H., Gastl G., Petzer A.L.

University Hospital Innsbruck, Austria

Purpose: We performed a retrospective analysis on 73 newly diagnosed patients (pts) with AML (74\% de-novo-AML), treated at our Institution during 1996 - 2002. Methods: The latest update was performed in Decembe 2002 with a median follow-up of 19 months. Results: Median age was 57 years. 46 patients were $\leq 60$ years and 27 pts $>60$ years. All pts received potentially curative treatment regimens. After first cycle of induction therapy $63 \%$ achieved CR ( $\leq 60$ yrs: $70 \%,>60$ yrs: $52 \%$ ), $10 \%$ PR and $16 \%$ had no response. Early-death rate was $9.6 \%$ ( $\leq 60$ yrs: $4.3 \%$, $>60$ yrs: $18.5 \%$ ). Median remission duration for all 73 pts was 9.6 months, the median overallsurvival (OS) was 19.1 months (cumulative-OS: $33 \%$ at 4 years). The median OS for patients $\leq 60$ yrs. was 22.3 months in comparison to 13.7 months for elderly patients $>60$ years. The OS at 4 years in patients $\leq 60$ years was almost twice as high as in elderly patients (39\% vs. $22 \%$ ). Within the group of elderly patients major differences in survival rates were seen. The majority of patients $\geq 65$ years relapsed early and their OS at 3 years was $16 \%(n=14)$ in comparison to $29 \%$ for patients between 61 and 64 years $(n=13)$. According to cytogenetic risk groups the patients $\leq 60$ years with low risk cytogenetics $(\mathrm{t}(8 ; 21), \mathrm{t}(15 ; 17)$, inv16) had a five-year-OS of $57 \%(\mathrm{n}=7)$, with intermediate risk $40 \%(n=27)$. So far no patient in the high risk group survived longer than 2 years; the cumulative-survival after one year was merely $13 \% \quad(n=10)$ Patients receiving at least one course of intermediate-dose-cytarabine during consolidation therapy $\left(1 \mathrm{~g} / \mathrm{m}^{2} / \mathrm{d}\right.$ over 5 days) or high-dose cytarabine $(\geq 2$ $\mathrm{g} / \mathrm{m}^{2} / \mathrm{d}$ over 5 days) performed better (OS $58.8 \%, \mathrm{n}=12$ and $42.2 \%, \mathrm{n}=27$ at 5 years, respectively) than patients receiving standard doses of cytarabine (100 $-200 \mathrm{mg} / \mathrm{m}^{2} / \mathrm{d}$ over 7 to 10 days; 3 -years-OS: $16 \%, \mathrm{n}=34$ ). The administration of high (or intermediate)-dose cytarabine instead of standard doses prior to alloSCT had no influence on OS. Conclusions: Our data suggest, that the application of intermediate and high doses of cytarabine definitely improve OS in patients not receiving an allotransplant. In contrast, the cumulative dose of cytarabine applied before alloSCT had no impact on the outcome of alloSCT. In addition, for most pts between 61 and 64 years intensive treatment with curative intent should be performed whereas for the majority of patients $\geq 65$ years intensive chemotherapy should be carefully considered to palliative treatment options or best supportive care.

\section{P560}

\section{Persisting Karyotyp Abnormalities after Induction Chemotherapy in AML and High Risk MDS Predict Poor Outcome}

Balleisen S., Hildebrandt B., Royer-Prokora B., Knipp S., Haas R., Gattermann N., Germing U.

University Hospital Düsseldorf, Germany

Purpose: Cytogenetic findings at diagnosis are of prognostic value to predic treatment outcome and long-term prognosis in patients with AML and MDS Therefore, treatment is stratified according to the karyotype. The purpose of our study was to assess the prognostic meaning of cytogenetic examination of bone marrow cells after induction chemotherapy in AML and high-risk MDS We examined 116 patients with AML $(n=103)$ or high-risk MDS $(n=13)$ with an abnormal karyotype at diagnosis, who received induction chemotherapy and whose karyotypes after induction were available. Initial karyotypes were as follows: $14 \times \mathrm{t}(15 / 17), 9 \times \mathrm{t}(8 / 21), 5 \mathrm{x}$ inv(16), 56 intermediate-risk, and complex karyotypes. The following induction protocols were used: $38 \mathrm{x}$ TAD, $4 \times$ HAM, $24 \times$ Ida-Ara, $36 \times$ ICE, and $3 \times$ other protocols. 11 patients received induction chemotherapy + ATRA. Results: 77 patients $(66 \%)$ achieved cytologic remission (CR), 20 patients achieved partial remission (PR), and 19 patients were non-responders. There were 26 out of 77 patients (33\%), who reached cytologic remission, but still showed an abnormal karyotype after induction. Median survival of these 26 patients was 23 months as compared to 52 months in the $51 \mathrm{CR}$ patients, who achieved both cytologic and cytogenetic remission (CCR). 17 of the 26 patients, who did not enter cytgenetic remission had been classified as standard-risk and therefore did not receive intensified consolidation. 24 of 28 patients with a low-risk kary- 
otype anomaly $((\mathrm{t}(15 / 15), \mathrm{t}(8 / 21)$, inv(16)) achieved cytogenetic remission The CCR rate was substantial lower in patients with intermediate-risk cytogenetic group (25/56) and in patients with complex karyotype (11/32). Median survival in the group achieving CCR was 42 months as compared to 13 months in patients with persisting abnormal karyotype after induction $(\mathrm{p}<0.00005)$. The difference remained highly significant when calculated only for patients with complex or intermediate-risk karyotype (median survival 31 vs 12 months). Conclusions: The examination of the karyotype after induction is useful to detect a persisting malignant clone and gives additional information to cytology. Achieving cytogenetic remission after induction therapy is strongly correlated with a better long-term outcome. Patients with a persisting abnormal karyotype can be recognised as high-risk patients and should therefore receive intensified treatment.

\section{P561}

Feasibility of Fast Search Diagnostics for AML Patients in a Multi-Center Study - First Experience within the DSIL AML2003 Trial

\author{
Schaich M., Füssel M., Bornhäuser M., Thiede C., Mohr B., \\ Illmer T., Schäkel U., Prange-Krex G., Oelschlägel U., \\ Ehninger G. on behalf of the DSIL AML2003 study group
}

Study Center: Med. Klinik I, Universitätsklinikum C.G.Carus, Dresden

Since December 2003 the AML2003-Study of the German Study Initiative Leukemia ("DSIL" former "SHG Dresden") is activated. It represents the follow-up study of the AML96 protocol for patients $<=60$ years. AML2003 is a prospective randomized trial, to investigate the value of an intensified treatment strategy, i.e. early allogeneic stem cell transplantation in aplasia after induction therapy, for high-risk patients. Therefore, a rapid analysis of risk factors (cytogenetics, FLT3 status and clearance of blasts after first induction) and the donor situation is of utmost importance. This "fast search diagnostics" together with routine analyses of morphology and immunophenotyping is accomplished centrally in all enclosed patients. Within the first 5 months 67 patients with a median age of 48 (17-60) years were included in the study. Fast search diagnostics including the information about possible related or unrelated donors was complete with a median of 14 days after arrival of the bone marrow samples for all patients. 39/67 patients were upfront randomized into the intensified treatment arms. Out of these $19(49 \%)$ patients with high-risk have been identified. Seven high-risk patients $(37 \%)$ received early allogeneic stem cell transplantation in aplasia after the firs $(n=3)$ or the second $(n=4)$ induction therapy course within the protocol. Three were transplanted with stem cells of related and four of unrelated donors. In three patients early allogeneic transplantation was not possible due to the absence of an available donor. One patient has not finished the first induction course yet. Eight patients showed refractory disease after the first induction course and were referred to salvage therapy. Two of them already received an allogeneic transplant.

These encouraging preliminary results show that fast search diagnostics is feasible in a large multi-center study. This leads to a significant proportion of early allogeneic stem cell transplants in aplasia after induction therapy within the group of high-risk AML patients.

\section{P562}

\section{Case Report: Detection of Minimal Residual Disease after Induction Therapy in Peripheral Blood Stem Cell Apheresis by Flow Cytometry and FISH in a Patient with} AML FAB - M7

\section{Schöndube D. ${ }^{1}$, Matylis A. , Ratei R. ${ }^{1}$, Einsele H. ${ }^{2}$, Schoch $C .{ }^{3}$, Haferlach $T^{3}{ }^{3}$, Karawajew L. ${ }^{1}$, Rhein P. ${ }^{1}$, Ludwig W.-D. ${ }^{1}$ ${ }^{1}$ Medizinische Klinik m.S.Hämatologie, Onkologie und Tumorimmunologie, Robert-Rössle- Klinik im HELIOS Klinikum Berlin, Charité Campus Buch, Germany, ${ }^{2}$ Eberhard-Karls-Universität Tübingen, Germany, ${ }^{3}$ LMU München Großhadern, Germany}

Detection of minimal residual disease (MRD) in peripheral blood or bone marrow is a critical factor for treatment stratification and prognosis for patients with AML in complete remission after induction chemotherapy. In recent years autologous peripheral blood stem cell transplantation (aPBSCT) after consolidation therapy has been used as maintenance therapy in AML patients but only few studies have addressed the issue of MRD-detection in aphaeresis products in these patients.
We report a 43 years old female with anaemia, mild thrombocytopenia and blasts in the peripheral blood. Immunophenotyping showed a blast population

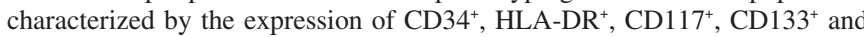
$\mathrm{CD} 61^{+}$so that AML FAB M7 was diagnosed. Bone marrow showed severe fibrosis. Cytogenetic analysis revealed a complex karyotype with a 7q31 deletion detected by FISH in $90 \%$ of blast cells. The patient was treated according to the AMLCG 99 - protocol with two induction courses of high dose Cytarabine and Mitoxantrone resulting in complete morphologic remission in peripheral blood, although bone marrow cytology was not representative due to persisting fibrosis. Allogeneic bone marrow transplantation would have been the therapeutic option of choice for this patient. But as no HLA identical donor was available we collected autologous peripheral stem cells (aPBSC) after induction therapy in order to perform high dose chemotherapy. PBSC aphaeresis has then been analysed with FISH and resulted to be negative for the described 7q31 deletion. Subsequently, the PBSC aphaeresis product was sorted by flow cytometry based on CD34 expression and FISH was performed again on $\mathrm{CD}^{+} 4^{+}$sorted cells. $7 \mathrm{q} 31$ deletion could now be clearly detected, demonstrating the persistence of the initial leukemic clone. We therefore decided to perform a haploidentical PBSCT with one brother as donor. Complete remission was achieved without important signs of GVHD (grade I). One year after transplantation the patient is still in complete remission and well.

Conclusions: In high-risk AML patients planned to receive aPBSCT, the combination of flow cytometry and cell sorting according to the initial leukaemia- associated immunophenotype in the aphaeresis product and FISH for known chromosomal aberrations, represents a useful tool to detect residual blasts and avoid re-infusion of leukemic cells.

\section{P563 \\ Cytarabine based Regimens in Acute Myeloid Leukemia: - Assessment and Comparability of Treatment Intensity - Use of the Concentration Coefficient Weighted Area under the Curve (N-AUC)}

\section{Braess J., Fiegl M., Zimmermann I., Lorenz I., Hiddemann W. Klinikum Grosshadern, Munich, Germany}

Background: Current approaches to improve the results of antileukemic treatment against AML usually involve "treatment intensification". However rational treatment intensification requires knowledge about the parameter that is to be intensified. I.e. there must exist a clearly established relation between that particular parameter and treatment effect (e.g. CR rate). A correlation of such parameters such as total drug dose, dose intensity or area under the curve (AUC) (esp. of cytarabine) and treatment effect has so far never been systematically been demonstrated in AML. Esp. AUC is often considered to best characterize an applied regimen, however it does not take into account the relative relevance of concentration versus exposure time for antileukemic efficacy. This shortcoming may be overcome by weighing the AUC by the so called concentration coefficient $\mathrm{N}$ which can be derived from the paradigm "CexpN·T = constant" for equitoxic effects (termed N-AUC). Purpose: 1) To establish the concentration coefficient $\mathrm{N}$ for cytarabine in AML. 2) To extrapolate from published pharmacokinetic analyses of cytarabine in AML the average AUC in each arm of a large set of randomized studies. 3) To demonstrate from these studies the existence of a linear relationship between response (CR rate) and cytarabine dose, dose intensity, AUC, N-AUC. Methods: 1) $\mathrm{N}$ was derived from in-vitro experiments that involved measuring equitoxic effects (LC-50\%) at varying exposure times in $54 \mathrm{AML}$ samples. 2) Pharmacokinetic parameters of diverse cytarabine regimens (doses ranging from 0,02 to $6,0 \mathrm{~g} / \mathrm{m}^{2} / \mathrm{d}$; exposure times ranging from 0,5 to $24 \mathrm{~h}$ per day) were extracted from the literature. 3) Randomized cytarabine based studies since 1990 were indentified in the literature, in which total dose, dose intensity and CR rate could be identified. Results: 1) (Equi)Antileukemic effects of cytarabine were well described by the paradigm "CexpN.T = constant", which therefore made the derivation of $\mathrm{N}$ possible: 0,45 - N.B. that a concentration coefficient $<1$ implies a higher relevance of exposure time for increased effects as compared to concentration. 2) Published cytarabine AUCs are highly linear to dose and are not affected by the infusion time, which therefore made plausible the assignment of average AUCs to the various treatment arms of other AML studies $(n=50)$ in which only doses and infusion times were stated. 3) No relationship could be found between $\mathrm{CR}$ rate and cytarabine dose $(\mathrm{r}=0,1211, \mathrm{p}=0,4122)$, dose intensity $(\mathrm{r}=0,1388, \mathrm{p}=0,3366)$ or AUC $(\mathrm{r}=0,1068, \mathrm{p}=0,4955)$. However there was a strong linear correlation between CR rate and N-AUC $(r=0,5901, p<0,0001)$. 
Conclusions: 1) We established the concentration coefficient $\mathrm{N}$ of cytarabine in AML, which is a useful parameter to formally quantify the relative relevance for the clinical effect of exposure time versus concentration. 2) N-AUC of cytarabine is highly correlated with the clinical effect, which suggests that it might be a better parameter for "treatment intensification" than dose, dose intensity or AUC. 3) The concept of N-AUC allows the realistic comparison of pharmacokinetically widely diverse applications of cytarabine and immediately suggests rational ways of treatment intensification.

\section{P564 \\ The Bisphosphonate Zoledronate Shows Cytotoxic Effects in AML, but is Not Synergistic with Cytarabine}

Jürgens M., Fiegl M., Heil K., Hiddemann W., Braess J.

Department of Internal Medicine III, Ludwig-Maximilians-University Munich, Germany

Purpose: The third generation bisphosphonate Zoledronate (ZOL) (Zometa ${ }^{\circledR}$, Novartis), a heterocyclic imidazole, has been shown to exert antitumorous activity in various cell lines including breast cancer, multiple myeloma and chronic myeloid leukemia. The main mechanism of action is supposed to be inhibition of ras protein signaling by inhibition of both farnesylation and geranylgeranylation. In this study we investigated the antileukemic activity of single ZOL and in combination with Cytarabin in Acute Myeloid Leukemia (AML). Methods: HL60 cell lines $(n=5)$ and fresh blasts $(n=4)$ from patients with AML were studied. For assesment of cytotoxicity and possible interactive effects of ZOL and AraC the WST-1 assay was used. The assay is based on cleavage of the tetrazolium salt WST-1 to a coloured formazan dye by mitochondrial dehydrogenase of viable cells. This signal can be detected spectrophotometrically and directly correlates to the number of viable cells. The assay was performed in 96 well plates with increasing concentrations of ZOL and AraC over 24 hours. All experiments were carried out at least in triple for HL60. Interactions were assessed by isobologram analysis. Results: ZOL shows significant antileukemic activity in HL60 cell lines and AML blasts. Mean LC50 (lethal concentration for $50 \%$ of cells) in HL60 cells was $222 \mu \mathrm{g} / \mathrm{ml}$ after 24 . In cells from patients the mean LC50 was $606 \mu \mathrm{g} / \mathrm{ml}$ (range: 366.8 - $855.5 \mu \mathrm{g} / \mathrm{ml} ; \mathrm{n}=4$ ) after 24 hours. Using isobologram-analysis for evaluation of interactions, coincubation of $\mathrm{ZOL}$ with $\mathrm{AraC}$ for 24 hours in HL60 model could be shown to result in subadditive cell kill Conclusions:These data show that ZOL is active in HL60 cells as an AML modell but also in primary AML blasts in vitro. Although the incubation of ZOL alone resulted in substantial cell kill, no synergistic but rather subadditive effect interactions with AraC were observed in HL60 cells.

\section{P565}

Treatment of Acute Myeloid Leukemia (AML) in patients above the age of 60 years: A report of the AML97\#38 study of the East German Hematology and Oncology Study Group

Becker C., Krahl R., Al Ali H., Pönisch W., Wedding U., Fiedler F., Pasold R., Freund M., Subert R., Kämpfe D., Meran J., Herold M., Peter N., Knauf W., Kettner E., Schultze W., Dachselt K., Wolf H.H., Schott G., Schulze M., Ittel T., Assmann M., Grobe N., Huhn R., Steglich J., Schirmer V., Klinkenstein C., Doepper J., Winkelmann C., Helbig W., Niederwieser D.

For the East German Hematology and Oncology Study Group (OSHO), Germany

Purpose: Patient selection still plays an important role in curative treatment trials of elderly patients with AML. In the present AML97 study all patient above the age of 60 years with AML were reported and treated using either a curative, palliative or supportive protocol. Methods: Since March 1998 total of 520 patients were enrolled in this protocol (curative 375, palliative 112 and supportive 33 ). In the curative arm patients were treated with one or two courses of induction therapy (AraC $2 \mathrm{~g} / \mathrm{m}^{2}$ iv on day 1,3,5,7 and mitoxantrone $10 \mathrm{mg} / \mathrm{m}^{2}$ iv day 1-3) followed by 2 consolidation courses (AraC 240 $\mathrm{mg} / \mathrm{m}^{2}$ iv day $1-5$ and mitoxantrone $10 \mathrm{mg} / \mathrm{m}^{2}$ iv day $\left.1-2\right)$. Palliative treatment consisted of idarubicin $10 \mathrm{mg}$ po day 1 in combination with either thioguanine $40 \mathrm{mg}$ po day $1-5$, or AraC $80 \mathrm{mg}$ sc day $1-5$ or etoposide $100 \mathrm{mg}$ po day 1-5. Results: Cytogenetics at diagnosis was the most important prognostic factor for $\mathrm{CR}(\mathrm{p}<0,0005$, multivariate analysis). $93 \%$ of the patients with favourable, $80 \%$ with normal, $79 \%$ with other aberrations and only $46 \%$ with unfavourable karyotype achieved CR. The EFS at 2 years was $0,39 \pm$ 0,14 (median $463 \mathrm{~d}$ ), 0,24 \pm 0,05 (median $240 \mathrm{~d}$ ), 0,18 \pm 0,07 (median $206 \mathrm{~d}$ ) and 0,08 $\pm 0,04$ (median $84 \mathrm{~d}$ ) for patients with favourable, normal, other and unfavourable cytogenetics respectively. Furthermore with a median follow up of 283 days (range $33-1688$ ) the actual OS was $0,58 \pm 0,15$ (median $762 \mathrm{~d}$ ), $0,38 \pm 0,06$ (median $421 \mathrm{~d}$ ), 0,39 $\pm 0,09$ (median $411 \mathrm{~d}$ ) and $0,14 \pm 0,05$ (median 163) for patients with favourable, normal, other and unfavourable cytogenetics respectively. The median survival for patients treated with palliative Therapy was 54 days and only 12 days for patients treated with supportive care. Conclusions: Our used curative arm of the protocol is able to induce $\mathrm{CR}$ in a high proportion of elderly patients with AML with an acceptable early death rate. Despite high CR rate, EFS is still low. The treatmen results with palliative chemotherapy are disappointing. These results sugges the need to develop novel therapeutic strategies for these elderly patients and a risk-adapted treatment. To improve the EFS a new AML phase III study will start in July 2004 within the "Competenznetzwerk - Acute leukemia", One aim is the improvement of consolidation therapy by using low dose TBI based conditioning regimen and immunosuppression with cyclosporine and MMF followed allogenic PBSCT (MRD or MUD) or application of more intensive consolidation chemotherapy.

\section{P566 \\ High-Dose Melphalan is an Effective Salvage Therapy in Patients with Acute Myeloid Leukemia who Relapse after an Autograft}

\section{Bug G. ${ }^{1}$, Atta J. ${ }^{1}$, Klein S. ${ }^{1}$, Hertenstein B. ${ }^{2}$, Bergmann L. ${ }^{1}$, Böhrer S. ${ }^{1}$, Hoelzer D. ${ }^{1}$, Martin H. \\ ${ }^{1}$ Medizinische Klinik III, Klinikum der J.W. Goethe-Universität Frankfurt, Germany, ${ }^{2}$ Abt. Hämatologie/Onkologie, Medizinische Hochschule Hannover, Germany}

Purpose: Recurrent disease remains the most frequent reason for treatment failure in patients with acute myeloid leukemia (AML). In our center, salvage chemotherapy includes mitoxantrone, topotecan and cytarabine (MTC, L. Bergmann et al., Onkologie 2003, 26 (5), 86). However, complete remission (CR) rates are below $50 \%$ and overall long-term survival is poor. Attempts to intensify salvage regimens have lead to increased toxicity, but limited data are reported on the use of high-dose chemotherapy followed by an autologous peripheral stem cell transplantation (auto-PBSCT) in patients with untreated relapse. Methods: We therefore applied high-dose melphalan (HD-Mel, 140 $200 \mathrm{mg} / \mathrm{m}^{2}$ ) and auto-PBSCT to eight patients (median age 57, range 46-60 years) with recurrent AML: 2/8 patient had relapsed after conventional chemotherapy and 6/8 after auto-PBSCT performed in previous CR. At the time of HD-Mel salvage therapy, $2 / 8$ patients were in untreated relapse, while 6/8 patients had received one course of MTC 27-41 days prior to HD-Mel, leading to subsequent $\mathrm{CR}$ in one patient only. Five patients proved to be refractory to MTC. Results: All 8 patients, including those not responding to MTC, achieved CR after salvage therapy with HD-Mel and auto-PBSCT. Median cumulative duration of neutropenia was $>6$ weeks in the 5 patients refractory to MTC but only 2 weeks in patients with untreated relapse. CR lasted 2-7 months, thereby facilitating further allogeneic PBSCT: 5 patients proceeded to a matched unrelated donor (MUD)-PBSCT after 1.7-3.1 months. Two of five patients remained in CCR after 6 and 28 months and 3/5 died after MUD-PBSCT in CR due to infection and/or GvHD. Three patients did not receive a consolidating allogeneic PBSCT, relapsed again after 2, 5 and 7 months and eventually died. Conclusions: Our results suggest, that HD-Mel can safely be administered to intensively pretreated patients. It may induce second or subsequent remission even in patients refractory to conventional chemotherapy regimens with reasonable toxicity and a low rate of infectious complications, thereby facilitating allogeneic PBSCT in the majority of patients. Thus we propose to use HD-Mel as first-line salvage therapy in relapsed AML patients with stored autologous stem cells. 
P567

Treatment of Acute Myeloid Leukemia (AML) in Patients Below and Above the Age of 60 Years: Influence Factors for CR and Hematological Recovery. Study of the East German Hematology Oncology Study Group (OSHO)

Becker C., Krahl R., Al Ali H., Moosmann P., Pönisch W., Wedding U., Herold M., Subert R., Freund M., Fiedler F., Pasold R., Peter N., Meran J., Fasshauer E., Dachselt K. Grobe N., Assmann M., Kettner E., Steglich J., Huhn R., Schulze M., Schultze W., Ittel T., Klinkenstein C., Doepper J., Winkelmann C., Knauf W., Helbig W., Niederwieser D. For the East German Hematology Oncology Study group (OSHO), Germany

Purpose: The OSHO performed AML - phase III study's in patients $<60$ years (AML 96) and > 60 years (AML97). Methods: All patients were treated with one or two courses of induction therapy (AraC $2 \mathrm{~g} / \mathrm{m}^{2}$ iv on day $1,3,5,7$ in combination with idarubicine $12 \mathrm{mg} / \mathrm{m}^{2}$ day 1-3 in AML 96 or mitoxantrone $10 \mathrm{mg} / \mathrm{m}^{2}$ iv day $1-3$ in AML 97) followed by 2 consolidation courses. Consolidation courses were different in both study's. Due to identical chemotherapeutical regimes for younger and elderly patients, we analysed the whole patient population regarding $\mathrm{CR}$ rates and hematological recovery of leukocytes and platelets after induction therapy. Altogether we analysed 522 patients, 323 below and 209 above the age of 60 years. We performed a multivariate analyse (logistic regression model) to identify factors of influence for $\mathrm{CR}$ rate. We checked cytogenetics at diagnose, classi-

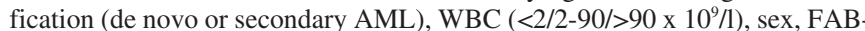
classification (FAB-M0/M1/M2/M3/M4/M5/M6/M7) and age. Results: The most important and highly significant parameter for $C R$ rate was cytogenetics at diagnose $\left(\mathrm{p}=10^{-10}\right)$. Classification (de novo or secondary AML) was significant $(\mathrm{p}=0,0001)$ too. In contrast, we could not demonstrate any significant influence of age $(\mathrm{p}=0,267)$. Significant factors for the recovery of leukocytes were cytogenetics $\left(\mathrm{p}=10^{-4}\right), C R$ yes/no $(\mathrm{p}=0,025)$ and classification (de novo or secondary AML $)(\mathrm{p}=0,034)$. There was no influence of age $(\mathrm{p}=0,9)$. Regarding platelets recovery we identified the following factors to be significant: CR yes/no $\left(\mathrm{p}=10^{-4}\right)$, classification (de novo or secondary AML $)\left(\mathrm{p}=10^{-4}\right)$ and cytogenetics $(\mathrm{p}=0,0014)$. There was no influence of age too $(\mathrm{p}=0,85)$. Conclusions: Cytogenetics at diagnose was the most important parameter for $\mathrm{CR}$ and highly significant in the performed multivariate analyse. We could not demonstrate any influence of age regarding CR rate and hematologica recovery. Although this is a retrospective analyses only of an induction therapy, these data may suggest that the biology of AML below and above the age of 60 years is not as different as described before.

\section{P568}

Prognosis of Adult Patients with CBF Leukemias a Single-Center Experience

\section{Mikesch K., Kunzmann R., Lübbert M.}

Dept. of Hematology and Oncology, University Medical Center Freiburg, Germany

CBF leukemias (carrying the AML1/ETO or CBFß/MYH11 rearrangement) belong to subgroups of acute myeloid leukemia (AML) characterized by a distinct genotype/phenotype relationship.

Table 1: Patient characteristics

$\mathrm{t}(8 ; 21): \mathrm{n}=14$ (5 with additional chromosomal abnormalities)

$\operatorname{inv}(16): \mathrm{n}=11$ ( 2 with additional chromosomal abnormalities)

\begin{tabular}{|c|c|}
\hline Median age (years) (range) all pts & $41(21-70)$ \\
\hline Male: female $(\%)$ & $16: 9(64: 36)$ \\
\hline Preceding MDS (\%) & $5(20)$ \\
\hline Chloroma at AML diagnosis (\%) & $2(8)$ \\
\hline Median WBC $(/ \mu 1)$ of all pts / $t(8 ; 21) / \operatorname{inv}(16)$ & $16050 / 8250 / 42200$ \\
\hline Median LDH (U/l) of all pts / $t(8 ; 21) / \operatorname{inv}(16)$ & $506 / 394 / 635$ \\
\hline Median bone marrow blasts (\%) all pts / t $(8 ; 21) / \operatorname{inv}(16)$ & $77 / 65 / 88$ \\
\hline Median peripheral blood blasts (\%) all pts / $\mathrm{t}(8 ; 21) / \operatorname{inv}(16)$ & $52 / 31 / 78$ \\
\hline
\end{tabular}

They are frequently associated with a favorable prognosis, particularly after high-dose cytarabine (HiDAC) consolidation, showing among all non-M3 AML a high response rate and often long remission durations. However, very few single-center studies have reported the outcome of CBF leukemias in unselected patients. Between June 1993 and October 2003, 25 consecutive adult patients (pts) with CBF leukemias were treated at our center. 23 pts received 1-2 induction courses with either IVA (16 pts, Heil et al., Ann Hematol. 2004, 83:336-334), or other induction protocols. 1 additional pt (35 yr) with inv(16) died of hyperleukocytosis and massive DIC prior to induction, 1 other pt $(70 \mathrm{yr})$ with $\mathrm{t}(8 ; 21)$ received low-dose Decitabine. 15/23 pts $(65 \%)$ achieved CR. Induction death occurred in $3 / 23$ pts $(13 \%)$, due to hyperleukocytosis in $1 \mathrm{pt}(25 \mathrm{yr})$ with inv(16), due to sepsis in 2 others. 5/23 pts did not achieve remission after two courses of induction chemotherapy, 4 of them were allografted. $11 / 15 \mathrm{CR}$ pts $(73 \%)$ received consolidation with HiDAC. Median overall survival was 19 months (mths). Median relapse-free survival was $12.8 \mathrm{mths}$ with a relapse rate of $33 \%$. 12/25 pts (48\%) are still alive with 4 pts $(16 \%)$ living more than 4 years, 3 of them having received HiDAC. Conclusion: This single-center analysis confirms that long-term remissions can be achieved in cytogenetically defined subgroups of AML using HiDAC consolidation with allografting being indicated for non-responders. We noted an almost 5-fold higher median WBC in inv(16) compared to $\mathrm{t}(8 ; 21)$ pts in 2 cases associated with fatal, early hyperleukocytosis complications.

\section{P569}

\section{Myeloblasts in Acute Myeloid Leukemia (AML) Produce and Secrete an Active Form of Tryptase}

\section{Sonneck K., Florian S., Hauswirth A.W., Sperr W.R., Valent P.} University Hospital Freiburg, Germany

Purpose: Several blast-derived growth regulators are considered to be involved in the pathogenesis of acute myeloid leukemia (AML). Tryptase is a highly potent mitogen for mesenchymal cells that has recently been implicated in fibroblast proliferation and angiogenesis. We have recently shown that myeloblasts produce significant amounts of tryptase in a group (roughly $40 \%$ ) of patients with AML (Sperr et al, Blood 98:2200, 2001). Methods: In the present study we asked whether soluble and functionally active tryptase is secreted by AML blasts and could serve as a relevant molecule in the pathogenesis of AML. Results: In all patients with tryptase+ AML examined $(n=5)$, the tryptase protein determined by FIA, was found to accumulate over time in supernatants of isolated unstimulated blast cells in vitro. Serum samples in these patients contained high tryptase concentrations $(>50 \mathrm{ng} / \mathrm{ml}$; normal range $<15 \mathrm{ng} / \mathrm{ml}$ ) and were found to promote uptake of ${ }^{3} \mathrm{H}$-thymidine in cultured bone marrow fibroblasts in a similar way compared to bFGF or recombinant human tryptase. To elucidate the possible role of tryptase in the pathogenesis of AML, we screened for expression of the tryptase receptor PAR2 using a monoclonal anti-PAR2 antibody. As determined by multicolor flow cytometry, PAR2 was found to be expressed on blood monocytes and the AML cell line KG1. In addition, myeloblasts in three patients with AML were found to react with anti-PAR2 antibody. Conclusions: In summary, our data show that AML blast cells in tryptase+ AML constitutively produce and release a functionally active form of tryptase. Whether blast cell-derived tryptase acts as an autocrine or/and paracrine growth regulator in patients with AML remains to be determined.

\section{P570}

\section{Chemotherapy-Induced t-MDS/AML-Associated Genetic Aberrations}

Bäsecke J., Podleschny M., Goldstein J., Riechel C., Haase D., Viereck V., Glass B., Trümper L., Griesinger F.

Dpt. of Hematology and Oncology and Dpt. of Gynecology and Obstetrics, University of Goettingen, Goettingen,Germany

Purpose: Therapy-related solid and hematological neoplasias occur according to the treatment regimen and intensity. The cumulative incidence of therapy related myelodysplastic syndromes and acute myeloid leukemias(t-MDS/AML) ranges between $5-15 \%$ in non-myeloablative and myeloablative treatment protocols respectively. t-MDS/AML share characteristic genetic aberrations which include translocations (e.g. involving 11q23, MLL and $\mathrm{t}(9 ; 22)$, inv $16, \mathrm{t}(8 ; 21), \mathrm{t}(3 ; 21), \mathrm{t}(15 ; 17)$ and cytogenetic aberrations 
(e.g. 5q-, 7q-). We have investigated if these genetic aberrations are present in patients which underwent chemotherapy. Since we and others have detected aberrations in aleukemic hematopoiesis (Bäsecke et al. Blood 2002;100:2267-2268) we also wanted to determine if t-MDS/AML early recognition can be performed by this screening. Methods: We enrolled patients with Non Hodgkin Lymphoma, either treated by conventional or high dose chemotherapy. Samples were taken as fresh peripheral blood stem cells (PBSCT), bone marrow aspirates (BM) or peripheral blood (PB). PBSC of healthy adult donors and cord blood of healthy newborn were used as control. Blood samples were submitted to RT- or Real-Time PCR (sensitivity 10-4 to 10-5) for $\mathrm{t}(9 ; 22)$, inv $16, \mathrm{t}(8 ; 21), \mathrm{t}(15 ; 17)$ and MLL-partial tandem duplications (MLL-PTD). Cytogenetic analysis was performed by FISH (probes EGR1-del5, p53, cep7-del 7, MLL and cep8 -tris 8) Results: In the cord blood control group, positive samples were $\mathrm{t}(15 ; 17) 1,7 \%$ (1/60 samples), inv $165,6 \%$ (3/54), $\mathrm{t}(8 ; 21) 2,8 \%(1 / 36), \mathrm{t}(9 ; 22) 3,4 \%$ (2/59), MLL-PTD primary PCR $0 \%(0 / 34)$. No positive results were observed in PBSC samples of healthy donors (22 samples). 53 samples of patients of which 21 were PBSC and 32 were BM or PB have been investigated so far. $34 / 53$ patients $(64,2 \%)$ underwent a high dose therapy. In $9,4 \%(5 / 53)$ the translocation $\mathrm{t}(15 ; 17)$ was detected. All five samples were PBSC. No amplification of inv $16, \mathrm{t}(8 ; 21)$ and $\mathrm{t}(9 ; 22)$ was observed. In FISH analysis two of 18 patient PBSC samples (11\%) exhibited aberrations in the p53 locus, which were classified as abnormal but still non-clonal. Conclusions: Our results confirm the existence of AML-associated translocations in cord blood at frequencies, which by far exceed the incidence of AML in healthy individuals. Compared to these findings the incidence of t-AML- associated translocations in patients which underwent chemotherapy-induced genotoxic stress is lower than expected. Positive PCR-results of this group may thus be more informative concerning the detection of a leukemic clone and the risk of attracting a t-MDS/AML. Positive patients are currently under observation and the number of samples and aberrations in the ongoing study is increased.

\section{P571}

Detection of Functionally Active Granulocytes in AML Patients Receiving Intermediate or High Dose ARA-C for Consolidation

Krauth M.T., Florian S., Hauswirth A.W., Samorapoompichit P., Sperr W.R., Valent $P$.

University Hospital Vienna, Austria

Purpose: High dose- (HiDAC) and intermediate dose- (IDAC) ARA-C have recently been introduced as effective post remission treatment for pts with AML. These regimens have been reported as a safe approach with a relatively low rate of infections and a relatively short time of absolute neutropenia. Methods/Results: In this study, we analyzed the numbers, phenotype, and functional properties of neutrophils in AML pts during consolidation with HiDAC $(n=8)$ or IDAC $(n=8)$. We found that absolute neutrophil counts (ANC) remained at relatively high levels until day 10-14. In a majority of the pts receiving IDAC or HiDAC, ANC on day 10 were $>500 / \mu 1$. These cells were found to be mature neutrophils by Wright-Giemsa staining and electron microscopy (EM). They contained secondary granules and phagolysosomes similar to neutrophils in healthy controls. In addition, these cells exhibited oxidative burst activity and expressed phagocytosis-related and activationlinked cell surface antigens including the C3biR (CD11b), CR1 (CD35), C5aR (CD88), Fc $\gamma R I$ (CD64), Fc $\gamma$ RII (CD32), Fc $\gamma$ RIII (CD16), G-CSF-R (CD114), GM-CSF-R $\alpha$ (CD116), and sLx (CD15). The biologic significance of neutrophils persisting until day 10 in AML pts was confirmed in functiona analyses using fluorescence labeled, heat-killed E.coli bacteria, flow cytometry, and EM. In these experiments, day 10 neutrophils were found to take up bacteria into phagolysosomes in the same way as neutrophils in healthy controls. Conclusions: In summary, our data show that considerable numbers of functionally active neutrophils survive until day 10 in AML pts treated with HiDAC or IDAC. The consecutive delay in occurrence of neutropenia may be responsible for the relatively low rate of severe infections in these pts compared to other consolidation regimens.
P572

Making the Diagnosis in Acute Leukaemia: Role of Peripheral Blood Smear, Bone Marrow Aspirate, Immunophenotyping of Peripheral Blood and Marrow Aspirate, and Bone Marrow Trephine Biopsy

\author{
Weiss B., Hoffmann M., Claus B., Bohrer M., Hellstern P., \\ Uppenkamp $M$. \\ Klinikum Ludwigshafen, Germany
}

Purpose: Which diagnostic procedures contribute rapidly and reliably to confirming acute leukaemia. Methods: From 05/2000 to 5/2004 82 patients were diagnosed with acute leukaemia in our institution (20 to 90 years of age, median 59 years). 67 patients were diagnosed with AML, 13 with ALL, one with plasmacell-leukaemia, and one with blastic transformation of Sezary syndrome. Initial haematological work-up enclosed peripheral blood smears, bone marrow aspirate and trephine biopsy with immunocytochemistry, and immunophenotyping of peripheral blood and bone marrow aspirate. Results: In 37 out of 67 patients AML could be confirmed by peripheral blood smears and immunophenotyping. 13 patients presented with leukocytosis and blast counts of less than 20\%. 15 patients displayed leukopenia, but 4 of these patients showed blast counts $>20 \%$, confirming AML. 2 patients presented with a normal blood count. Bone marrow aspirate and cytochemistry was applicable in 58 out of 67 patients confirming AML in 52 patients and revealing AUL (acute undifferentiated leukaemia) in6 cases. 2 patients had punctio sicca. Immunophenotyping was diagnostic in 55 patients and not evaluable in 3 patients. Bone marrow trephine biopsy was evaluable in 57 patients confirming AML in 49 and displaying AUL in 7. Major differences in diagnosis were noted with respect to bone marrow aspirates and immunophenotyping in 6 patients (myelodysplastic or myeloproliferative syndrome, acute lymphoblastic leukaemia, high grade - lymphoma). In ALL work-up of blood count, blood smear, and immunophenotyping was diagnostic in 7 patients, 3 patients presented with leukopenia/pancytopenia, and 3 patients displayed normal blood counts. Bone marrow aspirate and cytochemistry confirmed ALL in 6 patients, showed AUL in 5, and was misleading or not diagnostic in 2. Bone marrow trephine biopsy was applicable in 12 patients and compatible with ALL in 9 patients, revealing AUL in 2, and showing bone marrow contamination $<20 \%$ from lymphoblastic lymphoma in one. Careful work-up of peripheral blood smear confirmed AML in 61\% $(41 / 67)$ and ALL in 54\% (7/13). In suitable patients, combining blood smear and immunophenotyping of peripheral blood rendered AML in $87 \%(42 / 48)$ and ALL in $78 \%$ (7/9). Bone marrow aspirate and cytochemistry confirmed AML in $87 \%(52 / 60)$ and ALL in $46 \%$ (6/13) only. In ALL, combination of bone marrow aspirate and immunophenotyping was diagnostic in $92 \%$ (11/12). Bone marrow trephine biopsy confrmed AML in $86 \%(49 / 57)$ and ALL in $75 \%$ (9/12), and was misleading in 10\% (6/57). Conclusions: Bone marrow aspirate in combination with immunophenotyping of marrow cells and/or peripheral blood cells confirm rapidly and reliably acute leukaemia.

\section{Poster Session: Non-Hodgkin's Lymphoma I}

\section{P573}

Purine Antagonists for Chronic Lymphocytic Leukemia: Preliminary Results of a Comprehensive Meta-Analysis

\section{Steurer M. ${ }^{1}$, Pall G. ${ }^{2}$, Schwarzer G. ${ }^{3}$, Bohlius J., Greil R. ${ }^{5}$}

${ }^{1}$ Division of Hematology \& Oncology, University Hospital Innsbruck, Austria, ${ }^{2}$ Division of General Internal Medicine, University Hospital Innsbruck, Austria, ${ }^{3}$ Institute for Medical Biometry and Medical Informatics, University of Freiburg, Germany, ${ }^{4}$ Cochrane Haematological Malignancy Group, Köln, Germany, ${ }^{5}$ Third Medical Department, St. Johann Hospital / Paracelsus Private Medical University, Salzburg, Austria

Purpose: Recent trials suggest improved response rates for purine antagonists compared to alkylator-based regimens in the treatment of CLL. However, none was able to show a survival advantage, which may be due to study design or sample size. The aim of this study was to determine, if there is any advantage of purine antagonists compared to alkylating agents (alone or in combination) in the treatment of patients (pts) with CLL. Methods: Randomised controlled trials comparing purine antagonists with alkylatorbased regimens in pts with previously untreated B-CLL were included. Endpoints included overall survival (OS), overall response (OR), complete 
remissions (CR), time to progression (TTP), treatment-related morbidity and mortality. Medical databases (Cochrane Library, MEDLINE, EMBASE) and conference proceedings were searched (1990-2003) as well as internet-based registers of current clinical trials. We included full-text and abstract publications as well as unpublished data. Data extraction and quality assessment were done in duplicate. Authors of original publications were contacted to obtain missing data. Results: Out of 9 eligible studies, 5 trials with 2026 randomised pts were included, 2 trials are still ongoing, 1 trial has not ye been fully evaluated and the results of 1 trial were published including data from non-randomised patients. Compared to alkylators, treatment with purine antagonists (fludarabine, cladribine) significantly improved the relative risk for achieving an OR (RR 1.22 [95\% CI 1.13-1.30], 5 trials, $\mathrm{n}=1746$ ) and resulted in a significantly higher proportion of CRs (RR 1.93 [95\% CI 1.652.27], 5 trials, $n=1746)$. Incidence of grade III/IV infections was significantly increased in patients receiving treatment with purine antagonists (RR 1,97 [95\% 1.33-2.91]; 3 trials, $\mathrm{n}=1515$ ). There was no significant difference concerning the RR for grade III/IV neutropenia (RR 1.14 [95\% CI 0.96-1.35], 3 trials, $n=1515$ ) and therapy-related death (RR 1.05 [95\% CI 0.44-2.49], 2 trials, $\mathrm{n}=1296$ ). Incidence of hemolytic anemia was low, but significantly increased in the purine antagonist group (RR 3.18 [95\% CI 1.13-8.92], 2 trials, $n=1153$ ). Raw data from investigators concerning OS and TTP is pending. Conclusions: In patients with previously untreated B-CLL, therapy with purine antagonists results in significantly increased OR and CR rates compared to alkylator-based regimens. However, treatment with purine antagonists also augments the risk for grade III/IV infections. Results for OS and TTP will be presented at the meeting.

\section{P574}

\section{Aberrant Promoter Methylation Patterns of CDK Inhibitor, p57 ${ }^{K / P 2}$, and Cell Cycle Regulation in MCL}

Hutter G. ${ }^{1}$, Zimmermann $Y .{ }^{1}$, Scheubner M. ${ }^{1}$, Kalla J. ${ }^{2}$, Katzenberger $T^{2}{ }^{2}$, Ott G. ${ }^{2}$, Hiddemann $W^{1}{ }^{1}$, Dreyling $M .{ }^{1}$

${ }^{1}$ Dept. of Medicine III, University Hospital Grosshadern/LMU, CCG Leukemia, GSF-National Research Center for Environment and Health, Germany, ${ }^{2}$ Institute of Pathology, University Wuerzburg, Germany

Introduction: Mantle cell lymphoma (MCL) is characterized by the chromosomal translocation $\mathrm{t}(11 ; 14)(\mathrm{q} 13 ; \mathrm{q} 32)$, resulting in the overexpression of cyclin D1. However, additional genetic alterations are detectable in the vast majority of cases. The hypermethylation of $\mathrm{CpG}$ islands of the promoter region represents an alternative selective mechanism of gene inactivation. So far little is known about the differential effect and the hierarchy of promoter methylation of INK4 and $p 57^{\text {KIP }}$. Methods: MSP analysis with subsequen cloning and sequencing of PCR products were applied to determine the methylation pattern of $\mathrm{p} 57^{K I P 2}$. Results were correlated to genomic alterations in the INK4 gene cluster, $p 53$ gene inactivation and cell proliferation. RNA expression of the $557^{\text {KIP2 }}$ was investigated by RT-PCR in cell lines. Results: By analysing $p 57^{\text {IIP }}$ promoter methylation and RNA expression in differen hematological cell lines a promoter region of the gene could be identified which is functionally relevant for the expression of the gene product. The identified promoter region was methylated in 4 out of 10 tested MCL patient samples but neither in 4 control samples from healthy volunters nor in CD34+ cells. Currently, multivariate analysis of the $p 57^{K I P 2}$ gene methylation being performed in additional MCL patient samples to analyze its correlation to other genomic alterations in the INK4 gene cluster, $p 53$ gene inactivation and cell proliferation to identify the functional relevance in malignant transformation of MCL. Conclusion: Our study results proof methylation of a specific promoter region of $p 57^{K I P 2}$ is functionally relevant for gene expression. Analysis of a small number of MCL patient sample suggests that methylation of this promoter region is characteristic for MCL. Analysis of additiona patient samples is planned to identify the clinical relevance of $p 57^{K I P 2}$ methylation in MCL.

\section{P575}

Off-study Use of Alemtuzumab is as Effective as Reported in Clinical Trials in Heavily Pretreated B-CLL: A Retrospective Analysis

Fiegl M. ${ }^{1,9}$, Hopfinger G. $^{2}$, Fridrik M. ${ }^{3}$, Zojer ${ }^{4}{ }^{4}$, Walla $B{ }^{5}{ }^{5}$, Andel J. ${ }^{6}$, Schmid F.X. ${ }^{7}$, Lang A., Ludescher C. , Zabernigg A. ${ }^{8}$, Mian M. ${ }^{1}$, Büchel J., Greil R. ${ }^{10}$

${ }^{1}$ Innere Medizin/Hämato-Onkologie, Medizin-Uni Innsbruck, Austria, ${ }^{2}$ Hanusch-Krankenhaus, Wien, Austria, ${ }^{3} \mathrm{AKH}$ Linz, Austria, ${ }^{4}$ Wilhelminenspital Wien, Austria, ${ }^{5} \mathrm{KH}$ Dornbirn, Austria, ${ }^{6} \mathrm{KH}$ Steyr, Austria, ${ }^{7} \mathrm{KH}$ Bregenz, Austria, KH Feldkirch, Austria, ${ }^{8} \mathrm{KH}$ Kufstein, Austria, ${ }^{9} \mathrm{KH}$ Natters, Austria, ${ }^{10} 3$. Med. Abt., Medizin-Uni Salzburg, Austria

Purpose: Alemtuzumab (MabCampath) is the most effective monoclonal antibody in single-agent use for B-cell chronic lymphocytic leukemias (B CLL). Response rates in heavily pretreated patients range between 33 and $52 \%$. Methods: The results of a retrospective survey on the therapeutic efficacy of alemtuzumab in CLL given in a series of more than 70 patients treated in several Austrian hospitals are reported. 5\% of patients showed features of secondary PLL and Richter's transformation, respectively. Mabcampath was given alone and, in some cases, in combination with fludarabine phosphate after alemtuzumab monotherapy. After dose escalation, alemtuzumab was administered at $30 \mathrm{mg}$, three times weekly for up to 30 weeks. Results: The median age was 63 ys, and most patients were in Rai stages III and IV disease. All patients have been heavily pretreated (median 4 prior regimens, range 1-7). All patients had previously received fludarabine phosphate, and the majority was considered fludarabine phosphate-refractory. Using National Cancer Institute (NCI) Workshop criteria, objective responses to alemtuzumab in nearly $40 \%$ of pts were recorded, with CR in $10 \%$ of responders. Stable disease ( $\mathrm{SD},>2$ months) was seen in another $40 \%$, whereas about $15 \%$ of pts showed immediate progressive disease. Peripheral blood response rates were much higher, with $40 \%$ CRs and near 50\% PRs, whereas nodal responses included about 5\% CRs, and 35\% PRs. The overall response to alemtuzumab was not worse in fludarabine phosphate-refractory disease (near 40\%). Fludarabine phosphate/alemtuzumab in combination was not more potent, probably because these patients represented a unfavourable subgroup. Available survival data suggest a profound survival prolongation when compared to published series with the about 8 months' survival in fludarabine-refractory disease. CMV reactivations occurred in $10 \%$, as to be expected; interestingly, we observed two tuberculosis reactivations and one second primary tumor (Hodgkins disease), the propagation of which might have been triggered by CLL- and/or treatment-associated immunosuppression. Conclusions: Alemtuzumab is effective in these unfavourable CLL patients in terms of response and prolongation of survival. Toxicities and complications occurred in the expected range suggesting correct supportive therapies according to guidelines.

\section{P576}

BCL2 as an Independent Predictor of Survival in Diffuse Large B-Cell Lymphomas (DLBCL) - a Tissuemicroarray (TMA) Based Study

\author{
Augustin F. $1^{2}$, Pehrs A.-C. ${ }^{3}$, Zimpfer A. ${ }^{3}$, Fiegl M. ${ }^{2}$, Greil R. ${ }^{4}$, \\ Dirnhofer S. ${ }^{3}$, Tzankov A. \\ ${ }^{1}$ Institute of Pathology at the University of Innsbruck, Austria, \\ ${ }^{2}$ Department of Internal Medicine at the University of Salzburg, \\ Austria, ${ }^{3}$ Institute of Pathology at the University of Basel, Switzerland, \\ ${ }^{4}$ Department of Internal Medicine at the University of Innsbruck, \\ Austria
}

Purpose: Gene expression profiling of DLBCL revealed three distinct phenotypic variants: germinal center B-cell-like $(\mathrm{GC})$, activated B-cell-like (ABC) and a ,third“" type. ${ }^{1}$ The present study was designed to analyze the relationship between immunophenotype, clinical features and outcome in DLBCL with respect to their protein expression profile. ${ }^{2}$ Methods: A TMA comprising 113 different cases of DLBCL with complete clinical follow-up (mean observation period 40 months) was constructed. Expression of CD10, CD20, CD44, bcl-2 and bcl-6 was assessed by immunohistochemistry. Disease specific survival (DSS) was analyzed by the Kaplan-Meier method and compared by the log-rank test. Nonparametric tests were applied to demonstrate correlations between immunoprofiles and stage, IPI, LDH, $\beta 2$ - 
microglobulin, age, sex and primary site of involvement. Multivariate analysis for the effect of each expressed factor as well as for clinical parameters was performed using a Cox stepwise regression analysis. Samples were segregated into GC (bcl-2-/ bcl-6+ and/or CD10+/CD44-, n=42), ABC (bcl$2+/$ bcl-6-/CD10-/CD44, $\pm n=18$ ), type three (all other immunophenotypes, $\mathrm{n}=37$ ) and secondary transformed DLBCL (all CD44+, n=13). For statistica analysis, secondary transformed DLBCL were excluded. Results: Multistep Cox regression analysis showed IPI $(\mathrm{p}<0.0001)$, B-symptoms $(\mathrm{p}=0.012)$ and BCL-2 $(p=0.023)$ to be of independent prognostic significance for DSS in DLBCL. Multivariate analysis of all patients with an IPI score of 1 to 3, i.e. 0 to 3 risk factors, showed BCL-2 to be the only significant risk factor considering DSS ( $\mathrm{p}=0.023$, IPI: $\mathrm{p}=0.066$, Fig.1). Conclusions: In low to high intermediate risk DLBCL patients BCL-2 is the only significant risk factor considering DSS. The predictive value of BCL-2 is superior to that of IPI. This finding might be important in stratifying patients with DLBCL in regard to risk-adjusted treatment.

Fig.1: Kaplan-Meier-plot showing BCL-2-dependent survival in patients with IPI 1-3

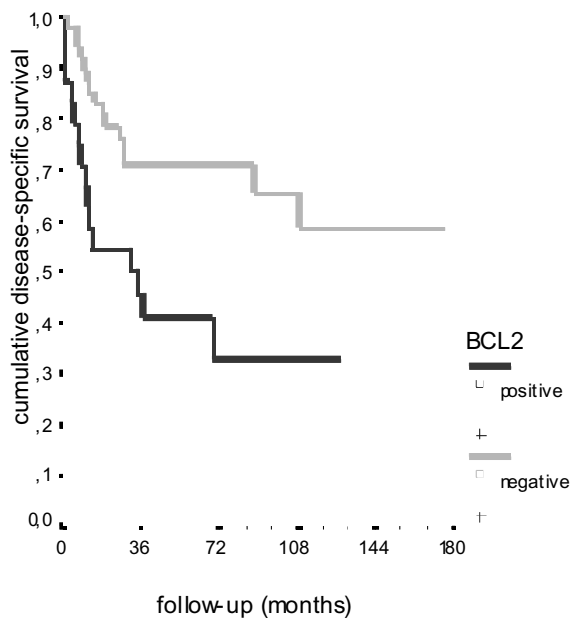

1. Alizadeh AA et al. Distinct types of diffuse large B-cell lymphoma identified by gene expression profiling. Nature 2000;403:503-11.

2. Tzankov A et al. Prognostic significance of CD44 expression in diffuse large B cell lymphoma of activated and germinal centre B cell-like types: a tissue microarray analysis of 90 cases. J Clin Pathol 2003;56:747-52.

\section{P577}

\section{Double Induction Combined with Tandem Autologous Stem Cell Transplantation in Relapsed and Refractory Aggressive Lymphomas}

Reichle A., Berand A., Holler E., Andreesen R.

Department of Hematology and Oncology, University Regensburg, Germany

Purpose: Feasibility and high efficacy of repetitive dose-intensive chemoimmuno-therapy in relapsed and refractory aggressive NHL (adjusted IPI a relapse 2 and 3 ) was proven by double-induction followed by tandem stem cell transplantation including a treosulfan-based conditioning regimen. Methods: For cytoreduction and stem cell mobilisation, 2 cycles VIPE were applied followed by two identical cycles of high-dose chemotherapy (HDCT) consisting of treosulfan $14 \mathrm{~g} / \mathrm{m}^{2}$ iv day -4 to day -2 , carboplatin 300 $\mathrm{mg} / \mathrm{m}^{2}$ iv day -4 to day -2 and etoposide $500 \mathrm{mg} / \mathrm{m}^{2}$ iv day -2 to day -4 . In B-NHLs each cycle was combined with rituximab $375 \mathrm{mg} / \mathrm{m}^{2}$. Results: So far, 26 patients (pts) mean 48 years (range 22-65), stage III: $n=7$, stage IV $\mathrm{n}=19$, have been enrolled, 5 pts with early relapse (within 6 months), 12 with refractory disease and no available matched related or unrelated donor, 7 pts with late relapse ( $\geq 6$ months). All patients with NHL received previously CHOP-based CT. Histology revealed diffuse-large cell lymphoma $(n=19)$ Hodgkins' lymphoma $(n=3)$, and pleomorphic T-cell lymphoma $(n=4)$. Only one stem cell mobilization was necessary to collect sufficient CD34+ cells for two transplantations. Median hematologic recovery ( $>1.0$ leukocytes/nl and platelets $>20 / \mathrm{nl}$ ) after $1^{\text {st }}$ and $2^{\text {nd }} \mathrm{HD}-\mathrm{CT}$ was achieved by day 10 (8-11). No therapy-related death occurred. CTC ${ }^{\circ} \mathrm{III}$ and ${ }^{\circ} \mathrm{IV}$ non-hematologic toxicities were as follows: 11 of 26 pts after $1^{\text {st }} \mathrm{HD}-\mathrm{CT}$ had ${ }^{\circ} \mathrm{III}$ toxicities (infection, vomiting, enteritis, stomatitis, diarrhea), after $2^{\text {nd }}$ HD-CT 9 of 23 pts, respectively. Complete remission ( 21 of 26 pts, $81 \%$ ) was achieved after doubleinduction $(\mathrm{n}=2), 1^{\mathrm{st}} \mathrm{HD}-\mathrm{CT}(\mathrm{n}=7)$, and $2^{\text {nd }} \mathrm{HD}-\mathrm{CT}(\mathrm{n}=12), \mathrm{PR}$ in $4 \mathrm{pts}$, and no pt had progressive disease. $\mathrm{CR}$ after double-induction and $1^{\text {st }} \mathrm{HD}-\mathrm{CT}$ was followed by continuous CR (cCR, 2 to 26 months, mean 12,5 months) in 9 of 10 cases $(90 \%)$, after $2^{\text {nd }}$ HD-CT in 6 of 12 cases $(50 \%)$. At a median observation time of 12,5 months 18 pts $(69 \%)$ are alive. Conclusions: Tandem treosulfan-based high-dose CT is feasible with manageable toxicity profile. $\mathrm{CR}$ and cCR rates argue in favor for a dose-response relationship even in high-risk patients with aggressive lymphomas. This trial has now been extended as an OSHO multicenter trial.

P578

\section{Protein Expression Analysis of Candidate Genes in} Chronic Lymphocytic Leukemia with Trisomy 12

\section{Winkler D. ${ }^{1}$, Schneider C. ${ }^{1}$, Brückle E. ${ }^{1}$, Lichter P. $^{2}$, Döhner $H^{1}$ Stilgenbauer $S$. \\ ${ }^{1}$ Department of Internal Medicine III, University of UIm, Germany, ${ }^{2}$ Deutsches Krebsforschungszentrum Heidelberg, Germany}

The pathogenic role of candidate genes involved in CLL with trisomy 12, one of the most common aberrations in CLL, is unclear. While the critical region of most aberrations in CLL could be narrowed down to a set of candidate genes, this has been difficult in the case of trisomy 12. Aim of this study was to identify specific protein expression patterns of genes located on chromosome 12 when comparing CLL cases with trisomy 12 to cases with a normal karyotype and to cell lines. 75 CLL cases (tumor load $>70 \%$ ) showing either a trisomy 12 (59 cases) or a normal karyotype (16 cases) as detected with a comprehensive FISH probe set were selected. In addition to trisomy 12 one case had a 17 p deletion, one an $11 \mathrm{q}$ deletion and 12 cases a $13 \mathrm{q}$ deletion. In the trisomy 12 group 26 cases were $\mathrm{VH}$ unmutated, in the group with a normal karyotype 11 cases. Western blotting was performed to quantify the expression of the following proteins: CKD4, CDK2, CYCLIN-D2, p27, SMAC, MDM-2, STAT6, ARF3, GLI, APAF-1, AID. Expression was compared to three lymphoma cell lines (JVM-2, EHEB, JURKAT).

The most striking differences in expression levels among the cell lines were found for CYCLIN-D2, p27, STAT6 and MDM-2. CYCLIN-D2 was not expressed by JURKAT but clearly detectable in EHEB and JVM. JURKAT showed higher expression of p27 in relation to EHEB and JVM. JURKAT cells showed a weak expression of STAT6 while EHEB and JVM demonstrated strong signals. MDM-2 detection revealed a highly specific pattern for each cell line with a lower expression of the p57 band in JURKAT as compared to JVM-2 and EHEB who showed all three MDM-2 bands (p90, p76, p57). EHEB had the highest expression of the p57 band of the three cell lines. No clear difference in the expression levels could be found between the CLL subgroups with or without trisomy 12 for the 11 investigated proteins. As expected CDK4, CDK2 and CYCLIN-D2 were generally expressed at lower levels in CLL irrespective of the trisomy 12 status when compared to the cell lines. Expression of p27 was higher in all CLL cases as compared to JVM-2 and EHEB but as strong as in JURKAT. In conclusion, when comparing CLL with or without trisomy 12 the expression of the investigated proteins was independent of the trisomy 12 status as well as of the VH mutation status. In contrast to a previous study which associated a high p27 expression with inferior outcome in CLL we were not able to detect different p27 expression levels in genetic subgroups of CLL.

\section{P579 \\ Expression Pattern of MLTIMALT1 m-RNA in B-Cell Non-Hodgkin's Lymphomas Detected by Real-Time Quantitative Polymerase Chain Reaction (PCR)}

\section{Murga Penas E.M. ${ }^{1}$, Weichel C. ${ }^{1}$, Hinz K. ${ }^{1}$, Algenstaedt $P .{ }^{2}$,} Hossfeld D.K. ${ }^{1}$, Dierlamm J.

${ }^{1}$ Department of Oncology and Hematology and ${ }^{2}$ Department of Internal Medicine; University Hospital Hamburg-Eppendorf, Germany

The MLT/MALT1 gene located on chromosome $18 \mathrm{q} 21$ was discovered due to its involvement in the translocations $t(11 ; 18)(\mathrm{q} 21 ; \mathrm{q} 21)$ and $\mathrm{t}(14 ; 18)(\mathrm{q} 32 ; \mathrm{q} 21)$, which characterize about one third of extranodal marginal zone B-cell lymphomas of the MALT-type. The $\mathrm{t}(11 ; 18)$ leads to a fusion of the apoptosis inhibitor gene API2 on 11q21 and the MLT/MALT1 gene, a 
human paracaspase. The $\mathrm{t}(14 ; 18)(\mathrm{q} 32 ; \mathrm{q} 21)$ fuses the $M L T / M A L T 1$ gene and the immunoglobulin heavy chain (IGH) locus. In addition to translocations, amplifications of MLT/MALT1 have been reported as a possible mechanism in the pathogenesis of B-cell non Hodgkin's lymphomas (NHL). Recent studies have shown that MLT/MALT1 is a crucial signaling component in the NF-kappaB activation in response to TCR induction. But the pathogenetic consequences of API2-MLT/MALT1, IGH-MLT/MALT1 and MLT/MALT1 amplifications are currently unknown.

Using a real-time PCR (LightCycler, Roche) we determined the expression of MLT/MALT1 m-RNA in a variety of B-cell NHL including follicular lymphoma (FL $\mathrm{n}=15)$, mantle cell lymphoma $(\mathrm{MCL} \mathrm{n}=9)$, extranodal MALT lymphoma (MALT $\mathrm{n}=6$ ), chronic lymphatic leukemia (CLL $\mathrm{n}=10$ ), diffuse large B-cell lymphoma (DLBCL $\mathrm{n}=14$ ), and three Burkitt lymphoma cell lines (Namalwa, CA-46, Raji). Reactive lymph nodes from 10 healthy donors were used as negative controls. $2 \mu \mathrm{g}$ of total RNA was transcribed with Superscript II reverse transcriptase and oligo (dT)-primers. Primers fo $M L T / M A L T 1$ encompassed parts of exons 16 and 17, respectively, giving rise to a product of $360 \mathrm{bp}$. MLT/MALT1 expression was normalized by using $G A P D H$ and $P B G D$ housekeeping gene products as internal references and plasmid DNA as standard for all products.

MLT/MALT1 m-RNA was found to be expressed in all 54 patients, 3 cell lines, and 10 reactive lymph nodes analyzed. The highest levels of expression were observed in the MALT lymphomas and the reactive lymph nodes. Moderate expression was found in FL, MCL, high-grade NHL, CLL, and anaplastic large B-cell lymphomas. The Burkitt lymphoma cell lines showed a low expression of MLT/MALT1. However, in all lymphoma subtypes there was a considerable interindividual variation of $M L T / M A L T 1$ expression. Similar results were obtained with $G A P D H$ and $P B G D$ normalization. We conclude that MLT/MALT1 is widely expressed in B-cell NHL, with a high expression in MALT lymphoma and that the quantitative PCR method applied in this study is an effective and reliable method for the assessment of $M L T / M A L T 1$ expression.

\section{P580}

\section{Prognostic Significance of Combined ZAP-70 and CD38 Expression Analysis in B-Cell Chronic Lymphocytic Leukemia}

\section{Schroers R. ${ }^{1}$, Griesinger F. ${ }^{1}$, Trümper L. ${ }^{1}$, von Bonin F. ${ }^{1}$,} Becker S. ${ }^{1}$, Haase D. ${ }^{1}$, Sellmann L. ${ }^{2}$, Dührsen U. ${ }^{2}$, Dürig J. ${ }^{2}$ ${ }^{1}$ Department of Hematology and Oncology, Georg-August-University Göttingen, Germany, ${ }^{2}$ Department of Hematology, University Hospital Essen, Germany

B-cell chronic lymphocytic leukemia (B-CLL) represents a heterogeneous disease with a highly variable clinical course. Previous studies have demonstrated that CD38 cell surface expression and immunoglobulin heavy-chain variable-region gene $\left(\mathrm{IgV}_{\mathrm{H}}\right)$ mutation status are important prognostic markers in B-CLL. CD38 expression is associated with unmutated $\operatorname{IgV}_{\mathrm{H}}$ genes; however, results are discordant in approximately $30 \%$ of cases. Thus, combination of $\mathrm{CD} 38$ and $\mathrm{IgV}_{\mathrm{H}}$ mutation status has been suggested to improve prediction of disease progression, response to therapy, and overall survival. Recently, expression of the protein tyrosinase kinase ZAP-70 has been identified as a prognostic marker in B-CLL correctly predicting the $\mathrm{IgV}_{\mathrm{H}}$ mutational status in $92-93 \%$ of patients. Since intracellular ZAP-70 expression can be readily determined in a flow cytometry assay, ZAP-70 analysis is now increasingly used as a convenient surrogate marker of $\mathrm{IgV}_{\mathrm{H}}$ status. Recently, we have shown that ZAP-70 and CD38 expression correlated in 63 out of 76 patients $(83 \%)$, while the remaining 13 patients $(17 \%)$ revealed discordant results, with a majority $(9 / 76 ; 12 \%)$ showing a ZAP-70+/CD38- immunophenotype. Interestingly, the ZAP-70+/CD38- patients were characterized by a longer progression-free survival than the ZAP-70+/CD38+ patients. To further investigate the prognostic significance of combined ZAP-70 and CD38 analyses, we have extended our previous study to a larger cohort of BCLL patients $(n=195)$. To this end, a discordance of CD38 and ZAP-70 expression has been revealed in 35 patients $(18 \%)$. $\mathrm{IgV}_{\mathrm{H}}$ mutational status and genomic aberrations have been determined in discordant B-CLL cases by DNA sequencing and FISH, respectively. In addition, time course analyses of ZAP-70 and CD38 expression performed in a subgroup of B-CLL patients will be presented.
P581

\section{Treatment inside vs. Outside Therapy Optimization Protocols - Methods and Progress of a Prospective Population-Based Comparison}

\section{Hoffmann W. ${ }^{1}$, Heidelbach S. ${ }^{1}$, Paulus U. ${ }^{2}$, Löffler $M{ }^{3}$}

${ }^{1}$ Institut für Community Medicine, Abt. Versorgungsepidemiologie und Community Health, Universität Greifswald, Germany,

${ }^{2}$ Koordinationszentrum für Klinische Studien, Universität Köln, Germany,

${ }^{3}$ Universität Leipzig, Institut für Med. Informatik, Statistik und

Epidemiologie, Leipzig, Germany

Background: TOPICS-ML (Clinical therapy optimisation protocols in cytostatic and radiotherapy of patients with Hodgkin's disease and high-grade lymphoma) quantitatively evaluates whether patients with Morbus Hodgkin $(\mathrm{MH})$ and high-grade non-Hodgkin's-lymphoma (NHL-h) enrolled in clinical therapy optimisation protocols (TOP) receive more often an optimum therapy than non-TOP-participants. A fair comparison requires not only valid data on inclusion and exclusion criteria for the respective TOP. Specific histology results, exact tumor stage, age of the patient, concomitant diseases, and other clinical and patient-related risk factors must be considered. Methods: TOPICS-ML is based on a population representative incidence study with active data ascertainment from multiple sources. Incident cases with $\mathrm{MH}$ (first diagnosis 1988-1998) and NHL-h (Dx 1994-1998) are examined as to their eligibility for TOP. Clinical risk factors, detailed treatment, associated side effects and complications and recurrences were abstracted actively and systematically from primary clinical documentation using a standardized instrument. Via linking these data to the information in the central TOP documentation we establish whether a certain patient was treated in a TOP or not. Ultimately, overall survival time, relapse-free survival and early and late toxicity will be compared between TOP-patients and non-participants, adjusting for individual risk factors. Present status (5/2004): Ascertainment is completed in five hospitals and active in three other hospitals. Completed records are documented for 395 patients (198 MH, 192 NHL-h, 5 with both diagnoses). Only 46 of these patients had been enrolled in TOP. For $44.6 \%$ of the non-participants TOP eligibility could be positively established. Exclusion criteria were documented for $40 \%$ of the non-participants. The remaining 52 patients are being evaluated in the study centers of the respective TOP which will decide whether or not these patients would have been enrolled based on routine algorithms. In March 2004 a comprehensive data quality analysis was performed. Results indicated relevant differences between the two groups of patients. For $50 \%$ of patients with $\mathrm{MH}$ not treated in TOP higher doses of ionising radiation were found while $19 \%$ received lower doses than in the standardised protocol. $11 \%$ of these patients did not receive chemotherapy, although they would have had they been involved in the TOP for which they qualified. From 47 patients with NHL-H who met the inclusion criteria of the specific TOPs, but were not enrolled, $18(38 \%)$ received radiotherapy which was not included in the respective TOP protocol. Future itinerary: It is planned to include 743 patients in TOPiCS-ML. Results will be instrumental to assess the potential of TOP to optimize treatment for both lymphoma entities and thus will enhance quality and equality of health care for lymphoma patients.

TOPICS-ML is funded by the BMBF (Kompetenznetz Maligne Lymphome).

\section{P582 \\ Epidemiology and Mechanism of Human B Cell Lymphoma-Associated $\mathrm{t}(14 ; 18)(I G H-B C L 2)$ and $\mathbf{t}(8 ; 14)(M Y C-I G H)$ Translocations}

\section{Schüler F. ${ }^{1}$, Hirt C. ${ }^{2,3}$, Janz S. ${ }^{3}$, Rabkin $C{ }^{2}$, Dölken G. $^{1}$}

${ }^{1}$ University Greifswald, Clinic for Internal Medicine C, Greifswald, Germany, ${ }^{2}$ Viral Epidemiology Branch, DCEG, NCl, Bethesda, USA, ${ }^{3}$ Laboratory of Genetics, NCl, Bethesda, USA

Healthy individuals harbor cell clones that contain chromosomal $\mathrm{t}(14 ; 18)(I G H-M Y C)$ and $\mathrm{t}(8 ; 14)(M Y C-I G H)$ translocations tightly associated with human B-cell lymphomas. Evidence indicates that translocation-bearing cell clones can persist for several years in peripheral blood without development of overt lymphoma. In addition, in patients in long term remission after radiotherapy for localized stage follicular lymphoma, $\mathrm{t}(14 ; 18)$-positive cells clonally related to the original tumor have been detected for up to ten years after treatment. The biology of aberrant translocation-bearing B cells in normal individuals is largely unknown. 
To gain a better understanding of the kinetics and persistence of aberrant translocation-bearing cell clones, we decided to perform an epidemiologic study on the occurrence of aberrant B cell clones in pre-morbid blood samples of individuals who later developed non-Hodgkin's B-cell lymphomas (NHL), either associated with HIV infection or not. We identified NHL patients from large prospective studies on HIV-positive individuals, using the AIDS Cancer Cohort Study (ACCS), the Multicenter Hemophilia Cohort Studies I and II (MHCS), and the NY/DC Homosexual Male Study (DCG). We also identified HIV-negative individuals from the Prostate, Lung, Colorectal and Ovarian cancer etiology study (PLCO) from which tumor tissues and pre-morbid blood specimens were available.

In a small pilot study, we examined 14 tissue sections with lymphoma from 12 patients. We were able to isolate sufficient amounts of DNA in 9 of 14 tissues. In 7 cases, we were able to determine clonotypic VDJ rearrangements using DNA sequencing. One patient showed two distinct VDJ rearrangements, possibly indicating a biclonal tumor. We then designed allele-specific oligonucleotide primers (ASO) that were combined in some cases with consensus family framework region 3 primers. Using ASO PCR primers, we were able to develop real-time quantitative PCR methods for lymphomaassociated VDJ rearrangements for four patients. This finding encouraged us that it is possible to identify the clonal VDJ rearrangement from lymphoma slides and to use this as marker for the specific detection of clonotypic cells in pre-morbid samples.

\section{P583}

\section{Treatment of Relapsed Aggressive Non-Hodgkin Lymphoma in Elderly Patients Using Rituximab and Bendamustine}

Weßendorf S. ${ }^{1}$, Basovski L. ${ }^{2}$, Viardot $A .{ }^{1}$, Schwaenen $C .{ }^{1}$, Kohlhammer $H^{1}$, Sandherr M. ${ }^{3}$, Brugger $W^{4}{ }^{4}$, Schnaitmann $R^{5}$, Pruemmer O. ${ }^{6}$, van Roye $\mathrm{C}^{7}$, Doehner $\mathrm{H}^{1}$, , Bentz M.

${ }^{1}$ Innere Medizin III, Medizinische Klinik der Universitaet Ulm, Germany, ${ }^{2}$ Tumortherapiezentrum, Klinikum Konstanz, Germany,

${ }^{3}$ II Medizinische Klinik, Zentralklinikum Augsburg, Germany,

${ }^{4}$ Innere Medizin II, Klinikum Villingen-Schwenningen, Germany,

${ }^{5}$ Medizinische Klinik I, Ostalb Klinikum Aalen, Germany,

${ }^{6}$ Innere Medizin III, Klinikum Kempten, Germany,

${ }^{7}$ Evangelisches Stift St. Martin, Koblenz, Germany

In elderly patients with relapsed aggressive non-Hodgkin lymphomas (NHL), no efficient standard therapies are available. In this study, we used a combination therapy with rituximab and bendamustine (R-Bendamustine) for patients with relapsed aggressive NHL, who were not eligible for a salvage therapy by autologous stem cell transplantation. Currently, 18 patients have been included in this study, 8 male/ 10 female, age $53-81$ years (median 74 years), number of previous therapies $1-6$ (median 1,5), 2 Patients previously received autologous stem cell transplantation. Histological diagnosis: 11 DLBCL, 4 MCL, 3 FL Grad III, stage at relapse I-IV; Patients received between 1 and 6 cycles (median 4,5) of rituximab $375 \mathrm{mg} / \mathrm{m}^{2}$ day 1 , bendamustine $100 \mathrm{mg} / \mathrm{m}^{2}$, days $2+3$ every 28 days. Therapy was tolerated well with the exception of one patient who experienced prolonged grade 4 thrombocytopenia. Currently, 13 patients can be evaluated for response. Of these seven achieved a CR and 5 patients had progressive disease. Of these 5 patients, 3 progressed under therapy and two patients had an initial response, however, progressed within two months after the completion of therapy. O the $7 \mathrm{CR}$ patients, 2 patients are still in CR after 4 and 6 months, respectively. The other 5 patients relapsed within 3 to 5 months (median 4 months). These preliminary data demonstrate that R-Bendamustine is active in aggressive lymphomas. However, remissions appear to be of limited duration in this high-risk group of patients.
P584

Prolonged Alemtuzumab (MabCampath ${ }^{\circledR}$ ) Treatment Led to a Complete Remission in Bone Marrow in a Patient with Heavily Pretreated Chronic Lymphocytic Leukemia (CLL)

\author{
Schmielau J., Graeven U., Schmiegel W., Teschendorf C.
}

Department of Medicine, Ruhr-University Bochum, Germany

The prognosis of chemotherapy refractory chronic lymphocytic leukemia (CLL) is poor with a median survival time of 10 months after conventional salvage therapies. The monoclonal anti-CD52-antibody Alemtuzumab (A, MabCampath') has shown efficacy in refractory CLL patients with response rates of 33-59\% and a median survival of 16 to 28 months. Recently, Hillmen and colleagues reported that a prolonged A treatment is able to eliminate minimal residual disease in bone marrow in about $20 \%$ of relapsed CLL patients, which correlated with a significant survival benefit (Moreton et al., Blood 2003;102(11):391b).

We present a case study of a heavily pretreated CLL patient where a complete remission was achieved after a prolonged A treatment. Diagnosis of CLL was stated in 1995. The patient (male, 63 yrs) had received 5 chemotherapy regimens including chlorambucil, fludarabine, FCM, CHOP, and bendamustine Before A treatment was started the patient was in need of platelet and erythrocyte transfusions on a regular basis due to massive bone marrow infiltration with CLL. He also had a history of several severe infections (pneumonias, herpes zoster). A was administered subcutaneously in doses of 3,10, and 30 mg during week 1 , followed by $3 \times 30 \mathrm{mg} /$ week for a total of 20 weeks. A premedication with dimetinden and paracetamol was given during dose escalation and an infection prophylaxis with pentamidine and aciclovir during treatment and thereafter. Restaging after 12 weeks of A therapy revealed a significantly improved status. The leucocyte count had dropped from 51.5 to $1.7 / \mathrm{nl}$ but due to remaining bone marrow infiltration of $45 \%$ A treatment was continued. After a total of 20 weeks of A bone marrow was completely cleared of CLL cells as detected by $\mathrm{CD} 5^{+} / \mathrm{CD} 19^{+}$flow cytometry. A treatment was very well tolerated. Major side effects were neutropenia grade 4 , which led to a treatment break of 7 days in week 4 and which resolved quickly after application of lenograstim. A grade 1 erythema during dose escalation, a grade 2 infection of upper respiratory tract, and a herpes labialis reactivation were seen. Anemia was concurrently treated with erythropoietin and no transfusion of either platelets or erythrocytes was needed beyond week 8 .

We conclude that a prolonged A treatment is a very effective treatment option for refractory CLL and is able to eliminate CLL from bone marrow. Further studies are needed to confirm the benefit of A therapy in earlier course of the disease.

\section{P585 \\ Inhibition of Protein Kinase C (PKC) Delta with Rottlerin Induces Apoptosis in B-CLL Cells and Augments Chemotherapy Induced Cytotoxicity}

\section{Ringshausen I., Weick K., Oelsner M., Peschel C., Decker T. III. Medizinische Klinik, TU München, Germany}

Purpose: We have previously shown that PKC delta is constitutively activated in B-CLL cells. In the present study, we have analyzed the mechanism of apotosis induction as well as the effets of PKC delta in the presence of survival signal and chemotherapeutic agents. Methods: The mitochondrial membrane potential, presence of active caspase 3, conformational status of Bax and apoptosis assays (Annexin V stain, Tunel assay) were performed using flow cytometric analysis. Expression of BCL-2 family proteins an $\mathrm{XIAP}$ as well as processing of caspase 8 and 9 was revealed in western blot experiments. Results: Rottlerin at $5 \mu \mathrm{M}$ (which is not toxic for normal lymphocytes) induced apoptosis in a large amount of B-CLL samples. Rottlerin was equally effective in zap70 positive- and negative samples. Rottlerin induced apoptosis was in part caspase dependent and involved processing of caspase 3, 8 and 9. Expression of antiapoptotic proteins MCL-1 and XIAP was reduced in Rottlerin treated cells and BAX conformation changed to its proapoptotic form. Rottlerin was also very effective in inhibiting survival signal like IL-4 or stromal cell contact. Treatment with Rottlerin enhanced the cytotoxicity of the chemotherapeutic agents Fludarabine, Vincristine and Daunorubicine. Conclusion: Inhibition of PKC delta might be a powerful treatment option for B-CLL given its potential to induce apoptosis, inhibit antiapoptotic survival signals and augment the cytotoxicity of chemotherapeutic agents. 
P586

First Experiences of the Use of Yttrium-90-IbritumomabTiuxetan (Zevalin ${ }^{\odot}$ ) as Salvage Therapy in Indolent Lymphoma: A Multicenter Survey

Heß G. ${ }^{1}$, Helisch A. ${ }^{1}$, Hertenstein B. ${ }^{2}$, Mezger J. ${ }^{3}$, Rendl J. ${ }^{3}$, Ruffert $K^{4}{ }^{4}$, v. Schilling $C^{5}{ }^{5}$, Hoffmann $M^{2}{ }^{2}$

${ }^{1}$ Mainz, ${ }^{2}$ Hannover, ${ }^{3}$ Karlsruhe, ${ }^{4}$ Jena, ${ }^{5}$ München, Germany

Relapsing indolent NHL is associated with a poor prognosis. The disease course is characterized by consecutive shortening of treatment success and terminally refractoriness. The introduction of $\mathrm{mAB}$ has improved results, both of first line and relapse therapies. However, even with the addition of this new tool, most patients finally die of their disease. In order to furthe improve results, radioimmunoconjugates have been developed. Yttrium-90Ibritumomab-tiuxetan (IT) $\left(\right.$ Zevalin $\left.^{\circ}\right)$, the combination of a murine CD 20 antibody and the radioisotope Yttrium-90, proved to be efficacious in phase to II clinical trials. After FDA approval 2 years ago, the drug now became available in the EU for the treatment of relapsed indolent B-NHL. Therefore, there are very limited experiences of the practical use of this new modality in Germany. Here we report on our first experiences of 10 patients treated in 5 centres outside clinical trials on an individual basis.

Patients: 10 patients with relapsed indolent NHL (FCL 9, SLL 1) up to 95 years old, median number of pretreatments 2 (range: 1-6), received a single dose of IT, for the following reasons: unavailability of other treatmen options/refractoriness, intercurrent medical problems not allowing conventional therapy, or social reasons. In selected patients dosimetry with Indium111-IT revealed median doses to BM, liver and spleen of 103, 788, $258 \mathrm{cGy}$, respectively. Radiochemical purity of IT was about $98 \%$ and mean treatment activity was $1038 \mathrm{MBq}$ (range $825-1184 \mathrm{MBq}$ ).

Importantly, no acute treatment associated side effects occurred. Main side effects during follow up were mild cytopenias mainly in weeks 4-7 after treatment. However, none of patients dropped to platelet counts below 50 and only one patient had a leukocyte nadir below $2 / \mathrm{nl}$. No transfusion support was needed, and no severe infections were noticed. Most patients accepted the treatment as comfortable and not cumbering. At this time, treatment response has been evaluated in 9/10 patients. Overall 3/9 patients achieved CR, 2/9 achieved PR, resulting in an ORR of $55 \%$. In two patients staging revealed one MR and one SD. Two patients with bulky disease showed transient responses, however thereafter progressed rapidly.

IT proved to be a safe and easy-to-perform treatment in routine clinical prac tice. Response rates have been substantial, even in heavily pre-treated patients. Data of an extended survey and follow-up will be presented during the meeting.

\section{P587}

\section{Combination of the Anti-CD20 Antibody Rituximab with} Sequential High-Dose Chemotherapy Followed by Autologous Stem Cell Support in Relapsed and Refractory Aggressive Non-Hodgkin's Lymphoma

Sieniawski M., Staak J.O., Scheuß H., Glossmann J.P., Diehl V., Engert A., Josting A.

University Hospital Cologne, Germany

Purpose: Combination chemotherapy can cure patients (pts) with NonHodgkin`s lymphoma (NHL), but those with relapse still have a poor prognosis. High-dose chemotherapy (HDCT) with autologous stem cell support (ASCT) can improve the outcome of these pts as shown in the precursor study with response rates of $46 \%(32 \% \mathrm{CR}, 14 \% \mathrm{PR})$ and FFTF/OS of $20 \% / 40 \%$ at the final evaluation (Data recently published). Chemosensitivity and achievement of minimal disease status prior to HDCT are important prognostic factors in NHL pts treated with HDCT. Rituximab demonstrated encouraging activity in aggressive NHL and showed low toxicity in the setting of combined immunochemotherapy. Methods: Eligibility criteria include pts with age 18-65 years and eligible for HDCT with histologically proven CD20+ relapsed NHL. Treatment program consists of two cycles DHAP (dexamethasone, cytarabine, cisplatin) plus rituximab $\left(375 \mathrm{mg} / \mathrm{m}^{2}\right)$; pts with PR or CR receive cyclophosphamide $\left(4 \mathrm{~g} / \mathrm{m}^{2}\right)$ plus rituximab followed by PBSC harvest; methotrexate $8 \mathrm{~g} / \mathrm{m}^{2}$ and vincristine $1,4 \mathrm{mg} / \mathrm{m}^{2}$ plus rituximab; and etoposide $2 \mathrm{~g} / \mathrm{m}^{2}$ plus rituximab. The final myeloablative course is BEAM plus rituximab followed by ASCT. Results: 20 pts (median age 57 years range 22-65) with relapsed (16 pts) and refractory (4 pts) aggressive NHL have been enrolled (stage I/II: 6, stage III/IV: 11, NA: 3). 18 pts had CHOP or CHOP-like regimens as first-line therapy, one pt. was treated with fludarabine + cyclophosphamide + alemtuzumab and one with BEACOPP. The median time to progression was 9 months. This chemoimmunotherapy combination regimen was well tolerated in all pts without side effects exceeding the toxicity expected from chemotherapy alone. 19/20 pts were available for restaging after 2 cycles DHAP with 2 CR, 12 PR, 2 SD and 3 $\mathrm{PD}$, one patient died on myocardial infarction. At the final response evaluation from 9/13 pts were in CR, 3/13 in PR and 1/13 was not avaible. Treatment was discontinued in one pt after HD-cyclophophamide due to severe heart failure. Conclusion: The preliminary results suggest feasibilty and safety of this regimen with a overall response rate of $60 \%$ (45\% complete remission and $15 \%$ partial remission); toxicity was tolerable. The combination regimen allows effective mobilization of stem cells and the tolerability of the final myeloablative BEAM was not affected by rapid sequential administration of DHAP and high doses of cyclophosphamide, methotrexate and etoposide, each in combination with rituximab.

\section{P588 \\ Expression Profiling Reveals NPM-ALK Fusion Kinase Regulated Genes and Indicates a Critical Role for Activation of AP-1 Transcription Factor}

\section{Staber B. ${ }^{1}$, Linkesch $W .{ }^{1}$, Schauer $S .{ }^{2}$, Moser $G .{ }^{2}$, Kadin M.E. ${ }^{3}$,} Dirks W. ${ }^{4}$, Hoefler G. ${ }^{2}$

${ }^{1}$ Medizinische Universitätsklinik Graz, Klinische Abteilung für Hämatologie, ${ }^{2}$ Institut für Pathologie, Medizinische Universitätsklinik Graz, ${ }^{3}$ Harvard Medical School, Boston, USA, 4DSMZ, Braunschweig

Purpose: About half of nodal anaplastic large cell lymphomas (ALCL) express the nucleophosmin-anaplastic lymphoma kinase (NPM-ALK) fusion, which is the product of the $\mathrm{t}(2 ; 5)(\mathrm{p} 23 ; \mathrm{q} 35)$ chromosomal translocation. In the present study we aimed at elucidating the consequences of NPM-ALK expression in cultured cell lines. Methods: Subtractive cDNA libraries were constructed using mRNA from 293 cells transfected with active and kinasedead NPM-ALK, as well as pools of lymphoma cell lines with and without ALK overexpression. The resulting cDNA clones were spotted on glass slides together with genes relevant for cancer pathogenesis generating cDNA microarrays comprising 4992 genes. mRNA expression patterns were analyzed in individual cell lines. Real time quantitative RT-PCR of 20 selected genes confirmed the microarray data. Results: The active NPMALK construct confers significant changes in 38 genes in 293 cells. Expression of a set of 102 genes distinguishes NPM-ALK-negative (FE-PD, MAC2A) from NPM-ALK-positive ALCL cell lines which can further be subdivided into two groups (SU-DHL-1, JB-6, SUP-M2 vs. SR-786, DEL and Karpas 299). The majority of these genes are involved in regulation of cell cycle and apoptosis. Interestingly, target genes of AP-1 transcription factor were increased in NPM-ALK expressing cell lines. Conclusions: This study reveals genes specifically regulated by NPM-ALK. Further, NPM-ALK may play a critical role in activating AP-1.

\section{P589}

\section{4-Color Flow Cytometry for Detection of Residual CLL cells and Normal B Cells after Allogeneic Transplantation}

Oelschlägel U. ${ }^{1}$, Schetelig J. ${ }^{1}$, Röllig C. ${ }^{1}$, Prange G $^{1}$, Mohm J. , Geissler G. ${ }^{2}$, Bornhäuser M. ${ }^{1}$,Ehninger G.

${ }^{1}$ University Hospital Dresden, Medical Clinic and Policlinic I, Dresden, Germany , ${ }^{2}$ Hospital Chemnitz, Medical Clinic III, Germany

Purpose: A rapid, sensitive, simple, and specific approach for MRD detection is required to evaluate the success of new therapeutic strategies in B-CLL for example allogeneic peripheral stem cell transplantation (PBSCT) including immunotherapy with Campath $1 \mathrm{H}$. B-CLL cells are immunophenotypically characterized as CD19+CD20 ${ }^{\mathrm{dim}} \mathrm{CD} 23+$ and aberrant CD5+ B cells. But after PBSCT an increased percentage of polyclonal CD5+ B cells could be present (Maloum, BJH 2002). Methods: Therefore, a more specific 4 color antigen combination including additional CD79b as marker for normal $\mathrm{B}$ cells has been designed for MRD evaluation. Results: We performed 81 flow cytometric investigations in peripheral blood or bone marrow of 14 patients with CLL before and at different intervals after PBSCT (day 14 - day 300). (1) Before transplantation the typical antigen pattern CD19+CD5+CD20 dim CD79b- was present in at least $95 \%$ of B cells in all 
patients. (2) In 34/67 follow up investigations after PBSCT all CD19+/CD5+ were negative for CD79b implicating the presence of CLL cells. This was also the case in $12 / 18$ patients with a decreased percentage $(<5 \%)$ of total $\mathrm{B}$ cells. (3) Both, CLL cells (CD79b-) and normal B cells (CD79b+) coexpressing CD19/CD5 could be detected in 10/67 measurements. (4) In further 13 samples only normal B (CD79b+) cells coexpressing CD19/CD5 were detectable. This cohort contained 4 patients with up to $46 \%$ of total B cells. The remaining 8 samples did not show any CD19+/CD5+ B cells. Conclusions: In summary, the proposed 4-color B-CLL immunophenotyping is a feasible tool for routine flow cytometry discriminating subpopulations of CD19+/CD5+ B cells. Correlation of flow cytometric results with PCR investigations and clinical course of the patients are in process.

\section{P590}

\section{Maintenance Therapy after Autologous Stem Cell Transplantation Using the Anti-CD20 Antibody Rituximab in Patients with Follicular Non-Hodgkin Lymphoma}

\section{Neumann F. ${ }^{1}$, Harmsen S. ${ }^{1}$, Martin S. ${ }^{2}$, Kronenwett R. ${ }^{1}$, Kondakci M. ${ }^{1}$, Germing U. ${ }^{1}$, Haas $R^{1}{ }^{1}$, Kobbe $G .{ }^{1}$}

${ }^{1}$ Dept. of Hematology, Oncology and Clinical Immunology, HeinrichHeine-University, Duesseldorf, Germany, ${ }^{2}$ Institute for Transuranium Elements, Karlsruhe, Germany

Purpose: Rituximab is a monoclonal antibody which binds to the CD20 antigen inducing apoptosis and complement as well as effector cell mediated lysis. CD20 is present on the malignant cells of more than $95 \%$ of patients with B-cell lymphoma. In clinical trials, treatment of B-cell non- Hodgkin lymphomas (NHL) at different stages with Rituximab in combination with cytotoxic chemotherapy or alone was shown to be efficacious. Methods: In this study, we compared a group of 11 patients with stage II-IV NHL who received Rituximab as maintenance therapy with a historical group of patients who did not receive therapy following high dose chemotherapy and autologous stem cell transplantation (PBSCT). Rituximab was given at a dose of $375 \mathrm{mg} / \mathrm{m}^{2}$ for a median number of 7 infusions (range 1-13) in time intervals ranging between 4 and 12 weeks. Molecular monitoring of $t(14 ; 18)$ was performed from samples of peripheral blood and bone marrow using nested as well as quantitative real time PCR (qPCR) based on the LightCycler technology. The median time of follow up after transplantation was 29.4 months (range 2.3-38.6) for patients who received Rituximab and 35 months (range 5.5-106) for the control group. Except for one patient in the Rituximab group all patients were in complete remission at the time of transplantation. The groups were similar with regard to age, previous therapy, disease stage and remission status at the time of transplantation. Results: Maintenance therapy was well tolerated and there was no case of opportunistic infections. The major finding is a statistically significant difference concerning the even $(\mathrm{p}=0.02)$ and the progression free survival $(\mathrm{p}=0.02)$ indicating a therapeutic advantage in the Rituximab group with a two-year event-free survival of 100 $\%$ in the Rituximab and $55 \%$ in the control group. No difference was found in the overall survival of the two groups. Two patients had PCR positive results in peripheral blood and/or bone marrow prior to Rituximab therapy and converted to negativity in PPCR as well as in nested PCR after 12 and months of Rituximab therapy. Conclusions: Maintenance therapy with Rituximab after high dose therapy and PBSCT is well tolerated and may improve event free and progression free survival in patients with follicular lymphoma.

\section{P591}

\section{The mRNA Expression of Tumor Associated Antigens (TAAs) in CLL Patients}

\section{Giannopoulos K. ${ }^{1,3}$, Greiner J. ${ }^{1}$, Rolinski J. ${ }^{3}$, Dmoszynska A. ${ }^{2}$,} Hus I. ${ }^{2}$, Döhner H. ${ }^{1}$ and Schmitt M.

${ }^{1} 3$ rd Dept. of Internal Medicine, University of UIm, Germany, ${ }^{2}$ Dept. of Haematooncology and ${ }^{3}$ Dept. of Clinical Immunology, Medical University of Lublin, Poland

Purpose: B-cell chronic lymphocyte leukemia (B-CLL) is the most common leukemia in Western countries. The disease is characterized by a long natural course, which might allow the development of immune responses against tumor cells. Moreover, most patients with B-CLL are advanced in age and therefore not eligible for agressive chemotherapy. The antileukemic effect obtained by graft vs. leukemia reaction or by donor lymphocyte infusion
(DLI) suggests the existence of immunogenic antigens in leukemias. Some antigens are shared in different types of cancers and leukemias. Identification of immunogenic leukemia/tumor associated antigens (L/TAAs) as target structures might open the way to mono- or polyvalent vaccines against leukemia including dendritic cell vaccination as well as immunotherapies using specific antibodies. Methods: We investigated the mRNA expression of eighteen L/TAAs from the literature (survivin, OFA-iLRP, BAGE, G250, MAGE1, PRAME, proteinase, Syntaxin, hTERT and WT-1), as well as L/TAAs defined previously by SEREX analysis of AML/CML patients by our group (PINCH, HSJ2, MAZ, MPP11, RHAMM and its isoforms RHAMM $^{48}$ and RHAMM- ${ }^{147}$ ) and by others (NewRen60). We examined PBMN from 30 CLL patients by conventional RT-PCR. Results: No expression of WT-1, h-TERT, BAGE, G250, MAGE1 and survivin was observed. Low $(2-20 \%)$ expression frequency of MPP11, PINCH, PRAME and proteinase was observed. We found $55 \%-90 \%$ expression frequency of RHAMM $^{-48}$, syntaxin and NewRen60 and 90-100\% of HSJ2, MAZ and OFA iLRP. Moreover the expression of OFA iLRP was very strong. We did not find any correlation between stage of disease, previous therapy and TAA mRNA expression frequency. Interstingly we found increased expression of only one RHAMM isotype - RHAMM ${ }^{48}$. RHAMM and its splice variants RHAMM $^{48}$ and RHAMM ${ }^{147}$ are not expressed on CD34+ stem cells nor in PBMN of healthy volunteers. Conclusions: RHAMM $^{-48}$ is an interesting target for future immunotherapy of CLL patients.

\section{P592 \\ How Much Rituximab is Needed to Achieve High Molecular and Clinical Remission Rates: A multicenter, Randomized Trial Comparing 1, 3 or 6 Infusions of Rituximab Added to 6 Cycles of CHOP Chemotherapy in Untreated Patients with Advanced Follicular Non-Hodgkins Lymphoma (HD2000-Trial)}

Hensel M. ${ }^{1}$, Scheuer L. ${ }^{1}$, Salwender $H_{.}{ }^{2}$, Finke J. ${ }^{3}$, Bürkle D. ${ }^{4}$, Dürk H.A. ${ }^{5}$, Käbisch A. ${ }^{6}$, Naumann R. ${ }^{7}$, Staiger H.-J. ${ }^{8}$, Kornacker M. ${ }^{1}$, Leo E. ${ }^{1}$, Fischer J. ${ }^{9}$, Ho A.D.

'Department of Internal Medicine V, University of Heidelberg, Germany, ${ }^{2}$ Allgemeines Krankenhaus Altona, Hamburg, Germany, ${ }^{3}$ Department of Internal Medicine I, University of Freiburg, Germany, ${ }^{4}$ Kreiskrankenhaus Am Plattenwald, Bad Friedrichshall, Germany, ${ }^{5}$ St. Marien-Hospital Hamm, Germany, ${ }^{6}$ Hämatologisch-Onkologische Praxis Giessen, Germany, ${ }^{7}$ Department of Internal Medicine I, University of Dresden, Germany, ${ }^{8}$ Stadtklinik Baden-Baden, Germany, ${ }^{9}$ Städtisches Klinikum Karlsruhe, Germany

Purpose: Immuno-chemotherapy with CHOP and the chimeric anti-CD20 antibody Rituximab has shown a high activity in the treatment of follicular lymphoma. Ongoing clinical trials showed an advantage in remission rates and progression free survival (PFS) or time to treatment failure (TTF) for combined CHOP or CVP chemotherapy with rituximab as compared to chemotherapy alone. However, there are no data on the frequency and dosage of rituximab required to induce the maximum effect. To evaluate how often rituximab should be added to standard chemotherapy to achieve maximum remission rates, we have initiated a prospective randomized multicenter phase II study. Methods: Patients (pts) with previously untreated stage III/IV CD20 positive follicular NHL were randomly assigned to receive 6 courses of a standard CHOP-21 chemotherapy (cyclophosphamide $750 \mathrm{mg} / \mathrm{m}^{2}$ d1; doxorubicine $50 \mathrm{mg} / \mathrm{m}^{2} \mathrm{~d} 1$; vincristine $1.4 \mathrm{mg} / \mathrm{m}^{2} \mathrm{~d} 1$; prednisone $100 \mathrm{mg} / \mathrm{m}^{2}$ $\mathrm{d} 1-5$ ), accompanied by rituximab $375 \mathrm{mg} / \mathrm{m}^{2}$ at day 0 only with the first CHOP course (arm A), with the first 3 CHOP courses (arm B) or with all 6 $\mathrm{CHOP}$ courses (Arm C). The major endpoint was the rate of molecular remission in bone marrow and peripheral blood in initially $\mathrm{t}(14 ; 18)$-positive pts, assessed by PCR. Other endpoints of the study were overall and complete response rates, toxicity rate, time to progression and time to next therapy. Results: Since September 2000, 104 patients with a median age of 57 years (range 30-79) were recruited. 33 pts were randomized to arm A, 36 to arm B and 35 to arm C. So far 45 pts have been documented completely after all 6 cycles and are evaluable for side effects. All three treatment arms were well tolerated. The overall incidence of AEs was similar in all groups. Grade 4 hematotoxicity occurred exclusively in arm b and C ( $21 \%$ in both). Gastrointestinal toxicity was more frequent in arm B and $C(p=0.03)$. There were no differences in fever and infection rates between the treatment arms. One treatment related death was observed 2 months after completion of therapy due to fulminant hepatitis $\mathrm{B}$. The overall response rate (ORR) of the whole group, which was evaluable in 56 patients so far, was $95 \%$ (53 of 56 pts), with 18 
complete and 35 partial remissions. 53 of 60 pts (88\%) showed a documented response after 3 courses. Conclusion: This multicenter, randomized, phase II trial addresses for the first time the optimal frequency and dosage of rituximab infusions in the combined immuno-chemotherapy. In the first interim analysis, there's a trend towards higher hematologic and gastrointestinal toxicity in the treatment arms with higher rituximab dosages.

\section{P593}

Primary CNS Lymphoma Treated with HD-Methotrexate, HD-Busulfan/Thiotepa, Autologous Stem Cell Transplantation and Response-Adapted Whole-Brain Radiotherapy: Preliminary Results of the OSHO-53 Study

Montemurro M., Kiefer T., Schwenke M., Al-Ali H.K., Wolf H., Herbst R., Haas A., Dölken G.

University Hospital Greifswald, Germany

Purpose: High-dose methotrexate (HD-MTX) is the single most effective agent in the therapy of primary CNS lymphoma (PCNSL). The optimal number of MTX-cycles remains to be determined, but even after multiple courses of MTX about $50 \%$ of the patients do not achieve a complete remission. Therefore, additional therapy is necessary, i.e. in most studies wholebrain radiotherapy (WBRT). But radiation grossly contributes to neurotoxicity, especially in the elderly. We wanted to investigate whether WBRT could be omitted in patients in CR after high-dose chemotherapy (HDC) with lipophilic substances, e.g. busulfan and thiotepa in combination with autologous peripheral blood stem cell transplantation (PBSCT). Methods: Ou phase II study was designed accordingly. Once diagnosis is ascertained by histology HD-MTX $\left(8 \mathrm{~g} / \mathrm{m}^{2}, 4 \mathrm{~h}, 6 \mathrm{~g} / \mathrm{m}^{2}>60 \mathrm{y}\right)$ is given on $\mathrm{d} 1$ and $\mathrm{d} 10$ followed by leucapheresis. Then patients were stratified: SD/PD receive WBRT (45Gy) reference therapy, those in CR/PR continue with HD-busulfan $(16 \mathrm{mg} / \mathrm{kg} \mathrm{BW}) /$ thiotepa $(10 \mathrm{mg} / \mathrm{kg}$ BW) (HD-Bu/TT) and PBSCT. In the case of CR treatment is finished, patients in PR receive WBRT (45gy). Time on treatment is very short (2-3mo). Results: We report the results of 19 patients at a median follow up of 9 months. Median age is 56 years (18-69), 9 females and 10 males, Karnofsky-Index 70\% (30-100). HD-MTX toxicity prevented HDC in 3 patients (1 renal tox, 1 hepatic tox, 1 embolic death). 2 patients showed NC after HD-MTX and didn't proceed to HDC. 4 of these patients underwent WBRT. The remaining 14 patients tolerated HDC with low toxicity. One patient died after HDC due to infectious complications. One patient received HDC despite SD after HD-MTX (protocol violation). Median survival of all 19 patients is 10 months (0-54mo). 10 of the 14 patients treated with HDC achieved a CR, 1 died, 2 PR, 1 PD. The latter three patients underwent WBRT resulting in an additional $2 \mathrm{CR}$ and $1 \mathrm{PR}$. In the HDC-group of patients median survival is 30months. Clinical neurotoxicity was not observed in patients receiving HDC only, but two of the three patients undergoing WBRT after HDC died of leukencephalopathy. One patient developed an abdominal and testicular lymphoma 6 month post trans plantation. After 8xR-CHOP14 this patient is in ongoing remission. Conclusions: Two cycles HD-MTX followed by HD-Bu/TT and PBSCT resulted in a $53 \%$ CR rate in patients with PCNSL. These patients showed no neurotoxicity and resumed their daily activities. Response-adapted WBRT in the other patients resulted in an overall response rate (CR/PR) of $68 \%$. These promising data have to be confirmed by further patients treated.

\section{P594}

\section{Successful Treatment of Angioimmunoblastic Lymphadenopathy with Dysproteinaemia (AILD) with Alpha-Interferon: A Case Report}

\section{Wellenhofer A., Fohr K., Abedinpour F., Nerl C.}

Städtisches Krankenhaus München Schwabing, Munich, Germany

Purpose: Angioimmunoblastic lymphadenopathy with dysproteinaemia (AILD) is recognized as a T-cell lymphoma which in most cases runs an aggressive course. The diagnosis is often difficult because of the varying clinico-pathological picture. Less then a third of the patients can be expected to have long-term remissions even after multiagent chemotherapy. We experienced a remarkable effect of interferon alpha in a case of AILD which was refractory to steroids and combination chemotherapy. Methods and Results: A 56-year old man was admitted to the intensive care unit because of high fever, pneumonia, fatigue, night sweats, lymphadenopathy and progressive weight loss. The patient presented in a very bad general condition with generalized lymphadenopathy, hepatosplenomegaly, haemolytic anaemia, thrombocytopenia, polyklonal hypergammaglobulinaemia, disseminated intravascular coagulation, skin rash and acute renal failure. The coombs' test was positive, serology revealed an acute EBV-infection.

He responded well to an immunosuppressive and cytoreductive treatment with prednisolone (100mg daily for two weeks) and cyclophosphamide (500mg). Lymph node- and bone marrow biopsy were performed and AILD was diagnosed. The patient received combination chemotherapy with CHOEP (cyclophosphamide, doxorubicine, vincristine, etoposide and prednisolone). He responded well initially but became refractory after 6 cycles of this regimen. Alpha-Interferon was started with 2 million IU/day s.c. As a result lymphadenopathy gradually disappeared, he remained afebrile and all blood values turned to normal ranges. The bone marrow (smear an trepine biopsy) showed no more infiltration by T-cell lymphoma. Maintanance therapy with 1 million IU/three times weekly is still continued. The patien did not experience any therapy-associated side effects and is able to lead a normal life. He is free from disease for more than two years. Conclusions: Alpha-Interferon is a well tolerated treatment option in patients with AILD refractory to combination chemotherapy and is able to induce long term remission as shown in this case.

\section{P595}

\section{Dendritic Cells are Significantly Reduced in Non- Hodgkin's Lymphoma and Express Less CCR7 and CD62L}

\section{Fiore F. ${ }^{1}$, von Bergwelt-Baildon M.S. ${ }^{1}$, Popov A. ${ }^{1}$, Beyer M. ${ }^{1}$, Schultze J.L.S.}

${ }^{1}$ Molecular Tumorbiology and Tumorimmunology, Clinic I for Internal Medicine, University Clinic of Cologne, Germany

Adaptive immune responses are considered to be crucial for the development and control of cancer. Various tumor entities are characterized by a specific cellular infiltrate comprising T-, B-, NK- and antigen presenting cells. A correlation between clinical outcome and tumor infiltration has been demonstrated for solid and hematologic cancers. Among these lymphoma represents an important model to understand such tumor / immune interaction since the neoplasia develops at the primary site of induction of immune responses. Dendritic cells (DC) play a pivotal role in the initiation and control of such immune responses. To address the lack of tumor control in clinically apparent non-Hodgkin lymphomas (NHL) we sought to determine, whether DC in tumor lymph nodes differ from DC in normal, reactive lymph nodes. Using flow cytometry 55 non-Hodgkin lymphomas and 33 reactive lymph nodes (RL) were analyzed for myeloid $\mathrm{DC}(\mathrm{mDC})$ and plasmacytoid DC (pDC). Overall frequency of DC in normal LN was higher than in NHL $(0.63 \%$ vs $0.15 \% ; \mathrm{p}<0.001)$ while the $\mathrm{pDC} / \mathrm{mDC}$ ratio was comparable. Interestingly, the $\mathrm{pDC} / \mathrm{mDC}$ ratio significantly differed between distinct histologies (FL, DLCL and MCL). Immunohistochemistry revealed that DC were reduced in tumor lymph nodes and distribution patterns were altered. To address, whether the reduced number of DC in NHL was due to altered homing or migratory properties we determined the expression of relevant chemokine receptors and adhesion molecules. Interestingly CCR7 and CD62L, both implicated in homing to lymph nodes, were determined to be significantly reduced in plasmacytoid DC of NHL compared to reactive lymph nodes. Correspondingly expression of CD62L was 4.2-fold lower in $\mathrm{mDC}$. To further elucidate why induction of tumor specific T-cell responses is insufficient in NHL we determined the expression of molecules relevant for antigen presentation. Interestingly, DC-SIGN and CD80 were expressed at lower frequency on $\mathrm{mDC}$ from NHL. Taken together, the number of DC in nonHodgkin lymphoma is reduced compared to normal, reactive lymph nodes and markers implicated in DC-homing and T-cell activation are expressed significantly less. These features potentially contribute to the lack of tumor control in NHL. 
P596

FC-Cam - a New, Four-Weekly Schedule with Concomitant Application of Fludarabine, Cyclophosphamide and Alemtuzumab in Patients with Relapsed/Refractory Chronic Lymphocytic Leukemia (CLL)

Elter T., Trelle S., Borchmann P., Reiser M., Schulz H., Hegener P., Schnell R., Staib P., Hallek M., Engert A.

Clinic I for Internal Medicine, University of Cologne, Germany

Purpose: Fludarabine-based combination therapies such as Fludarabine/Cyclophosphamide (FC) are the most active treatment options for CLL. New chemoimmunotherapy approaches with the addition of monoclonal antibodies have even shown more promising results. We have recently reported a very high remission rate of $86 \%$ with a new 4-weekly schedule combining Fludarabine and the anti-CD52 antibody Alemtuzumab (FluCam) in 28 heavily pretreated relapsed CLL patients (Elter et al., ASCO 2004 Abstract 2113). To evaluate if the efficacy of FluCam can be further improved we are investigating the FC-Cam regimen which combines the most potent chemotherapy FC with Alemtuzumab (A). Methods: The FCCam schedule consists of an escalation phase (Phase A) of A up to $30 \mathrm{mg}$ within 3 to 14 days, followed by the FC-Cam therapy (Phase B). F was administered at a dosage of $25 \mathrm{mg} / \mathrm{m}^{2} / \mathrm{d}(\mathrm{d} \mathrm{1-3})$ and $\mathrm{C}$ at a dosage of 200 $\mathrm{mg} / \mathrm{m}^{2} / \mathrm{d}$ (d1-3) immediately before the antibody-infusion (30 mg absolute) and repeated on day 29 for 6 cycles. Co-trimoxazole and valacyclovir were given during treatment and up to a minimum of 2 months thereafter. Results Four CLL patients (Binet C) with a median age of 56 years have been treated. All patients were heavily pretreated ( $2-4$ prior regimens, median: 3 ) and had received and responded to FluCam before FC-Cam treatmentAll four patients responded to FC-Cam (1 CR, 1 Cru, 2 PR). Side effects consisted of exanthema in 2 patients and FUO, Hepatitis (non-viral) and Arrhythmia in patient. The symptoms resolved completely. No major side effects were seen in 2 patients. Conclusions: These very preliminary data suggest that FC-Cam is feasible and highly effective in CLL and warrants further investigation. Therefore the GCLLSG has recently initiated a phase-II-study which is testing the FC-Cam regimen in less pretreated CLL patients (CLL2L-trial). To further simplify the treatment a subcutaneous administration of A has been chosen for this phase-II-study.

For further information see www.dcllsg.de. Detailed treatment data will be presented.

\section{P597}

Radioimmunotherapy with I-131-Rituximab ${ }^{\circledR}$ Partially Maintains Life Quality in Lymphoma Patients with End Stage Disease

\section{Srock S. ${ }^{1}$, Bienert M. ${ }^{2}$, Munz D.-L. ${ }^{2}$, Dörken B. ${ }^{1}$, Pezzutto A. ,} Reisinger . $^{2}$

${ }^{1}$ Medizinische Klinik m. S. Hämatologie-Onkologie, Charité

Universitätsmedizin Berlin, Germany, ${ }^{2}$ Klinik für Nuklearmedizin,

Charité, Universitätsmedizin Berlin, Germany

Introduction: At our university we have gained experience in Radioimmunotherapy using CD20 radiolabeled antibodies during the past 4 years. We have treated 10 patients with relapsed CD20 positive B-NHL who received non myeloablative treatment with I-131-Rituximab ${ }^{\circledR} .7$ of the 10 patients have been observed for a miminum of 6 months after radioimmunotherapy and are reported herein. The aim was to monitor therapeutic responses, overal survival and side effects. Methods: patients with low-grade follicular lymphoma (2), immunocytoma (1), mantle cell lymphoma (4) and diffuse large cell lymphoma (3) included in the study between 3/2000 and 11/2003 underwent dosimetric measurements $0.5-96$ hours after an preinfusion of 1.5 $\mathrm{mg} / \mathrm{Kg}$ cold antibody and infusion of $185 \mathrm{MBq}(5 \mathrm{mCi}) \mathrm{I}-131$ labelled $10 \mathrm{mg}$ Rituximab $^{\circledR}$. Total Body residence time and organ dose were calculated and entered in the MIRDOSE3 program. The maximum total body activity was 40 cGy. Results: None of our patients had significant red marrow I-131-Rituximab uptake on dosimetric measurements, the uptake being below back ground measurement. Among the 7 patients who have been observed for at least 6 months after radioimmunotherapy one patient achieved a complete remission, one patient achieved a partial remission, one patient had a disease stabilization. 4 patients had progressive disease, meanwhile 2 of them have died. As side effects, a severe drop in platelet counts occurred in mos patients between 4 and 6 weeks after therapy requiring platelet transfusions in
2 cases. There was no correlation between dosimetric estimations and hematotoxicity. Conclusions: even in severely pretreated patients radioimmunotherapy is a feasible option, that can lead to significant life prolongation with an acceptable toxicity profile. Careful patient selection is mandatory.

P598

Use of Zevalin for Lymphoma Radioimmunotherapy: Preliminary Berlin Results

\section{Srock S. ${ }^{1}$, Bienert M. ${ }^{2}$, Geworski L. ${ }^{2}$, Reisinger I. ${ }^{2}$, Dörken B. ${ }^{1}$} Munz D.-L. ${ }^{2}$, Pezzutto A.

${ }^{1}$ Medizinische Klinik m. S. Hämatologie-Onkologie, ${ }^{2}$ Klinik für Nuklearmedizin, Charité, Universitätsmedizin Berlin, Germany

Zevalin has been released 04/04 for the treatment of refractory/relapsed CD20-positive FCL after Rituximab treatment. This group of NHL includes aggressive grade III FCL as well as secondary transformed FCL. Moreover, it can be assumed that in a significant amount of DLCL a follicular background is present. At the Charité 6 pts (age 60-71 y.) have been treated so far with Zevalin. 3 pts were treated during hospitalization, 3 as outpatients. Acute toxicity was not observed but as expected cytopenias occurred 3 to 6 weeks after therapy in all pts. A heavily pretreated patient with transformed NHL needed antibiotic therapy due to neutropenic fever, none of the other pts presented with complications such as infections, bleeding, or severe anemia Responses to therapy occurred as long as several weeks after Zevalin administration, showing that delay in responses must be taken into account in pts with large tumor masses or aggressive disease. A patient with transformed, high-grade NHL who had experienced a relapse only few weeks after highdose chemotherapy showed PD for several weeks after Zevalin and required a temporary palliative treatment with weekly Dexamethason/Vindesine but eventually achieved a remarkable sustained PR 16 weeks after therapy. Altogether 3 pts achieved a PR, 2 pts achieved a CR, one patient with transformed NHL and bulky disease had a fast PD already at the timepoint of Zevalin administration and died 6 weeks after therapy.

In these pts dose-activity was measured at 1 meter distance immediately afte injection of a max. of $1,2 \mathrm{Gbq}{ }^{90} \mathrm{Y}-Z$ Zevalin. The values recorded were below $40 \mathrm{mSv} / \mathrm{h}$, which demonstrate lack of radioprotection requirements for pts and their relatives. Conclusions: accurate patient selection, time scheduling as well as good cooperation between hematology and nuclear medicine are crucial for therapy. Zevalin is well tolerated by pts, including older pts. Hospitalization is not required for radioactivity protection, the hassles for the patient are limited, frequent blood counts are mandatory between 3 and 6 weeks after therapy. Delays in responses require that evaluation of treatment efficacy should not be performed too early. A maintenance therapy such as Dexa./Vindesine appears to be feasible shortly after therapy if treatment is required until response to Zevalin occurs. Because of good feasibility and limited toxicity Zevalin should not be reserved as a "last-line" therapy to patients with large tumor burden or a fast PD.

\section{P599}

Randomized trial on the Impact of Minimal Residual Disease on Disease Free Survival in Hairy Cell Leukaemia Treated with Cladribine Alone or Cladribine Followed by Consolidation Therapy with Rituximab

\section{Strehl J.W., Mey U.J.M., Glasmacher A., Schmidt-Wolf I.G.H. Department of Internal Medicine I, University of Bonn, Germany}

Background: Hairy cell leukaemia is a rare indolent lymphoma with an incidence of about 1.5-2:1,000,000. Only patients with recurrent infection due to neutropenia, the frequent need for substitution of thrombocytes or erythrocytes, and patients with symptomatic splenomegaly have to be treated. For a long time, therapy with interferon-a has been the standard treatment, not being able to achieve durable complete remissions. Since the introduction of purine analogues cladribine and pentostatin, up to $90 \%$ of patients can achieve remission. Long term follow-up has shown that the rate of relapse in these patients is about $35 \%$ to $50 \%$ in five years. More recently published data focused on the impact of minimal residual disease on the probability of relapse: Several investigators demonstrated residual hairy cells in CR patients in $13 \%$ to $100 \%$. This wide range may partly be explained by the application of different techniques to detect hairy cells (IHC, FACS, PCR). Patients 
refractory to 1st line treatment, or relapsing after purine analogue therapy have been shown to potentially achieve durable remissions with the antiCD20-antibody rituximab; here, the tumor load before the start of immunotherapy was a critical parameter for achieving CR (Nieva et al. 2003, Thomas et al. 2004). We initiated a randomized Phase II trial to investigate the efficacy of rituximab as consolidation therapy with regard to disease free survival and MRD status. Methods: Cladribine will be administered s.c. at a dose of $0.14 \mathrm{mg} / \mathrm{kg}$ for five consecutive days. Four months later, remission and MRD status will be determined with FACS and/ or IHC. Patients with $\mathrm{PR}, \mathrm{NC}$ or PD will be treated with a salvage regimen. All patients achieving $\mathrm{CR}$ or CR-RD (i.e. $\mathrm{CR}$ with up to $5 \%$ hairy cells in the bone marrow or detectable hairy cells in the peripheral blood) will be randomized into two arms: (A) treatment with rituximab $\left(375 \mathrm{mg} / \mathrm{m}^{2}\right)$ with a loading dose (weeks $17,18,19,20$ ) and a subsequent consolidation therapy (weeks 24, 28, 32, 36); and (B) follow-up. MRD will be re-evaluated at week 40. Purpose: Primary endpoint is disease free survival of treated patients. Secondary endpoints are minimal residual disease after conventional chemotherapy and after immunotherapy with rituximab, and overall survival. Inclusion of 120 pts is planned within 48 months. Recruitment is to be started in June 2004. Patients are welcome to our facility, and other centers are invited to join our study.

\section{P600}

\section{Autologous Stem Cell Transplantation for Non Hodgkin's Lymphoma - A Single Center Report}

Kasparu H. ${ }^{1}$, König J. ${ }^{1}$, Hauser H. ${ }^{1}$, Krieger O. ${ }^{1}$, Girschikofsky M. ${ }^{1}$, Bernhart M. ${ }^{2}$, Lutz D. ${ }^{1}$

${ }^{1} 1^{\text {st }}$ Int. Department, Stem Cell Transplantation Unit, Elisabethinen Hospital, Vienna, Austria, ${ }^{2} 3^{\text {rd }}$ Medical Department, Hanusch Hospital, Linz / Vienna, Austria

Purpose: Relapse rate, disease free and overall survival as well as long term side effects after autologous stem cell transplantation (ASCT) performed between $8 / 1984$ and $9 / 2003$ were evaluated in 102 patients $(47 \mathrm{f}, 55 \mathrm{~m}$, age 15-65, median: 44,5 years) diagnosed as follicular lymphoma $(n=29)$, as aggressive B-NHL $(n=59)$, as aggressive T-NHL $(n=6)$ or as mantle cell lymphoma $(\mathrm{n}=8)$. Status at transplantation for follicular lymphoma was $\mathrm{CR} 1$ (stage III/IV): 8pts., CR 2: 8 pts. or sensitive disease (PR, CR $>2$ or responding relapse): 13 patients and for aggressive lymphoma including MCL CR 1: 25 pts., CR 2 : 16 pts., sensitive disease: 29 pts. or refractory disease: 3 patients. Results: Relapse rate for FCL was $38 \%$ within the median observation period of 40 months (1-113 mo), occuring in patients transplanted in CR 1: $12,5 \%$, in CR 2: $37,5 \%$ and in sensitive disease: $53 \%$. Overall disease free survival was $46 \%$ without reaching a plateau curve ye (CR 1 vs CR 2 vs sensitive disease: $75 \%, 36 \%$ and $31 \%$, resp.). Overall survival was $52 \%$ ( CR 1 vs CR 2 vs sensitive disease: $100 \%, 57 \%$ and 26 $\%$, resp.). Patients with aggressive lymphoma relapsed in $37 \%$ within a median observation time of 31 months (1-229 mo), occuring in patients transplanted in CR 1: $24 \%$, in CR 2: 37,5\% and in sensitive disease: $41 \%$. All 3 patients with chemotherapy refractory disease showed progression shortly after ASCT and died within 7 months. Overall disease free survival post transplant was $56 \%$ with a plateau after the last relapse at 56 months (CR 1 vs CR 2 vs sensitve disease : $72 \%, 60 \%$ and $48 \%$, resp.). Overall surviva was $66 \%$ (CR 1 vs CR 2 vs sensitive disease: $77 \%, 92 \%$ and $49 \%$ resp.) Overall survival, disease free survival and relapse rate correlates to the status of disease at time of transplantation in follicular lymphoma as well as in aggressive lymphoma. Treatment related mortality occured in 4/102 patients $(3,9 \%)$, i.e. in $3 / 42$ sensitive disease due to bacterial and/or fungal infections and in $1 / 33 \mathrm{CR} 1$ due to viral hepatits. Following late complications were diagnosed: one patient with cancer of the stomach $(d+782), 1$ patient with secondary lymphoma $(d+2719), 2$ patients with autoimmune disorders $(d+2038, d+2745), 5$ patients with hypothyresosis $(d+258-d+1248)$ and 3 patients with cataract after TBI. No secondary AML/MDS was seen so far. Conclusion: ASCT for non-Hodgkin's lymphoma with increased risk for relapse was a manageable and a beneficial treatment that led in more than half of the patients to a continuing disease free survival.
P601

\section{Rituximab Maintenance Therapy in Patients with CD20+ B-Cell Non-Hodgkin's-Lymphoma}

\author{
Witzens M. ${ }^{1}$, Hensel M. ${ }^{1}$, Schmier J.W. ${ }^{1}$, Benner A. $^{2}$, Krämer A. ${ }^{3}$, \\ Ho A.D. \\ ${ }^{1}$ Department of Hematology and Oncology, The University Hospital \\ Heidelberg, Germany, ${ }^{2}$ The German Cancer Research Center, \\ Heidelberg, Germany, ${ }^{3}$ Danish Cancer Research Institute, \\ Kopenhagen, Denmark
}

Clinical and pharmacokinetic data suggest that the effect of rituximab could be improved by prolonged exposure to the drug. To verify this hypothesis we performed a prospective randomized trial of rituximab maintenance therapy in patients with CD20+ B-cell Non-Hodgkins-Lymphoma. After completion of standard treatment patients were randomized to either observation or maintenance therapy with rituximab $\left(375 \mathrm{mg} / \mathrm{m}^{2}\right)$ every 3 months for 2 years. Patients with aggressive lymphoma were enrolled if they had achieved a complete response (CR) after initial treatment. Patients with aggressive lymphoma with residual tumor mass were examined with positrone emission tomography (PET) and qualified for randomization if PET showed no signs of tumor activity. Patients with indolent lymphoma qualified for the study if at least a partial response (PR) was achieved. Patient recruitment started in July 2002. So far 30 patients with CD20+ B-cell Non-Hodgkins-Lymphoma were enrolled in this trial. Histological subtypes included diffuse large cell lymphoma (16 pts), follicular lymphoma (7 pts), mantle cell lymphoma (5 pts), primary mediastinal lymphoma (1 pt) and marginal zone lymphoma (1 pt). No severe adverse events were observed during rituximab maintenance therapy. To date, all patients in the rituximab maintenance arm are in continuous clinical remission. In the observation arm, one patient relapsed and died. We conclude that rituximab maintenance therapy is safe and well tolerated in patients with CD20+ B-cell Non-Hodgkins-Lymphoma. Patient recruitment for this study is ongoing.

\section{P602 \\ Ifosfamide for Intraocular Lymphoma: A Clinical and Pharmacokinetic Study}

Jahnke K. ${ }^{1}$, Wagner $T^{2}{ }^{2}$, Korfel A. ${ }^{1}$, Bechrakis N. E. ${ }^{3}$, Willerding $G .^{2}$, Coupland S. E. ${ }^{4}$, Fischer L. ${ }^{1}$, Kiewe P. ${ }^{1}$, Thiel E. ${ }^{1}$

${ }^{1}$ Department of Hematology, Oncology and Transfusion Medicine, Charité-Universitätsmedizin Berlin, Campus Benjamin Franklin, Berlin, Germany, ${ }^{2}$ Medical Department I, University of Lübeck, Germany, ${ }^{3}$ Department of Ophthalmology, Charité-Universitätsmedizin Berlin, Campus Benjamin Franklin, Berlin, Germany, ${ }^{4}$ Department of General Pathology, Charité-Universitätsmedizin Berlin, Campus Benjamin Franklin, Berlin, Germany

Purpose: Intraocular lymphoma (IOL) occurs as a manifestation of a primary central nervous system lymphoma (PCNSL) or as ocular involvement of a systemic lymphoma. The prognosis is poor and the treatment has not yet been defined. The efficacy of ifosfamide (IFO) for the treatment of IOL can be assumed based on its activity against aggressive lymphomas and the ability to penetrate the CNS. Methods: We treated 5 patients with IOL: 2 with relapsed disease (patient 1 and 4), 1 with newly diagnosed IOL (patient 2), 1 with secondary IOL due to immunocytoma (patient 3 ), and 1 with PCNSL and concomitant IOL in complete remission (CR, patient 5). Patients 1 and 2 received either $400 \mathrm{mg} / \mathrm{day}$ of trofosfamide (TRO), an oral prodrug of ifosfamide, on days 1-5 followed by a 5-day drug-free interval, and $150 \mathrm{mg} /$ day, respectively. Patients 3 and 4 received either 2,000 or $1,500 \mathrm{mg} / \mathrm{m}^{2} /$ day of IFO days 1-3 over 2 hours. Patient 5 received TRO maintenance therapy with $400 \mathrm{mg} /$ day on days $1-5$, followed by a 5 -day drug-free interval. No concomitant steroids were given. Concentrations of IFO and its active 4-hydroxy (4$\mathrm{OH}$-metabolites were measured in the serum and in the aqueous humor Results: According to fundoscopy, all patients with active disease achieved CR lasting for $18,8,8+, 4$ months, accompanied by a significant increase in visual acuity. No clinically relevant therapy-related side effects were noted. Concentrations of 4-OH-metabolites in the aqueous humor were comparable to or exceeded the corresponding serum concentrations in both examined patients on IFO. In 1 of 2 examined patients on TRO, a significant concentration of 4-OH-metabolites in the aqueous humor could be detected. Conclusions: Ifosfamide can penetrate the eye and shows activity in IOL with a favorable side effect profile. 
Table: Patient characteristics. $4-\mathrm{OH}-\mathrm{IFO}_{\mathrm{aq}}$ and $\mathrm{OH}-\mathrm{IFO}_{\mathrm{s}}=$ concentration of 4-OH-metabolites in the aqueous humor and serum, respectively

\begin{tabular}{|c|c|c|c|c|c|c|c|}
\hline No. & $\begin{array}{c}\text { Age } \\
\text { (years) }\end{array}$ & $\begin{array}{c}\text { Time points of } \\
\text { measurement } \\
\text { (hours after drug } \\
\text { adminstration) }\end{array}$ & $\begin{array}{c}\text { IFO } \\
\text { concentra- } \\
\text { tion serum } \\
(\mu \mathrm{M})\end{array}$ & $\begin{array}{c}\text { 4-OH-IFO } \\
\text { concentra- } \\
\text { tion serum } \\
(\mu \mathrm{M})\end{array}$ & $\begin{array}{c}\text { IFO con- } \\
\text { centration } \\
\text { aqueous } \\
\text { humor } \\
(\mu \mathrm{M})\end{array}$ & $\begin{array}{c}\text { 4-OH-IFO } \\
\text { concentra- } \\
\text { tion } \\
\text { aqueous } \\
\text { humor } \\
(\mu \mathrm{M})\end{array}$ & $\begin{array}{c}\text { 4-OH- } \\
\text { IFOaq/4- } \\
\text { OH-IFOs } \\
\text { ratio }\end{array}$ \\
\hline 2 & 83 & 16 & - & - & 0 & 7.2 & - \\
\hline \multirow{2}{*}{3} & 77 & 0 & 2.81 & 1.56 & 4.33 & 6.6 & 4.2 \\
\cline { 3 - 9 } & \multirow{2}{*}{52} & 2 & 13.7 & 1.02 & - & - & - \\
\cline { 3 - 9 } & \multirow{2}{*}{5} & 2 & 134.41 & 2.39 & 47.41 & 1.29 & 0.54 \\
\cline { 3 - 9 } & \multirow{2}{*}{48} & 16 & 0 & 0 & - & - & - \\
\hline
\end{tabular}

\section{Poster Session: Hodgkin's Disease}

\section{$\mathrm{P} 603$}

\section{Osteonecrosis as a Complication of Treating Hodgkin's Lymphoma after BEACOPP Chemotherapy}

\section{Markova J. ${ }^{1}$, Zidka M. ${ }^{2}$, Feltl D. ${ }^{3}$, Mocikova H. ${ }^{1}$, Kozak $T .{ }^{1}$}

${ }^{1}$ Department of Clinical Haematology, ${ }^{2}$ Department of Orthopaedic Surgery, ${ }^{3}$ Department of Radiotherapy and Oncology, University

Hospital Kralovske Vinohrady, Prague, Czech Republic.

Objectives: Modern treatment schedules have produced excellent results in the management of Hodgkin's lymphoma (HL) with increasing numbers of long term survivors, but have also induced various iatrogenic effects. In this study we have analysed the incidence, risk factors, and morbidity for osteonecrosis $(\mathrm{ON})$ as a complication in patients (pts) with HL treated with BEACOPP regimens. Material and Methods: The occurence of symptomatic ON was investigated retrospectively in 124 pts with primary HL who have been treated with BEACOPP chemotherapy . 110 pts (89\%) from this group were randomised into the 3rd and 4th generation trials of German Hodgkin Lymphoma Study Group (GHSG) for intermediate and advanced stages (HD9, HD11, HD12 study) since 1995 until 2003. Median age at the time of treatment HL was 31 years ( 18 - 66). Results: During the median observation time of 38 months (r $16-99)$ ON was diagnosed in 16 pts (12.9 $\%$ ), 14 pts in advanced stages (HD9B,C, HD12) and two pts in intermediate stages (HD11C). The incidence was higher for males $8.9 \%$ (11 pts) versus females $4 \%$ (5 pts). ON was diagnosed within 3 years of starting the HL therapy, median 22 months $(\mathrm{r} 10-30)$. Multifocal ON was revealed in $9 \mathrm{pts}$ (56\%). The median cumulative prednisone dose was $8960 \mathrm{mg}$ (r 3900 - 10 000). At our institution dexamethasone was added as a component of combined antiemetic therapy, median dose was $148 \mathrm{mg}$ ( $\mathrm{r} 0$ - 280). The mean duration of chemotherapy was 5,6 months (r $3-6)$. Symptoms of pain and/or immobility were chronic in $12(75 \%)$ pts and $5(31 \%)$ out of 16 pts have undergone orthopaedic procedure (4 total hip and one knee replacement arthroplasty) and additional $3(19 \%)$ are considered candidates for surgery in the future.

\begin{tabular}{|l|c|c|}
\hline & No & \% \\
\hline $\begin{array}{l}\text { Joint (s) } \\
\text { Hip (s) only }\end{array}$ & 7 & 44 \\
\hline Knee (s) only & 4 & 25 \\
\hline Hip (s) + knee (s) & 5 & 31 \\
\hline
\end{tabular}

Table: Osteonecrosis in weight - bearing joints (patients $\mathrm{n}=16$ )

Conclusions: No correlation seems to exist between the development of ON and the total dose or duration of steroid treatment. Recent studies confirmed the importance of genetic susceptibility for the ON. Individual differences may be related to single nucleotide polymorphism in the DNA sequence of the metabolic enzyme. Dexamethasone may have additive or synergistic effects along with prednisone administered within chemotherapy and may play a leading role in the development of ON.
P604

\section{Amenorrhoea in Female Patients After Treatment for Hodgkin's Disease: A Report from the German Hodgkin Lymphoma Study Group (GHSG)}

\section{Behringer K., Breuer K., Reineke T., Sextro M., Nogovà L.,} Diehl V., Engert, A.

for the German Hodgkin Lymphoma Study Group (GHSG) University of Cologne, Cologne/Germany

Purpose: Long term survivors of successfully treated Hodgkin's disease (HD) are at risk for late complications. Among these, for female patients, infertility is of major importance affecting many young women. The subject of this analysis is to evaluate the menstrual status and to define variables having influence on amenorrhoea in young women after HD therapy. Methods: From 1994-1998, the German Hodgkin Lymphoma Study Group (GHSG) conducted one generation of clinical trials for early, intermediate and advanced stages HD (HD7-HD9) involving a total of 3186 patients. A questionnaire to document the menstrual status in young female patients after therapy was sent out by the GHSG, the presented data were updated in May 2004. Results: A total of 405 female patients $(66.6 \%)$ aged $<40$ years answered the questionnaire referring the menstrual status before and after HD therapy. For most patients $(89.6 \%)$, menstruation before the beginning of therapy was regular. After a median follow-up of 3.2 years, half of the female patients $(51.6 \%)$ receiving 8 cycles of dose-escalated BEACOPP had continuous amenorrhoea. Multivariate analysis was performed and the following factors were assessed for their influence on amenorrhoea: age, treatment, stage, and the use of oral contraceptives during chemotherapy. Amenorrhoea was significantly higher in patients receiving 8 cycles of dose-escalated BEACOPP than in patients treated with ABVD alone, COPP/ABVD or standard BEACOPP $(\mathrm{p}=0.0066)$. Moreover, amenorrhoea after therapy was higher in patients with advanced-stage HD $(\mathrm{p}<0.0001)$, in patients older than 30 years at treatment $(\mathrm{p}=0.0065)$ and in patients who did not take oral contraceptives during therapy $(\mathrm{p}=0.0002)$. Conclusions: Most female patients who are treated for advanced-stage HD experience amenorrhoea after therapy. Amenorrhoea is significantly higher in women with advanced-stage HD, in those receiving 8 cycles of dose-escalated BEACOPP, and in those older than 29 years at treatment. Furthermore, the present data suggest a possible protective effect of oral contraceptives during therapy.

P605

Lymphocyte-predominant and Classical Hodgkin's Disease: Comparison of Outcomes in three Study Generations of the German Hodgkin Study Group (GHSG)

Nogová L, Reineke T, Josting A, Behringer K, Diehl V, Engert A. University Hospital Cologne and GHSG, Germany

Purpose: The pathology and clinical presentation of Lymphocyte predominant Hodgkin's disease (LPHD) differs from other forms of Hodgkin's disease (HD), the classical type of HD (cHD) included. The European Task Force on Lymphoma project found that treatment of LPHD patients (pts) using standard HD protocol can lead to CR in more than 95\% of pts. However, survival and freedom from treatment failure (FFTF) are substantially worse in advanced stage pts compared with early stage pts. Since there are no randomized studies, the GHSG reviewed all LPHD-cases registered in the last studies and compared treatment outcome with cHD pts. Methods: We retrospectively analysed 401 LPHD pts and $8196 \mathrm{cHD}$ pts treated within the GHSG trials (HD4 to HD12). From 401 LPHD pts $43.9 \%$ were in clinical stage (CS) I, 33.2\% in CS II, $17.7 \%$ in CS III and 5.2\% in CS IV. Of the 8196 cHD pts analysed, $13.1 \%$ were in CS I, $49.9 \%$ in CS II, $23.8 \%$ in CS III and $13.2 \%$ in CS IV. Results: $87.1 \%$ LPHD pts reached CR/CRu compared to $80.7 \%$ cHD pts. $0.4 \%$ LPHD pts developed progressive disease compared to $3.4 \% \mathrm{cHD}$. The relapse rate of LPHD pts was very similar to cHD (6.9\%) $4.0 \%$ LPHD pts and $8.2 \% \mathrm{cHD}$ pts died. FFTF rates according to clinical stages in LPHD pts were following: 93\% for CS I (median observation time (MOT) 40 months), $88 \%$ for CS II (MOT 39 months), $87 \%$ for CS III (MOT 44 months) and 70\% for CS IV (MOT 43). Appropriate OS rates were: 99\% for CS I, 95\% for CS II, 95\% for CS III and 79\% for CS IV. Full analysis of data will be presented. Conclusions: cHD pts present more frequently with advanced stages compared with LPHD pts. FFTF and OS rates for LPHD pts showed significant differences between early and advanced stages. Comparing LPHD and cHD pts we found differences in treatment outcome in 
respect of $\mathrm{CR} / \mathrm{CRu}$, progressive disease or mortality. Surprisingly, there were no differences in terms of relapses. In contrast to previous reports, our data suggest that relapses in LPHD and cHD pts seem to be comparable and not more frequent.

\section{P606}

\section{Gender-Specific Aspects and Factors Influencing the Treatment and Outcome of Males and Females with Hodgkin's Lymphoma}

Klimm B., Reineke T., Haverkamp H., Eich H.T., Josting A., Pfistner B., Diehl V., Engert A.

for the German Hodgkin Lymphoma Study Group (GHSG), University Hospital Cologne, Germany

Purpose: Male gender has been identified as adverse prognostic factor in patients with advanced-stage Hodgkin's lymphoma (HL). The purpose of this analysis is to investigate sex differences regarding pre-treatment and therapyrelated variables. The further aim is to analyze the influence of these genderspecific factors on the outcome of males and females in all stages of Hodgkin's lymphoma. Methods: Between 1988 and 1998 the GSHG conducted two trial generations for early, intermediate and advanced HL (HD4 - HD9). The present analysis comprises 4626 patients of all stages, aged 15 to 75 years who were enrolled into these multicenter studies an registered in the GHSG database. Patients were treated according to the GHSG-study protocols. Results: 2050 female and 2576 male patients were suitable for this retrospective comparison. The median observation time was 5 years (HD7-9) and 7 years (HD4-6), respectively. Patients in each group had comparable profiles in terms of age, performance status, stage, histolog ical subtype, clinical risk factors and prognostic factors of the Internationa Prognostic Score. There were slightly more nodular sclerosis subtypes and mediastinal masses in women, and a few more mixed cellularity subtypes in men. Acute toxicities from chemotherapy were distributed almost equally except for hematotoxicity: females showed higher rates and grades of anemia and in particular, leucopenia. Disease response to treatment was similar in both groups: $90.7 \%$ of females (versus $89.7 \%$ of males) reached a complete remission, $2.0 \%$ (versus $2.8 \%$ ) a partial remission, $0.4 \%$ (versus $0.2 \%$ ) had no change, and $5.0 \%$ (versus $5.3 \%$ ) had progressive disease. However, a lower rate of relapse $(9.1 \%$ vs $11.8 \%)$ and death $(10.3 \%$ vs $14.6 \%)$ was observed in females. Univariate analysis revealed significant better rates in terms of FFTF and OS for females. For multivariate analyses the following variables were included: sex, age, stage of disease, B-symptoms, mediastinal mass, and leucopenia grade III/IV. Sex was not identified as an independent prognostic factor in terms of FFTF ( $\mathrm{p}=0.1665)$, however lower stages of disease $(\mathrm{p}<.0001)$, less B-symptoms $(\mathrm{p}=0.0002)$, younger age $(\mathrm{p}<.0001)$, and leucopenia grade III/IV ( $\mathrm{p}=0.0001)$ were significant variables to which bette outcome in females can be related. Particularly, leucopenia grade III/IV has a great impact on better FFTF in females: when eliminating the factor leucopenia from the model, an almost significant p-value can be observed for gender $(p=0.0620)$. Conclusions: In this large retrospective analysis of the GHSG database, female patients had better FFTF and OS compared to male patients. The protective role of severe leucopenia in females supports the rationale for a more individualized, response-adapted therapy.

\section{$\mathrm{P} 607$}

The CC Thymus and Activation Related Chemokine (TARC) Is a Prognostic Factor in Primary Hodgkin's Disease

\section{Weihrauch M.R. ${ }^{1,2}$, Manzke O. ${ }^{3}$, Diehl V', Bohlen H. $^{3}$, Wolf J., ${ }^{1,2}$, Schultze J.L. ${ }^{1,2}$}

${ }^{1}$ Molecular Tumor Biology and Tumor Immunology, University of Cologne, Germany, ${ }^{2}$ Clinic I for Internal Medicine, Hematology and Oncology, University of Cologne, Germany, ${ }^{3}$ ATABIS GmbH, Cologne, Germany

Purpose: The CC thymus and activation related chemokine (TARC) is a protein, which is highly expressed by Reed-Sternberg cells in Hodgkin's disease (HD) and is found in the majority of HD patients. Methods: Within several trials conducted by the German Hodgkin-study group 63 HD patients were elected based on clinical response to study serum TARC levels by ELISA. TARC levels from 34 patients with continuous complete response (CCR), 15 patients with relapse, and 14 patients with progressive disease (PD) were correlated with freedom from treatment failure (FFTF) and survival. Results: As defined in healthy donors (mean value $+2 \mathrm{x}$ standard deviation), a TARC level greater than $500 \mathrm{pg} / \mathrm{ml}$ was considered as elevated. The mean TARC level of all patients at baseline and after completed primary treatment were $10,688 \pm 14,719 \mathrm{pg} / \mathrm{ml}$ and $1,868 \pm 4,512 \mathrm{pg} / \mathrm{ml}$, respectively. Baseline and post-treatment TARC levels of patients with PD were significantly higher than those of patients with $\mathrm{CCR}(\mathrm{p}=0.0005$ and $\mathrm{p}=0.002)$ and of patients with relapse $(\mathrm{p}=0.026$ and $\mathrm{p}=0.025)$. While pre-treatment levels of TARC correlated significantly with FFTF $(\mathrm{p}=0.0003$ ) but not survival, posttreatment levels correlated significantly with FFTF $(\mathrm{p}=0.0003)$ and survival $(\mathrm{p}=0.0001)$. A TARC level greater than $2000 \mathrm{pg} / \mathrm{ml}$ after completed treatment was a significant risk-factor for poorer survival $(\mathrm{p}=0.020)$, but not for relapse. Conclusions: Monitoring serum TARC levels in HD patients adds valuable information about therapy success in HD patients, especially those with PD and should therefore be further evaluated in future trials.

\section{P608}

Elderly Patients with Intermediate Stage Hodgkin's Disease (HD) have a Poorer Outcome Compared to Younger Patients Particularly when Treated with Combined Modality Treatment and Extended Field Radiotherapy (EF)

Engert A., Haverkamp H., Eich H., Josting A., Pfistner B., Diehl V. Department of Internal Medicine I, University Hospital of Cologne, Germany

Purpose: The HD8 study of the German Hodgkin Study Group (GHSG) demonstrated that involved field (IF) radiotherapy is equally effective when compared with EF radiotherapy after four cycles of chemotherapy $(2 \mathrm{x}$ COPP/ABVD). Since there are indications that elderly patients with HD might fare worse depending on the type of treatment applied, we revisited the HD8 data for possible differences between younger and older patients. Methods and Results: A total of 1204 patients were randomised to receive two double cycles of COPP/ABVD and either $30 \mathrm{~Gy} \mathrm{EF}+10 \mathrm{~Gy}$ bulk or 30 Gy IF + 10 Gy bulk. Of these, 98 evaluable patients were older than 60 years and 1038 patients were younger than 60 years. In general, there were more risk factors such as B-symptoms, elevated ESR, and poorer Karnofski index in the elderly group. On the other hand, there were fewer bulky tumours, large mediastinal tumours and a lower number of lymph node areas involved in elderly patients. The toxicity of treatment was more pronounced in elderly patients with 76 of 96 patients experiencing chemotoxicity Grade III or IV (79\%) compared with 699 of $1018(69 \%)$ in those younger than 60 years. After a median follow up of 52 months, the 5-year-FFTF was $85 \%$ in younger patients and $63 \%$ in patients older than 60 years $(\mathrm{p}<0.001)$. The 5 -yearoverall survival was $94 \%$ for patients younger than 60 years and $66 \%$ for patients older than 60 years $(\mathrm{p}<0.001)$. In addition, patients older than 60 years treated with EF had a trend for worse FFTF and overall survival compared to those receiving IF radiotherapy. Conclusions: Event-free and overall survival of patients older than 60 years old are worse compared with younger patients. In particular, patients older than 60 years receiving EF radiotherapy had a poorer prognosis

\section{P609}

\section{Phase I/II Study of a Fully Human Anti-CD30 Monoclonal Antibody (MDX-060) in CD30 positive Lymphoma}

\section{Borchmann P., Schnell R., Graziano R., Yellin M., Engert A University Hospital Köln, Germany}

Purpose: CD30 is constitutively expressed on Reed-Sternberg cells in HD and on ALCL cells and represents an attractive target for monoclonal antibody (mAb) therapy. MDX-060 is a fully human $\operatorname{IgG} 1 \kappa \mathrm{mAb}$ that recognizes CD30 with nanomolar affinity and mediates killing of HD and ALCL cell lines in vitro and in xenograft tumor models. MDX-060 cross-reacts with activated $\mathrm{T}$ cells from cynomologous monkeys; in preclinical studies high doses of MDX-060 (30 mg/kg x 3) were well tolerated, with no drug-related clinical or pathological findings. Methods: A Phase I/II open-label, doseescalation study of MDX-060 in patients with relapsed or refractory HD, ALCL, or other CD30+ lymphomas (without curative options) has been initiated to characterize the safety and pharmacokinetic profile of the drug and to determine preliminary evidence of activity. In the Phase I portion, which has been completely enrolled, MDX-060 was administered intravenously at dose levels of $0.1,1,5$, or $10 \mathrm{mg} / \mathrm{kg}$ weekly for 4 weeks to cohorts of 3-6 patients. In the Phase II portion, expanded cohorts have received MDX-060 at 10 or 15 
$\mathrm{mg} / \mathrm{kg}$. All patients are to be assessed for toxicity; assessment of response is to be made at Month 2 of the study. Results: To date, 21 patients $(\mathrm{HD}=16$, ALCL=3, Other=2) have been treated. MDX-060 has been well tolerated and no maximum tolerated dose has been identified. There have been no significant infusion-related reactions and no opportunistic infections have occurred. There has been 1 episode of possible drug-related toxicity in the $1 \mathrm{mg} / \mathrm{kg}$ dosing cohort. This patient, who had a history of chronic GVHD, developed elevated (Grade 3) liver transaminase levels. While efficacy assessments have not yet been completed in all patients, 1 patient with ALCL in the $1 \mathrm{mg} / \mathrm{kg}$ cohort had a complete response to therapy of 4 months duration and 1 patient with Hodgkin's disease showed a CR in the $15 \mathrm{mg} / \mathrm{kg}$ cohort. Plasma concentrations of MDX-060 were determined using a CD30 binding assay. In the 0.1 $\mathrm{mg} / \mathrm{kg}$ cohort, MDX-060 concentrations were below the quantitative limit of the assay. In the 1 and $5 \mathrm{mg} / \mathrm{kg}$ cohorts, MDX-060 trough and peak levels ranged from approximately $4-27 \mu \mathrm{g} / \mathrm{mL}$ and $28-140 \mu \mathrm{g} / \mathrm{mL}$, respectively. The half-life has not yet been determined. No patients tested thus far have demonstrated antibody responses to MDX-060. Conclusions: In conclusion, preliminary evidence indicates MDX-060 to be well tolerated, with minimal toxicity. In addition, we have seen clinical activity with complete responses observed in 2 patients so far. The study continues to accrue patients and response assessment is ongoing. Updated data will be presented.

\section{P610}

\section{Fertility in Male Patients with Hodgkin's Disease after Therapy - Results from the German Hodgkin Lymphoma Study Group (GHSG)}

\section{Sieniawski M., Josting A., Breuer K., Diehl V., Engert A. University Hospital Cologne, Germany}

Purpose: Treatment results in Hodgkin Disease (HD) have improved tremendously over the last two decades. Therefore long term sides effects of therapy are of growing importance. Infertility after therapy of HD is considered as a side effect of chemotherapy and radiotherapy. However patients with HD have increased risk for inadequate semen quality even prior to treatment. To investigate the influence of therapy on the fertility status in patients with HD we performed semen analysis before and after treatment. Methods: Semen quality were evaluated in patients with first diagnosis of HD enrolled into trials of the GHSG between 1988 and 2002, in 40 centers in Europe. Patients had no history of chemotherapy or radiotherapy. All semen analysis were evaluated according to WHO-guidelines. Results: We included 111 male patients with a median age of 26 years (range 16-52 years). At first diagnosis 10 patients were in clinical stage (CS) I, 60 in CS II, 60 in CS III and 8 in CS IV; systemic symptoms were present in 52 patients. In 9 patients therapy regimens consisted only of chemotherapy, in 12 of radiotherapy and in 90 of combined modality. 71 patients underwent fertility screening before therapy; normospermia was diagnosed by 19 patients, other 52 had inadequate semen quality. All 111 patients underwent at last once a fertility screening afte therapy; in 38 patients $(34 \%)$ a recovery of spermatogenesis was observed. Table 1 shows time of onset of spermatogenesis after the end of therapy.

\begin{tabular}{|l|l|l|l|l|}
\hline Time & $1^{\text {st }}$ year & $2^{\text {nd }}$ year & $3^{\text {rd }}$ year & after $3^{\text {rd }}$ year \\
\hline Patients & 7 & 9 & 10 & 12 \\
$38(100 \%)$ & $18 \%$ & $24 \%$ & $26 \%$ & $32 \%$ \\
\hline
\end{tabular}

From 12 patients treated with radiotherapy $11(92 \%)$ recovered from azoospermia, from 9 patients treated only with chemotherapy only 1 patien recovered and from 90 patient treated with chemotherapy and radiotherapy recovered $26(29 \%)$. Recover rate from azoospermia was lower in patients treated with BEACOPP chemotherapy, with systemic symptoms and elevated BSG. Conclusions: We confirmed that HD patients had inadequate semen quality even prior to treatment. The majority of patients had azoospermia after treatment, but recovery of spermatogenesis was observed, in general after 2 years after the end of therapy. Patient treated only with radiotherapy recovered in higher frequnency. Further studies on this area are urgently warranted.
P611

Identification of Target Genes of Tyrphostin AG17 in Treated Hodgkin and Reed Sternberg Cells by Gene Expression Profiling

Kussebi N. ${ }^{1}$, Schoof N. ${ }^{1}$, Landgrebe $J^{2}$, Stürzenhofecker B. ${ }^{1}$, Brunner E. ${ }^{3}$, Trümper L. ${ }^{1}$, Kube $D .{ }^{1}$

${ }^{1}$ Abteilung Hämatologie und Onkologie, ${ }^{2}$ Abteilung für Biochemie, ${ }^{3}$ Medizinische Statistik, Göttingen, Germany

Constitutive activation of signal transducer and activator of transcription 3 (STAT3) is observed in a variety of hematopoietic and solid tumors. Classica Hodgkin Lymphoma (cHL) is a malignancy of unknown pathogenesis. The malignant Hodgkin and Reed/Sternberg cells derive mainly from germinal center B cells but have a heterogeneous and largely uncharacterized phenotype and thus make it difficult to design targeted drugs. Tyrphostins are protein kinase inhibitors and were proven to be potent inhibitors of cell proliferation and cell survival of different tumor entities including cHL. Recently we showed that treatment of cHL cells with tyrphostin AG17 went along with decreased levels of constitutive STAT3 phosphorylation as well as reduced DNA-binding. For a comprehensive description of AG17 action in cHL a gene expression profile analysis using Micro-cDNA-arrays containing 32.000 genes was performed. 143 transcripts were found to be affected of which 70 show a downregulated and 73 an increased expression. The remaining transcripts are of so far unknown function (expressed sequence tag/EST). A selected number of genes were analysed by Taqman-Realtime-PCR.

The identified AG17 affected genes may play an important role in the pathogenesis of $\mathrm{cHL}$ and may be considered for therapeutic targeting in tumors with deregulated STAT3 signalling.

\section{P612}

Sequential Application of Chemotherapy and Monoclonal CD 20 Antibody: Successful Treatment of Advanced Composite-Lymphoma

\section{Linck D., Lentini G., Tiemann M., Kiehl M.G., Fauser A.A.} Parwaresch R., Basara N.

Clinic for BMT and Hematology/Oncology, Idar-Oberstein and Institute for Hematopathology and lymph node registry Kiel,

University of Kiel, Germany

We describe successful treatment of a 38-year old patient with composite lymphoma stage $\mathrm{IV}_{\mathrm{A}}$, who presented with multifocal enlarged lymph nodes. The lymph node histology showed classic morphometric features of Hodgkin's lymphoma, mixed cellularity subtype and follicular B-cell lymphoma. Immunophenotypic analysis showed immunoreactivity for CD20, CD10 and Ki-67 in the malignant small cell population. The areas of Hodgkin's lymphoma demonstrated positive immunoreactivity for CD30 and CD20 in the Hodgkins's cells. Both cell populations were bcl2-oncoprotein positive. Eight courses of dose-escalated BEACOPP (Cyclophosphamide $1250 \mathrm{mg} / \mathrm{sqm}$ d1, Doxorubicine $35 \mathrm{mg} / \mathrm{sqm}$ d1, Etoposide $200 \mathrm{mg} / \mathrm{sqm}$ d 1 to 3 , Bleomycine $10 \mathrm{mg} / \mathrm{sqm}$ and Vincristine $2 \mathrm{mg}$ d8) were administered. Staging after chemotherapy showed a partial remission radiologically. Histology confirmed persisting folicular B-cell lymphoma without bone marrow infiltration and no evidence of Hodgkin's lymphoma. Mobilisation of peripheral hematopoietic stem cells failed, therefore an innovative therapeutic option was needed in order to obtain the most favourable results in this patient. $\mathrm{He}$ was treated with monoclonal CD 20-antibody (Rituximab) $10 \mathrm{mg} / \mathrm{kg}$ bw weekly for eight consecutive weeks due to a marked positivity of CD 20antigen in follicular lymphoma cells. This procedure was well tolerated and final staging showed a complete remission of the composite lymphoma namely Hodgkin's lymphoma and marginal B-cell lymphoma. This patient is in CR 28 months after treatment. In conclusion, in the rare event of a composite lymphoma the combination of chemotherapy and subsequent immunotherapy might be considered as a promising therapeutic option to achieve a long lasting remission. 


\section{Poster Session: Lung Cancer}

P613

\section{Positive Endothelial Progenitor Cells Contribute to the Tumor Vasculature in Non-Small Cell Lung Cancer}

Hilbe W. ${ }^{1}$, Dirnhofer . $^{2}$, Oberwasserlechner F. ${ }^{1}$, Schmid $T^{3}$, Gunsilius E. ${ }^{1}$, Hilbe G. ${ }^{4}$, Wöll E. ${ }^{1}$, Kähler C.M.

${ }^{1}$ Department of Internal Medicine, University Hospital, Innsbruck, Austria, ${ }^{2}$ Institute of Pathology, University of Basel, Switzerland,

${ }^{3}$ Department of Surgery, University Hospital, Innsbruck, Austria

${ }^{4}$ Department of Surgery, Natters State Hospital, Austria

Purpose: Recent results generated in a mouse model postulate that tumor angiogenesis/vasculogenesis can be initiated and maintained by bone marrow-derived endothelial progenitor cells. The present study investigated the distribution and frequency of CD133-positive endothelial progenitor cells in non-small cell lung cancer (NSCLC) patients (tumor tissue and tumor-free lung regions) and healthy controls using fresh-frozen specimens. The novel marker CD133 identifies human hematopoetic precursor cells as well as human endothelial progenitor cells. Methods: 79 lung cancer specimens and 66 adjacent histologically tumor-free tissues of the same patient cohort were analyzed; 11 autopsy specimens from control patients who did not suffer from any malignant disease served as control. Cryostat sections were stained against CD133, CD31, VEGFR-2 (KDR), p53 and the proliferation marke Ki-67 and correlations analysed. Results: 43/63 (68\%) valuables tumor specimens revealed increased numbers of CD133 positive cells and in some cases capillary forming CD133 positive structures were detectable. Additionally, $48 \%$ showed elevated expression of KDR and 46\% increased MVD. Increased CD133 expression marginally correlated with elevated KDR expression but not with p53 and Ki-67. Conclusions: A significant increase in CD133 positive cells was documented in NSCLC patients suggesting an involvement of endothelial progenitor cells in tumor vasculogenesis and tumor growth in NSCLC patients.

\section{P614}

\section{Low grade MammaglobinB mRNA Expression in Non-Small Cell Lung Cancer}

Hilbe W. ${ }^{1}$, Haun M. ${ }^{1}$, Dlaska M. ${ }^{1}$, Fiegl M. ${ }^{1}$, Dirnhofer S. ${ }^{2}$, Duba H.C. ${ }^{3}$, Schmid T. ${ }^{4}$, Wöll E. ${ }^{1}$, Grünewald K. ${ }^{1}$

'Department of Internal Medicine, Innsbruck University Hospital, Austria, ${ }^{2}$ Institute of Pathology, University of Basel, Switzerland, ${ }^{3}$ Department of Medical Biology and Human Genetics, University of Innsbruck, Austria, ${ }^{4}$ Department of Surgery, Innsbruck University Hospital, Austria

Purpose: Recently, our group reported that mammaglobin B (hMAM-B) is regularly expressed in lung cancer, as detected by reverse-transcriptase polymerase chain reaction (RT-PCR). The present study investigated frequency and levels of hMAM-B expression in an extended cohort of NSCLC patient and correlated hMAM-B expression with clinical and immunohistochemica parameters. Methods: 73 native, fresh-frozen tumour-infiltrated lung cance specimens and 58 adjacent, histologically tumour-free specimens were analysed. Furthermore, 10 lung specimens from patients who died of diseases other than cancer served as controls. Cell suspensions of all specimens were quantitatively evaluated by RT-PCR for expression of hMAM-B. Additionally, corresponding sections were stained using monoclonal antibodies labelling EGFR, erbB-2, Ki-67, p53 and CD31. Finally, the occurrence of loss of heterocygosity represented by monosomy for chromosomes 7, 9 and 17 was evaluated by fluorescence in-situ hybridisation (FISH). Results: In tumours, the frequency of hMAM-B expression was comparable to that in corresponding tumour-free and control specimens $(53 \%, 41 \%$ and $40 \%$, respectively), whereas the level of hMAM-B expression was significantly reduced in tumours. Interestingly, tumour-free and possibly premalignan tissue adjacent to the primary tumours showed the same level of hMAM-B expression as the tumour-free cohort, suggesting that loss of hMAM-B expression is a phenomenon occurring late in lung carcinogenesis. hMAM-B expression correlated significantly with reduced microvessel density within the NSCLC specimens and reduced proliferative activity defined by ki-67 expression, indicating that hMAM-B expression is associated with a more favourable subtype of NSCLC. hMAM-B positive cases harboured an increased rate of LOH of the chromosomes 9 and 17. Conclusion: hMAM-B expression is progressively lost in NSCLC evolution. Detection of hMAM-B expression potentially identifies NSCLC patients with a prognostically favourable course of disease; however, inconsistency of hMAM-B expression in NSCLC suggests that hMAM-B is not useful as marker for metastatic cell detection in staging procedures.

P615

\section{Efficient in Vitro Expression of Human Reverse Transscriptase (hTERT) in Dendritic Cells of Lung Cancer Patients}

\author{
Brill A.-K. ${ }^{1}$, Klapper W. ${ }^{2}$, Weingartz U. ${ }^{3}$, Bolz I. ${ }^{1}$, Heiser A. ${ }^{3}$, \\ Kabelitz D. ${ }^{3}$, Kneba M. ${ }^{1}$, Gahn B. \\ ${ }^{1}$ Department of Internal Medicine II, ${ }^{2}$ Department of Pathology, \\ ${ }^{3}$ Department of Immunology, University Hospital Schleswig-Holstein \\ Campus Kiel, Germany
}

Purpose: Patients with advanced lung cancer have a poor prognosis after standard radiochemotherapy. An innovative treatment strategy is the targeting of tumor associated antigens with cellular effector cells. The telomerase catalytic subunit (hTERT) is an attractive target for cytotoxic T cells that is highly expressed in both NSCLC and SCLC cells. The aim of the study was the expression of hTERT in dendritic cells (DC) that are the most powerful antigen presenting cells. Methods: We used electrotransfection of DCs with hTERT mRNA that enables an HLA independent whole antigen approach potentially targeting a wide range of hTERT epitopes. Immature, i.e. nonproliferating human DCs were prepared from peripheral blood monocytes in serum-free growth medium, GM-CSF and IL-4. Subsequently the DCs were electroporated in transfection buffer with mRNA and matured in a cytokine cocktail consisting of IL-1beta, IL-6, TNF-alpha and PGE2. Results: To optimize mRNA electroporation conditions the DCs of a healthy individual were transfected with green fluorescent protein (GFP) mRNA.The percentage of electrotransfected DCs was $31 \%$ determined by selecting GFP expressing cells using flow cytometry. Importantly the RNA electrotransfected DCs retained their typical morphological and immunophenotypical characteristics, expressing high levels of HLA-DR and no lineage markers. CD83 as an indicator of maturation was expressed in $42 \%$. The costimulatory molecules CD80 and CD86 were expressed in 73\% and 97\%. Having established electroporation parameters we transfected monocyte derived DCs with hTERT mRNA in 2 lung cancer patients. Verification of transfection efficiency was performed by analyzing the induction of telomerase activity with the TRAP assay. 24 hrs after electrotransfection the measured activity in the DCs was equivalent to HL60 cells that biologically express high levels of hTERT. Non-transfected DCs did not show any telomerase activity. Conclusions: These data show that strong hTERT expression can be achieved in DCs of lung cancer patients using mRNA electrotransfection. They provide the basis for further preclinical and clinical studies.

\section{P616 \\ Expression of Fragile Histidine Triad (FHIT) Protein is Correlated with Prognosis in Patients with Small Cell Lung Cancer}

Rohr U.-P. ${ }^{1 *}$, Rehfeld N. ${ }^{1}$, Geddert $H_{.}{ }^{2}$, Pflugfelder L. ${ }^{1}$, Steidl U. ${ }^{1}$, Fenk R. ${ }^{1}$, Kronenwett $R .{ }^{1}$, Haas $R$.

${ }^{1}$ Klinik für Hämatologie, Onkologie und klinische Immunologie, University of Düsseldorf, Germany, ${ }^{2}$ Pathologisches Institut, University of Düsseldorf, Germany

The fragile histidine triad (FHIT) protein, a putative tumor suppressor in humans, is frequently lost in human lung cancer. So far, there are no data available on prognostic impact of FHIT protein expression in small cell lung cancers (SCLC). In this report, 225 patients with SCLC were retrospectively analyzed for FHIT protein expression and prognosis using formalin-fixed, paraffin-embedded tissue sections. As assessed by immunohistochemistry, FHIT protein expression was observed in $61.8 \%$ of the SCLC tumors. The Kaplan-Meyer survival analysis with log-rank and Chi-Square tests was performed in order to identify prognostic factors for better overall survival. A lack of FHIT protein was significantly associated with poor survival with a median survival of $157 \pm 18$ days compared to $210 \pm 18$ days for those patients with FHIT protein positive tumors $(\mathrm{p}=0.0061)$. No significant differences were observed between the survival curves of patients with weak or strong FHIT protein staining (median survival of $208 \pm 17$ vs. $234 \pm 34$ days, 
$\mathrm{p}=0.665)$. Further, the proportion of FHIT protein-positive tumor cells was related to survival. Patients with tumors of less than $25 \%$ FHIT protein positive cells had the worst survival of $155 \pm 21$ days compared to $217 \pm 19$ days for patients with a proportion of $>25 \%$ of FHIT protein-expressing tumor cells $(\mathrm{p}=0.0016)$. Multivariate analysis using Cox regression including 11 variables confirmed the independent prognostic significance of FHIT protein expression next to performance status, tumor stage and LDH.

\section{P617}

\section{Vinorelbine and Carboplatin in Elderly Patients with Metastatic Non-Small Cell Lung Cancer (NSCLC): A Multicentre Phase II Study}

Deppermann K.-M. ${ }^{1}$ Ukena D. ${ }^{2}$, von Pawel J. ${ }^{3}$, Koschel G. ${ }^{4}$ Reck M. ${ }^{5}$, Serke M. ${ }^{6}$, Huber R.M. ${ }^{7}$, Eberhardt W. ${ }^{8}$, Niederle N. ${ }^{9}$ on behalf of the Arbeitsgemeinschaft Internistische Onkologie (AIO) ${ }^{1}$ Ruppiner Kliniken, Neuruppin, Germany, ${ }^{2}$ Universitätskliniken des Saarlandes, Homburg, Germany, ${ }^{3}$ Asklepios Fachkliniken, Gauting, Germany, ${ }^{4}$ Allgemeines Krankenhaus Harburg, Hamburg, Germany, ${ }^{5}$ Krankenhaus Großhansdorf, Großhansdorf, Germany, ${ }^{6}$ Lungenklinik Heckeshorn, Berlin, Germany, ${ }^{7}$ Ludwigs-Maximilian-Unviversität München, München, Germany, ${ }^{8}$ Klinikum der Universität DuisburgEssen, Innere Medizin (Tumorforschung), Essen, Germany,

${ }^{9}$ Klinikum Leverkusen, Leverkusen, Germany

Purpose: More than $60 \%$ of cancer patients (pts) are older than 65 years. Lung cancer is the leading cause of cancer related mortality in industrialized countries. Viniorelbine has been known as an active and well-tolerated treatment in pts older than 70 years. In younger patients, however, platinum containing combinations are more effective than monotherapies. The aim of this phase II study was to prospectively evaluate the toxicity and efficacy of the platinum-combination vinorelbine/carboplatin in elderly patients with metastatic NSCLC. Methods: Chemotherapy-naïve pts (age $>70$ years, stage IV NSCLC, Karnofsky $>80 \%$ ) were eligible for this investigation. They received vinorelbine $\left(25 \mathrm{mg} / \mathrm{m}^{2}\right.$ days 1 and 8$)$ and carboplatin (AUC 5 day 1$)$ every 3 weeks. Depending on response, a maximum of 6 chemotherapy cycles were administered. Results: 72 patients (female/male: 23/49) at median age of 75 (range 70-82) years were enrolled. Histology: 50\% adenocarcinoma, $33 \%$ squamous cell, $10 \%$ large cell, $7 \%$ others. $61 \%$ of metastases were located in the lung and lymph nodes and $64 \%$ of patients had more than one metastatic site. Karnofsky Index: $100 \% \mathrm{n}=6 ; 90 \% \mathrm{n}=35 ; 80 \% \mathrm{n}=31$. A total of 262 chemotherapy cycles were administered to 71 patients. 71 pts were evaluable for toxicity and 58 pts were evaluable for response. Best overall response: $21 / 58$ PR (36\%), 24/58 SD (41\%), 13/58 PD (23\%). Tumor control rate $45 / 58(78 \%)$. The main non-hematological toxicities were pain and dyspnoe. Grade 1-2 toxicities (\% of pts): anemia 84\%, neutropenia $15 \%$, thrombopenia $32 \%$, pain $50 \%$, dyspnoe $46 \%$, fatigue $45 \%$, coughing $38 \%$, neurotoxicity $6 \%$, Grade $3-4$ toxicities (\% of pts): anemia $11 \%$, neutropenia $59 \%$, thrombopenia $11 \%$, pain $18 \%$, dyspnoe $17 \%$, neurotoxicity $3 \%$. Fatigue or coughing grade 3-4 did not occur. Survival data will be presented at the meeting. Conclusion: The combination of vinorelbine and carboplatin shows a promising activity in elderly patients with stage IV NSCLC. The low toxicity profile favours out-patient treatment.

\section{P618}

Operable Stage IIIA NSCLC: Prospectively Randomized Multicenter Trial of Surgery and Postoperative Radiotherapy verus "Trimodality Treatment"-Analysis of Surgical Results and Toxicity

Korfee S. ${ }^{1}$, Gauler T. ${ }^{1}$, Pöttgen C. ${ }^{2}$, Stuschke M. ${ }^{2}$, Stamatis G. ${ }^{3}$, Wagner $H^{4}{ }^{4}$, Passlick B. ${ }^{4}$, Petersen $V^{5}$, Seeber S. ${ }^{1}$, Eberhardt W. ${ }^{1}$ Innere Klinik und Poliklinik (Tumorforschung), Klinikum der Universität Duisburg Essen, Germany, ${ }^{2}$ Klinik und Poliklinik für Radiologische Onkologie, Klinikum der Universität Duisburg Essen, Germany, ${ }^{3}$ Thoraxchirurgische Abteilung, Ruhrlandklinik Essen Heidhausen, Germany, ${ }^{4}$ Asklepios Fachklinik, München Gauting, Germany, ${ }^{5}$ Klinikum rechts der Isar, München, Germany

Purpose: selected patients (pts) with early stage IIIA-NSCLC (operable), 1-2 LN at mediastinoscopy, no clinical N2, no bulky/extranodal desease) are still taken to upfront surgery $(\mathrm{S})$ at most centers in Europe and North America We have compared this approach to an aggressive trimodality therapy -induction chemotherapy $(\mathrm{CTx})$, preoperative CTx/RTx and S- in a multicenter randomized German Krebshilfe trial*. Methods: Pts with operable IIIA (criteria as given above, mediastinoscopy, central T3N0-1, WHO 0,1) were stratified ( $\mathrm{TN}$-group, treatment center) and randomized: arm $\mathrm{A}-\mathrm{S}$ and postop RTx (50-60 gy); arm B - induction CTx (3 x cisplatin (P) $60 \mathrm{mg} / \mathrm{m}^{2} \mathrm{~d}$ 1+7/etoposide (E) $150 \mathrm{mg} / \mathrm{m}^{2} \mathrm{~d}$ 3,4,5) + cc CTx/RTx (45 Gy; 1.5 Gy bid; 1 x P $50 \mathrm{mg} / \mathrm{m}^{2} \mathrm{~d} 2+9$, E $\left.100 \mathrm{mg} / \mathrm{m}^{2} \mathrm{~d} 4,5,6\right)+\mathrm{S}(+\mathrm{PCI}-15$ x 2 Gy $=30$ Gy q 3 wks). Results: From 11/94 till 7/2001 overall 112 pts randomized, 6pts (3 per arm) not evaluable (mis-staging, pts refusal, early progression prior any study treatment). 106 pts were evaluable for complete study analysis: Pts characteristics M 90 F 16; age median 59 (37-71); histopathology SCC 49 adeno-ca 35 LCC 16 other 6; arm A: $\mathrm{n}=51$ pts T3N0-1 3 T1-2N2 38 T3N2 10; arm B: $\mathrm{n}=$ 55 pts T3N0-1 3 T1-2N2 45 T3N2 7. Arm A: S 51/51 probatory thoracotomy (PT) 5/51 R1/2 10/51 complete resection (R0) 36/51; Arm B: S 40/55 PT 1/55 R1/2 6/55 R0 33/55; peri- and postoperative morbidity and mortality not significantly different between both arms (rate of early deaths, infections, stump insufficiences, bleeding, embolisms, postop duration of hospitalization, time on postop respirator, time on intensity care unit). Conclusions: This trimodality protocol was proven feasible and safe in the given multicenter setting. S following this complex bimodality induction does not show increased morbidity or mortality rates. A detailed analysis of long-term survival data will be available at the time of the meeting 10/04). Although initially planned as phase-III trial, the small number of pts in this carefully selected pts group ("operable IIIA") makes this trial more a "multicenter randomized phase-II design" with a unique comparison (local treatment only versus aggressive trimodality (+PCI!))

*This trial was sponsored by a full trials grant from GERMAN KREBSHILFE, BONN

\section{P619}

Improved Overall Survival by Second-Line Combination

Chemotherapy: Follow-up Results of a Phase III Study Comparing Three-Weekly Paclitaxel/Carboplatin (PC) with Weekly PC in Patients (pts) with Advanced, Measurable Non-Small Cell Lung Cancer (NSCLC)

\section{Schroeder M. ${ }^{1}$, Dittrich $I^{2}{ }^{2}$, Ukena $D .^{3}$, Schütte $W$.}

${ }^{1}$ St. Johannes-Hospital, Duisburg, Germany, ${ }^{2}$ Lungenklinik Lostau, Germany, ${ }^{3}$ Universitätsklinik Homburg/Saar, Germany, ${ }^{4}$ Städtisches Krankenhaus Martha-Maria, Halle-Dölau, Germany

Background: PC is a widely-used active regimen for stage IIIB/IV NSCLC Previously we reported our results comparing weekly (A) with three-weekly PC (B) $1^{\text {st }}$-line therapy (A: paclitaxel (P) $100 \mathrm{mg} / \mathrm{m}^{2}, 3 \mathrm{~h}$ i.v. plus carboplatin (C) AUC 2, 30-60 min i.v. d1, 8, 15, 22, 29, 36 (q8w) for a maximum of two treatment blocks. B: P $200 \mathrm{mg} / \mathrm{m}^{2}$, 3h i.v. d1; C AUC 6, 30-60 min i.v. d1 for max. 6 courses). (D. Ukena, M. Schroeder, I. Dittrich et al; Proc ASCO 22 (2003), p. 623, A2506). Purpose: Hereby we present interim results of a retrospective follow-up analysis. We aimed to investigate the extent and survival impact of $2^{\text {nd }}$-line chemotherapy regimens used today in large community hospital settings. Patients and methods: 804 pts participated in our phase III study; recruitment started in July 1998. Follow-up exams were regularly carried out in three-monthly intervals after end of $1^{\text {st }}$-line therapy. Choice of further therapy options was at the physicians' discretion.

537 patients underwent the first follow-up exam; 267 pts had died. The majority of pts alive presented with a suitable performance status (ECOG 0 39 pts; 1: 201 pts; 2: 132 pts). Among all pts, $25.9 \%$ (208 pts) obtained $2^{\text {nd }}$. line chemotherapy, 101 of them had received PC q3w, 107 weekly PC before. For 104 pts $(50 \%)$, physicians opted for $2^{\text {nd }}$-line mono-therapy; the other half was treated by duplet combinations. With regard to former $1^{\text {st }}$-line therapy, both treatment options were equally balanced in the two study arms.

Results: Patients treated by $2^{\text {nd }}-$ line combination therapy showed in comparison to $2^{\text {nd }}$-line mono-therapy an improved overall survival in terms of median survival (13.8 months vs. 12.8 months), 1-year (61/104, 58.6\% vs. 54/104, $51.9 \%)$ and 2 -years survival rates $(17 / 104,16.4 \%$ vs. $12 / 104,11.5 \%)$. The observed survival advantage is in line with the response rates determined so far: $8 \%$ vs. $3 \%$ responses (CR/PR), $29 \%$ vs. $17 \%$ disease stabilisation. Similar results are obtained if the subgroups in the $\mathrm{q} 1 \mathrm{w}$ and $\mathrm{q} 3 \mathrm{w}$ study arm are compared. Discussion: In recent literature, a benefit in terms of survival as well as quality of life has been demonstrated for $2^{\text {nd }}$-line mono-therapy compared to best supportive care. Our results show that approximately one quarter of patients received $2^{\text {nd }}$-line chemotherapy after preceding $\mathrm{PC}$ therapy and indicate that for $2^{\text {nd }}$-line therapy the use of combination regimen compared to mono-therapy offer a distinct clinical benefit by improving 1 year and 2-years survival rates. 
P620

\section{Bendamustin in Relapsed Small Cell Lung Cancer: A Phase II Study}

\section{Schmittel A. ${ }^{1}$, Schulze $K^{2}{ }^{2}$, Thiel E. ${ }^{1}$, Keilholz U. ${ }^{1}$}

${ }^{1}$ Charité Universitätsmedizin Berlin Campus Benjamin Franklin, Medizinische Klinik III (Hämatologie, Onkologie und Transfusionsmedizin), Berlin, Germany, ${ }^{2}$ Charité Universitätsmedizin Berlin Campus Benjamin Franklin, Medizinische Klinik II (Kardiologie und Pulmologie), Berlin, Germany

Purpose: Bendamustin has single agent activity in first line therapy of small cell lung cancer (SCLC) with a response rate of $40-50 \%$ with moderate toxicity. We investigated the activity of bendamustin in patients, who relapsed $>$ 2 months after first line treatment (sensitive relapse). Methods: Bendamustin was administered at a dose $120 \mathrm{mg} / \mathrm{m}^{2}$, on day $1+2$ of a three week cycle. In the absence of tumor progression a maximum of six cycles was given Response was assessed after the second, forth and sixth cycle. Inclusion criteria were absence of brain metastases and a time to relapse $>2$ months after first line chemotherapy. Patients refractory to first line therapy were excluded from this study. Results: Fourteen patients are currently evaluable for interim analysis. Five out of fourteen $(36 \%)$ patients had a confirmed partial response. Four (27\%) had stable disease and 4 (27\%) progressed. One patient was not evaluable for response analysis due to early death. Grade IV neutropenia occurred in two (14\%) patients, of these one patient had fata neutropenic sepsis. Main non-hematologic toxicity was mild nausea. No alopecia was observed. In these 14 patients median TTP was 6 months (95\% CI 1.8-10.2) and median survival was 7 months (95\% CI 5.9-8.1). Conclusions: This interim analysis already demonstrates activity of bendamustin in relapsed SCLC. The study is ongoing until 35 patients are included.

\section{P622}

Weekly Irinotecan in Patients with Advanced Non-SmallCell Lung Cancer (NSCLC) after Failure of Cisplatin, Taxane and Gemcitabine Based Chemotherapy

\section{Schneider A., Schmiedl S., Brinkmann C., Hense J., Seeber S.}

Dept. of Internal Medicine (Cancer Research), West German Cancer

Center, University of Essen Medical School, Essen, Germany

Purpose: Irinotecan is known to be active in patients with NSCLC since its discovery in the early 90 s but failed to achieve approval or widespread use outside clinical studies. Patients and Methods: We reviewed the data of all patients of our department with advanced NSCLC who received irinotecan between 2001 and 2004. 21 patients, 20 male and one female, median age 58 years (range 42-76y), 17 with adeno, 3 squamous cell and 1 mixed cell carcinoma were treated with an average of 4 different chemotherapies prior to irinotecan. Patients had progressive disease on cisplatin or carboplatin (21/21), paclitaxel or docetaxel (19/21; best response prior to PD: 5 PR, 4 SD), gemcitabine (20/21; 1 PR, 4 SD) and vinorelbine (18/21). Irinotecan was given at a weekly dose of $100 \mathrm{mg} / \mathrm{m}^{2}$ for 6 weeks with one week rest until disease progression. Results: Patients received an average of 11 weekly irinotecan doses. Four patients discontinued therapy due to toxicity and were not evaluable for response. Among the 17 evaluable patients there were 3 partial remissions (18\% PR) and 7 patients with stable disease (41\% SD) resulting in a clinical benefit rate of 59\%. There was no grade 3 or 4 leucopenia, thrombocytopenia or stomatitis. Seven patients had grade 3 or 4 diarrhoea. Time to treatment failure was 3,6 months (range 1 to 11 months). Median survival from time of metastatic disease for these patients was 24 months. Conclusions: Weekly irinotecan is effective in patients with advanced NSCLC and response rates compared favourably to those of prior gemcitabine or taxane based chemotherapy in these heavily pretreated patients. Disease stabilization was seen in $59 \%$ of all patients.

The use of the increased number of active substances for sequential treatment of patients with NSCLC achieved valuable symptom control and may lead to increased survival of patients with incurable disease.

\section{P623}

\section{Dynamic Fluoro-Desoxy-Glucose Positron Emission Tomography for the Prediction of Chemotherapy Outcome in Non Small Cell Lung Cancer}

Hoffmann M. ${ }^{1}$, Dimitrakopolou-Strauss A. ${ }^{2}$, Dingeldein $G .{ }^{1}$, Henrich D. ${ }^{1}$, Landmann T. ${ }^{1}$, Schanz J. ${ }^{1}$, Strauss L. G. ${ }^{2}$, Uppenkamp $M$.

${ }^{1}$ Medizinische Klinik A, Klinikum der Stadt Ludwigshafen gGmbH, Ludwigshafen am Rhein, Germany, ${ }^{2}$ Klinische Kooperationseinheit Nuklearmedizin, Deutsches Krebsforschungszentrum, Heidelberg, Germany

Purpose: The Fluoro-Desoxy-Glucose positron emission tomography (FDGPET) provides detailed information about the biology of a tumour and its change during treatment with chemotherapy. We evaluated the parameters of the FDG kinetic model in patients with metastatic NSCLC and assessed their value with regard to prediction of treatment outcome. Methods: We evaluated ten patients with advanced non small cell lung cancer (NSCLC). Nine patients received a chemotherapy consisting of Oxaliplatin and Vinorelbine, one patient was treated with a combination of Carboplatin and Vinorelbine. The patients received chemotherapy for six cycles of chemotherapy or until progressive disease. Three FDG-PET-studies were carried out (1. prior to the chemotherapy, 2. after the first course of chemotherapy, 3. after the third course). The clinical follow-up data after three cycles chemotherapy and a WHO-based classification (progressive disease (PD), stable disease (SD), partial remission (PR), complete remission (CR)) served as reference for the PET data. The following parameters were retrieved from the dynamic PET studies: SUV, fractal dimension (FD), two compartment model with computation of k1, k2, k3, k4 and the vessel density (VB). We divided into two groups, PR/NC and SD. Results: Response rates by conventional staging were as follows: PR - one patient, SD - two patients, PD - seven patients. The best correct classification rate by FDG-PET was obtained using al kinetic data of the first study. The SUV of the first study was superior to all other parameters as a single one with respect to the overall CCR. The CCR for SUV was followed by the FD, the $\mathrm{k} 3$ and by the influx for the prediction of therapy outcome. Influx and SUV of the second study were comparable with respect to therapy outcome. $\mathrm{PR} / \mathrm{NC}$ was associated with a higher k1 and a higher VB of the first study. Conclusions: The results of this ongoing study demonstrate, that compartmental and non-compartmental analysis of the FDG metabolism are helpful for the prediction of therapy outcome and superior to the use of SUV alone.

P624

Systemic Treatment and Survival in Patients with Advanced and Metastatic Non-Small Cell Lung Cancer (NSCLC): A Retrospective Analysis from a Lung Cancer Center

\section{Martens U.M. ${ }^{1}$, Kaiser P. ${ }^{1}$, Bludau P. ${ }^{1}$, Schulte Mönting J. ${ }^{2}$, Digel $W$.}

${ }^{1}$ Medical University Center, Dep. for Hematology/Oncology, Freiburg, Germany, ${ }^{2}$ Institute for Medical Biometry and Informatics, University of Freiburg, Germany

Purpose: Non-small cell lung cancer (NSCLC) accounts for approximately $80 \%$ of all lung cancers, with more than one million new cases worldwide each year. Most patients present with locally advanced stage III or metastatic stage IV disease. Chemotherapy for these patients has been palliative and treatment outcome remains poor. The survival rates have been improved by newer generation agents combined with cisplatin ranging from 8 to 10 months. Methods: We performed a retrospective analysis of NSCLC patients stage IIIB and IV treated with systemic chemotherapy between 1993 and 2003 in our center. Systemic chemotherapy included following protocols: Vinorelbin/Carboplatin; Gemcitabine/Cisplatin; Taxol/Carboplatin; Taxotere/ Cisplatin; Taxotere mono; Taxol mono; Vinorelbin mono; and Gemcitabine mono. Results: Eligible for evaluation were 358 patients (squamous cell carcinoma, $\mathrm{n}=108$; adenocarcinoma, $\mathrm{n}=191$; and other non-classified carcinoma, $\mathrm{n}=59)$. A total of 237 patients $(66.2 \%)$ had metastatic disease. Interestingly, the rate of brain metastasis was strikingly high (21.2\%) including $9.2 \%$ of patients with initial metastasis and $12 \%$ following treatment course. The efficacy of chemotherapy was as follows: 


\begin{tabular}{|c|c|c|c|}
\hline Response & $\begin{array}{c}\text { First-line } \\
(\boldsymbol{n}=\mathbf{3 5 1})\end{array}$ & $\begin{array}{c}\text { Second-line } \\
(\boldsymbol{n}=\mathbf{1 9 3})\end{array}$ & $\begin{array}{c}\text { Third-line } \\
(\boldsymbol{n}=\mathbf{9 0})\end{array}$ \\
\hline $\mathrm{CR}$ & $5(1.4 \%)$ & $1(0.5 \%)$ & $1(1.1 \%)$ \\
\hline $\mathrm{PR}$ & $73(20.8 \%)$ & $20(10.4 \%)$ & $5(5.6 \%)$ \\
\hline $\mathrm{SD}$ & $197(56.1 \%)$ & $134(69.4 \%)$ & $63(70 \%)$ \\
\hline $\mathrm{PD}$ & $76(21.7 \%)$ & $38(19.7 \%)$ & $21(23.3 \%)$ \\
\hline
\end{tabular}

The median survival for all patients was 12.9 months, for patients with squamous cell carcinoma 13.1 months, for patients with adenocarcinoma 13.7 months and for all other 10 months. Conclusions: In summary, multiple chemotherapy treatments for advanced and metastatic NSCLC results in survival rates which are superior compared to historic controls. Third-line chemotherapy protocols may improve the outcome in some patients. The response rates of different treatment regimes will be presented in detail.

\section{P625}

\section{Measuring PKC- $\beta$ Expression in NSCLC}

\author{
Sandusky G. ${ }^{1}$, Ballard D. ${ }^{1}$, Mintze K. ${ }^{1}$, Thornton $D .{ }^{2}$, \\ McClelland $P^{3}{ }^{3}$, Ma D. ${ }^{3}$, Niyikiza $C^{3}{ }^{3}$, Lahn M. ${ }^{2}$ \\ ${ }^{1}$ Discovery Pathology - Eli Lilly and Company, Indianapolis, USA, \\ ${ }^{2}$ Medical Oncology - Eli Lilly and Company, Indianapolis, USA, \\ ${ }^{3}$ Oncology Product Team Pharmacogenomics - Eli Lilly and \\ Company, Indianapolis, USA
}

Purpose: Intracellular signaling proteins have recently been recognized as targets in cancer. One such intracellular signaling protein is PKC- $\beta$ an isoform of protein kinase C. LY317615 (Enzastaurin $\mathrm{HCl}$ ) is a potent and selective inhibitor of PKC- $\beta$ and has shown potent anti-angiogenic activity in preclinical animal models. While a number of pre-clinical studies suggest that enzastaurin has anti-tumor effect and is well tolerated in animal studies, the expression of PKC- $\beta$ in NSCLC has not been extensively studied. Methods: We here present an immunohistochemistry (IHC) procedure and a procedure to determine mRNA levels of PKC- $\beta$ II protein and gene expression in paraffin-embedded material of NSCLC biopsy samples. Results: In 42 specimens of NSCLC of patients with stage I and II disease, 28 specimen were adenocarcinoma and 14 were squamous cell carcinoma. In $65 \%$ of these NSCLC samples, PKC- $\beta$ II was expressed in tumor and tumor-adjacent tissue Interestingly, staining was higher in tumor-associated tissue, especially in inflammatory cell infiltrates and tumor-associated vessels. Also, squamous cell carcinoma appeared to express higher levels of PKC- $\beta$ II than adenocarcinoma (4/14 $3+$ tumors vs. 2/28, respectively). Furthermore, the same paraffin-embedded specimen used for IHC, were also employed to determine mRNA gene expression of PKC- $\beta$. This different technique confirmed that PKC- $\beta$ is expressed in tumor cells and future studies will specifically look at its expression in tumor-associated vessels. Conclusions: In summary, we here show a reliable technique to determine PKC-bII levels in NSCLC using IHC and compare it to detection methods using mRNA gene expression.

\section{P626}

\section{Assessment of Costs and Outcomes of Chemotherapy in an Observational Setting in Patients with Advanced NSCLC (ACTION)}

Bischoff H.G. ${ }^{1}$, Thomas . $^{2}$, Hamann M. ${ }^{3}$, Leschinger M.I. ${ }^{3}$, Steppert C. ${ }^{4}$, Fischer J.R. ${ }^{5}$, Schneider C. $-P .{ }^{6}$ on behalf of the ACTION study group

${ }^{1}$ Thoraxklinik Heidelberg gGmbH, ${ }^{2}$ Universitätsklinik Münster,

${ }^{3}$ Lilly Deutschland GmbH Bad Homburg, ${ }^{4}$ Bezirksklinikum Obermain

Ebensfeld, ${ }^{5}$ Klinik Löwenstein, ${ }^{6}$ Zentralklinik Bad Berka

Decision makers in the health care system are increasingly asked to include total treatment costs into their therapeutic decisions. Treatment of NSCLC patients and clinical outcome could be improved during the last years. Especially newer agents, like gemcitabine, showed significant survival benefit and improved quality of life. Available data concerning total treatment costs for these newer agents currently only exist from retrospective assessments of large randomized, controlled clinical trials. Therefore clinical data from routine clinical care settings associated with related total treatment costs are of high interest.

ACTION, an observational trial, collects data of patients with advanced NSCLC (stage IIIB/IV) in an open, prospective setting. Treatment decisions and patients characteristics will be documented as well as chemotherapies used, toxicities and quality of life for a period of 18 months. The documentation includes 7 points in time during therapy, collecting data for first and second line treatment, and fixed points in time. Standard clinical outcome measures like tumor response or time to disease progression will be reported and more patient orientated measures like "Days spend outside the hospital" or "Complication free days" as well. Finally related total costs will be assessed to evaluate the benefit of newer chemotherapy agents in the treatment of NSCLC, documenting every day's use.

ACTION is carried out in 5 European countries with over 800 patients enrolled (status of April 2004). In Germany currently 574 patients are enrolled in 86 centers reflecting a high interest in total treatment cost data in the light of a changing health care system in the hospital domain to a diagnoses related groups (DRG) system. Analyses of baseline data will be performed and presented at the congress.

\section{Poster Session: Breast Cancer}

P627

\section{Detection of Osteolytic Lesions in an Animal Model of Human Breast Cancer Metastasis with Flat-Panel Detector-Based Volumetric Computed Tomography}

Missbach J. ${ }^{1}$, Obenauer S. ${ }^{2}$, Rieker J. ${ }^{2}$, Huang $Y{ }^{4}$, Kauer $F^{3}$, Grabbe E. ${ }^{2}$, Trümper L. ${ }^{1}$, Vogel W.F. ${ }^{4}$, Alves $F^{1}{ }^{1}$

${ }^{1}$ Dept. of Haematology/Oncology, ${ }^{2}$ Dept. of Diagnostic Radiology, ${ }^{3}$ Dept. of Traumatology, University of Goettingen, Germany, ${ }^{4}$ Dept. of Laboratory Medicine and Pathobiology, University of Toronto, Canada

A major limitation in evaluating tumor progression in murine tumor models is the inability to detect and monitor tumor metastasis in detail over the course of disease. Here, implantation of human breast cancer MDA-MB-231 cells, which is a well characterized mouse model for bone metastasis, was used to perform imaging studies with a flat-panel detector-based cone beam volume computed tomograph (FPD-VCT). The present study discusses the utility of a new imaging technique of high resolution FPD-VCT in detecting bone metastasis in an animal model of breast cancer metastasis. Ten week old female SCID mice $(n=8)$ were intracardially injected with $5 \times 10^{5}$ human breast cancer MDA-MB-231 cells. After 4 weeks, mice were sacrificed and front and hind legs were dissected. The destruction of bone tissue by metastatic cells was imaged using a FPD-VCT (General Electrics Prototype). Additionally, the legs were analysed by Faxitron X-ray radiographic inspection (Hewlett \& Packard, Faxitron model 43855). For histological analysis the legs were decalcified and paraffin sections were stained with H\&E. Imaging studies by FPD-VCT demonstrated the development of distinct radiolucent metastatic osteolytic lesions in distal femur, proximal tibia, and proximal humerus in six bones of three mice at day 28 after cell inoculation. These osteolytic cavities could be visualized three dimensionally allowing volumetric measurements, which were within the range of $0.35-0.63 \mathrm{~mm}^{3}$. The presence of these osteolytic lesions was confirmed by standard radiographic examination (Faxitron). Osteolysis within the diaphysal cortical bone of one humerus was only detected by FPD-VCT. Histological examination revealed metastatic MDA-MB 231 breast cancer cells in the bone marrow cavity.

In conclusion, FPD-VCT shows excellent sensitivity and accuracy in detecting skeletal lesions and can therefore be used to image the localisation of bone metastasis including osteolytic lesions within the diaphysal cortical bone. In comparison to standard Faxitron x-ray FPD-VCT can be applied in vivo and obtains true isotropic $3 \mathrm{D}$ volume image data sets for evaluation in arbitrary planes. Due to precise volumetric measurement of bone metastasis, FPD-VCT will be a powerful tool to monitor tumor progression in vivo and response to anti-cancer therapies over the course of disease. 
P628

\section{Gemcitabine and Mitoxantrone in Metastatic Breast Cancer: A Phase-I Study}

\section{Schmid $P .{ }^{1}$, Flath B. ${ }^{1}$, Akrivakis A. ${ }^{2}$, Heilmann $V .{ }^{3}$,} Mergenthaler H.G. ${ }^{4}$, Sezer O. ${ }^{1}$, Kreienberg . $^{3}$, Possinger K. ${ }^{1}$ ${ }^{1}$ Medizinische Klinik mit Schwerpunkt Onkologie und Hämatologie, Charité Campus Mitte, Humboldt Universität zu Berlin, Germany, ${ }^{2}$ Department of Oncology and Hematology, Ernst von Bergmann Klinik, Potsdam, Germany, ${ }^{3}$ University of Ulm, Department of Gynecology, Ulm, Germany, ${ }^{4}$ Department of Oncology and Hematology, Katharinenhospital, Stuttgart, Germany

Purpose: Gemcitabine and mitoxantrone are active agents for the treatment of metastatic breast cancer. Due to different modes of action and a favorable toxicity profile they are suitable for combination therapy. This phase I tria was therefore initiated to determine the optimal doses for the combination in patients with metastatic breast cancer. Secondary objectives included the evaluation of the safety and efficacy of the regimen. Patients and Methods: Patients with metastatic breast cancer were treated with gemcitabine (1000$\left.1400 \mathrm{mg} / \mathrm{m}^{2}\right)$ on days 1,8 and 15 and mitoxantrone $\left(10-14 \mathrm{mg} / \mathrm{m}^{2}\right)$ on day 8 . Treatment was repeated every 4 weeks for a maximum of 8 cycles. Doses were assigned at registration according to the escalation scheme. Results Twenty-six patients received a total of 93 cycles at 5 different dose levels. The maximum tolerated doses were $1200 \mathrm{mg} / \mathrm{m}^{2}$ gemcitabine and $14 \mathrm{mg} / \mathrm{m}$ mitoxantrone with grade 4 neutropenia being the dose limiting toxicity. Recommended phase II doses, however, are gemcitabine $1200 \mathrm{mg} / \mathrm{m}^{2}$ and mitoxantrone $12 \mathrm{mg} / \mathrm{m}^{2}$ based on a similar median dose intensity and a more favorable toxicity profile. Predominant toxicity was myelosuppression with grade 3 or 4 leukopenia, neutropenia and thrombocytopenia in $43.0 \%, 37.6 \%$ and $10.8 \%$ of courses, respectively. Non-hematologic toxicity was generally mild. Most common toxicities were nausea, vomiting, alopecia and elevation of liver enzymes. Twenty-one patients were assessable for response. Four patients achieved a partial response accounting for an overall response rate of 19\%. In addition, 12 patients (57\%) had stable disease and 5 patients (24\%) failed to response to the treatment. Median duration of response and duration of clinical benefit were 14 and 9 months, respectively. Conclusion: In this phase I study of gemcitabine and mitoxantrone, DLT was neutropenia. Recommended phase II doses are gemcitabine $1200 \mathrm{mg} / \mathrm{m}^{2}$ and mitoxantrone $12 \mathrm{mg} / \mathrm{m}^{2}$

\section{P629}

\section{Breast Cancer and Retroviruses: Re-examinating the Postulate Using a Retrovirus-specific DNA Chip}

\section{Seifarth W. ${ }^{1}$, Greenwood A. ${ }^{2}$, Hehlmann $R .^{1}$, Leib-Mösch $C^{1,2}$}

${ }^{\prime}$ III. Medizinische Universitätsklinik Mannheim der RuprechtKarls-Universität Heidelberg, Mannheim, Germanyl,

${ }^{2}$ GSF-Forschungszentrum für Umwelt und Gesundheit, Institut für Molekulare Virology, Oberschleißheim, Germany

Purpose: The mouse mammary tumor virus (MMTV) is the aetiologica agent of neoplasia in the murine mammary gland. Recently, it has been reported that a human (MMTV)-like retrovirus (HMLV) occurs in human breast cancer cell lines as well as in 38\% of human breast cancer samples. However, transcripts of human MMTV-like endogenous retroviral sequences, HERV-K(HML) as part of our genetic make-up detectable in all human cells makes reliable discrimination of a putative MMTV-related infectious retrovirus difficult. Since the aetiology of most breast cancers is unknown, it is important to test the hypothesis of a retroviral disease-associated agent and therefore, we set out to investigate a breast cancer population in our locality for MMTV/HMLV and HERV transcriptional activity. Methods: Tissue samples derived from 21 patients with mammary carcinoma (all invasive, with intraductal component) were analyzed. For each patient a pair of samples, exclusively corresponding to normal and malignant cells was analyzed for retroviral transcripts. After preparation of total-RNA and in vitro synthesis of cDNA a novel high throughput assay for comprehensive analysi of retroviral activity in biological samples was employed (Seifarth et al 2003). The qualitative test combines multiplex polymerase chain reaction (PCR) using fluorochrome (Cy3)-modified primer cocktails and glass DNA chip hybridization. It allows detection as well as identification of most known retroviral reverse transcriptase (RT)-related nucleic acids in one single experiment. Results: Distinct members of human MMTV-like and type C-related HERV elements have been found to be transcribed in normal cells of the mammary gland in a tissue-specific manner. In most cases the same expression pattern was observed in the corresponding malignant tissue samples. For some elements ((HERV-K(HML-4), HERV-K(HML-3), ERV-FRD) an downregulation of HERV transcription in the corresponding tumor samples was observed $(\mathrm{p}=0.0008)$. Conclusion: In the human mammary gland a distinct set of HERV elements is transcriptionally active in a tissue-specific manner. Despite the high sensitivity and species specificity of the assay we found no evidence that MMTV/HMLV pol transcripts genetically distinct from HERVs are present in clinical tissue samples collected in our locality.

\section{P630}

\section{Human Plasma Facilitates Enrichment of Circulating Epithelial Cells from Peripheral Blood}

\section{Schwalbe M., Jörke C., Hauschild F., Höffken K., Pachmann K. Clement J.H. \\ Dept. Internal Medicine II, Friedrich-Schiller-University Jena, Germany}

Purpose: We could show recently, that magnetic nanoparticles interact with viable cells in a cell-type specific manner. In order to optimize the differential labeling of tumor cells and leukocytes, we have studied the role of plasma as an important fraction of human blood. Methods: Leukocytes were prepared by erythrocyte lysis from whole blood samples of healthy volunteers for in vitro experiments and from whole blood samples from patients. The breas cancer cell line MCF-7 was kept under standard cell culture conditions. Cells were inoculated with magnetic core/carboxymethyl-dextran nanoparticles with an average magnetite/maghemite core TEM-size varying between 3 and $15 \mathrm{~nm}$. Magnetically labeled cells were separated by MACS. The separated cells were analyzed by FACS and Laser Scanning Cytometry (LSC). Tumor cells were detected with anti-human epithelium-antigen (HEA)-FITC Results: Whole blood samples from tumor patients were treated with erythrocyte lysis buffer to enrich the leucocyte fraction and to remove the plasma fraction. The remaining cells were incubated with magnetic nanoparticles in the presence of none, $1 \%$ or $5 \%$ plasma for 8 min and separated with our regime. The retained cells (positive fraction) and the flow-through cells (negative-fraction) were quantified. The leukocyte fractions showed a considerable reduction in binding of nanoparticles already after addition of $1 \%$ plasma. In the presence of $5 \%$ plasma less than $20 \%$ of the applied cells were separable by MACS. The amount of circulating epithelial cells within the positive and negative fraction was estimated by LSC. In contrast to normal leukocytes the epithelial cell fraction increased 2.5-fold in the presence of 5\% plasma in comparison to the sample without supplementation of plasma. The amount of epithelial cells enriched from patients samples kept constant at $75 \%$ compared to the unseparated control. Conclusion: We could show that human plasma affects the interaction of magnetic nanoparticles with leukocytes more seriously than with epithelial tumor cell lines. This is in concordance with previously reported results from in vitro experiments.

This work was supported by the DFG-Schwerpunktprogramm SPP1104, grant CL 202/1-1

\section{P631}

Radiofrequency Ablation (RFA) of Hepatic Metastases in Patients with Metastatic Breast Cancer (MBC): An Interim Analysis

\section{Helmberger T., Heinemann V., Vick C., Wallnöfer A., Gotz A.} Reiser $M$.

University of Munich, Germany

Background: A subgroup of MBC patients present hepatic metastasis without extrahepatic spread. Even with systemic therapy survival is limited. Since surgical therapy is not considered a standard of care in these patients, minimally invasive therapies as radiofrequency ablation (RFA) are gaining an increasing importance. Methods: Between 1999 and 2003, 32 patients (median age 62 years, range $41-76$ years) with syn- (5/32) and metachronous (27/32) solitary liver metastases from breast cancer were treated by RFA (Starburst XL, RITA Medical Systems, Mountainview, CA USA) subsequently/parallel to chemo and/or hormonal therapy. All procedures were performed under CT-fluoroscopical guidance applying conscious sedation and local anaesthesia. Number of lesions, primary success rate, complications, total and disease free survival were recorded and analyzed. Follow-up 
(by clinical, laboratory, and imaging studies) was achieved over up to 44 months. Results: RFA was performed between 0 to 138 months (median 37 months) after diagnosis of the metastasis. In 32 patients, 73 metastases were successfully treated by RFA without any major adverse event. 13/32 (40 \%) patients had a solitary metastasis, the rest had 2 to 5 metastases ( 8 had 2,2 had 3, 5 had 4, and 4 had 5). The size of the metastases ranged from 3 to 85 $\mathrm{mm}$ with a median diameter of $20.3 \mathrm{~mm}$. During follow-up of 1 to 44 month (median 17.2 months), 25 patients (79\%) remained desease-free in the liver, 1 patient $(3 \%)$ developed a local tumor recurrence, and 6 patients $(18 \%)$ new hepatic metastases. 9/32 (28\%) patients died 1 to 28 months after RFA due to extrahepatic tumor spread. Among the 7 patients who developed new lesions 6 underwent repeated RFA. Up to the present time of evaluation, the median survival after RFA has not been reached. Conclusion: RFA appears to be a valuable adjunct to the classic armamentarium of endocrine- and chemotherapy in hepatic metastasis of breast cancer and needs to be investigated in comparative studies.

\section{P632}

\section{How Can CEA and CA15-3 Be Used for Estimation of the Clinical Status and Effectiveness of Therapy During Metastatic Breast Cancer?}

\section{Stieber $P{ }^{1}{ }^{,}$, Lässig $D .^{1}$, Untch $M{ }^{2}$, Nagel D. ${ }^{2}$, Heinemann $V^{3}$}

${ }^{1}$ Klinische Chemie, Klinikum der LMU-München-Großhadern,

Germany, ${ }^{2}$ Frauenklinik, Klinikum der LMU-München-Großhadern,

Germany, ${ }^{3}$ Medizinische Klinik III, Klinikum der LMU-München-

Großhadern, Germany

Background: Tumor markers like CEA and CA 15-3 are often used in follow up care of breast cancer, but mainly due to a lack of knowledge, experience but also confidence- the actual situation is more to observe kinetics than to react on these results. Therefore we try to validate the significance of CEA and CA15-3 in the course of metastatic disease. Patients and methods: We performed our study in a group of 101 breast cancer patients who developed metastatic lesions. The patients mostly suffered from bone $(n=54)$, liver $(n=51)$, lung $(n=35)$ and lymph node metastases $(n=39)$. All patients were treated by systemic therapies. On the basis of changing therapeutic regimes we totally monitored 223 different courses of therapies including endocrine $(n=29)$, chemotherapeutic $(n=73)$, antibody $(n=7)$ and combined $(n=114)$ treatments. CEA (AxSYM/ Abbott) and CA15-3 (Elecsys/ Roche) were mostly determined every 3 or 4 weeks, the therapeutic response was assessed by UICC-criteria. Results: Meanwhile we evaluated CEA and CA15-3 at firs diagnosis of metastases and at every further moment of progression/new metastases. At first metastatic disease CEA was in 53\% above the 95th percentile of healthy individuals and CA $15-3$ in 74\% (19\% CEA and CA 153 negative) with enhancing percentage of sensitivity with increasing number of further events 'progressive disease (PD)' ( 2nd PD CEA 52\% / CA15-3 $69 \%$, 3rd PD $65 \% / 80 \%$, 4th PD 74\% / 93\%), decreasing number of false negatives (3\% 4th PD) and a general shift of the value levels of CEA (ng/ml)/ CA 15-3 (U/ml) with the number of events, e.g. the medians 1st PD CEA 2.7/ CA 15-3 43.4; 2nd PD $2.6 / 46.2$; 3rd PD 5.3 / 78.5; 4th PD $8.8 / 92.5$ ). Conclusion: Our results show a clear correlation between CEA /CA 15-3 and the number of events as well as effectiveness of therapy.

\section{P633}

\section{Ex-vivo Culture of primary Breast Cancer Specimens to Study Sensitivity to Anticancer Drugs in an Intact Tissue Environment}

Murdter T. ${ }^{1}$, van der Kuip $H^{1}{ }^{1}$, McClellan M. ${ }^{1}$, Gutzeit $S .{ }^{1}$ Simon $W^{2}$, Gerteis A. ${ }^{2}$, Fritz $P{ }^{2}{ }^{2}$, Aulitzky W.E. ${ }^{2}$

${ }^{1}$ Dr. Margarete Fischer Bosch Institut Für Klinische Pharmakologie, Stuttgart, Germany, ${ }^{2}$ Robert Bosch Krankenhaus, Stuttgart, Germany

Purpose: Current breast cancer model systems to study drug sensitivity such as cell lines, isolated tumor cells and animal models only poorly predict clinical response. This may be caused by the fact, that the drug response of tumor cells is greatly influenced by their environment. Therefore model systems which allow to study drug effects on primary tumor cells within their tissue environment may be a great advance for research on response prediction or the study of combinatorial strategies. Results: We have developped an ex vivo culture system for studying drug effects on tumor cells within their natural tissue context. $200 \mu \mathrm{m}$ thick tissue slices from freshly excised tumor samples were prepared and maintained under cell culture conditions. Within these slices the cells remained viable and continued to proliferate for at least 3 to 7 days. The different cell compartments were distinguishable using fluorescent-labeled antibodies recognizing specific surface markers. The tissue architecture did not change significantly during the culture period. The viability of cells was assessed in culture using a confocal laser microscopy and three color fluorescence viability assay. Conclusions This tissue culture system allows to examine cytotoxic drug effects and their molecular consequences in primary tumor samples withing their natural environment. This opens the window for extensive molecular studies on the biological effects of anticancer drugs.

P634

Humoral Immune Response in Breast Carcinoma Patients against Human Endogenous Retroviral Gag Protein Identified by Recombinant Yeast Antigen Surface Expression (RAYS)

Imig J., Wadle A., Bette B., Pfreundschuh M., Renner C. Department of Internal Medicine I, University of Saarland Medical School, Homburg/Saar

Purpose: In order to define novel antigens which might serve as potential targets for a tumor vaccination strategy we serologically screened a cDNAlibrary derived from mammary gland tumor tissues. One clone which was identified, coded for a human endogenous retroviral K (HERV-K) Gag protein. HERVs are relics from integration events of former exogenous retroviruses into the human genome. The Gag protein of exogenous retroviruses is part of the capsid, while the endogenous counterparts are often mutated and, therefore, not infectious. Until now, there are several indications pointing out an association of ERV-derived protein expression and tumorigenesis. In the present study we analysed the immunological response and mRNA expression of HERV-K Gag in serum and tissue samples of breast cancer patients Methods: Cloning and expression of breast carcinoma cDNA library in yeast was done by standard methods. Detection of the IgG antibody response against HERV-K Gag was done by RAYS analysis using serum samples from 100 breast cancer patients and 50 healthy controls. mRNA expression analysis of 12 tumor and four normal breast tissue samples was carried out using Real-time PCR. Results: We observed an HERV-K Gag serological B cell response in $11 \%$ of all patients with breast cancer. Only $3 \%$ of healthy controls had a weak titer. mRNA expression analysis confirmed that the expression level of HERV-K Gag was elevated in breast cancer tissues compared to the normal breast samples. Conclusions: Antibody responses to HERV-K Gag are found in a considerable proportion of breast cancer patients and might be due to protein overexpression. Further analysis is needed to link the expression and serological data to disease stage, tumor type and extent of disease.

\section{P635}

HER2 Positive Metastatic Disease in Primary not Overexpressing HER2 Breast Cancer: Re-Evaluation of HER2 Status and Tumor Marker Guided Therapy with Vinorelbine and Trastuzumab

\section{Stemmler H.J. ${ }^{1}$, Lässig $D .{ }^{2}$, Stieber $P{ }^{2}{ }^{2}$, Heinemann V.}

${ }^{1}$ Medizinische Klinik III, LMU München, Klinikum Grosshadern, Germany, ${ }^{2}$ Institut für Klinische Chemie, LMU München, Klinikum Grosshadern, Germany

Background: HER2 is overexpressed in 20-30\% of breast cancers Compared to chemotherapy alone, chemotherapy with trastuzumab improves clinical outcome in patients with HER2-positive metastatic breast cancer. In general, HER2 status in a primary lesion predicts the status of a metastatic lesion such that biopsy of metastatic lesions appears unnecessary. Patient and Method: We report a 39-year old women who was diagnosed with primary breast cancer wich has shown low HER2 expression using the method and scoring system of the DAKO Hercep Test (DAKO score 1+). After failure of several chemotherapy regimens for metastatic disease (liver, skeletal), the patient underwent CT guided needle biopsy of the liver which showed HER2 positive adenocarcinoma (DAKO score $3+$ ). The patient was started on a schedule consisting of vinorelbine $\left(30 \mathrm{mg} / \mathrm{m}^{2} \mathrm{~d} 1,8,15 \mathrm{q} 4 \mathrm{w}\right)$ and trastuzumab (4 mg/kg loading dose, $2 \mathrm{mg} / \mathrm{kg}$ weekly). Results: During a 
treatment period of 4 months severe side-effects were not observed. Imaging results as well as tumor marker kinetics indicate an excellent response with sustained decreasing tumor markers. A retrospective analysis of the HER2 shed antigen in metastatic stage revealed excessively increased serum levels and supports HER2 overexpression observed in liver metastasis. The kinetics of the HER2 shed antigen during therapy for metastatic disease was found to be in phase with the kinetics of CEA and CA15-3. Conclusion: This case report demonstrates that re-evaluation of the HER2 status may be helpful in single patients not sufficiently responding to treatment of metastatic disease. Determination of HER2 overexpression may be facilitated by a determination of the HER2 shed antigen level in peripheral blood.

\section{Poster Session: Genitourinary Cancer \\ P636 \\ Distribution of Histological Subtypes and Molecular Determinants of Treatment Response in Adult Extragonadal Germ Cell Tumours}

Honecker F. ${ }^{1}$, Mayer F. ${ }^{1}$, Looijenga L.H.J. ${ }^{2}$, Oosterhuis J.W. ${ }^{2}$, Bokemeyer C. ${ }^{1}$, Hartmann J.T.

${ }^{1}$ Department of Hematology/Oncology, University of Tübingen, Tübingen, Germany, ${ }^{2}$ Department of Pathology, Josephine Nefkens Institute, Erasmus MC - University Medical Center Rotterdam, Rotterdam, The Netherlands

Purpose: To assess biological and clinical characteristics of extragonada germ cell tumours (EGCT) in view of localization and treatment outcome. Methods: Tumour material of 52 patients (pts) with GCTs was collected from pts treated within two German multicenter trials. Clinically, serum marker elevation with evidence of a retroperitoneal or mediastinal mass is considered specific for the diagnosis without definitive histology. For this analysis, only postpubertal cases with a negative testicular biopsy or unremarkable testicular ultrasonography, full clinical follow-up, and material of sufficient quality and quantity were included, leading to the exclusion of 27 cases. Histological diagnosis was made by a reference pathologist (JWO). Immunohistochemistry (IHC) of proteins involved in apoptosis (bcl-xl, bcl-2 bax), cell cycle regulation (Rb, p21, p53), DNA-mismatch-repair (MLH1, MSH2), nucleotide excision-repair (XPA), and drug export or inactivation (MRP2, BCRP, LRP) was performed using standard methods. FISH analysis was performed using centromer probes for chromosome $\mathrm{X}, 1$ and 12 . Results: A histological diagnosis was possible in 10 retroperitoneal and 15 mediastinal EGCTs. Cure rates were high in pts with retroperitoneal EGCT (80\%), whereas long-term survival was poor in pts with mediastinal EGCT $(27 \%)$. Histologies were as follows: retroperitoneal EGCTs: 2 seminomas (SE), 6 pure choriocarcinomas (CC), and 2 pure yolk sac tumours (YS); mediastinal EGCTs: 1 SE, 3 CC, 5 YS, 2 teratomas, and 4 mixed histologies. In 19 cases, IHC was performed. Compared to a historical series of gonada GCTs, a higher number of cases showed expression of bcl-2, p21, p53, MRP2, BCRP, and LRP. Ploidy assessment using FISH was performed in 17 cases. All tumours with the exception of one diploid mediastinal teratoma were aneuploid, mainly hyperdiploid. No differences between retroperitoneal and mediastinal EGCTs were observed. Conclusions: The observation of a high proportion of pure $\mathrm{CC}$ in the retroperitoneum is unexpected and supports the existence of EGCTs originating in the retroperitoneum without a gonadal primary tumour. IHC results indicate that anti-apoptotic signals, ability to induce cell cycle arrest, or the expression of drug export or inactivation proteins, might confer resistance in individual cases of EGCTs. The existence of a postulated subentity of diploid mediastinal GCTs with a particularly poor outcome could not be confirmed.
P637

\section{Testicular Tumorigenesis Reverses Cell Cycle Gene Expression Patterns of Germ Cell Maturation}

Bäumer N. ${ }^{1}$, Diederichs S. ${ }^{1}$, Sandstede M.-L. ${ }^{1}$, Schrader M. G. ${ }^{2}$ Berkenfeld F. ${ }^{1}$, Möller M. ${ }^{1}$, Schultz N. ${ }^{3}$, Berdel W. E. ${ }^{1}$, Serve H. ${ }^{1}$ Müller-Tidow $\mathrm{C}^{1}$

${ }^{1}$ Department of Medicine, Hematology/Oncology, University of Münster, Germany, ${ }^{2}$ Department of Urology, University Hospital Benjamin Franklin, Berlin, Germany, ${ }^{3} \mathrm{Cecil} \mathrm{H}$. and Ida Green Center for Reproductive Biology Sciences, Department of Pharmacology, University of Texas Southwestern Medical Center Dallas, TX, and Institute of Biochemistry, Free University Berlin, Germany

Testis cancer shows distinct clinical and biological features compared to other solid tumors. Germ cells in the testis switch from proliferative mitosis to the meiotic cell cycle, a process which is reversed in testicular tumorigenesis. The underlying molecular mechanisms of regulation are currently insufficiently characterized.

The aim of our analysis was to derive a global view of the expression pattern of cell cycle regulators at the switch between mitosis and meiosis, especially their reverse regulation in tumorigenesis. Therefore, we first studied expression levels of cyclins, CDKs and CDK inhibitors during postnatal testis maturation by microarray analysis and real-time quantitative RT-PCR. We found induction of cyclins A1, B2, K and M4, CDK2, all CDKL family members, $\mathrm{CDKN} 2 \mathrm{c}, \mathrm{CDKN} 2 \mathrm{~d}$ and INCA1 during testis maturation whereas cyclins A2, B1, D2, G1, G2, CDK1, CDK4 and CDK2AP1 were downregulated.

The induction of cell cycle regulators in this process reflected their importance in the meiotic cell cycle and spermatogenesis whereas the other genes with decreased expression probably played a role in the mitotic cell cycle. We compared these results with expression changes between human testis tumors and normal testis tissue. To first confirm similar regulatory mechanisms between the different species, we analyzed exemplarily the activity of the human and the murine cyclin A1 promoter in testis maturation by generation of a mouse line expressing EGFP and $\beta$-galactosidase under control of the human and the murine cyclin A1 promoter, respectively. Both promoters were activated simultaneously around day 18 of post-natal development. Expression studies in normal and malignant testis samples $(\mathrm{N}=36)$ revealed an inverse cyclin expression pattern than in testis maturation with elevated levels of D-type cyclins and reduced expression of cyclin B2, substantiating the hypothesis that the switch back from meiosis to mitosis during tumorigenesis reverses the expression of cell cycle regulators.

Taken together, we provide a comprehensive expression map of cell cycle regulators at the switch between the mitotic and the meiotic cell cycle and link previously poorly studied proteins to the regulation of meiosis and testicular tumorigenesis.

\section{P638 \\ No Direct Pro-Apoptotic Effect of Immunostimulative DNA (CpG-ODN) on MB-49 Transitional Cell Carcinoma Cells In Vitro}

\author{
Olbert P., Dalpke A., Varga Z., Heeg K., Hofmann R., Hegele A. \\ Universitätsklinik Marburg, Germany
}

Purpose: Th-1 biased immune response is postulated in the mechanism of action of BCG as well as of immunostimulative DNA sequences (CpGODN). Antineoplastic properties of $\mathrm{CpG}-\mathrm{ODN}$ and mycobacterial cell wall-DNA complex could be demonstrated in vivo for different tumor entities. Investigating the mode of action of $\mathrm{CpG}-\mathrm{ODN}$ in $\mathrm{TCC}$, aim of our studies was to evaluate a potential direct pro-apoptotic effect on MB-49 TCC cells in vitro. Methods: MB-49 cell culture is maintained at our laboratory under standardized conditions (DMEM+Glutamax $+10 \% \mathrm{FCS}+1 \% \mathrm{Pen} / \mathrm{Strep}$, $37^{\circ}, 5 \% \mathrm{CO}_{2}$ ). $1 \times 10^{5}$ cells were incubated for 24 hours. Thereafter the following agents were added: 1 . Immunostimulative $1668 \mathrm{CpG}$ (Phosphorothioate (PTO) -backbone); 2. 1668GpC as direct GC control (PTO); 3. Immuno-stimulative Phosphodiester ODN (PO, to exclude immunostimulative PTO-effect); 4. Lipopolysaccharide (LPS); 5. PBS as unstimulated control. After 4 hours of stimulation, quantitative FACS-analysis was performed after Annexin V/Propidium-Jodine staining (Annexin-V-FluosStaining-Kit, Roche ${ }^{\circledast}$, Germany). Additionally, we performed fluorescencemicroscopic examination of the stimulated and stained cells. Results: Compared to unstimulated controls, neither $\mathrm{CpG}, \mathrm{GpC}$, PO or LPS was able 
to increase the rate of apoptotic cells in FACS analysis (see table). This was confirmed by immunofluorescence microscopy.

\begin{tabular}{|l|l|l|}
\hline & Apoptotic cell rate [\%] & Nekrotic cell rate [\%] \\
\hline $1668 \mathrm{CpG}$ & 1,4 & 7,9 \\
\hline $1668 \mathrm{GpC}$ & 1,9 & 11,1 \\
\hline PO & 2,2 & 16,6 \\
\hline LPS & 3,7 & 19,3 \\
\hline PBS & 3,1 & 14,8 \\
\hline
\end{tabular}

Conclusions: Although pro-apoptotic effects of immunostimulatory DNA could be demonstrated in vivo, we could not confirm these results in a TCC cell culture model. We conclude that the pro-apoptotic and potential antineoplastic effect of $\mathrm{CpG}-\mathrm{ODN}$ is mediated immunologically. A direct interaction between CpG-ODN and tumor cell could not be demonstrated.

\section{P639}

\section{The Role of Paclitaxel in the First-Line Treatment of Patients with 'Poor Prognosis' Germ Cell Tumor (GCT) Undergoing Sequential High Dose Chemotherapy}

Hartmann J.T. ${ }^{1}$, Rick O. ${ }^{2}$, Thomas M. ${ }^{3}$, Schleicher J. ${ }^{4}$, Metzner B. ${ }^{5}$, Flasshove M. ${ }^{6}$, Beyer J. ${ }^{7}$, Schmoll H.-J. ${ }^{8}$, Kanz L. ${ }^{1}$, Bokemeyer C. $^{1}$ ${ }^{1}$ Universitätsklinikum Tuebingen, Germany, ${ }^{2}$ Virchow Klinikum Berlin, Germany, ${ }^{3}$ Universitätsklinikum Muenster, ${ }^{4}$ Katharinenhospital Stuttgart, Germany, ${ }^{5}$ Klinikum Oldenburg, Germany, ${ }^{6}$ Universitätsklinikum Essen, Germany, ${ }^{7}$ Universitätsklinikum Marburg, Germany, ${ }^{8}$ Universitätsklinikum Halle, Germany

Purpose: Paclitaxel (T) is an active agent both in cisplatin-refractory and in untreated germ cell tumor (GCT) patients (pts). In order to determine the impact of $\mathrm{T}$ on the survival (OS) and toxicity this analysis compares the results of two prospective trials in pts with 'poor prognosis' GCT. Methods Paclitaxel (T) is an active agent both in cisplatin-refractory and in untreated germ cell tumor (GCT) patients (pts). In order to determine the impact of T on the survival (OS) and toxicity this analysis compares the results of two prospective trials in pts with 'poor prognosis' GCT. Results: 155 pts have been analyzed, 59 pts in HD-VIP and 96 pts in HD-VIP+T. 112 pts and 414 cycles are fully assessable for toxicity, 152 pts for OS. Pts characteristics (HD-VIP compared to HDVIP+T): Median age 29 (16-42) vs. 31 yrs (18-54), mediastinal primary 11 vs. $24 \%$, liver mets 40 vs. $40 \%$, bone 7 vs. $4 \%$, CNS 20 vs. $19 \%$, elevated AFP, HCG, LDH: 39 vs. $30 \%, 61$ vs. $58 \%$ and 77 vs $77 \%$. Dose intensity of the 3-vs. 4-drug regimen achieved have been $98 \%$ and $95 \%$. Median time to recovery of granulocytes and thrombocytes (>500/ $\mu$ l resp. $>25.000 \mu 1)$ have been day 15 and 16 independent from the addition of T. Differences in grade III/IV toxicity in favor of HD-VIP were seen in terms of stomatitis (14 vs. $49 \%$ ), neutropenic fever (3 vs. $20 \%$ ) and manifest infection (3 vs. 19\%) $(\mathrm{P}<.05)$. Grade I/II neurotoxicity was observed in 17 vs. $44 \%$ of pts, and grade III in $0 \%$ vs. $3 \%$ of pts $(\mathrm{P}>.05) .88 \%$ of pts attained a favorable response (NED/CR/PRm-) to HD-VIP compared to $67 \%$ in the HD-VIP+T $(\mathrm{P}<.05)$. After a median follow up period of 34 mos (range, 9-78) and 16 mos (range, 0-47) the calculated 3-yr OS rates were 69.9 (CI95\%, 54.3-83.3) for HD-VIP and 67.3\% (56.3-78.3) for HD-VIP+T $(\mathrm{P}=.3)$. Early death rate due to progression/toxicity was $3 \%$ in both trials. Conclusions: The addition of paclitaxel to HD-VIP dose level 6 as first-line therapy was associated with a moderately elevated toxicity. No substantial impact on survival can be anticipated for HD-VIP+T.

\section{P640}

\section{Antineoplastic Effect of Immunostimulative DNA (CpG-ODN) in a Murine C57-BL6/MB-49 Transitional Cell Carcinoma Model}

Hegele A., Dalpke A., Heeg K., Barth P., Hofmann R., Olbert P. Universitätsklinik Marburg, Germany

Purpose: Intravesical BCG installation is established and efficient in the prophylaxis of recurrent transitional cell carcinoma (TCC), but its mode of action has not yet been elucidated. However, a Th-1 biased immune response is postulated. Cell culture and animal models proved the efficacy of synthetic CpG- oligodeoxynucleotides (ODN) as inducer and adjuvant for a strong
Th1-response and there is evidence for a direct and/or adjuvant antineoplastic effect. Purpose of our studies was to evaluate the antineoplastic effect of locally administered $\mathrm{CpG}$ ODN in an subcutaneous murine bladder cancer model. Methods: A subcutaneous murine TCC model was established with female C57/B16 mice and the corresponding syngeneic MB49 TCC cell line. 3 groups of 5 animals received a cell suspension standardized for $1 \times 10$ cells/50ul. The cells were injected s.c. into the right and left flank. Only phosphorothioate (PTO) - modified ODN were used. Group 1 received 10nmol of CpG-ODN (immunostimulative sequence: $1668 \mathrm{CG}$ ) only into the right cell depot. Group 2 received $10 \mathrm{nmol}$ of $\mathrm{GpC}$ ODN (1668GC) into the rigth cell depot to control for PTO-backbone effects. Group 3 served as untreated control and received only PBS. The animals were examined for tumor size and weight and body weight at various time points after injection until execution on day 14. Tumor or scary tissue at the injection site were excised, weighed and examined histopathologically (HE-stain). Results: Tumor sizes and weights showed no side differences. Average tumor weigth on day 14 was $82 \mathrm{mg}$, $62 \mathrm{mg}$, and $8 \mathrm{mg}$, resp. in groups 3,2 and 1 . Diameter was $8 \mathrm{~mm}$, $5 \mathrm{~mm}$ and $1 \mathrm{~mm}$, resp. There was no measurable weight loss. Histopathology revealed solid vital epithelial tumors in group 3 without a significant inflammatory reaction. The tumors of group 2 showed reduced vital tumor mass, central necrosis and moderate mononuclear infiltration of the surrounding tissue. Group 1 showed almost complete tumor necrosis and a considerable mononuclear inflammatory response. Conclusions: Immunostimulative DNA has promising antineoplastic local and systemic activity in a murine subcutaneous TCC-model. The histological findings suggest an immunologically mediated mode of action. A PTO-backbone effect has to be considered but does not seem to be the decisive element. The results have to be confirmed in an orthotopic TCC model and the immunological response needs further elucidation.

P641

\section{Late relapse in Patients with Germ Cell Tumors (GCT) after Cisplatin-Based Chemotherapy}

Hentrich M. ${ }^{1}$, Gerl A. ${ }^{2,3}$, Weber ${ }^{2}{ }^{2}$, Schlemmer M. ${ }^{3}$, Hartenstein R. ${ }^{1}$, Hiddemann $W^{3}{ }^{3}$

${ }^{1}$ Krankenhaus München-Harlaching, Abteilung Für Hämatologie-Onkologie, München, Germany, ${ }^{2}$ Onkologische Schwerpunktpraxis, München,

Germany, ${ }^{3}$ Klinikum Großhadern der Ludwig-Maximilians-Universität München, Germany

Purpose: Late relapses of germ cell tumors, defined as recurrence after an interval of more than 2 years from initial therapy, are rare with only a few reports published. Methods: We analyzed characteristics, therapy and outcome of patients (pts) with late relapse after cisplatin-based chemotherapy. The records of 37 pts treated at our institutions from 1983 through 2002 were reviewed. Results: 36 pts had a non-seminomatous GCT (pure teratoma in 5 cases), 1 pt a pure seminoma. The median time to late relapse was 66 months (range 25 to 191 months) with 21 pts (57\%) and 5 pts (14\%) relapsing after more than 5 and 10 years, respectively. HCG was elevated in 6 of $36 \mathrm{pts}(17 \%)$, AFP in $21 \mathrm{pts}(58 \%)$. Sites involved were retroperitoneum $(46 \%)$, mediastinum $(24 \%)$, pelvis, retrocrural space, neck, lung and liver (11\% each). 24 pts $(65 \%)$ received chemotherapy (CT) as initial treatment for late relapse, 11 pts $(30 \%)$ underwent surgery and 2 pts refused any treatment. 19 patients $(51 \%)$ achieved no evidence of disease (NED) status after initial therapy for late relapse, 9 of them relapsed again (5 pts with another late relapse). 18 pts (49\%) died of disease and 2 pts are alive with disease. After a median follow-up of 52 months (range 9-168) 17 pts $(46 \%)$ are alive with NED, 10 of them $(27 \%)$ continuously and $7(19 \%)$ after further treatment. 3 of 31 pts $(10 \%)$ who received chemotherapy at some time after diagnosis of late relapse achieved a durable NED status to chemotherapy alone ( 1 patient to oral etoposide after failure of high-dose chemotherapy). 3 of 9 pts $(33 \%)$ who underwent salvage surgery for chemorefractory disease are alive with NED. Conclusions: Disease confined to one anatomic site and lymph node involvement only are predictors of favorable long-term outcome after treatment for late relapse. Surgery plays an important role in the management of late relapse. However, chemotherapy has curative potential even in heavily pretreated patients. After successful treatment of late relapse there remains a substantial risk of second late relapse. All pts with CT-treated GCT should be offered life-long follow-up-evaluations. 
P642

Toll-Like Receptor Expression in MB-49 Transitional Cell Carcinoma Cells in Culture

Olbert P., Dalpke A., Schrader A., Heeg K., Hofmann R., Hegele A. Universitätsklinik Marburg, Germany

Purpose: Receptors of the toll-like family (TLR) seem to play an important role in initiating and mediating innate immune responses. There is hope to define agents that activate selected immune response without causing general toxicity via the TLR pathway. Immunostimulative DNA (CpG-ODN) are able to to trigger antitumor, Th-1 biased immune responses. In vertebrates TLR- 9 detects prokaryotic DNA and synthetic CpG-ODN. To evaluate the potential antineoplastic mechanism of action of CpG-ODN in TCC, we examined the expression of the TLR - family on mRNA-level in a murine TCC cell line (MB-49). Methods:MB-49 cell culture is maintained at our laboratory under standardized conditions (DMEM+Glutamax $+10 \% \mathrm{FCS}+1 \%$ Pen $/ \mathrm{Strep}, 37^{\circ}$, 5\% $\left.\mathrm{CO}_{2}\right)$. Results: MB-49 cells express TLR-2 $\left(\mathrm{rE}_{\mathrm{B}-\mathrm{Ac}} 1 / 2^{\mathrm{ct}}=0,0097\right)$ and TLR-4 $\left(\mathrm{rE}_{\beta-\mathrm{Ac}} 1 / 2^{\mathrm{ct}}=0,00257\right)$. TLR-6 and TLR-9 are not expressed by MB49 cells in culture. Conclusions: From these in vitro data we conclude that the potential antineoplastic effects of $\mathrm{CpG}-\mathrm{ODN}$ in TCC are not mediated by TLR-9 receptors on the tumor cell. The mechanism of CpG-ODN action in vivo seems to be mediated immunologically. These preliminary data need further investigations to detect the mode of $\mathrm{CpG}-\mathrm{ODN}$ action in TCC.

\section{Poster Session: Gynecological Tumors}

\section{P643}

Phase I/II Study of Oral Etoposide Plus GM-CSF as Second-Line Chemotherapy in Platinum Pretreated Patients with Advanced Ovarian Cancer

Baur M. ${ }^{1,2}$, Schernhammer E. ${ }^{1}$, Sevelda $P^{3}{ }^{3}$, Speiser $P^{4}$, Gneist M. ${ }^{1}$, Hudec M. ${ }^{5}$, Dittrich C. ${ }^{1,2}$

${ }^{1}$ Ludwig Boltzmann-Institute for Applied Cancer Research (LBI-ACR Vienna), Vienna, Austria, ${ }^{2} 3^{\text {rd }}$ Medical Department with Oncology, Kaiser Franz Josef-Spital, Vienna, Austria, ${ }^{3}$ Department of Gynecology and Obstetrics, Krankenhaus Lainz, Vienna, Austria, ${ }^{4}$ Department of Gynecology and Obstetrics, University of Vienna, Austria, ${ }^{5}$ Department of Statistics, University of Vienna, Austria

Purpose: The aim of this phase I/II study was to determine the maximum tolerated dose and the dose-limiting toxicities of oral etoposide given days 1 10 followed by a rescue with subcutaneous (s.c.) granulocyte-macrophage colony-stimulating factor (GM-CSF) days 12-19 as second-line chemotherapy in platinum pretreated patients with advanced ovarian carcinoma. Methods: Cohorts of three to six pts were treated with doses of ora etoposide from $750 \mathrm{mg} / \mathrm{m}^{2} /$ cycle escalated to $1250 \mathrm{mg} / \mathrm{m}^{2} /$ cycle, days $1-10$, every 3 weeks. Subcutanous GM-CSF $400 \mu \mathrm{g}$ once daily, days 12-19, was added once dose-limiting granulocytopenia was encountered. In total, 18 pts with a median Karnofsky-index of $80 \%$ (range $70 \%$ to $100 \%$ ), with a median time elapsing since last platinum dose of 10 months (range 1-24 months), with $30 \%$ of all pts showing visceral metastases, were treated at four dose levels (DL) of oral etoposide as follows: DL $1750 \mathrm{mg} / \mathrm{m}^{2} / \mathrm{cyle}$, days $1-10$ without GM-CSF: 3 pts; DL $21000 \mathrm{mg} / \mathrm{m}^{2} / \mathrm{cycle}$, days 1-10, without GMCSF: 3 pts; DL $31000 \mathrm{mg} / \mathrm{m}^{2} /$ cycle, days 1-10, with GM-CSF: 6 pts; DL 4 $1250 \mathrm{mg} / \mathrm{m}^{2} /$ cycle, days 1-10, with GM-CSF: 6 pts. All pts were assessable for toxicity and 16 pts for response. Results: Dose-limiting toxicity was reached at DL 4 with three pts out of six showing WHO grade 4 toxicity. One patient died because of a gramnegative sepsis due to granulocytopenia grade 4 at this DL. The other two pts had granulocytopenia grade 4 at this DL. Thus, the recommended dose was determined to be DL 3 , i.e. oral etoposide $1000 \mathrm{mg} / \mathrm{m}^{2} /$ cycle, days $1-10$, followed by s.c. GM-CSF $400 \mu \mathrm{g}$ days $12-19$. Clinical responses (complete or partial) observed in each patient cohort were at DL 1: $1 / 3$ pts, DL 2: 1/3 pts, DL 3: $3 / 5$ pts, DL 4: $2 / 5$ pts. Conclusions: In conclusion, this phase I/II study defined the maximum tolerated dose and the dose recommended for the therapy with oral etoposide given over 10 days followed by s.c. GM-CSF as second-line chemotherapy in patients with advanced ovarian cancer demonstrating encouraging activity of this regimen which supports further investigation in a phase II study.

\section{P644}

\section{Hyperhomocysteinemia in Patients with Gynaecological Malignancies}

Schroecksnadel K. ${ }^{1}$, Frick B. ${ }^{1}$, Fuith L.C. ${ }^{2}$, Fuchs $D .{ }^{1}$

${ }^{1}$ Institute of Medical Chemistry and Biochemistry, Medical University, Innsbruck, Austria, ${ }^{2}$ Department of Gynecology and Obstetrics,

Hospital Eisenstadt, Austria

Purpose: Hyperhomocysteinemia may develop secondary to B-vitamin deficiency. Recently homocysteine production was observed in tumor cell lines, and homocysteine was proposed as a tumor marker. In various diseases including coronary heart disease but also rheumatoid arthritis or neurodegenerative diseases, a close relationship exists between development of hyperhomocysteinemia and increased concentrations of immune activation markers such as neopterin. Methods: B vitamin status was compared with homocysteine and neopterin concentrations in 20 patients (age: $70 \pm 12$ years) suffering from various gynaecological carcinomas (e.g., $10 \mathrm{Npl}$. ov., $7 \mathrm{Npl}$ corp.). Plasma concentrations of homocysteine and cysteine were measured by HPLC on reversed phase and neopterin concentrations were determined by ELISA (BRAHMS, Berlin, Germany), folate and vitamin B12 by RIA (Chiron, Walpole, USA). Non-parametric Mann-Whitney U-test was used for group-comparisons and Spearman rank correlation analyses were performed Results: Compared to healthy controls, homocysteine and neopterin concentrations were increased in patients (all $\mathrm{p}<0.01$ ), folate concentrations were a the lower end of normal and vitamin $\mathrm{B}_{12}$ concentrations were rather slightly increased. Increased homocysteine only was associated with lower folate concentrations ( $\mathrm{rs}=-0.605, \mathrm{p}<0.01)$ and with higher cysteine concentrations ( $r s=0.463, p<0.05)$. However, there was not any association between homocysteine and neopterin concentrations ( $\mathrm{rs}=0.095$, n.s.). Conclusions: Data demonstrate that hyperhomocysteinemia in patients with malignant diseases is unrelated to immune activation phenomena. This observation is in opposite to what has been observed in several other diseases. Development of hyperhomocysteinemia in the patients with malignant diseases relates to B vitamin disturbances. Increased demand for folic acid is most probably due to tumor cell proliferation rather than to immune activation cascades. However, number of patients is still to small for a definite conclusions.

\section{Poster Session: Sarcoma}

P645

\section{Therapeutic Options in Inoperable Desmoid Tumors - Case Report and Review of the Literature}

Schoeler D., Lindner T., Reichardt $P$

Charité, Campus Virchow-Klinikum, Med. Klinik m.S. Hämatologie/Onkologie, Berlin, Germany

Background: Desmoid tumor, also known as aggressive fibromatosis (AF), is a benign tumor exhibiting fibroblastic proliferation arising from fascial or musculoaponeurotic structures. AF infiltrates locally, but does not metastasize, and tends to recur following local excision. Despite the benign microscopic appearance, and the negligible metastatic potential, the propensity of desmoid tumours for local infiltration is potentially significant in terms of deformity, morbidity and mortality due to pressure effects and obstruction of vital structures and organs. 7-12\% of AF are located at the head and neck. In the general population, desmoids are rare, accounting for $0,03 \%$ of all neoplasms, and have an estimated incidence of 2-4 per million per year. The pathogenesis remains unclear but there may be an association with a local trauma or surgery and exposure to progesterone or estrogen. Case Report: We report the case of a 32 years old woman with an inoperable desmoid tumor in the neck, first diagnosed in November 1997. Radiation therapy was not performed because of the localization near the spine and the risk for a high transverse lesion of spinal cord. In 1998 and 1999 she was treated with non-steroidal anti-inflammatory drugs, toremifen, and later LH-RH agonist. Due to a massive tumour progression, a R2 resection was performed in November 1999. A long-term low-dose chemotherapy with vinblastine and MTX was performed in 2000 without success. Also, an oral chemotherapy with trofosfamide was unsuccessful. In 2002 no therapy was applied and the tumour growth rapidly progressed. In May 2003 the patients neck circumference was $62 \mathrm{~cm}$, and the tumour showed infiltration of spine and lung. Globus sensetion and dyspnea led to a tracheotomy to prevent asphyxiation. From May to July 2003 four cycles of a chemotherapy with doxorubicin and dacar- 
bazine were applied, but did not reduce the tumour mass. Instability of the spine, hypoacusis, anisocoria and a paralysis were signs for progressive disease. The patient died in October 2003. Conclusions: En-bloc resection of the tumour, is the treatment of choice, if possible. Involvement of vital structures can prevent from complete surgical resection. When surgical resection is subtotal, recurrence rates have been reported as high as $70 \%$. Radiation therapy has been used, but a standard procedure is not established yet. Recent reports have indicated that adjuvant therapy with antiestrogens, non-steroida anti-inflammatory drugs, warfarin, vitamin $\mathrm{K}$, and testolacton, either alone or in combination, might be useful. Antisarcoma chemotherapy (doxorubicin and dacarbazine) has shown promising results in small series, as has combination therapy with vinblastine and methotrexate. Further investigation of the molecular genesis of fibroblast mitogenesis might help to develop targeted treatment strategies.

\section{P646}

\section{Familial Retinoblastoma and Leiomyosarcoma: Case Report and Review of the Literature}

\section{Schoeler D. ${ }^{1}$, Lindner $T^{1}{ }^{1}$, von der Schulenburg S. , Pink D. ${ }^{2}$, Reichardt $P$. \\ ${ }^{1}$ Charité Berlin, Campus Virchow-Klinikum, Med. Klinik m.S. \\ Hämatologie/Onkologie, Berlin, Germany, ${ }^{2}$ Charité Berlin, Campus Buch and Helios-Klinikum, Med. Klinik m.S. Hämatologie, Onkologie und Tumorimmunologie, Berlin, Germany}

Background: Retinoblastoma is the most common primary ocular malig nancy of childhood, which results from sporadic or heritable mutations in the retinoblastoma gene, $R B 1$, located at chromosome $13 \mathrm{q} 14$. It is well recognized to occur in two patterns: 1) a sporadic, non-heritable form presenting with unilateral disease and no increase in risk of further malignancies and 2) a genetic, heritable form presenting with uni- or bilateral disease, which is associated with a germ line defect and greatly elevated risk of developing a second malignancy. Case report: We here report a very rare case of familial retinoblastoma and leiomyosarcoma in a father and his daughter.

Father: The 54-year old male was diagnosed with unilateral retinoblastoma of his right eye in 1950 and underwent enucleation in the age of 1, he was not treated by chemotherapy or radiation in the childhood. In 2000 a leiomyosarcoma of his right lower leg with pulmonary metastases was diagnosed. He was treated by multiple chemotherapies including ifosfamide/epirubicin, trofosfamide, doxorubicin/DTIC, and ET-743, radiotherapy and surgery of lung and soft tissue metastases. At the moment the patient is treated by a fifth line chemotherapy with gemcitabine.

Daughter: The 31-year old female was diagnosed with bilateral retinoblastoma in 1973. Both eyes were enucleated in the age of 2 , followed by a radiochemotherapy including vincristine, cyclophosphamide and actinomycin. In 1980 retinoblastoma recurred in the left orbita, she was treated by surgery and radiotherapy. 17 years later in 1997 she was diagnosed with a fronto-basal leiomyosarcoma with local involvement of left orbita. She was treated by radical surgery. Only 4 month later she recurred locally. She underwent a chemotherapy with amifostine, epirubicin and ifosfamide followed by surgery. The second local recurrence of leiomyosarcoma in 2000 was treated by stereotactic radiotherapy. In 2002 she underwent $\mathrm{R} 2$ resection of the $3^{\text {r }}$ local recurrence. Since that time the situation remains stable as documented by MRT. Discussion: The propensity for survivors of heritable retinoblastoma to develop second nonocular malignancies is well known. It was initially reported that second tumours occur within the field of irradiation. This was the case of the here presented daughter. Subsequently, it was demonstrated that second tumours could also develop outside of field of radiation or after chemotherapy, or failed previous radiation or chemotherapy which was the case of the father. The mostly reported second tumor after retinoblastoma is the osteosarcoma, with an incidence of up to $50 \%$; followed by fibrosarcoma (20\%). Leiomyosarcomas have been very rarely reported in soft tissues adjacent to the orbit, femur, and maxilla. Visceral leiomyosarcoma of the bladder in retinoblastoma patients were reported after cyclophosphamide containing chemotherapy. In the presented family also the grandchild is affected by retinoblastoma, fortunately it is under local control by laser therapy. With this familial history systematic screening for tumour symptoms should be performed.
P647

Entstehung von Angiosarkomen nach brusterhaltender Mammakarzinom-Therapie - Initiierung eines Registers

Hartmann J.T. ${ }^{1}$, Hohenberger $P{ }^{2}$

${ }^{1}$ Universitätsklinikum Tuebingen, Germany, ${ }^{2}$ Universitätsklinikum Mannheim, Germany

Hintergrund: In der Nachsorge nach Mammakarzinom, insbesondere nach adjuvanter Bestrahlung, werden immer wieder Angiosarkome nach einer Latenzzeit von 5-10 Jahren beschrieben, bisher meist als einzelne Kasuistiken Eine Inzidenz kann in der BR nicht beziffert werden. Ziele: a) Abschätzung der Inzidenz in der BR, b) Disposition von Patientinnen ? c) Interaktion mit Chemotherapie ?, d) Interaktion mit Strahlenart/-feld/-dosis ? e) Vergleich primärer und sekundärer Angiosarkome bzgl. molekular-biologischer Charakteristika sowie klinisch (Behandlungsmodalitäten, Sequenz, Re-Bestrahlung). Methode: Aufgrund der ungesicherten Datenlage wird der Aufbau eines nationalen Registers zur Erfassung der im deutschsprachigen Raum diagnostizierten Fälle angeregt. Das Register soll mögliche klinische und molekularbiologische Risikofaktoren für das Auftreten von Angiosarkomen bei Mammakarzinomen und die Bedeutung verschiedener prognostischer Faktoren für den Verlauf analysieren. Gleichzeitig soll der Einfluß verschiedener Behandlungsmodalitäten auf den Erkrankungsverlauf untersucht werden. Das Register wird von Seiten der Chirurgie, Strahlentherapie und Internistischer Onkologie in Mannheim und Tübingen geplant. Sollte es möglich sein, aufgrund der im Rahmen dieser Studie erfassten Daten, offene Fragen zum Stellenwert von Operation, Chemotherapie und Strahlentherapie als Behandlungsoption zu klären, kann den betroffenen Patienten ein systematisches Therapiekonzept angeboten werden. Weiterhin soll eine Analyse des Tumormaterials hinsichtlich Charakteristika der Tumorbiologie (Expression multidrug-resistance genes, Proliferationsmarker, Zellzyklusanalyse) erfolgen und mit histologischen Untersuchungen sowie mit primären Angiosarkomen korreliert werden. Durchführung: Die Erfassung von Patienten mit Angiosarkomen erfolgt auf einem einfach gestalteten Fragebogen, der bei der Studienleitung angefordert werden kann. Allen größeren Zentren, die Patienten behandeln, werden Teilnahmebögen aktiv zugeschickt. Auf den genannten Prüfbögen werden alle Patientencharakteristika, sowie die gewählten Therapiestrategien mit minimalen Arbeitsaufwand dokumentiert. Patienten sollen retrospektiv ab dem Zeitraum von 2000 und prospektiv eingeschlossen werden (geplant bis Ende 2007). Literatur: a) Rao J, Dekoven JG, Beatty JD, Jones G. J Am Acad Dermatol. 2003;49(3):532-8. b) Williams EV, Banerjee D, Dallimore N, Monypenny IJ. Eur J Surg Oncol. 1999;25(2):221-2. c) Taghian A, de Vathaire F, Terrier P, Le M, Auquier A, Mouriesse H, Grimaud E, Sarrazin D, Tubiana M. Int J Radiat Oncol Biol Phys. 1991;21(2):361-7.

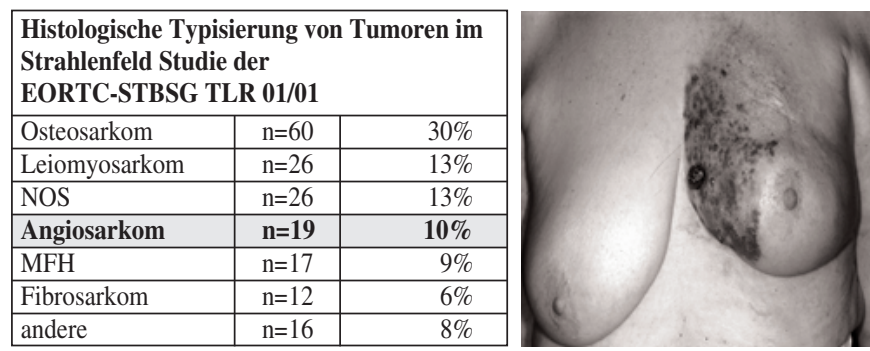

\section{P648 \\ Is there an Indication for High-Dose Chemotherapy in the Treatment of Sarcomas?}

Kasper B. ${ }^{1}$, Lehnert T. ${ }^{2}$, Bernd L. ${ }^{3}$, Ho A.D. ${ }^{1}$, Egerer G. ${ }^{1}$

${ }^{1}$ Department of Internal Medicine V, University of Heidelberg, Germany, ${ }^{2}$ Department of Surgery, University of Heidelberg, Germany,

${ }^{3}$ Department of Orthopaedics, Orthopaedic University Hospital Heidelberg, Germany

Purpose: a question still not answered, as the role of high-dose chemotherapy (HDCT) with autologous peripheral blood stem cell rescue (PBSCT) is not established as standard therapy in the treatment of bone and soft-tissue sarcomas (STS). Patients and Methods: From August 1998 to January 2004 28 patients (pts.) with bone and soft-tissue sarcomas received HDCT and PBSCT (ewing sarcoma family $n=8$, osteosarcoma $n=6$, chondrosarcoma $n$ $=1$, MPNST $n=4$, synovial sarcoma $n=3$, liposarcoma $n=2$, leiomyosarcoma $n=2$, rhabdomyosarcoma $n=1$, meningosarcoma $n=1$ ). 16 pts. were 
female, median age at TPL was 30.4 years [range: 13 - 59]. Following conventional chemotherapy and surgery CR $(n=10), P R(n=10), \operatorname{SD}(n=2)$ and PD $(n=6)$ were reached prior PBSCT. Different conditioning regimens were used, most pts. received the ICE/PEI regimen containing of ifosfamide $2000 \mathrm{mg} / \mathrm{m}^{2} / \mathrm{d} \mathrm{1-6}$, carboplatin $200 \mathrm{mg} / \mathrm{m}^{2} / \mathrm{d} 1-6$ and etoposid $200 \mathrm{mg} / \mathrm{m}^{2} / \mathrm{d} 1$ $6(n=15)$. Results: One pt. died due to cardiac arrest after HDCT. Except hematologic complications, no WHO grade III-IV complications were observed. Four pts. died after PBSCT due to PD, in another 7 pts. disease recurred and led to death, 16 pts. are alive with/without disease. For all pts. median progression-free survival (PFS) is 12.8 months [range: 0 - 61] and overall survival (OS) is 17.6 months [range: $0-61$ ]. Interestingly, osteosarcomas, the ewing sarcoma family and the chondrosarcoma $(n=15)$ demonstrate significant longer PFS and OS with 18.7 months [range: $0-61$ ] and 23.1 months [range: $2-61$ ] compared to STS $(n=13)$ with 6.0 months [0 18] and 11.2 months [range: $0-27$ ], respectively. Of course, that might be due to a significant longer follow-up in the bone sarcoma group with a median of 33.6 months [range: 3 - 68] compared to 23.0 months [range: $5-$ $59]$ in the STS patients, respectively. CR pts. $(n=10)$ showed a median PFS of 25.0 months [range: $3-61$ ] and median OS of 29.6 months [range: $3-61$ ] Conclusion: The remission status prior HDCT seems to be a major prognostic factor for bone and soft-tissue sarcomas, as especially pts. in CR before HDCT could benefit from this treatment strategy.

\section{Poster Session: Solid Tumors}

\section{P649}

\section{Immunocytology in the Diagnosis of Carcinomatous Effusions}

Hastka J., Metzgeroth G., Kuhn C., Schatz M., Hehlmann R. III. Medizinische Universitätsklinik Mannheim, Fakultät für Klinische Medizin der Universität Heidelberg, Germany.

Purpose: Cytological examination is commonly used in clinical practice for clarification of effusions. The method is fast, cheap and needs only a minima apparative equipment. The diagnostic value of cytology, however, is often limited, being considerable depending on the investigators experience. The diagnostic sensitivity and specificity of cytology can considerably be enhanced by immunocytological methods. The immunostaining is performed directly on smears and the result can be evaluated by the conventional light microscope. Methods: Comparing the diagnostic sensitivity of cytology and immunocytology in carcinomatous effusions we investigated samples of 1112 patients. In 551 cases, the effusion was caused by various carcinomas, in 561 cases by other diseases. Results: In carcinomatous effusions, correct diag nosis was done in 292 patients $(53 \%)$ by cytology. In 23 cases $(4,2 \%)$ tumour cells without further specification were described and in 146 cases $(26,5 \%)$ the diagnosis was "effusion suspicious for tumour cells". In 90 cases $(16,3 \%)$ the diagnosis was false negative. In 39 of the 561 nonmalignant samples, the diagnosis was falsely positive.

Using immunocytological staining, correct diagnosis of a carcinomatous effusion was done in 511 patients $(92,7 \%)$ by cytology. In 4 samples $(0,7 \%)$ the diagnosis was "effusion suspicious for tumour cells", the term "tumour cells without further specification" was not used in any of the samples. In 36 cases $(6,5 \%)$ the diagnosis was false negative. Among the 561 nonneoplastic samples, there was no false positive diagnosis. In 201 cases $(36,5 \%)$ of the carcinomatous effusions, the immunocytological staining allowed the localisation of the primary tumour site. Conclusions: Our results show, that immunocytological examination is very sensitive and specific in the diagnosis of carcinomatous effusions. The precise typing of the tumour cells often allows the localisation of the primary tumour site, which minimizes costs by reducing expensive instrumental examination.

\section{P650}

\section{Inhalation Therapy of Lung Metastases with Interleukin-2}

Ulsperger E. ${ }^{1,3}$, Polleres S. ${ }^{1}$, Benold U. ${ }^{1}$, Prasch F. ${ }^{2}$, Geissler K. ${ }^{1,3}$

${ }^{1} 5^{\text {th }}$ Medical Department, Oncology, Hospital Lainz, Vienna,Austria,

${ }^{2}$ Diagnosezentrum Mödling, Austria, ${ }^{3}$ Ludwig Boltzmann Institute for

Clinical Oncology, Lainz, Vienna, Austria

Interleukin-2 (IL-2) treatment has been shown to be effective for several patients with metastatic hypernephroma. This immunotherapy demonstrate in lung metastases response rates of $10 \%$ to $30 \%$ when administered intra- venously or by subcutaneous injections. Severe side effects especially during i.v. administration limit the treatment to patients with good performance status and without comorbidity. Local IL-2 application by inhalation is associated with low toxicity.

122 patients with metastatic renal cell carcinoma were treated in our department with immunotherapy since 1990.24 of these patients received gamma interferon and 74 IL-2 subcutaneously as primary treatment for metastatic disease. 24 patients started IL-2 inhalation for unresectable lung metastases. To demonstrate the distribution of inhaled IL-2 in trachea, bronchial system and lung, we labeled IL-2 with $\mathrm{J}^{123}$. One patient with histological proven lung metastases, responding to IL-2 inhalation previously, consented to inhale radioactive labeled IL-2. We found a homogeneous perfusion of IL-2 in all parts of the lung even in peripheral areas.

In contrast to other publications we use lower doses $-7,2 \times 10^{6}$ I.U. of IL-2 are administered daily for 5 days followed by 2 days rest. Inhalation is performed by the Jetair d20c nebulizer (Hoyer, Germany) on an outpatient basis.

All patients except 2 learned the technique of inhalation with the respirator, while 3 patients stopped using IL-2 inhalation due to cough within the first week. We observed in 19 patients 2 complete-, 5 partial responses and 5 patients with stable diseases. 8 patients progressed. One complete response lasted for 36 month, one patient is still relaps-free after 32 month. None of the patients experienced nausea while cough was usually mild. In this small group of patients we found even with reduced and less toxic dose of IL-2 promising results.

\section{P651}

\section{Non-Invasive Imaging of Subcutaneous Tumors and Pulmonary Metastases in Mice Using Flat-Panel Detector-Based Volumetric Computed Tomography}

\section{Missbach J. ${ }^{1}$, Fork M. ${ }^{1}$, Rieker J. ${ }^{2}$, Missbach $M .{ }^{1}$, Grabbe E. ${ }^{2}$,} Trümper L. ${ }^{1}$, Alves F. ${ }^{1}$

${ }^{1}$ Dept of Hematology and Oncology, ${ }^{2}$ Dept of Diagnostic Radiology, University of Goettingen, Germany

There is a strong need for non-invasive methods to monitor tumor growth and progression over time in order to improve current standard single point data tumor models in mice. This study was aimed at examining the usefulness of flat-panel detector-based cone beam volume-computed tomography (FPDVCT) as a non-invasive technique to monitor subcutaneously growing murine melanomas and their metastatic spread at defined time intervals. C57/BL6 mice at 3 months of age were injected s.c. $\left(1 \times 10^{6}\right.$ cells $)$ or i.v. $\left(1 \times 10^{5}\right.$ cells $)$ with syngeneic B16F1 melanoma cells. Following tumor cell inoculation, scans of anesthetized mice were performed using FPD-VCT (General Electrics Prototype) at distinct intervals. Subcutaneous tumor volumes were also quantitated at the same time points in vivo and post mortem by standard caliper measurement procedure. The results were subsequently related to histological analyses. The experiments validated FPD-VCT of s.c. growing melanomas as a very useful and sensitive technique to reproducibly obtain three dimensional volumetric tumor measurements. The validity and reliability of accurate volumetric calculations of the tumors by FPD-VCT was confirmed by standard caliper measurements of postmortem dissected tumors. Comparative analyses also determined that subcutaneous caliperbased tumor measurements in vivo are highly variable and by far not reliable as FPD-VCT measurements. Furthermore, FPD-VCT was found to be sufficiently sensitive to detect $200 \mu \mathrm{m}$ diameter melanoma micrometastases. However, the dignity of these smallest micrometastases could only be assessed at later stages of tumor progression. Pulmonary tumor nodules of about $500 \mu \mathrm{m}$ in diameter could reproducibly be detected as metastases by FPD-VCT and FPD-VCT measurement of these tumors corresponded well with histological analysis. In conclusion, non-invasive imaging by FPD-VCT allows an accurate real time assessment of subcutaneously growing tumors in longitudinal studies. It is also useful for the earliest detection of pulmonary metastases. Thus, FPD-VCT analysis has the potential to establish a novel standard for real-time non-invasive tumor assessment over time in animal models. 
P652

\section{Ongoing Phase I Study of Pemetrexed Combined with Gemcitabine and Cisplatin in Patients With Locally Advanced or Metastatic Solid Tumors}

Graefe T. ${ }^{1}$, Bolling C. ${ }^{1}$, Lübbing C. ${ }^{1}$, Fleeth J. ${ }^{1}$, Von Scheel J. ${ }^{1}$, Lüdtke F.E. ${ }^{1}$, Müller-Hagen S. ${ }^{2}$, Depenbrock H. ${ }^{3}$, Hanauske A.R.

${ }^{1}$ St. Georg Hospital, Hamburg, Germany, ${ }^{2}$ Oncological Practice, Hamburg, Germany, ${ }^{3}$ Lilly Deutschland $\mathrm{GmbH}$, Bad Homburg, Germany

Purpose: Pemetrexed (P, Alimta $\left.{ }^{\circledR}\right)$ is a novel multitargeted antifolate with clinical activity in a variety of solid tumors, including malignant mesothelioma, lung and breast cancer. Combining P, gemcitabine (G), and cisplatin (C) may lead to synergistic activity. Methods: The aim of this ongoing phase I dose escalation study is to assess the maximum tolerated dose (MTD) and dose-limiting toxicities (DLTs) of different PGC schedules and to collect anecdotal antitumor activity data. To date, the following two schedules have been assessed: Schedule 1 ( $q 3 w$ ): P $400 \mathrm{mg} / \mathrm{m}^{2}$ d1 (escalated to $500 \mathrm{mg} / \mathrm{m}^{2}$ on dose level 2), G $800 \mathrm{mg} / \mathrm{m}^{2} \mathrm{~d} 1+8, \mathrm{C} 40 \mathrm{mg} / \mathrm{m}^{2} \mathrm{~d} 1$. Schedule 2 (q4w): P 500 $\mathrm{mg} / \mathrm{m}^{2}$, G $800 \mathrm{mg} / \mathrm{m}^{2}$, C $40 \mathrm{mg} / \mathrm{m}^{2}$, all on $\mathrm{d} 1+15$. P was administered intravenously (IV) over 10 minutes (min), G IV over $30 \mathrm{~min}$, and C IV over 2 hours. Standard pre- and postmedications were used. Results: On schedule 1 , 12 patients (pts; 9 male, median age 58 years, ECOG Performance Status 0-1) were enrolled, 6 per dose level. Tumor types included head and neck (6), thymoma (1), bile duct (1), prostate (1), renal (1), and cancer of unknown origin (2). A total of 43 courses of Schedule 1 were administered. One pt with head and neck cancer achieved a partial response, and stable disease was observed in 7 pts (for median 10 weeks). 4 of 12 pts experienced DLTs (dose level 1: G3 skin toxicity, febrile neutropenia; dose level 2: G3 diarrhea, G3 syncope). Hematologic toxicities included G3/4 thrombocytopenia in $10 \mathrm{pts}$, G3/4 leukopenia in 8 pts, and G3/4 anemia in 4 pts. Although formal MTD criteria were not met, dose escalation was stopped early in favor of the alternative $\mathrm{q} 4 \mathrm{w}$ schedule. Four patients (all male; median age 47 years, Performance Status 1) were enrolled into the first dose level of schedule 2. Tumor types included head and neck (1), urothelial with a second primary malignancy of pancreatic cancer (1), thymoma (1), leiomyosarcoma (1). Two patients experienced 4 DLTs: G3 fatigue (2), G3 syncope (1), G4 neutropenia requiring delay of study drug on d15 (1). With 7 cycles administered in Schedule 2 to date, there was no G3/4 thrombocytopenia or anemia, 2 of 4 pts had G3/4 neutropenia. G3/4 non-hematological toxicities included G3 fatigue (3) and G3 syncope (1). Conclusions: PGC combination treatment is feasible and has antitumor activity with $1 \mathrm{PR}$ and $7 \mathrm{SD}$. However, the toxicity profiles prompts assessment of alternative $\mathrm{q} 4 \mathrm{w}$ sequential doublet schedule which is currently under investigation.

\section{P653}

\section{Treatment of Advanced Neuroendocrine Tumours with a Combination of Paclitaxel, Carboplatin and Etoposid}

Grothe W., Jordan K., Harba A., Arnold D., Kegel T., Schmoll H.-J. Martin-Luther-University Halle-Wittenberg, Germany

Backround: Combination chemotherapy with paclitaxel, carboplatin an etoposide has been evaluated by Hainsworth et al. (1997) in the treatment of carcinoma of unknown primary. In this study 6 patients (pts.) with undifferentiated neuroendocrine tumours were included. The protocol showed a favorable response and safety profile in comparison to cisplatin/etoposide based regimen which represents the standard treatment for undifferentiated neuroendocrine tumours. Methods: We performed a retrospective analysis of 13 pts. (f/m 7/6 - median age 56 (range 35-75)) with moderate or poorly differentiated neuroendocrine tumours, which have been treated at our center from 1999 to 2003 with paclitaxel $200 \mathrm{mg} / \mathrm{m} 2 \mathrm{~d} 1$, carboplatin AUC $6 \mathrm{~d} 1$ plus etoposide $50 \mathrm{mg}$ alternated with $100 \mathrm{mg}$ orally d $1-10$, qd 22.13 pts. were evaluable for toxicity and 10 for response (3 patients were lost to follow up) Results: A total of 57 cycles were administered (median 4, range 1-14). NCICTC grade III/IV toxicity was anemia $1 / 0$ pt., thrombocytopenia $4 / 1$ pts. leucopenia $2 / 1$ pts., sensory neuropathy $2 / 0$ pts.. In 8 pts. a dose adjustmen due to toxicity was necessary. Efficacy: $3 / 10$ pts. showed a CR, 2/10 pts. a PR and 5/10 pts. a SD. The 2 pts. with a CR were under observation for 18 and 17 mts. with no signs of relapse. Median progression-free survival was 18 month (range 9-47+). Conclusion: The combination of paclitaxel, carboplatin and etoposide is very active in neuroendocrine tumours and is wel tollerated in an outpatient setting. The main toxicity was grade 3 thrombocy- topenia. These promising results justify further evaluation of the combination of paclitaxel, carboplatin and etoposide in a prospective study.

P654

\section{A Randomized Phase II Trial of Gemcitabine Plus Treosulfan Versus Treosulfan Alone in Metastatic Uveal Melanoma}

Schmittel A. ${ }^{1}$, Schmidt-Hieber M. ${ }^{1}$, Bechrakis N.E. ${ }^{2}$,

Foerster M.H. ${ }^{2}$, Thiel E. ${ }^{1}$, Keilholz U.

${ }^{1}$ Charité Universitätsmedizin Berlin Campus Benjamin Franklin, Medizinische Klinik III (Hämatologie, Onkologie und Transfusions medizin), Berlin, Germany, ${ }^{2}$ Charité Universitätsmedizin Berlin Campus Benjamin Franklin, Augenklinik, Berlin, Germany

Purpose: Uveal melanoma is the most common primary malignant tumor of the eye. Metastases occur in 15-40\% of patients and are associated with poor prognosis and a low response rate to chemotherapy. In vitro data suggested synergistic activity of treosulfan and gemcitabine in this disease. Earlier phase I/II trials demonstrated activity of this combination and in addition suggested a survival benefit of patients treated with the maximum tolerated dose (MTD) of the gemcitabine + treosulfan $(\mathrm{GeT})$ protocol when compared to lower dose levels. Methods: We therefore initiated a randomised phase II trial in chemotherapy naïve patients with pathologically confirmed metastatic disease. Exclusion criteria were Karnofsky performance status (KPS) $<60 \%$, symptomatic brain metastases, prior chemotherapy and major organ dysfunction. After stratification by KPS patients were randomly assigned to receive GeT in the MTD of $1000 \mathrm{mg} / \mathrm{m}^{2}$ gemcitabine plus $3500 \mathrm{mg} / \mathrm{m}^{2}$ treosulfan both administered on days 1 and 8 or to $3500 \mathrm{mg} / \mathrm{m}^{2}$ treosulfan alone on days 1 and 8 . Treatment was repeated on day 28. Based on Simon's optimal 2-stage minimax design for phase II trials after 9 patients per arm response rates were analysed in an interim analysis. Results: In the GeT arm 4 patients had a remission or stable disease (PR/SD). In the treosulfan alone arm one patient had a PR/SD. Therefore both arms met criteria for continuation of the protocol until 24 patients per arm will be randomised Conclusions: Final results of this first randomised trial in metastatic uveal melanoma will be presented at the meeting.

\section{P655}

\section{Capecitabine is Active in a Broad Variety of Solid Tumor}

Jakob A., Emmenecker S., Dresel I., Stöckl E., Hirsch F.W.

Department of Hematology and Oncology, Klinikum Offenburg, Germany

Introduction: Capecitabine is an oral fluoropyrimidine carbamate that was rationally designed to be activated preferentially within malignant tumor cells. The final step of activation involves the enzyme thymidine phosphorylase (TP). This enzyme is expressed at significantly higher levels in tumor tissues than corresponding normal cells. Capecitabine has proven to be very effective in metastatic colorectal and breast cancer. A high TP activity has also been shown in a variety of other tumor tissues. In general the side effects are mild and the treatment is well tolerated. Material and Methods: We report on 22 patients with various tumor types who were treated with Capecitabine: 4 patients with pancreatic cancer, 4 patients with lung cancer, 4 patients with head and neck cancer, 2 patients with thyroid cancer, one patient with an uterine cancer, one with an urachus cancer, one with a gastric cancer, one with a choledochus cancer, one with a neuroendocrine tumor of the liver, and one with a carcinoma of unknown primary site. Histologically there were mainly adenocarcinomas and squamous cell carcinomas. Results: Every reported patient experienced at least a disease stabilisation over a 3 months period. Some patients had long lasting remissions with a very good quality of life. The treatment was well tolerated. More details will be presented at the meeting. Conclusion: Capecitabine is established in the treatment of metastatic colorectal and breast cancer. These selected cases of a variety of tumor types demonstrate a broad activity of Capecitabine in cancer treatment. Further clinical trials are necessary to define the role of this compound in the treatment of the different tumor entities. The oral formulation is very suitable for therapy in an out-patient setting. 


\section{Poster Session: Immunohematology}

P656

The Lymphoid Oncogene TCL1 Shows a Stage-Specific Expression Pattern during both T- and B-Cell Maturation

Herling M. ${ }^{1}$, Patel K. ${ }^{1}$, Hoyer K.K. ${ }^{2}$, Teitell M.A. ${ }^{2}$, Jones $D .{ }^{1}$ ${ }^{1}$ Department of Hematopathology, The University of Texas, M. D. Anderson Cancer Center, Houston, TX, USA, ${ }^{2}$ Department of Pathology and Laboratory Medicine, David Geffen School of Medicine at UCLA, Los Angeles, CA, USA

Purpose: Activation of nearly all T-cell oncogenes by chromosomal rearrangements produces immature blastic tumors that are arrested at a particular thymic differentiation stage. An exception occurs in the mature T-cel prolymphocytic leukemias (T-PLL) that develop after insertional activation of the T-cell leukemia-1 (TCL1) gene at the time of T-cell receptor gene rearrangement. Thus, constitutive expression of TCL1 is oncogenic but seems to allow evolution to a mature post-thymic phenotype. To help understanding the function of regulatory as well as aberrantly expressed TCL1, we focus in this report on its expression pattern in the context of lymphocyte differentiation. Methods: TCL1 protein was analyzed by Western blot and immunohistochemistry on normal lymphatic cells/tissue of different maturation stages as well as in various lymphomas/leukemias and cell lines of T- and B-cell lineage. Results: In thymic T-cells, we note that TCL1 is normally expressed in only a small number of thymocytes at the earliest maturation stage (i.e. CD3-CD4-CD8-) and is silenced in later stages of T-cell maturation. Similarly, among precursor T-lymphoblastic tumors $(\mathrm{n}=48)$, TCL1 was expressed only in cases with both an early (stages I and II) thymocyte immunophenotype and an absence of surface CD3 ( $<<0.001)$, except for 1 case. Among post-thymic mature T-cell tumors $(n=214)$, TCL1 was expressed exclusively in T-PLL (44/60; 73\%), the tumor with known TCL1 rearrangements. The level of TCL1 expression in T-PLL was higher than that seen in the TCL1+ cases of stage I/II surface CD3-negative T-ALL/LBL. In normal B-cells, TCL1 was highly expressed in all developmental stages until the germinal center in which expression varied between different stages of follicular maturation. This pattern was replicated in a broad range of primary B-cell tumors and cell lines, of which the follicular lymphomas $(n=60)$ showed the most variable TCL1 expression. Here, the level of TCL1 correlated with markers (i.e. CD10, CD23) reflecting the maturation stage of these germinal center derived tumors. Conclusions: In T- and B-cells and derived lymphoid tumors, TCL1 exhibits a maturation stage-specific pattern of expression, except when insertional activation of the gene occurs in T-PLL. Future work will have to clarify the function of this developmentally regulated gene in processes of lymphocyte differentiation allowing potentia insights in its oncogenic mechanisms associated with constitutive expression.

\section{P657}

\section{Clonal Precursors for both Human CD11c ${ }^{-}$Type I Interferon-Producing and CD11 $c^{+}$Dendritic Cells are Contained in Lymphoid and Myeloid Restricted Hematopoietic Progenitor Populations}

\section{Manz M.G. ${ }^{1,2}$, Jarrossay D. ${ }^{1}$, Chicha L..}

${ }^{1}$ Institute for Research in Biomedicine (IRB), Bellinzona, Switzerland, ${ }^{2}$ Medizinische Klinik II, Tuebingen, Germany

Purpose: Due to different cytokine responsiveness, surface receptor, and transcription factor expression, human CD11c natural interferon-type I producing cells (also called plasmacytoid cells or plasmacytoid pre-dendritic cells) and $\mathrm{CD} 11 \mathrm{c}^{+}$dendritic cells were thought to derive through lymphoid and myeloid hematopoietic developmental pathways, respectively. Methods: To directly test this hypothesis, we established an in vitro assay allowing simultaneous interferon-type I producing cell, dendritic cell, and B cell development (Flt3-ligand supplemented Ac6/Sys-1 stroma cell culture), and tested human lymphoid and myeloid committed hematopoietic progenitor cells isolated from cord blood for their developmental capacity. Results: Lymphoid as well as common myeloid and granulocyte/macrophage progenitors were capable to develop into both functional interferon-type I producing cells and in dendritic cells. Interferon-type I producing cells expressed gene transcripts thought to be associated with lymphoid lineage development irrespective of their lineal origin (e.g. pre-TCR $\alpha$ and Spi-B). Both cell types differentiated within 5-6 cell divisions from input cells as determined by
CFSE dilution. However, myeloid progenitors expanded about two-fold more, and clonal progenitors for both populations were about five fold more frequent within myeloid committed compared to lymphoid committed progenitor cells (about 1 in 18 versus 1 in 92, respectively). Conclusions: CD $11 c^{-}$interferon-type I producing cells, and to some extend also CD $11 \mathrm{c}^{+}$ dendritic cells, are important mediators of innate immunity, and, upon consecutive maturation, initiate antigen specific adaptive immune responses. Innate immunity, however, pre-dates adaptive immunity in evolution. In this view, it is conceivable that both interferon-type I producing cells and dendritic cells in humans as in mice (data not shown) robustly segregate with myeloid development; type I interferon-producing cells then might primarily use genetic programs that later were adopted in adaptive immune system development. It will be important to further characterize developmental checkpoints for both cellular populations. This might lead to identification of potential new therapeutic targets for immunomodulation, e.g. in clinical settings as allogeneic hematopoietic stem cell transplantation.

\section{P658}

\section{Vav-Bcl2 Transgenic Mice Develop Autoimmune Kidney Disease on a Non-Autoimmune Prone C57BL/6 Genetic Background}

\section{Egle A. ${ }^{1}$, Harris A. ${ }^{2}$, Bath ML. ${ }^{2}$, Tarlinton D. ${ }^{2}$, Cory S. ${ }^{2}$ \\ ${ }^{1}$ University Hospital Salzburg, Austria, ${ }^{2}$ The Walter and Eliza Hall Institute, Melbourne, Australia}

Purpose: Autoimmune diseases are multigenic diseases that require a distinct complement of genetic lesions to manifest. In E $\mu$-Bcl2 mice, overexpression of the anti-apoptotic protein $\mathrm{Bcl} 2$ in B-cells alone caused autoimmune kidney disease on a mixed genetic background, but not in the non-autoimmune-prone C57BL/6 strain. This demonstrates that overexpression of Bcl2 in B-cells is not sufficient to cause autoimmune disease. Methods: We expressed $\mathrm{Bcl} 2$ throughout the whole hemopoiesis, using a Vav-promoter driven transgene. Results: A high incidence of autoimmune kidney disease was observed in C57BL/6 Vav-Bcl2 transgenic mice. Compared to C57BL/6 E $\mu-\mathrm{Bcl} 2$ mice, the $\mathrm{Vav}-\mathrm{Bc} 2 \mathrm{mice}$ did not show significantly higher levels of circulating immunoglobulin and glomerula in both types of mice showed an excess of immunoglobulin deposition by immunohistochemistry. Vav-Bc12 mice had an excess of $\mathrm{T}$ cells in spleen and lymph nodes and the mice developed large spontaneous germinal centre reactions. These germinal centres were correlated with the late development of follicular lymphoma in $\mathrm{Vav}-\mathrm{Bcl} 2$ mice (Egle, Blood, 2003). However, mid-aged pre-sick Vav-Bcl2 mice also showed an excess of macrophages in their glomerula. Interestingly, C57BL/6 mice carry a promoter phenotype of the inhibitory Fc-Gamma IIb receptor that renders macrophages less aggressive than in most other strains. In order to dissect the contribution of T-cell help to the development of disease, we crossed Vav-Bcl2 mice with GK5.5 mice, carrying an anti CD4- antibody transgene. This cross effectively depletes CD4 cells from Vav-Bcl2 mice. While these mice did not develop late follicular lymphoma, as predicted, the mice were still able to develop autoimmune kidney disease, albeit with a delay of about 20 weeks (compared to $\mathrm{Vav}-\mathrm{Bcl} 2$ mice). This argues that the contribution of the T-cell dependent immunity to the manifestation of autoimmunity is dispensable and supports our view, that the end-organ damage in the Vav-Bcl2 mice is supported by the enlarged Bcl2-overexpressing macrophage complement. Conclusions: We demonstrate a first model for autoimmune kidney disease on the non-autoimmune-prone C57BL/6 genetic background using a single transgene. While overexpression of Bcl2 in B cells is not sufficient for the development of antibody mediated kidney damage, we present a model where $\mathrm{T}$ cell-independent autoantibody production is sufficient to cause damage to glomerula with a complement of transgenic myeloid cells.

\section{P659 \\ Influence of CD 25- and/or CD69- Depletion on Primary and Secondary Mixed Lymphocytes Reactions}

\section{Gentilini C., Reinhardt A., Niederwieser D.}

Department of Internal Medicine II, University of Leipzig, Germany

Purpose: Donor T-lymphocytes are responsible for severe graft versus host $(\mathrm{GvH})$ reactions after hematopoietic stem cell transplantation. On the other hand they can recognize and kill leukemic cells (graft versus leukemia, GvL). Previous reports showed that selective depletion of alloreactive cells inhibit 
primary mixed leukocytes reactions (MLR), without affecting the reactivity against third party cells. Our aim was to examine the effects of selective depletion of CD25 and/or CD 69 positive cells in primary but also secondary MLRs in order to further characterize the mechanisms that lead to $\mathrm{GvH}$ or GvL effects. Results: Peripheral blood mononuclear cells (PBMC) from normal donors were co-cultured with HLA-mismatched lethally irradiated PBMC. After 24, 48 or 72 hours of culture CD25-, CD69- or double positive cells were depleted using immunomagnetic procedures. On day $5{ }^{3} \mathrm{H}$-thymidine was added and proliferation measured after 16 hours. For secondary MLRs irradiated original stimulator cells or third party cells were added on day 10 of culture and proliferation was measured after 24, 48 and 144 hours. Depletion of CD69 positive cells in the primary MLR $(n=6)$ lead to a suppression of the proliferation to $25 \% \pm 6 \%, 14 \% \pm 3 \%$ and $9 \% \pm 2 \%$ compared to the controls when depletion was performed after 24, 48 and 72 hours of culture, respectively. The removal of CD25 positive cells $(n=5)$ after 1,2 or 3 days of culture resulted in an inhibition to $20 \% \pm 4 \%, 5 \% \pm 1 \%$ and $2 \% \pm 0,3 \%$. Results were not different when both CD25 and CD69 cells were separated at the same time. We then examined the effects of depletion of alloreactive cells on secondary MLRs. Whereas depletion of cells resulted in an ongoing suppression of secondary responses against the original stimulators, only when CD69 and/or CD25 positive cells were separated after 48 or 72 hours of culture reactivity against $3^{\text {rd }}$ party cells was maintained. On day $10+6$ we observed an increase in the proliferation rate against third party to $98 \% \pm 59 \%$ in double depleted cultures. Conclusions: Depletion of alloreactive cells results in an inhibition of primary MLRs whit the best results obtained when depletion is performed 72 hours after begin of the culture and when CD25 alone or both CD25 and CD69 positive cells are depleted. Depletion of alloreactive cells does not affect the reactivity against $3^{\text {rd }}$ party cells in the secondary MLR suggesting that this could be an interesting approach to prevent $\mathrm{GvH}$ reactions without affecting GvL.

P660

\section{Telomerase Activity and Telomere Length in $\mathrm{CD}^{+} \mathrm{CD} 25^{+}$} Regulatory T-Cells after Expansion in Vivo and in Vitro

Wolf D. ${ }^{1}$, Wolf A.M. ${ }^{2}$, Koppelstätter $C^{3}{ }^{3}$, Rumpold $H^{.}{ }^{1}$, Gastl G. ${ }^{1}$, Mayer G. ${ }^{3}$, Gunsilius E. ${ }^{1}$, Tilg $H^{2}{ }^{2}$

${ }^{1}$ Department of Hematology and Oncology, ${ }^{2}$ Department of Hepatology and Gastrenterology, ${ }^{3}$ Department of Nephrology

Internal Medicine, University of Innsbruck, Austria

The expandability of $\mathrm{CD} 4{ }^{+} \mathrm{CD} 25^{+}$regulatory T-cells (Treg) has been convincingly shown in vitro and in vivo. Activation of telomerase activity is a prerequisite for clonal expansion and concomitant telomere maintenance in proliferating T-cells. Our current report corroborates recently published data showing that Treg isolated from healthy volunteers have significantly shortened telomeres when compared to their $\mathrm{CD} 4{ }^{+} \mathrm{CD} 25^{-}$counterparts. This observation has recently led to the hypothesis that Treg are highly differentiated T-cells showing a senescent phenotype. We therefore next focused on telomere length of Treg from cancer patients, which are known to be expanded in vivo. Of note, telomere length is not shortened in Treg isolated from patients with epithelial malignancies, despite their in vivo expansion. In accordance, expanding Treg rapidly induce telomerase function after in vitro activation with anti-CD3 and IL-2. Induction of telomerase activity is likely to compensate further critical telomere loss under conditions of in vivo expansion. In contrast, sorting of in vitro proliferating Treg using dilution of carboxy-fluorescein diacetate succinimidyl ester (CFSE) revealed a significant telomere shortening in Treg with high proliferative capacity. The latter are characterized by high telomerase activity, which however seems to be insufficient to avoid telomere shortening under conditions of strong in vitro stimulation. This observation should be kept in mind when considering the application of extensively in vitro expanded Treg for the treatment of graft versus host disease (GvHD) or autoimmune diseases.
P661

Evaluation of ZAP-70 in Patients with Chronic Lymphatic Leukemia by Flow Cytometry by Routine Analysis in the Clinical Setting

\section{Wilhelm C., Neubauer A., Brendel C}

Department of Hematology, Oncology and Immunolgy, Philipps-University of Marburg, Germany

Former data indicate ZAP-70 as an excellent prognostic parameter in patients with chronic lymhocytic leukaemia (CLL), as ZAP-70 is strongly associated with the mutational status of the immunoglobulin heavy chain gene ( $\mathrm{IgVH})$ This parameter could thus be relevant for the decision for the time and the mode of treatment in patients with B-CLL. Flow-cytometry is a useful tool in determining antigen expression in a quick and easy manner. Directly conjugated antibodies directed against ZAP-70 are now available. We sought to investigate the feasibility and usefulness of this direct immunfluorescent staining and subsequent cytometric analysis because it is a rapid and easy method compared to western blotting, immuncytochemical staining, indirect intracellular immunfluorescence staining or analysis of the $\mathrm{IgVH}$ mutationa status. Methods: Peripheral blood (15)and bone marrow (1) of patients with B-CLL were first stained with PE or TC conjugated surface CD3 (Beckton Dickenson) und PE conjugated surface CD56 (Beckton Dickenson) antibodys and subsequently prepared according to the FIX and Perm protocol (An der Grub) with an additional washing step in the end, stained with the Alexa Fluor conjugated ZAP-70 antibody from Caltag. Samples were analysed by FACScan and cellquest software from Beckton Dickenson. Results: T cells and NK cells were used as reference population for positive staining. 4 of 16 ( $25 \%$ ) samples were positive for ZAP-70 according the cutoff of $20 \%$ for the lymphocytes as published by Crespo et al. (N Engl J Med 2003;348:1764-75). Range of ZAP-70 expression reached from 0,02 until 75,96\%. Conclusion: Routine staining for ZAP-70 by the Caltag ZAP-70 direct conjugated antibody is easy and feasible. Together with flow-cytometric CD38 evaluation it might be a helpful diagnostic tool for evaluation of disease progression.

\section{P662}

\section{A Sensitive and Reliable Method to Detect Cytoplasmic $\mu$ - Chains by Flow Cytometry}

Heinsch M., Haase S., Giagounidis A.A.N., Schroeder M., Aul C. St. Johannes Hospital Duisburg, Medizinische Klinik II, Germany

B-lineage differentiation is normally detected by the use of antibodies agains cCD79a, cCD22, CD19 or c $\mu$-chains. Because only B-cells synthesize Immunoglobulins (Ig), detection of cytoplasmic Ig constitutes the most characteristic B-cell marker at advanced stages of cellular differentiation. Ig are commonly detected by using fluorescent antibody techniques with anti-Ig conjugated with a fluorescent dye. The detection of Ig is accompanied with certain technical problems. Anti Ig sera tend to bind non-specifically to Fcreceptors, and most cells with Fc-receptors passively acquire Ig. Furthermore in some autoimmune diseases the serum contains antilymphocytic antibodies that attach to the cell surface of T-cells by means of the Fab fragment Another critical point is the discrimination between surface Ig and cytoplasmic Ig. Due to this difficulties fluorescence microscopy is the mos commonly used technique for the detection of $\mathrm{c} \mu$-chains. Microscopy allows the simultaneous analysis of the morphology of the cell and the localization of the attached antibodies. We present a simple reproducible method that detects intracytoplasmic or surface $\mu$-chains in a much more sensitive way than with fluorescence microscopy. By the use of multiparametric differentiation studies of normal B-cell precursors from post-chemotherapy bone marrow, we could clearly demonstrate the reliability of our method.

First, free IgM has to be removed from the cell suspension by two washing steps. Afterwards all membrane bound IgM-molecules are blocked with purified unconjugated goat anti- $\mu-\mathrm{F}(\mathrm{ab}) 2$ fragments in the cold. Subsequently the cells are incubated with further antibodies directed against the interesting targets on the cell surface. Then the cells are fixed, washed again and permeabilised. After this, the cells are incubated with purified Fluorescein Isothiocyanate (FITC)-conjugated goat anti- $\mu-F(a b) 2$-fragments of the same clone blocking the surface $\mu$-chains and further antibodies against intracytoplasmic antigens can be added. The cells are washed again and resuspended. As negative control, the use of goat isotype $F(a b) 2$-fragments is indispensable because $\mathrm{F}(\mathrm{ab}) 2$-fragments show complete different binding features compared to complete antibodies. 
In conclusion we present a reliable and sensitive method that enables us to detect cytoplasmic $\mu$-chains by flow cytometry, thus rendering fluorescence microscopy unnecessary.

\section{Poster Session: Transfusion Medicine}

\section{P663}

\section{Dendritic Cells Generated from Acute Myeloid Leukemia (AML) Blasts Maintain the Expression of Leukemia Associated Antigens}

\section{Li L. ${ }^{1}$, Reinhardt $P{ }^{2}$, Schmitt A. ${ }^{2}$, Greiner J., Ringhoffer M. ${ }^{1}$ Döhner $\mathrm{H}^{1}$, Wiesneth $\mathrm{M}^{2}{ }^{2}$, Schmitt $M$. \\ ${ }^{1}$ 3rd Dept. of Internal Medicine, ${ }^{2}$ Dept. of Transfusion Medicine, University Hospital Ulm, Germany}

Purpose: Recently, the focus is on new specific immunotherapies for AML such as cellular therapies employing dendritic cells (DCs) generated from AML blasts. AML-DCs express constitutionally leukemia associated antigens (LAAs) present in AML blasts they are generated from. Here we investigated whether the generation of AML-DCs would alter the expression level of LAAs. Moreover, we evaluated the presence of HLA and costimulatory molecules on AML blasts versus AML-DCs. Materials and Methods: Quantitative real-time PCR was performed for the following LAAs: preferentially expressed antigen in melanoma (PRAME), the receptor for hyaluronic acid mediated motility (RHAMM/CD168), Wilms tumor gene 1 (WT-1) and proteinase 3. The expression of HLA-ABC, HLA-DR, CD40, CD 80, CD83 and CD86 was evaluated by flow cytometry. AML blasts and AML-DCs were evaluated in ELISPOT assays and Cr-51 release assays for the recognition of $\mathrm{T}$ cell epitope peptides derived from PRAME and RHAMM recognized by CD8+ T lymphocytes. Results: Quantitative real-time PCR for AML-DCs versus AML blasts showed an alteration in mRNA expression of LAAs. An elevated PCR signal for PRAME was detected in 7/12 AML-DC preparations. 6/12 AML-DC preparations showed a significant upregulation of the PCR signal for RHAMM. A stronger WT-1 and proteinase 3 signal was observed in PCR for only 2/12 respectively 1/12 AML-DCs. All preparations showed a strong expression of at least one of the LAAs examined. As demonstrated by flow cytometry, AML-DCs strongly upregulated costimulatory molecules like CD40 and CD80 in comparison with AML blasts. CD8+ T cells recognized $\mathrm{T}$ cell epitope peptides derived from PRAME and RHAMM on AML blasts (40-50\% specific lysis) and to a higher extent on AML-DC (100\% specific lysis). Conclusions: AML-DCs might constitute a powerfu tool in immunotherapy for AML. Real-time PCR allows a quick and quantitative assessment of LAA expression with only $10^{5}$ DCs and might be helpful for the decision whether the AML-DC vaccination strategy is favourable or not. These qRT-PCR results were confirmed by $\mathrm{T}$ cell assays.

\section{P664}

The Predictability of the CD34+ Cell Yield Harvested by Two Automated Leukapheresis Programs: Results of a Multicenter Trial

\section{Schwella N. ${ }^{1}$, Movassaghi $K^{2}{ }^{2}$, Schmitt-Thomssen $A .^{3}$,} Fischer E. G. ${ }^{4}$, Jaques G. ${ }^{1}$, Paulus M. ${ }^{2}$, Heuft H.G. ${ }^{3}$, Neubauer $A$. ${ }^{1}$ Dept. of Hematology and Oncology, Philipps University, Marburg, Germany, ${ }^{2}$ Dept. of Transfusion Medicine, Humboldt University, Berlin, Germany, ${ }^{3}$ Dept. of Transfusion Medicine, Medical School, Hannover, Germany, ${ }^{4}$ Aix Scientifics $®$, Aachen, Germany

Purpose: In a prospective multicenter trial we investigated the predictability of the yield of peripheral blood progenitor cells (PBPC) harvested by the leukapheresis programs MNC and RV-PBSC of the Fresenius cell separator COM.TEC $^{\circledR}$. Based on the preleukapheresis CD34+ cell count, the machine calculates the total yield of CD34+ cells collected during the procedure Methods: A total of 166 donors (128 patients with malignant diseases and 38 healthy individuals) underwent 203 leukaphereses either with the MNC or the RV-PBSC program in three medical centers. Results: In total, 112 collections were performed in 91 donors using the MNC program and 91 harvests in 75 donors using RV-PBSC. The median CD34+ cell collection efficiency (CD34-CE) was significantly higher in MNC than in RV-PBSC program $(\mathrm{p}<0.0001): 68 \%(31-205)$ vs. $44 \%(11-100)$. On the other hand, the median preleukapheresis CD34+ cell count was significantly lower in donors under- going the MNC program than in donors harvested by RV-PBSC $(\mathrm{p}=0.0261)$ : $51 / \mu \mathrm{L}$ (6-834) vs. 64/ $\mu \mathrm{L}$ (12-813). Therefore, the difference between both programs concerning the yield of collected CD34+ cells per apheresis was not statistically significant (MNC vs. RV-PBSC): $384 ? 10^{6} \mathrm{CD} 34+$ cells (22$3281)$ vs. $262 ? 10^{6} \mathrm{CD} 34+$ cells $(30-2521)$. The correlation between circulating CD34+ cells and the number of collected CD34+ cells was highly significant for both programs: $r=0.947(\mathrm{p}<0.0001$; MNC) and $\mathrm{r}=0.904$ $(\mathrm{p}<0.0001$; RV-PBSC). However, the software-predicted CD34+ cell yields were in median higher than the really harvested CD34+ cell numbers. The ratio of the collected and predicted yield of CD34+ cells was significantly higher with $85 \%$ (31-176) for MNC than 59\% (22-110) for the RV-PBSC program $(\mathrm{p}<0.0001)$. There was a significant difference between both programs in the concentrate volume $(\mathrm{p}<0.0001$; MNC vs. RV-PBSC): 383 $\mathrm{mL}$ (204-586) vs. $148 \mathrm{~mL}$ (95-250). Concentrates harvested by the RV-PBSC program had a significantly higher percentage of mononuclear cells and a lower red blood cell (RBC) content than those obtained by $\mathrm{MNC}(\mathrm{p}<0.0001$; p<0.0001): $86 \%$ (43-99) vs. $56 \%$ (25-95) and $12.5 \mathrm{~mL}(3.8-47.7)$ vs. $24.9 \mathrm{~mL}$ (4.7-60.5), respectively. Conclusions: The higher CD34-CE and the more exact prediction of the CD34+ cell yield make the MNC program a safe and convenient leukapheresis procedure. However, when a PBPC concentrate with a low RBC content is required (AB0-incompatible allografts), the RVPBSC program should be preferred.

\section{P665 \\ Use of Paper Sheets in a Clean Room Area Category B according to GMP Guidelines: Proof Of Feasibility}

Kopp J., Hildebrandt M., Ludwig W.-D., Dörken B.

Medizinische Klinik m.S. Hämatologie, Onkologie und

Tumorimmunologie, Robert-Rössle-Klinik im HELIOS Klinikum Berlin, Charité Campus Buch, Berlin, Germany

Background: The feasibility of documentation using paper sheets in clean room areas category B according to GMP guidelines has been put into question repeatedly. Concerns addressed the release of particles in unacceptably high numbers. We have developed and validated a procedure for the use of paper sheets which proved to be associated with acceptable numbers of released particles. The procedure involves the exposure of the paper to dry heat at a temperature of $90^{\circ} \mathrm{C}$ for at least 2 hours. Methods: 50 Paper sheets (white, DIN-A4, wood free fibre) were exposed to dry heat at $110^{\circ} \mathrm{C}$ for $4 \mathrm{~h}$, allowing the paper to reach a minimum core temperature of at least $90^{\circ} \mathrm{C}$ for at least two hours. For comparison, another set of 50 paper sheets was tested without heat exposure. The paper sheets were placed on a table in the clean room area. Particle detectors were placed in front of the table and particles were counted during the following procedures: A) room in operation, paper untouched, B) operator in front of particle detector, paper untouched, C) writing on paper using a ball pen, D) moving and turning of the paper sheets, E) vigorous pounding of the paper sheets on the table. Particles were counted per $\mathrm{m}^{3}$, with the air stream moving towards the detector. The tests were performed four times in two different clean room suites. Results: The particle numbers never exceeded the maximum values allowed in clean room areas category B in operation according to GMP guidelines ( $\geq 5 \mu \mathrm{m}: 2000$ per $\mathrm{m}^{3}, \geq$ 0,5 $\mu \mathrm{m}$ : 350000 per $\mathrm{m}^{3}$ ). However, the particle numbers were lower when using heat-exposed paper sheets than in untreated paper (table). Microbiological testing revealed no colony-forming units on the surface of heat-exposed paper. Conclusions: The use of paper sheets in clean room areas category B according to GMP guidelines is feasible. In view of the low particle numbers and the negative results in microbiological assays, the use of heat-exposed paper as described above should be preferred to untreated paper.

\begin{tabular}{|l|l|l|l|l|}
\hline & \multicolumn{1}{|c|}{$\begin{array}{c}\text { writing using a } \\
\text { ball pen } \\
\text { (particles per } \\
\mathrm{m}^{3} \text {, min-max) }\end{array}$} & $\begin{array}{c}\text { moving and } \\
\text { turning of the } \\
\text { paper sheets } \\
\text { (particles per } \mathrm{m}^{3}, \\
\text { min-max) }\end{array}$ & $\begin{array}{c}\text { vigorous pounding of } \\
\text { the paper sheets on the } \\
\text { table } \\
\text { (particles per } \mathrm{m}^{3} \text {, min- } \\
\text { max) }\end{array}$ \\
\hline $\begin{array}{l}\text { Untreated } \\
\text { paper }\end{array}$ & $\geq 0,5 \mu \mathrm{m}$ & $1340-7730$ & $1270-33900$ & $17050-107000$ \\
\cline { 2 - 5 } & $\geq 5 \mu \mathrm{m}$ & $0-35$ & $0-420$ & $455-1715$ \\
\hline $\begin{array}{l}\text { Heat- } \\
\text { exposed } \\
\text { paper }\end{array}$ & $\geq 0,5 \mu \mathrm{m}$ & $635-6200$ & $1600-12000$ & $14100-28420$ \\
\cline { 2 - 5 } & $\geq 5 \mu \mathrm{m}$ & $0-35$ & $0-280$ & $100-420$ \\
\hline
\end{tabular}




\section{Poster Session: New Drugs \\ P666 \\ Development of Hygromas or Severe Edema during Treatment with the Tyrosine Kinase Inhibitor STI571 is not Associated with platelet-Derived Growth Factor Receptor (PDGFR) Gene Polymorphisms}

\author{
Brück P. . , Wassmann B. ${ }^{1}$, Ramos Lopez E. ${ }^{2}$, Hoelzer D. ${ }^{1}$, \\ Ottmann O.G. \\ ${ }^{1}$ Medizinische Klinik III, Department of Hematology and Oncology, \\ Germany, ${ }^{2}$ Medizinische Klinik I, Department of Endocrinology, \\ Johann Wolfgang Goethe-University, Frankfurt, Germany
}

STI571 is active against Bcr/Abl-, cKIT- and PDGFR-driven malignancies Mild to moderate edema is common, whereas severe edema, body cavity effusions and subdural hygromas are rarely observed. These effects have been suggested to involve inhibition of PDGFR signalling, but predisposing factors are unknown. We examined SNPs in the PDGFR alpha and beta gene regions in STI571-treated patients with and without life-threatening edema or cerebral hygromas, and in healthy volunteers. By RFLP analysis of 15 SNPs, the frequencies of genotypes did not differ between the three groups. SNPs of PDGFR genes do not appear to play a role in patient's susceptibility to clinically severe edema formation during treatment with STI571.

\section{P667}

\section{Zoldedronic Acid Modulates Monocyte Function and Differentiation into Dendritic Cells}

Wolf D. ${ }^{1}$, Rumpold H. ${ }^{1}$, Wolf A.M. ${ }^{2}$, Tilg H. ${ }^{2}$, Gastl G. ${ }^{1}$, Gunsilius E. ${ }^{1}$ ${ }^{1}$ Department of Hematology and Oncology, ${ }^{2}$ Department of Gastroenterology and Hepatology, Internal Medicine, University Hospital Innsbruck, Innsbruck, Austria

Bisphosphonates are widely used for the treatment of osteoporosis and metastases of the skeletal system. Recent reports provided evidence that bisphosphonates may not only reduce bone loss but also exert direct anti-tumor and anti-angiogenic effects. In addition, bisphosphonates have been shown to activate $\gamma \delta$ T-cells. Both, the anti-osteolytic as well as the T-cell activating properties depend on their affinity to phagocytosing and/or antigenpresenting cell types, such as osteoclasts and monocytes. The latter represent a major source for Dendritic Cells (DC), which are key players for the regulation of the host's immune response. Hence, it is tempting to speculate that Zoledronic Acid (ZA), a member of the latest bisphosphonates, exert immunomodulatory properties by modulating monocyte/macrophage function as well as their differentiation to DC from monocytic precursor cells. Our current report demonstrates that therapeutical doses of ZA $(0,1$ to $10 \mu \mathrm{M})$ inhibit the generation of DC from CD14+-selected monocytes, as shown by an impaired upregulation of MHC II, CD83, CD86, CD40 and CD54 on DC derived from monocytes pretreated for 24 hours with ZA. This observation is not due to induction of apoptosis of monocytes by ZA. In parallel, ZA also inhibits the LPS-induced activation of NF-kappaB which is a critical factor for DC differentiation. Accordingly, the activation of allogeneic PBMC in a Mixed-Lymphocyte Reaction (MLR) by ZA-DC is signficantly reduced. In addition, ZA dose-dependently inhibits the production of TNF- $\alpha$ by monocytes as well as the phagocytic capacity of macrophages. In contrast, ZA did not exert any effect on isolated T-cells in terms of their phenotype, cytokine profile or their proliferative capacity. Hence, therapeutic doses of ZA alter monocyte/macrophage function and might modulate $\mathrm{T}$-cell activation via direct inhibition of monocyte differentiation into DC.
P668

The Inhibitory Effects of Imatinib on Monocyte Derived Dendritic Cells are not Mediated by PDGF-R and c-Kit

Rupf A. ${ }^{1}$, Appel S. ${ }^{1}$, Weck M.M. ${ }^{1}$, Grünebach F. ${ }^{1}$, Kanz L. ${ }^{1}$, Schoor O. ${ }^{2}$, Brümmendorf T.H. ${ }^{1}$, Weinschenk T. $^{2}$, Brossart $P .{ }^{1}$ ${ }^{1}$ Department of Hematology, Oncology and Immunology, University of Tübingen, Germany, ${ }^{2}$ Institute for Cell Biology, Department of Immunology, Tübingen, Germany

Purpose: Dendritic cells (DC) are the most powerful antigen presenting cells playing a decisive role for the initiation and maintenance of primary immune responses. However, signaling pathways involved in the differentiation of these cells have not been fully determined. Imatinib is a novel tyrosine kinase inhibitor effective against $\mathrm{Abl}$ kinases, c-Kit and platelet-derived growthfactor receptor (PDGF-R) while not affecting other kinases. Methods and Results: Using this compound, we show that human monocyte derived DC generated in the presence of therapeutic concentrations of imatinib show a concentration dependent reduced expression of CD1a, MHC class I and II and co-stimulatory molecules CD40, CD54, and Dectin-1 expression as well as decreased activation-induced secretion of chemokines and cytokines like MCP-1, RANTES, and IL-12. Moreover, exposure to imatinib reduces the capacity of DC to prime $\mathrm{T}$ cell responses that cannot be restored by the addition of IL-12 and is not due to induction of apoptosis or IL-10 secretion. Using Western blot analyses we found that these effects are mediated by a pronounced downregulation of nuclear localized protein levels of NF- $\mathrm{\kappa B}$ family members RelB, RelA and NF- $\mathrm{KB}$ p50 while not affecting the phosphorylation state of p38 MAP kinase and ERK1. To further analyze pathways and molecules affected by imatinib during DC differentiation we performed gene expression profiling utilizing DNA microarrays. These experiments revealed upregulation of lysosomal genes and genes preferentially expressed in monocytes/macrophages. Importantly, utilizing blocking antibodies, tyrosine kinase inhibitors and siRNA experiments we demonstrate that the inhibitory effects of imatinib on DC differentiation are not mediated by PDGF-R and cKit but most likely via c-Abl tyrosine kinase. Conclusions: Our results reveal a novel signaling pathway involved in the development and function of human monocyte derived DC

P669

The Effects of a Combination Treatment with Imatinib and Farnesyltransferase Inhibitors on Chronic Myelogenous Leukemia Cells

Radujkovic A. ${ }^{1}$, Topaly J. ${ }^{2}$, Zeller W.J. ${ }^{1}$, Ho A.D. ${ }^{2}$, Fruehauf S. ${ }^{2}$

${ }^{1}$ German Cancer Research Center, Department E0120, Heidelberg, Germany, ${ }^{2}$ University of Heidelberg, Department of Internal Medicine $\mathrm{V}$, Heidelberg, Germany

Farnesyl protein transferase inhibitors (FTIs) represent a new class of anticancer agents. We were interested whether the farnesyltransferase inhibitors L-744,832 and LB42918 alone and in combination with imatinib (formerly termed STI571) are active in primary CML cells or CML cell lines.

LB42918 was more potent with ED50 $=4 \pm 1 \mu \mathrm{M}($ mean \pm S.D., $\mathrm{n}=3)$ in the BCR-ABL+ cell line EM-3 vs. ED50 = 25 $\pm 2 \mu$ M for L-744,832 after 48 hrs of treatment and assessment by MTT assay. The growth of BCR-ABL+ K562 and LAMA84 cells was not measurably inhibited by L-744,832 doses up to $25 \mu \mathrm{M}$. Similarly, the growth of K562 was not affected by $25 \mu \mathrm{M}$ of LB42918. However, for LB42918 in LAMA84 cells an ED50 value of $30 \pm$ $11 \mu \mathrm{M}$ could be determined. In the FTI-sensitive cell line EM-3, combination index values $(\mathrm{CI})$ obtained using the method of Chou and Talalay indicated synergistic effects following simultaneous treatment with imatinib and FTI $(\mathrm{CI}=0.5 \pm 0.2$ and $0.7 \pm 0.2$ at ED75 for imatinib $+\mathrm{L}-744,832$ and imatinib + LB42918 respectively). Annexin V / propidium showed a strong increase of the apoptotic cell fraction in EM-3 cells treated for 24 hrs by the combination imatinib + L-744,832 as compared to treatment with each drug alone. Growth inhibition of CFU-GM colonies of primary CML cells obtained from 4 patients is stronger after treatment with different concentrations of the combination of both drugs than after monotherapy with either imatinib or L744,832 . In the cell lines K562 and LAMA84 a trend to lower ED50 values of imatinib was determined when up to $5 \mu \mathrm{M}$ of L-744,832 or LB42918 were added, indicating potentiation of imatinib.

On the basis of the observed potentiation effects FTIs may find their place as supplement for CML patients on imatinib treatment. 
P670

The Immunosuppressive Derivative of Rapamycin, RAD (Everolimus), Promotes Growth Arrest in Vitro and has Profound Anti-Tumor Activity in SCID Mouse Models of Hodgkin and Anaplastic Large Cell Lymphoma

\author{
Jundt $F^{1,2}$, Raetzel N. ${ }^{1}$, Kley K. ${ }^{1}$, Mathas $S^{1,2}{ }^{1,}$ Lietz $A .{ }^{2}$, \\ Dörken $B{ }^{1,2}$ \\ 'Department of Hematology and Oncology, Charité, Campus \\ Virchow-Klinikum, University Medicine Berlin, Germany, \\ ${ }^{2}$ Max Delbrück Center for Molecular Medicine, Germany
}

The immunosuppressive macrolide RAD (INN: everolimus), a chemical derivative of rapamycin, has previously been shown to potently inhibit tumor cell growth of Epstein-Barr virus transformed B lymphocytes of posttransplant lymphoproliferative disorders in vitro and in vivo. In this study, we provide evidence that RAD has profound anti-proliferative activity against tumor cell lines of B-cell-derived Hodgkin lymphoma (HL) and morphologically related T-cell-derived anaplastic large cell lymphoma (ALCL), that appear to be resistant to conventional chemotherapy. In addition, we show that RAD blocks cell-cycle progression in G1 phase. Moreover, we established SCID mouse models with xenotransplanted HL and ALCL cells where we measured the inhibitory effect on tumor cell growth in vivo. While daily treatment $(5 \mathrm{mg} / \mathrm{kg})$ with RAD was nontoxic, it markedly suppressed tumor growth, as determined by decreased tumor volume and survival. Our results demonstrate that RAD has potent direct anti-tumor effects in vitro and in vivo on B- and T-cell derived lymphoma cells and might therefore be considered as a new drug used in combination chemotherapy regimens for the treatment of $\mathrm{HL}$ and ALCL.

P671

\section{Selective Telomere Decapping: a Novel Therapeutic} Modality for Leukemia Treatment?

\author{
El-Daly H., Kull M., Zimmermann S., Pantic M., Waller C., \\ Martens U.M. \\ University Hospital Freiburg, Germany
}

Purpose: Telomere maintenance has been shown to be one hallmark of cancer and has been attributed to unlimited growth potential of human cells Telomerase enzyme maintains the telomeres of eukaryotic chromosomes, and is active in most human cancers but, with few exceptions, not in normal human somatic tissues Therefore, telomerase has become an attractive target for development of new cancer therapeutics. Methods: Here we examined the effects of BIBR1532 in different leukemia cell lines as well as in primary cells from patients with AML and CLL. Results: We observed a dose dependent direct cytotoxicity in concentrations ranging from 30 to $80 \mu \mathrm{M}$ Importantly, the in vitro proliferative capacity of normal $\mathrm{CD} 34^{+}$cells from cord blood and leukapheresis samples was not affected by treatment with BIBR1532. Q-FISH analysis revealed that high dose BIBR1532 induced a time-dependent individual telomere decapping which was associated with loss of TRF2 and increased phosphorylation of p53. Conclusions: We conclude that using this class of telomerase inhibitor at higher concentrations exerts a direct cytotoxic effect on malignant cells of the hematopoietic system which appears to derive from direct damage of the structure of individual telomeres and which must be dissected from telomerase suppressed overall telomere shortening.
P673

\section{The Immunomodulatory Drug Leflunomide Inhibits Cell Cycle Progression in B-CLL Cells}

\author{
Decker T., Oelsner M., Weick K., Peschel C. \\ III. Medizinische Klinik, TU München, Germany
}

Purpose: Leflunomide is an immunomodulatory drug, used as a disease modifying antirheumatic agent. The mode of action is debated although pyrimidine biosynthesis at the level of dihydrooroticacid dehydrogenase seems to be the prime target. In addition, inhibition of JAK and Stat phosphorylation as well as inhibition of cell cycle progression has been reported in $\mathrm{B}$ cells. Disease progression in B-chronic lymphocytic leukaemia (B-CLL) is thought to be caused by deregulated apoptosis pathways. However, it is increasingly recognized that a proliferating compartment of cells contributes to the increasing burden of leukemic cells.In our present study, we have analyzed the effect of A77 1726, the active metabolite of Leflunomide, on cell cycle progression in activated B-CLL cells. Methods: viability, cell cycle progression and apoptosis induction was analyzed in purified B-CLL cells using MTT, PI, BRDU and Annexin assays, respectively. Western blot experiments were performed to analyze expression of cell cycle regulatory proteins or protein phosphorylation. Results: MTT tests revealed a dose dependent reduction of viable cells when activated B-CLL cells were cultured in the presence of A77 1726. Annexin/PI (propidium iodide) staining demonstrated that this effect was not due to increased apoptosis. In contrast, cell cycle progression was inhibited at the G1/S transition as confirmed in BRDU staining and PI staining experiments.

Western Blot experiments demonstrated reduced phosphorylation of the Retinoblastoma protein in cells cultured in the presence of leflunomide. In addition, the expression of cyclin A and cyclin D3 was reduced while cyclin D2 and cyclin E expression was not influenced. As a consequence of reduced cyclin A expression cdk2 kinase activity was markedly reduced. Interestingly, the effect of A77 1726 could not be reverted when $100 \mu \mathrm{M}$ Uridine was added to the culture, suggesting that the effect in B-CLL cells was not mediated by inhibition of pyrimidine biosynthesis. We have previously shown that p70s6kinase is involved in cell cycle progression in B-CLL. However, treatment with A77 1726 did not influence p70s6 kinase phosphorylation. In addition, tyrosine phosphorylation was not significantly changed in leflunomide treated B-CLL cells. Although we were able to clearly define the effect of A77 1726 on cell cycle regulation, the "upstream events" remain obscure. Conclusion: Given the favourable toxicity profile of leflunomide as a disease modifying agent in rheumatic disorders, this immunomodulatory drug might represent a new way to inhibit progression of B-CLL. 